
Antiproton and Pion Structure. Functions From Dimuon Production

si

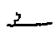

4

$m=$

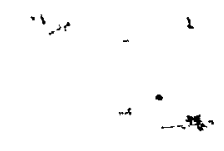

$\vdots$

$+$

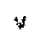

o
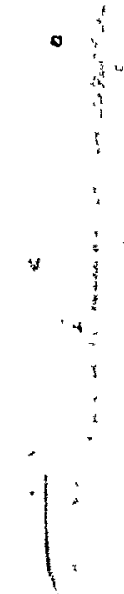

$-4$

$+5$

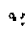

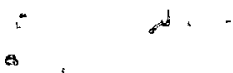

•

$*$

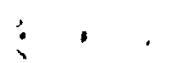

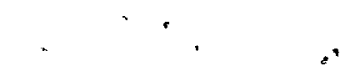
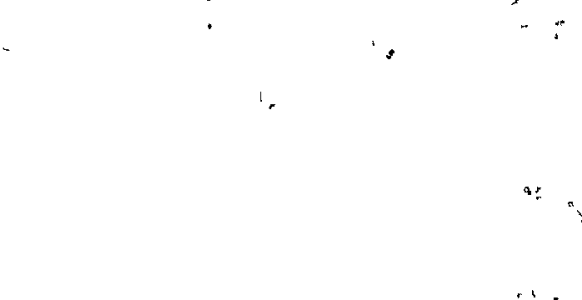

? 



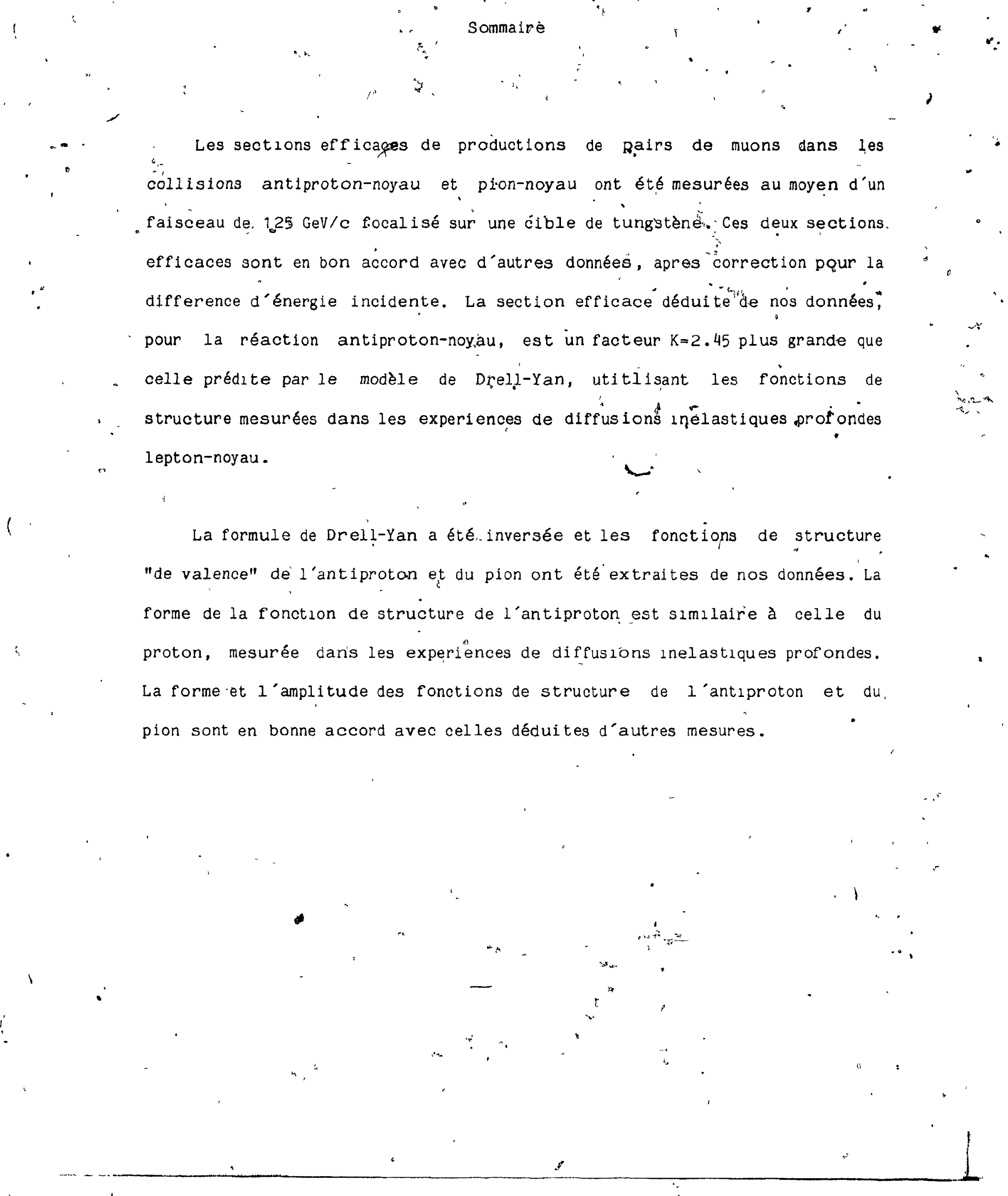




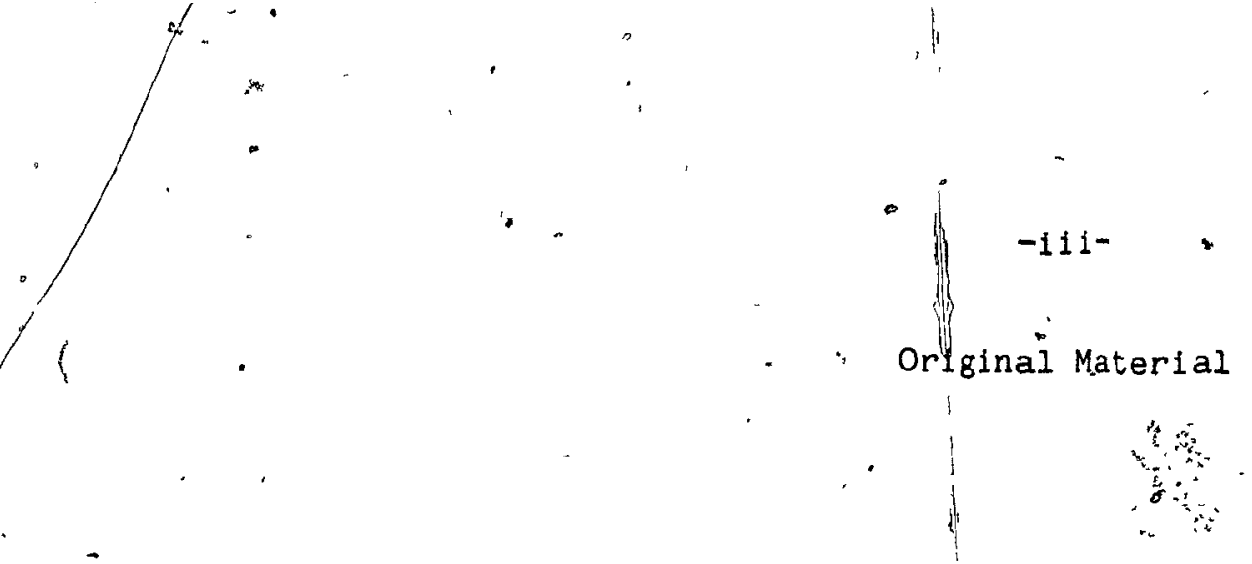

The results obtained by the E537 Callaboration represent the best measurement to date" of muinn pair production in antiproton-nucleon collisions, and aş such, are $a$ good test of the Drell-Yan model. My contributions to the work of the pollaboration included:

1. the testing and installation of one of the drift chambers; :

2. testing and maintenance of the drift chamber electronics system;

3. maintenance of the online software in cooperation with Carl Akerlof, Sergio Conetti, and Dave Nitz;

4. the development of the vertex reconstruction programme in cooperation with Morris Binkley;

5. the development of the Monte Carlo simulation programme;

6. the development of the maximum likelihood fitting programme;

7. the extraction of the stucture functions from the data; and

8. participation in the runging and general operation. of the experiment.

$-1$

称 


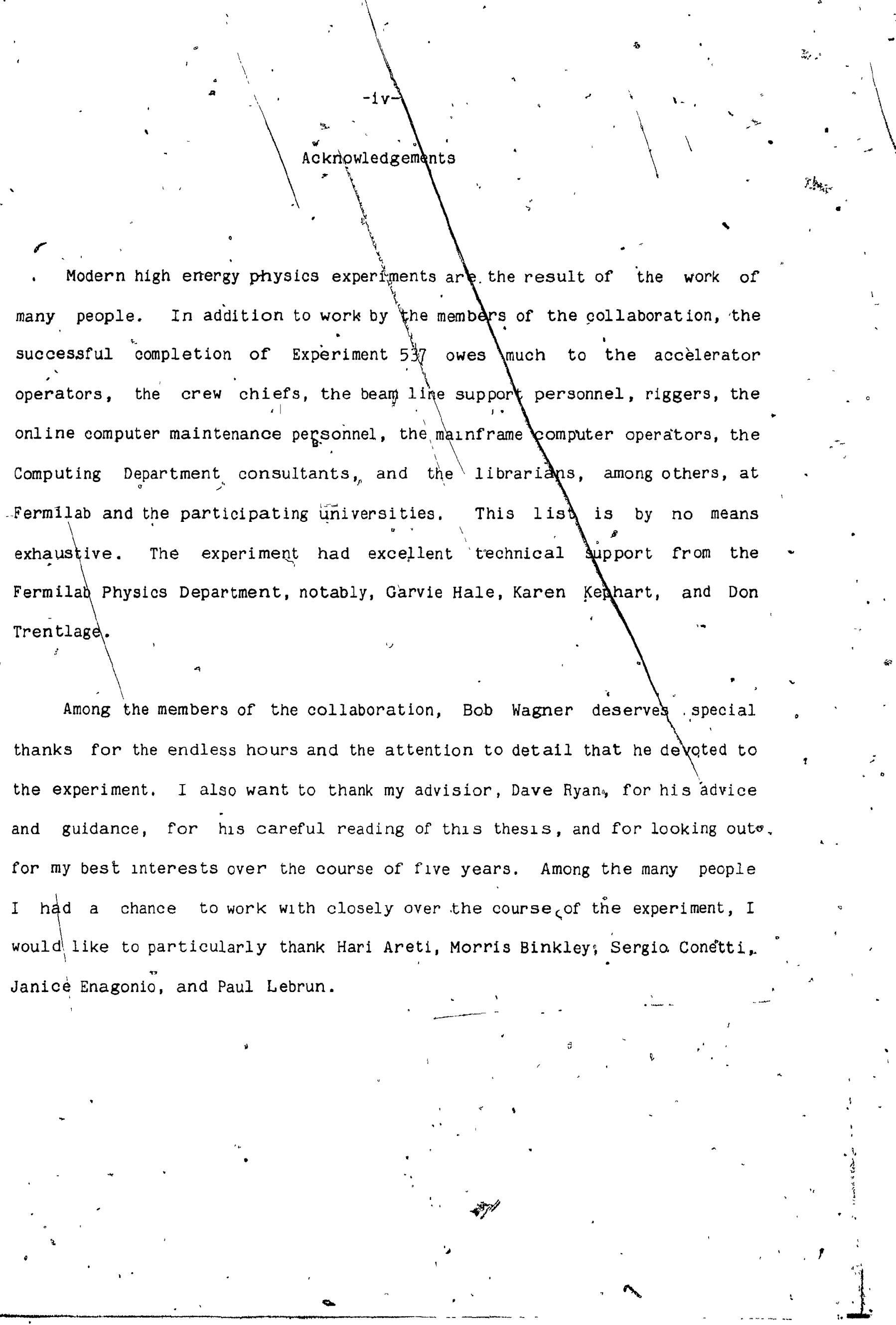




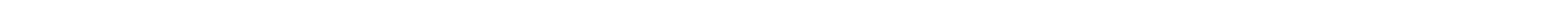


3.8 Target Counters '

3.9 Beam Dump 44

3.10 Absorber Chamber

3.11 Absorber Counter $T$

3.12 Drift Chambers

3.13 Analysis Magnet;

3.14 Gharged Particle Hodoscope

3.15 Muon Hodoscopes

3.16 Fast Logic

3.17 Trigger Processor

3.18 Data Acquisition

3.20 Data Sample

4.0 Reconstruction

4.1 Drift Chamber Calibration

4.2 Drift Chamber Reconstruction

4.3 Beam Chamber Reconstruction

4.4 Vertex and Halo Requirements

4.5 Aperture and Trigger Requirements

4.6 Reconstruction Efficiency

4.7. Kinematic Reconstruction and Data Sample

5.0 Monte Carlo

5.1 Beam Particle 100

5.2 Target Particle.

5.3 Event Generation

5.4 Target and Dump

5.5 Spectrometer 
L

-vil:

5.7 Trigger Processor

5.8 Reconstruction

5.9 Reweighting Events

5.10 Acceptance

6.0 Analysis

6.1 .FItting and Acceptance

6.2 Reconstruction, Counter, and Trigger Efflciencies

$3-$

6.3 Resonance Contamination and Reinteraction

6.4 Beam Flux

6.5 Cross Sections

1

7.0 Results

7.1 Total Cross Sections

7.2 Kinematic Distributions

7.3 Comparisons $W_{1}$ th Other Experiments

7.4 Structure Functions

7.5 Structure Function Projections

7.6 Conclusions

Appendix I - $E 537$ Collaboration

Appendix II - Vertex Reconstruction.

Appendix III - Reinteraction Correction

䧄 


\section{$\because$ List of Figures.}

1 Deep Inelastic Scattering

2 Muon Pair Production

3 Angular Variables

4 QCD Annihilation and Compton Processes

5 Plan View of the E537 spectrometer.

6 High Intersity Area Beam Linë

7 Beam Tagging System

8 CS1 Cerenkov Counter Pressure Curve

9 Beam Log $1 \mathrm{c}$

10 Veto Counters

11 . Veto Counter Loglc

12 Absorber'Counter

13 Charged Particle Hodoscope

14 Charged Particle Hodoscope Logic.

15 Muon Triple Coincidence Counters

16 Muon Triple Coincidence Logic

17 Trigger Logic

18 Trigger Processor Performance.

19 Trigger Processor Efflciency

20 Time to Distance Conversion

21 Drift Chamber Triplets

75.

22 Momentum Calculation

23 Beam Chamber Triplets

24 Beam Momentum Spectrum and Profile

25 Reconstructed Vertex

26 Track Finding Efficiency vs Mass and $x_{F}$

27 Track Finding Efficiency $\mathrm{Vs}_{\mathrm{T}} \mathrm{p}^{2}$ and $\cos \theta$ 
28 Uncorrectéd Antiproton Produced Mass Spectrum 97

29 Uncorrected Pion Produced Mass Spectrum 98

30 Energy Loss for Muons 106

31 Acceptance vs Mass and $x_{F}$ for Antiprotons 111

32 Acceptance vs $\mathrm{p}_{\mathrm{T}}{ }^{2}, \cos \theta$ and $\phi$ for Antiprotons 112

33 Acceptance vs Mass and $x_{F}$ for Pions . 114

$34 \cdot$ Acceptance vs $\mathrm{p}_{\mathrm{T}}{ }^{2}, \cos \theta$ and $\phi$ for Pions 115

35 Antiproton Mass Distribution 126

36 Pion Mass Distribution $\quad 127$

37 Antiproton $x_{F}$ Distribution 128

38 Pion $x_{F}$ Distribution $\quad-\quad 129$

39 Error Ellipsoid. ‘ . 131

$40^{\circ} \psi$ Cross Section vs Target Length $\quad 137$

41 Antiproton Mass Distribution Compared To Drell-Yan Prediction 151

42 Pion Mass Spectrum Compared To Dreli-Yan Prediction 153

43 Antiproton $x_{F}$ Distribution Compared To Drell-Yan Prediotion. 154

44 Pion $x_{F}$ Distribution Compared To Drell-Yan Prediction -155

45 Antiproton $\mathrm{p}_{\mathrm{T}}{ }^{2}$ Distribution . 156

46. Pion $\mathrm{p}_{\mathrm{T}}^{2}$ Distribution 158

47 Antiproton $\cos \theta$ Distribution In The Gottfreid-Jackson Frame 159

48 Pion $\cos \theta$ Distribution "In The Gottfreid-Jackson Frame " 160

49 Antiproton $\phi$ Distribution In The Gottfreid-Jackson Frame 161

50 Pion $\phi$. Distribution In The Gottfreid-Jackson Frame 162

51 Antiproton Scaling Cross Section Compared,With NA3 164

52 Pion Scaling Cross Section Comparison 165

53 Pion Mass Cross Section Comparison 166

54 Pion $x_{F}$ Distribution Compared With CIP . $168=-$

55 . Distribution of Antiproton Produced Events In The $x_{1}-x_{2}$ Plane 769

$56^{5}$ Distribution of Pion Produced Events In The $x_{1}-x_{2}$ Plane. $\quad 170$ 
$-x-$

(. 57 Antiproton Structure Function Fits

58 Pion Structure Function Fits

59 Antiproton Beam Structure Function Projection

60 Antiproton Target Structure Function Projection

61 Antiproton. Beam Structure Function Compared To NA 3

62. Pion Beam Structure Function Projection

63 Pion Target Structure Function Projection

64 Pion Beam Structure Function Comparison

65 Pion Target Structure Function Comparison

66 Multiple Scattering

67 Vertex Reconstruction $7^{-\infty}$

176

177

181

182

183

.185

186

187

188

194

197 


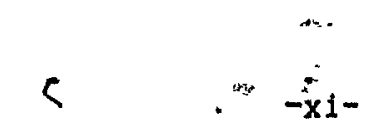

1 List of Tables

1 Quark Quantum Numbers

10

2 Target Densities and Lengths

3 Drift Chamber Parameters I

4 Drift Chamber Parameters II

$2 \times$

5 Fiducial Regions

49

6 Breakdown of Events by Target Configuration

87

$96^{2 .}$

7 . Kinematic Distribution Parameters I

8 Kinematic Distribution Parameters II

9 Reinteraction Correction

10 Incident Beam Flux by Target Conflguration

11 Systematic Error's

144

12 Leading Order Corrections

148

13 Structure Function Parameters 
The production of pairs of muons in the collislons of sub-atomio particles has been extensively studied.since the original observation of a significant signal at Brookhaven in $1970[1]$. This process provides us $^{\text {sith }}$ with a new tool to study the fundamental constituents of nature and their interactions.

Historloally the bulk of our knowledge about the fundamental structure of matter has come from the scattering of elementary particles or the study of the bound states that they form. The scattering of a particles from gold foils in the early 1900's led Rutherford, Gelger and Marsden[2] to propose a picture of the atom as composed of a heavy charged nucleus surrounded by a cloud of orbiting electrons. Problems associated with the clasisical description of the hydrogen atom provided much of the impetus for the subsequent development of quantum mechanics. The discovery of the neutron 
in 1932 by Chadwick[3][4] alłowed all known matter to be described as bound states of the three fundamental particles known at that time, the electron, the proton and the neutron.

*isth the development of the accelerator, it became apparent that sufficiently energetic collisions were able to create more new particles and antiparticles in inelastic collisions. By the end of the 1950 's hundreds of previously unknown particles had been dtscovered, calling into question the very "concept of a fundamental particle. At the same time elastic scattering experiments at Stanford[5] using high energy electrons demonstrated that the proton and the neutron were not point-like particles, but had a finite size, leading to speculation that they themselves might be composite. The proliferating numbers of sub-atomic parbcles took on a pattern if it was assumed that they were, in fact, bound states of fractionally charged constituents named quarks. The quark model, as proposed independently by Gell-Mann[6] and Zweig[7] in 1964, did not identify the quarks with physical objects but rather used them more as a bookeeping device. The experimental state of the art at the time, while able to show that the nucleon was not a point-like object, was not able distinguish any substructure within. Subsequent inelastic electron scattering experiments at Stanford Linear Accelerator (SLAC) in the late 1960's and early 1970's were able to resolye internal substructure. The pattern of scattered"electrons was consistent with a picture in which the nucleon was a bound state of fractionally charged point-like constituents, which were called partons.

The picture that emerged is shown schematically in the Feynman diagrams of Figure 1. The diagrams provide a graphical representation of the mathematical description of the interactions of charged particles in 


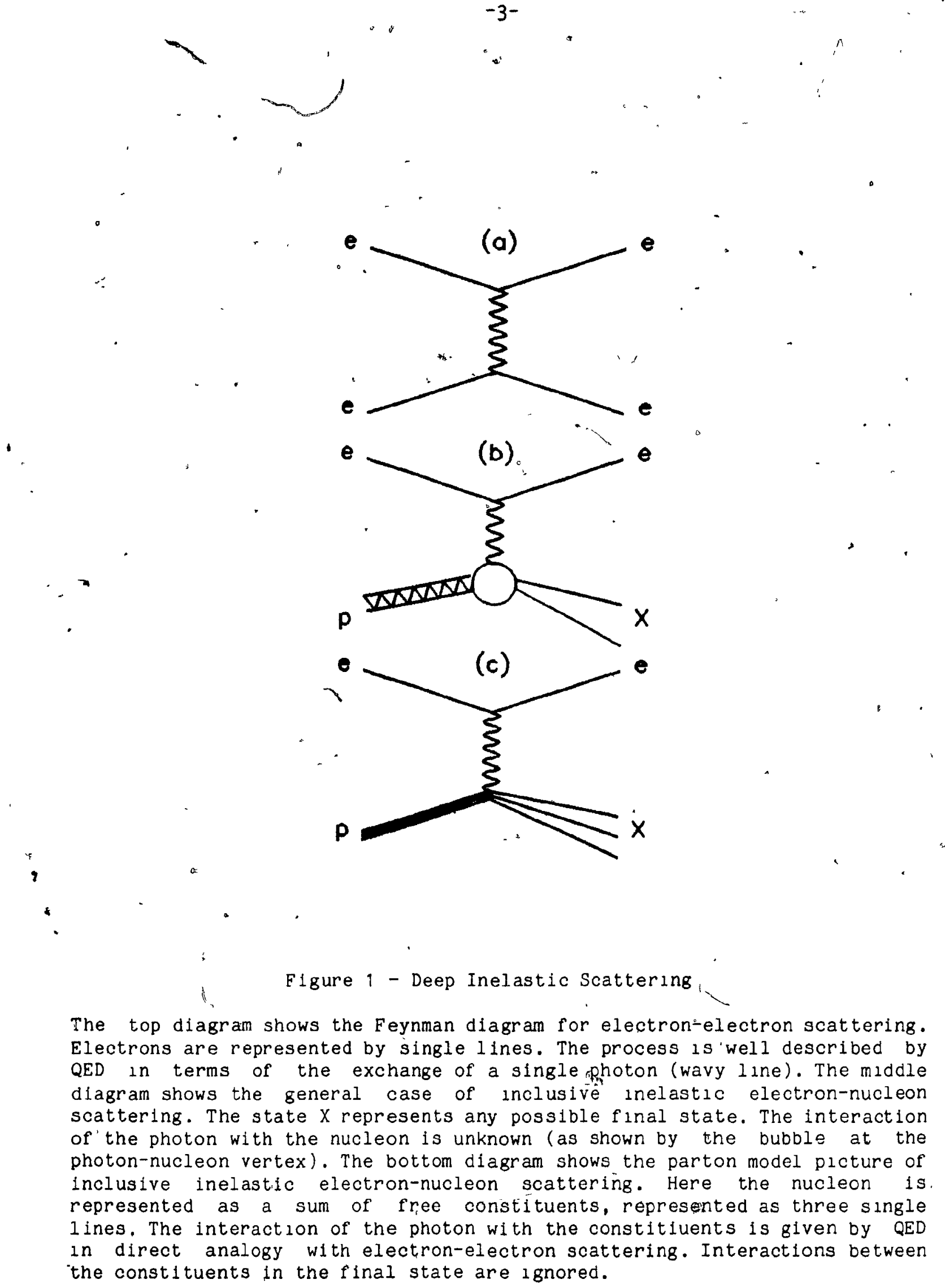




\section{- \\ >}

space-time. The horizontal direction represents the time dimension, while the vertical direction represents, the three spatial dimensions. Figure 1a shows the description of the scattering of two electrons using Quantum Electrodynamics or QED. The point-like electrons are described as quantum mechanical waves and are. represented by single lines. The interaction between the electrons is described by the exchange of a photon, as represented by the wavy line. Figure $1 \mathrm{~b}$ shows the general case of electron-proton scattering. Because the electron cannot interact strongly, the electron part of the interaction is given completely by QED in terms of a point interaction with a photon. The proton, on the other hand, is not a point-like particle, and. its interaction, with the photon, shown as a bubble at the vertex in Figure $1 \mathrm{~b}$, is not known. In the picture of the proton that emerged from the SLAAC results, the oprotion was represented by a sum of fractionally charged point-like constituents represented by the single lines of Figure 10. The interaction of the photon with the proton is now given by the sum of all possible interactions of the photon with the individual constituents, in direct analogy with the QED description of electron-electron scattering. The identification of the constituents with the quarks which had proved so sućcessful in the classification of - sub-atomic resonances paved the way for the emergence of what many feel to be the first viable theory of strong interactions, Quantum Chromodynamics or QCD $[8][9]$

It was "against this background that a columbia-stolny, Brook collaboration at Brookhaven[1], while searching for the w boson in proton-uranium collisions, noticed an anamalously high background of oppositely charged muon pairs. As in the case of deep inelastic electron scattering this phenomenon found an explanation in terms of the partón क 

model, as, shown in the Eeynman diagrams of Figure 2. Figure 2a represents the annihilation of an electron-positron pair to create a pair of muons. As before, the polnt-like electrons and muons are described by quantum mechanical waves; represented by single lines in the diagram, and the interaction is well described if $1 \mathrm{t}$ is assumed that it is mediated by a single photon. The general case of the interaction of a proton and a nucleon, with the associated production of a pair of muons is shown in." F1gure $2 b$. The unknown interaction between the proton and a nucleon is shown as a bubble at the proton-nucleon vertex. Because muons do not interact strongly, the "process must be dominantly electromagnetic, as shown by the photon leaving the bubble and subseqently decaying to give two muons. In the parton model description of this process, as shown in Figure 2c, an analogy is drawn with the QED description of electron-positron annihilation, in the same way that an analogy was drawn between electron-electron scattering and inelastic electron-nucleon scattering. A constituent of the proton annihilates with a constituent of the nucleon to create an intermediate photon which subsequently decays into two muons. If the motion of the constituents inside the hadron is known, either from the solution of bound state wave equations, or from experimental'measurements as in deep inelastic scattering, then the parton model glives explicit predictions for both the magnitude and shape of the spectrum of muon pairs. If the structure of the hadron is not known, as in the case of the pion, the $*$ : inversion of the parton model expression for the oross section allows the motion of the constituents to be determined from the measured distributions

This thesis presents the results of a measurment of the production of muon pairs'in the collisions of $125 \mathrm{GeV} / \mathrm{c}$ antiprotons and pions with a 
tungsten target. Antiproton-nucleon muon pair produation is the best channel in which to compare experiment and theory, since this process is dominated by the annihilation of the constituents whose behaviour is /most easily méasured in inelastic scattering. The difficulties associated with producing a beam of antiprotons of sufficient intensity have inhibited, the study of this process to date. A previous experiment at CERN[10] was aple. to achieve a comparable sample of muon pairs using a $150 \mathrm{GeV} / \mathrm{c}$ pion beam with a small antiproton component $(=2$,percent $)$. The high rates necessary to obtain this sample resulted in a large $('=25$ percent $)$ contamination of pion produced pairs, giving large systematic errors. Many experiments, however, have examined muon pairs produced by pions. The comparison of our pion cross sections with those of other experiments at different energles provides both a test of the scaling predictions of the parton model, and a valuable cross-check on the antiproton results.

The parton model and some of its implications for this experiment are discussed in the next chapter. The corrections required by perturbative QCD are also discussed. Subsequent fhapters discuss the beam and the apparats, the reconstruction of the kinematies of the muon pairs, the computer simulation of the apparatus, and the extraction of the cross sections and kinematic distributions. The final chapter presents our results and kinematio distributions and compares them to the parton model, leading order QCD calculations, and other experiments; The parton model is also used to " extract the structure functions of the antiproton and the pion from the data. 



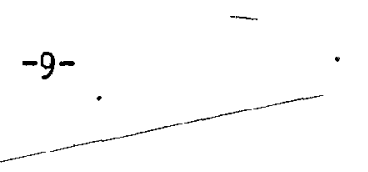

referred to as the Dreil-Yan model, the cross section is given by the sum of: the cross sections for electromagnetic constituent-constituent annihilation into a pair of muons welghted by the product of the relevant structure functions of the respective-hadrons. The structure functions are taken to be characteristic of the hadron rather than the process, so that the structure functions measured in inelastic scattering can be used to predict the cross section for muon pair production.

The parton model is of ten considered to be a zeroth order approximation to the underlying theory of strong interactions, currently belleved to be $=$ Quantum Chromodynamics (QCD). In QCD the hadrons are bound states of fractionally charged fermions called quarks. A list of the quarks and the conventionål assignment[13] of quark quantum numbers is given in Table 1. The quarks are also characterized by a quantum number conventionally called colour. Interactions between the quarks are mediated by field quanta called gluons which also carpy colour. The quarks combine to form bound states in such a way that the net colour quantum number of any observable hadron is zero. The proton is a bound state of two $u$ quarks and a d quark. "The antiprotion is made of two $\bar{u}$ quarks and a $\bar{d}$ quark. "The neutron is a bound state of two d quarks and a $u$ quark. The $\pi^{-}$is a bound state of a $\bar{u}$ and a $d$ quark.

"The bound state equations of QCD should give a complete description of the 'motion of the constituents within the hadrons, but a solution of them has thus far proved elusive. First order pertubative calculations have shown that although the size of the corrections to the parton model plcture are large, the cross section for muon pair production can still be factored into products of process independent structure functions[14]. Questions 
Table 1 - Quark Quantum Numbers

\begin{tabular}{|l|r|r|r|r|r|r|} 
Buryon Number & $1 / 3$ & $1 / 3$ & $1 / 3$ & $1 / 3$ & $1 / 3$ & $1 / 3$ \\
Charge & $-1 / 3$ & $+2 / 3$ & $-1 / 3$ & $+2 / 3$ & $-1 / 3$ & $+2 / 3$ \\
Isospin 2 & $-1 / 2$ & $+1 / 2$ & 0 & 0 & 0 & 0 \\
Strangeness & 0 & 0 & -1 & 0 & 0 & 0 \\
Charm & 0 & 0 & 0 & +1 & 0 & 0 \\
Bottom & 0 & 0 & 0 & 0 & +1 & 0 \\
Top & 0 & 0 & 0 & 0 & 0 & 0 \\
\hline
\end{tabular}

2

;

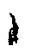

\% 
were ralsed as to whether proofs of factorization could be extended to all orders of perturbation theory [15] but further work[16][17] has ferified that the cross section factorizes up to second order (two loop level).

The production of muon pairs and the Drell-Yan model have been reviewed extensively in the last several years. Lyons[18] and Stroynowski[19] review the production of both low mass and high mass muon and electron pairs, including the $\mathrm{ps}, \psi$, and upsilon, $\mathrm{T}$, resonances whlch can decay via this channel. Kenyon[20] speciflcally reviews the state of the Drell-Yan model. In addition, conference proceedings[21] and workshops[22] provide summaries of much of the recent progress in the field.

2.1 Drell-Yan Model

8

In the parton model picture, the cross section for the production of muon or electron pairs in hadron-hadron collisions is predicted absolutely once the 'structure functions of the hadrons are known from deep inelastic scattering.

The electromagnetic choss sectipn

and a positron into a pair of muons,

$$
\mathrm{e}^{+}+\mathrm{e}^{-}+\mu^{+}+\mu^{-},
$$

is given in the extreme relativistic limit using QED as

$$
\frac{\mathrm{do}}{\mathrm{d} \Omega}=\frac{\alpha^{2}}{4 \mathrm{~s}}\left[1+\cos ^{2} \theta\right]
$$

where

a is the electromagnetic coupling constant,

$s=\left(p_{a}+p_{b}\right) r^{2}$ is the square of the centre of mass energy with $p_{a}$ and $p_{b}$. $\therefore \therefore d$ being the four momenta of the electron and positron respectively, 
$=$ and

$\theta^{3+}$ is the angle between the $e^{+}-e^{-}$axis and the $\mu^{+}-\mu^{-}$axis in the. centre of mass frame.

Integrating over $\theta$, the total cross section for production of a pair of sid

muons is

$$
0=\frac{4 \pi a^{2}}{3 s} \text {. }
$$

Extending these considerations to the case of hadron-hadron collisions, the cross section for a point-like spin $1 / 2$ parton of charge $e_{i}$ with a fraction $x_{1}$ of the momentum $p_{a}$ of one hadron to annihilate with a corresponding antiparton of momentum $\mathrm{x}_{2} \mathrm{p}_{\mathrm{b}}$ in the other hadron and create a pair of muons with momenta $p_{1}$ and $p_{2}$ and an invariant mass of

$$
M^{2}=\left(p_{1}+p_{2}\right)^{2}=\left(x_{1} \dot{p}_{a}^{*}+x_{2} p_{b}\right)^{2}=x_{1} x_{2} s
$$

is

where

$$
d^{2} \sigma=\frac{4 \pi \alpha^{2}}{3 M^{2}} e_{1}^{2} q_{1}\left(x_{1}\right) \bar{q}_{1}\left(x_{2}\right) d x_{1} d x_{2}
$$

$e_{i}$ is the parton charge in units of the electron charge, and

$q_{i}\left(x_{1}\right) d x_{1}$ and $\bar{q}_{1}\left(x_{2}\right) d x_{2}$ are the parton structure functions or the density of fractional momentum $x$, carried by quarks of type 1.

The quark structure functions are defined in terms of the deep inelastic neutrino-proton scattering structure. functions as

$$
\begin{aligned}
\text { and } & \sum_{1}\left[q_{1}+\bar{q}_{1}\right]=E_{2}^{u p} \\
\sum_{1}\left[q_{1}-\bar{q}_{1}\right] & =x F_{3}^{u p}:
\end{aligned}
$$

The orstructure functions used here are related, the commonly used parton distribution functions (the number density of quarks with fractional momentum $x$ ) by d

$q_{\text {Structure }}=x q$ Distribution. 
Summing over all partons in the hadrons, the cross section for muon pair production becomes

$$
\frac{d^{2} \sigma}{d x_{1} d x_{2}}=\frac{4 \pi a_{1}^{2}}{9 s x_{1}^{2} x_{2}^{2}} \sum_{1} e_{i}^{2} q_{1}\left(x_{1}\right) \bar{q}_{1}\left(x_{2}\right) .
$$

The sum includes all parton types and an explicit factor of $1 / 3$ has been included to take into account the requirement that the anninilating quarks must have the same colour quantum number'.

$\rightarrow$ Rather than using $x_{1}$ and $x_{2}$, it is conventional to express the cross section in terms of the equivalent variables,

$$
M^{2}=x_{1} x_{2} s
$$

the invariant mass of the muon pair, and Feynman $x$, defined as.

$$
x_{F}=p_{L} / p_{\text {Lmax }}
$$

the fraction of the maximum longitudinal momentum, possibleifor a muon pair in the hadron-hadron centre of mass system. The invariant mass of the muon pair is of ten expressed in terms of the dimensionless, ratio

$$
, \tau=M_{1}^{2} / s=x_{1} X_{2} \text {. }
$$

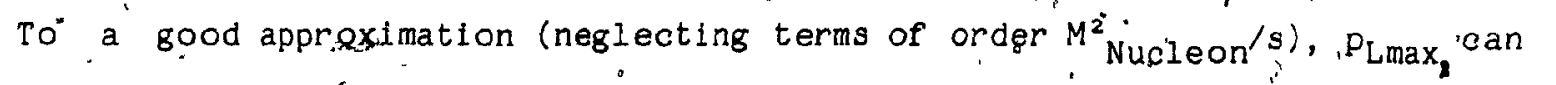
"be taken to be

$$
p_{\text {Lmax }}=\frac{\sqrt{s}}{2}(1-\tau)
$$

Using this approximation, Feynman $x$ becomes$$
x_{F}=\frac{2 p_{L}}{\sqrt{s(1-\tau)}}
$$

Since the muon pair may be produced with appreciable transvierse momeritum due to the intrinsic transverse momentum of the quarks or higher order effects ignored by the parton model, - an additional kinematic variable is necessary to completely specify the motion of the muon pair. The third kinematic variable is conventionally taken to be $\mathrm{p}_{\mathrm{T}}$ ', the component of' the . muon pair's momentum perpendicular to the hadron-hadron axis in the hadronic centre of mass frame, as given' by

$$
\overrightarrow{\mathrm{p}}_{\mathrm{T}}=\overrightarrow{\mathrm{p}}_{\mathrm{a}}+\overrightarrow{\mathrm{p}}_{\mathrm{b}}-\overrightarrow{\mathrm{p}}_{\mathrm{L}} \text {. }
$$


In terms of the variables $M$ and $x_{F}$, the variables $x_{1}$ and $x_{2}$ become

$$
x_{1}=\frac{1}{2}\left[\left(x_{F}{ }^{2}(1-\tau)^{2}+4 \tau\right)^{1 / 2}+x_{F}(1-\tau)\right]
$$

and

$$
x_{2}=\frac{1}{2}\left[\left(x_{F}{ }^{2}(1-\tau)^{2}+4 \tau\right)^{1 / 2}-x_{F}^{0}(1-\tau)\right]
$$

and the cross section (integrated over $p_{T}$ ) becomes

$$
\frac{d^{2} 0}{d M d x_{F}}=\frac{4 \pi \alpha^{2}}{9 M^{3}} \frac{2 \tau(1-\tau)}{\sqrt{x_{F}^{2}(1-\tau)^{2}+4 \tau}} \sum_{i} e_{i}^{2} q_{i}\left(x_{1}\right) \bar{q}_{i}\left(x_{2}\right)
$$

An immediate consequence of the parton model apparent froin this equation is that the cross section $M^{3} \frac{d^{2} o}{d M d x_{F}}$ scales, or depends only on $M$ and $s$ through the dimensionless ratio $\tau$. Integrating over the same $x_{F}$ region, the cross sections $M^{3} \frac{d o}{d M}$ and $s^{3 / 2 d o} \frac{d M}{d M}$ should be functions of only $\tau$ and not $M$ and $s$ separately. Likewise the cross section $s \cdot \frac{d \sigma}{d^{2}}$ should be independent of $M$ and $s$ if integrated over the same region in $\tau$, allowing data at different beam energies to be compared.

\subsection{Angular Variables}

The variables $M, x_{F}$ and $P_{T}^{2}$ completely specify the motion of the rest frame of the muon pair with the exception of a trivial azimuthal angle about the hadron-hadron axis. Two additional variables are necessary to specify the motion of the individual muons with respect to the muon pair rest frame. These are typically taken to be $\theta$, the polar angle, and $\phi$, the azimthal. angle of the positive muon in the rest frame of the muon pair. Ideally the angles would be measured from the axis defined by the annihilating partons. Because the constituents are not necessarily collinear with the incoming hadrons, and because higher; order effects can contribute to the cross section, the muon pair generally has some transverse, momentum in the 


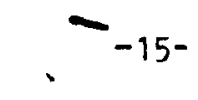

hadron-hadron centre of mass system. When the particles are transformed to the muon pair rest frame, the beam and target momenta are not collinear and the parton-parton axis is unknown. In practice a convention is chosen such 3 that the $Z$ axis colncides with the hadron-hadron axis if the transverse momentum of the muon pair is zero.

The angles are shown schematically in Figure 3 . Working in the rest frame of the muon pair, the beam and target momenta define a plane, conventionally taken to be the $x-z$ plane. The $Y$ axis is taken to be the unit normal to the plane in the direction of $\vec{p}_{a} \times \vec{p}_{b}$. The direction of the $z$ axis depends on a choice of convention. Two choices commonly used are the Gottfreid-Jackson[23] and" the Collins-soper frames[24]. The angle between two hadrons in the muon pair rest frame depends only on the hadronic "variables, $M, x_{F}$ and $p_{T}$. If we call the angle between the $z$ axis and the beam momentum, $\alpha$, then the Gottfreid-Jackson frame corresponds to a choice for $a$ of

$$
\alpha_{G-J}=0
$$

whereas the Collins-Soper frame corresponds to a choice for $\alpha$ of

$$
\alpha_{\mathrm{C}-\mathrm{S}}=\left(\pi-\theta_{\mathrm{H}}\right) / 2
$$

The Collins-soper frame represents an attempt to take - the intrinstc transierse momenta of the partons into account on the average under 'the assumption that the transverse momentum distributions of partons in the beam

: and target particles are the same.

Lam and Tung[25] have shown that if the process is mediated by a single photon, then the distribution of muons in the rest frame of the pair, must be

$$
\begin{aligned}
& \text { of the form } \\
& \qquad \begin{aligned}
\frac{1}{\sigma} \frac{d \sigma}{d \Omega} & =\frac{3}{8 \pi}\left[W_{T}\left(1+\cos ^{2} \theta\right)+W_{L}\left(1-\cos ^{2} \theta\right)+\right. \\
& \left.W_{\Delta} \sin 2 \theta \cdot \cos \phi+W_{\Delta \Delta} \sin ^{2} \theta \cdot \cos 2 \phi\right] /\left[2 W_{T}+W_{L}\right] .
\end{aligned}
\end{aligned}
$$




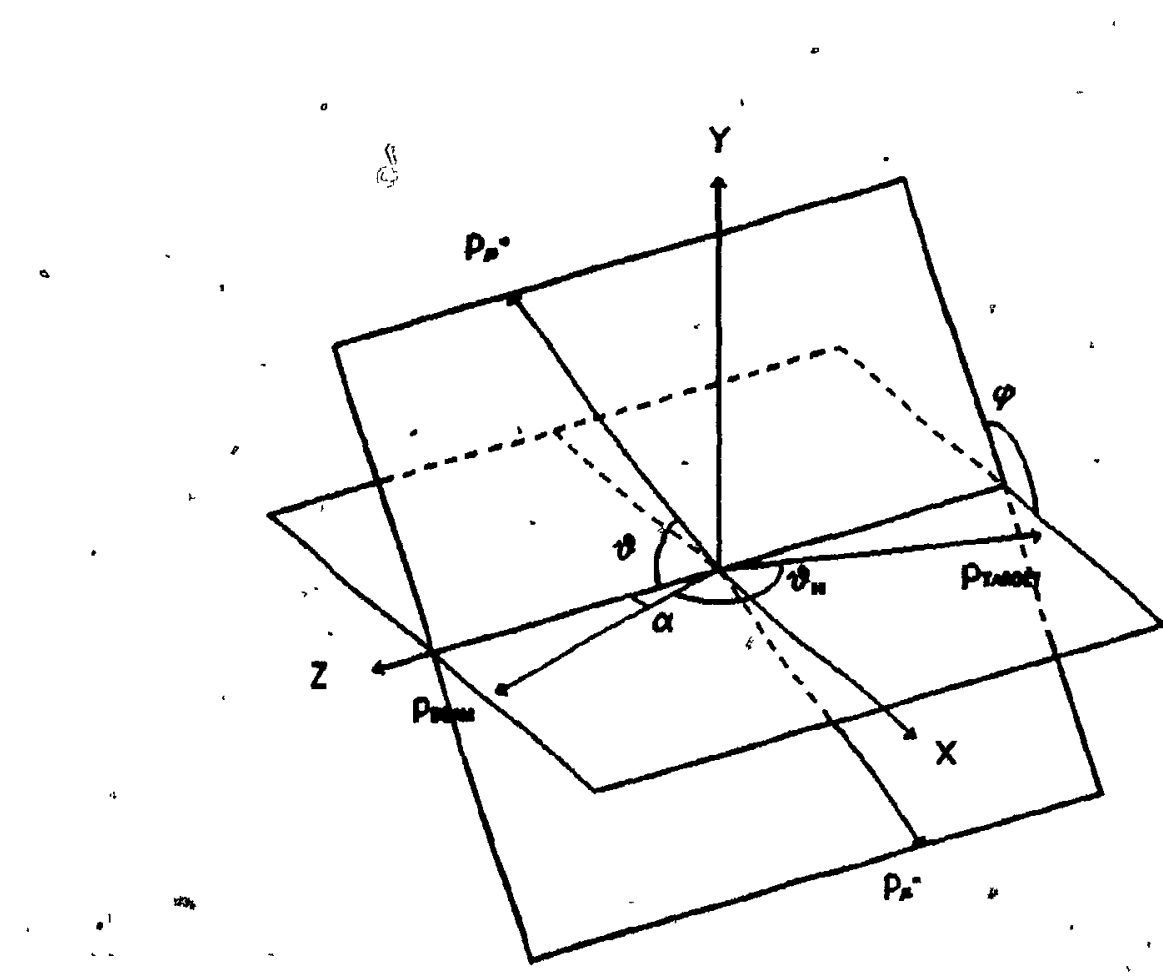

Elgurè 3 - Angular Variables

The angular variables are defined in the rest frame of the muonipar. The beam and target particle momenta define the $X-Z$ plane. The $Y$ axis is defined by the normal to the $x-z$ plane. The cholce of $z$ axis depends on the convention chosen as discussed in the text. The angles $\theta$ and $\phi$ are the polar and azimuthal angles with respect to the $Z$ axis.

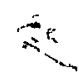


where $w_{T}, w_{L}, w_{\Delta}$, and $w_{\Delta \Delta}$ depend on the nature of the constituents. In the partion model plcture, the angular distribution of muons in the rest frame of the virtual photon should be

$\therefore \frac{1}{0} \frac{d \sigma}{d \Omega}=\frac{3}{8}\left[1+\cos ^{2} \theta\right]$,

and independent of $\phi$, if the process is the result of the electomagnetic annihilation of point-like spin $1 / 2$ constituents. A first order QCD calculation by Colilins[26] shows that for interactions dominated by. valenge-valence subprocesses the terms other than that involving " $1+\cos ^{2} \theta$

should be small.

\subsection{Quantum Chromodynamics}

Perturbative corrections to the Drell-Yan model can be calculated using Quantum Chromodynamics. Subprocesses such as those shown in Figure 4 can produce a massive photon which decays to a muon pair. In the impulse approximation, the cross sections for the subprocesses are calculated assuming that the quarks and gluons are free. The cross sections are convoluted with the quark and gluon structure functions of the beam and target particles. Calculations of the corrections to first order contain both infrared and mass singularities. The infrared singularities are handled as in Quantum Electrodynamics.

Politzer[27] pointed out that at the one loop level, the mass singularities are similar to the singularities that arise in the one loop corrections to deep inelastic scattering. In deep inelastic scattering, the logarithmic singularities can be absorbed into the structure functions at a particular value of $Q^{2}$ by defining an unobservable bare structure function 


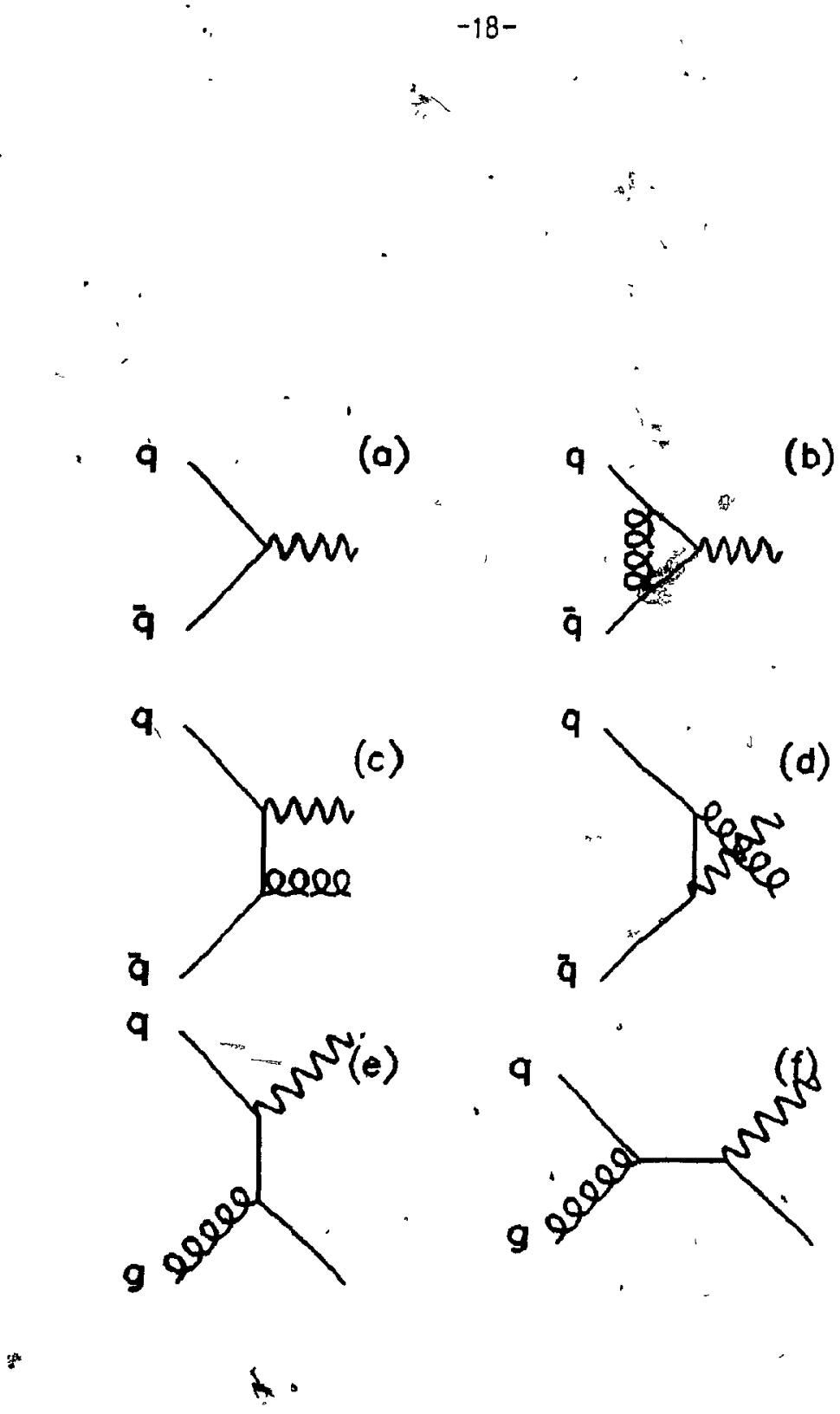

Figure 4 - QCD Annihilation and Compton Processes

Higher order QCD subprocess can also contribute to muon pair production. The Drell-Yan subprocess is shown in (a). A quark (single line) in the beam particle anninilates with an antiquark in the target particle to create a massive photon (wavy line), which can decay to give a pair of muons (not shown). QCD vertex corrections, such as (b), lead to large constant corrections. Anninilation diagrams such as $(c)$ and $(d)$, involving a gluon (looped line) in the final state, and Compton diagrans such as (e) and ( $f$ ), involving a gluon in the initial state, can give the muon pair appreciable transverse momentum. 
$q_{0}$, which is also ingular, in such a way as to cancel the singularities of the correction term. The observable structure functions are now finite, but depend logarithmically on $Q^{2}$. The similarities of the one loop corrections suggested that thè Drell-Yan cross section can still be expressed in terms. of deep inelasticistructure functions if the structure functions depend explicity on $Q^{2}$, that is,

- $q(x) \rightarrow q\left(x, Q^{2}\right)$,

where $Q^{2}$ is identified as $M^{2}$ :

Calculations in the leading $\log$ approximation[28] and to first order in the stong coupling constant, $\alpha_{s}[29][30] ;$ have confirmed that the singularites can be cancelled in this way. The size of the first order corrections has been shown to be of the same order as the parton model cross sections, that is,

$$
\frac{d^{2} 0}{d M d x_{F}}=K\left(\frac{d^{2} 0}{d M d x_{E}}\right)_{\text {Parton }}
$$

where

$$
K=1+\frac{\alpha_{s} \pi^{2}}{2 \pi 3}
$$

and does not depend strongly on $M$ or $x_{F}$.

The size of the first order terms has raised concerns about the validity of the perturbative approach. It has been conjectured, on the basis of an analogy with QED, that the first order corrections are the leading term in the series expansion of an exponential[31].

$$
K \rightarrow \exp \left[\frac{\alpha s}{2 \pi} \frac{4 \pi^{2}}{3}\right]
$$

Calculations up to order $a_{s}^{2}$ included (two loop level), have confirmed[32][33] that corrections to the Drell-Yan process lead to a well. defined part of the cross section giving the first three terms in a series expansion of

$$
-\exp \left[\frac{\alpha_{3}}{2 \pi} \frac{4 \pi^{2}}{3}\right]
$$


Measurements of the cross section in proton-nucleon and pion-nucleon collisions seem to confirm that the measured cross sections are in fact -... about twice as large as the parton model would predict, but in both cases the calculations are dominated by terms involving structure functions which are not weli known. Proton-nucleon muon pair production is dominated by the annihilation of valence quarks in the beam particle with the sea quarks of . the target particle. Inelastic scattering experiments are able to measure the distributions of the valence quarks but the extraction of the sea quark distributions is sensitive to the assumption"s made.

The pion structure functions are not accesible to electron scattering experiments. Muon and electron pair production may provide the best way to measure them. Because the normalization of the cross sections is very sensitive to the behaviour of the structure functions at low $x$, a region which is masked by resonances and other backgrounds, the normalization of the pion-nucleon Drell-Yan cross section. is subject to large systematic uncertainties.

Measurements of the antiproton-nucleon cross section for muon pair production provides the best way to measure the $\mathrm{K}$ factor. The process is dominated by the anninilation of valence anti-quarks in the antiproton with valence quarks in the nucleon. The antiproton valence structure functions must be the same as the proton structure functions because of particle-antiparticle symmetry. and the nucleon valence structure functions are well measured in electron, muon and neutrino scattering experiments gver. large ranges of $x$ and $Q^{2}$. 


\subsection{Nuclear Dépendence}

$\infty$

The question of the nuclear dependence of the cross sections ils one of considerable practical importance but difficult to treat theoretically. Experiments are forced to use heavy nuclear targets in order to achieve an acceptable, event rate and must extrapolate to obtain a cross section per nucleon.: Nuclear effects are inherentiy nonperturbative, making them difficult, if not impossible, to calculate. The nuclear dependence is usually empirically parameterized as

$$
0=\sigma_{0} A^{\alpha}
$$

and measurements using several nuclear targets are fit to obtain $\alpha$ and $0_{0}$. If thé nuclei are not isoscalar, $o_{0}$ will be a function of $Z / A$, the ratio of the atomic number and the atomic mass.

It can be argued that a strong process will involve interactions at the 5 surface of the nucleus and should depend on $A$ as

$$
\dot{0}=\sigma_{0} A^{2 / 3}
$$

whereas a weaker probe like the photon will have' an A. dependence proportional to the volume of the nucleus and

$$
0=O_{0} A^{1} \text {. }
$$

Currently accepted values [34] for $\alpha$ are consistent w1 th

$$
\alpha_{D Y}=1 \cdot 0
$$

Recent results from muon scattering experiments at CERN[35] and a. reanalysis of electron scattering results at SLAC[36][37] have shown that the structure functions of nucleons bound in iron and aluminum nuclei are not, the same as the quasi-free nucleons of deuterium. Since most of the 
structure function measurements use Iron, which should be similar to our tungsten target, and since our experiment does not have sufficient data to - see the difference betwęen the structure function of the free antiproton and the nucleons of a tungsten nucleus, we have ignored these differences in our analysis.

\subsection{Previous Experiments}

Muon pairs were first observed at Brookhaven in 1970 by a Brookhaven-Columbia-Stony Brook (BES)[1] collaboration using a proton beam and a uranium target to look for w bosons. A Columbia-Fermilab-Stony Brook (CFS) [38] collaboration at Fermilab, using a double arm spectrometer studied both muon and electron pair production in proton-nucleon colissions. Work by the same group led to the discovery of the upsilon family of resonances. A Chicago-Illinois-Princétion (CIP) [39] collaboration working at Fermilab at about the same time made the first attempt to extract the pion structure function[40]. Measurements of the $\pi^{+} / \pi^{-}$cross section ratio from nuclear targets by this group, provided confirmation of the electomagnetic nature of the production process.

Several experiments at CERN have published important results in the last several years. The Omega[41][42][43] collaboration has made measurements of the cross section for muon palr production at $40 \mathrm{GeV} / \mathrm{c}$, with pion, kaon, proton and antiproton beams. As well as demonstrating the beam dependence of the cross section; comparison of the Omega data with experiments at higher energies provides one of the best'examples of the type of scaling behaviour expected from the Drell-Yan model. The GOLIATH[44] collaboration measured muon and electron pair production rates in 
pion-nucleon collisions at beam energies of 150 and $175 \mathrm{GeV} / \mathrm{c}$. The NA3[45][46][47] collaboration at CERN has made extensive measurements of muon pair cross sections with proton, pion, and kaon beams incident on a platinum target as well as the flrst antiproton[10] results. Measurements by the NA3 group of the $\pi^{+} / \pi^{-}$cross section ratio for tungsten and hydrogen targets[48], measurements of the angular distributions of the muon pairs[49]

- and measurements of the A dependence of the muon pair cross section[50] have confirmed many of the features expected from the Drell-Yan model. The NA10[51] experiment has published preliminary results using an intense pion beam to produce muon pairs in a tungsten target. Initial results from this experiment[52] provide confirmation of the linear A dependence measured by the NA3 collaboration. Detailed comparisons of our data to data obtained by the NA3, CIP, Goliath and Omega collaborations will be made in the final chapter.

1

Two experiments running currently at Fermilab are also studying muon pair production. An extension of the CFS collaboration[53] is looking at the production of high mass electron pairs in proton-nucleon collisions at beam momenta of up to $1 \mathrm{TeV} / \mathrm{C}$ with a new spectrometer in the Meson Area. Members of the CIP collaboration[54] are examining muon pairs produced in plon-nucleon collisions at very high $x_{1}$ across the range of cose, using an apparatus in the High Intensity Area of the Protion line, upstream of the . spectrometer used for this experiment. 
CHAPTER 3

\section{Apparatus}

The experiment described here was located in the high intensity area of the west proton beam line at Fermilab, in Batavia, Illinois, and took data in the spring of 1981 and the winter of 1982. The apparatus used was $=$ designed to look for events with one beam particle hitting the target, and a pair of muons leaving it. Systems of Cerenkor and 'scintillation counters with good time resolution provided fast signals to trigger the apparatus and reject accidental coincidences and background. Proportional and drift chambers with good spatial resolution provided precise measurements of points along the particle trajectories so that the events could later be reconstructed of fline.

The apparatus used for the experiment was a closed geometry magnetic spectrometer, shown schematically in Figure "5. Tungsten; copper, and beryllium targets were exposed to a beam of $125 \mathrm{GeV} / \mathrm{c}$ pions and antiprotons. 


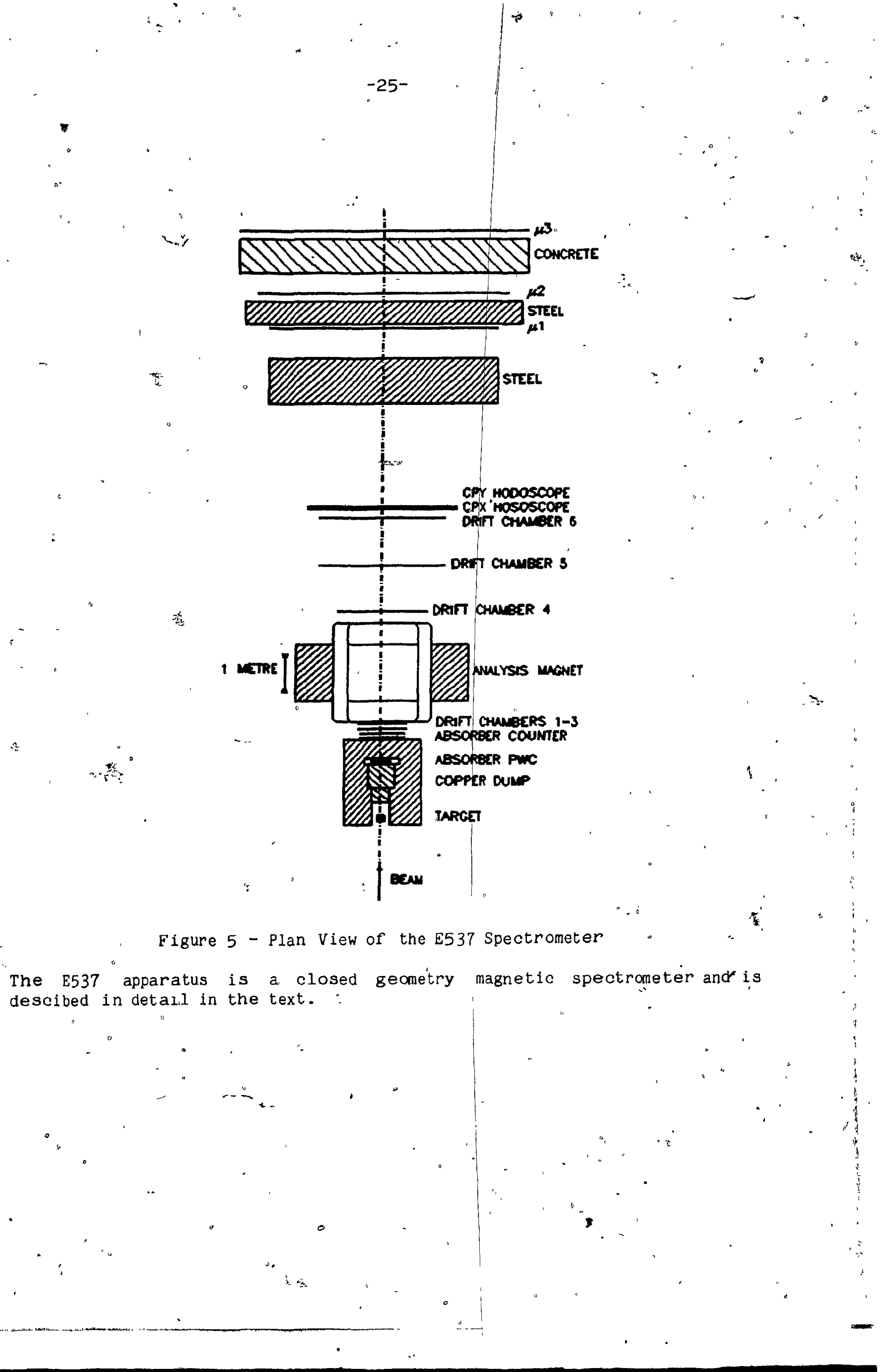


w

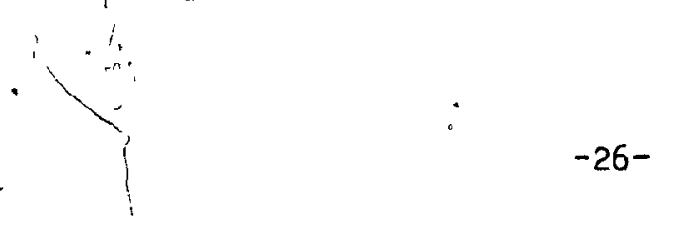

Hadrons from the interactions were filtered out by a copper beam dump. Track copordinates were measured $2 / 3$, of the way through the beam dump by two lpianes of proportional, wires. Immediately downstream of the dump a scintiplation, counter designed to detect.muons produced at a large angle with respect to the beam provided a high mass bias for the fast trigger. Trlajectorles were also measured downstream of the dump by 9 planes of drift chambers. A large conventional dipole magnet deflected charged particles to permit a momentum measurement. Particles were tracked downstream of the magnet by 9 plänes of drift chambers and 2 scintillation counter hodoscopes. Muons were identified by requiring them to pass through walls "of steel and concrete interleaved with'scintillation counter hodoscopes. Muons required a momentum of $6 \mathrm{GeV} / \mathrm{c}$ to penetrate to the final hodoscope. Signals from the counters-were combined to praduce a fast trigger/stgnál when two muons were detected. "liow mass events were rejected by a fast trigger processor, and "information from the cqunters and chamber wires was written to magnetic tape $30^{\circ}$ that the kinematic. variables of the muon pair could be reconstructed of filine.

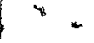

A sample of $4.0 \times 10^{5}$ dimuon events with mass greater thän $2 \mathrm{GeV} / \mathrm{c}$ was collected in a running period of 13 weeks.

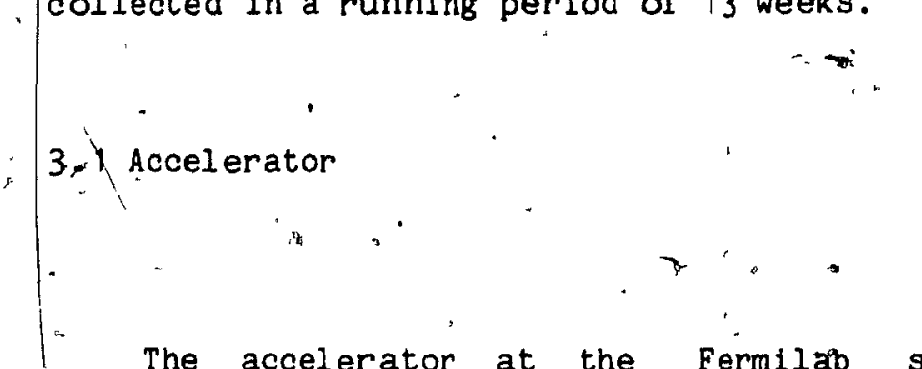

The accelerator at the Fermilab site is a $400 \mathrm{GeV}$ proton synchrotron[55]. Protons from hydrogen gas source are given a kfnetic energy of $750 \mathrm{KeV}$ by a Cockroft-Walton electrostatic accelerator. A $200 \mathrm{MeV}$ Iinac injects the protions into an $8 \mathrm{GeV}$ booster syncrotron from which they enter the main ring. One burst of up to $3.0 \times 10^{13}$ protons is accelerated. 
every 9 to 15 seconds. The beam is extracted in a 1 seoond spill and split three ways to the Meson, Neutrino and Proton lines. The beam particles are not uniformly distributed thoughout the spill but cluster in buckets spaced 19 ns apart. The interbucket spacing is characteristic of the radio frequency voltage used in the main accelerator. Timing signals from the main control room allow the experiment to be synchronized with the

' accel erator.

\subsection{Antiproton Beam}

A schematic layout of the peam line elements is shown in Figure 6. A neutral beam of lambdas and kaons was produced by $400 \mathrm{GeV} / \mathrm{c}$ protons striking a $15 \mathrm{~cm}$ beryllium target[56][57]. Charged secondary particles were removed from the beam by a dipole sweep ing magnet $2.3 \mathrm{~m}$ downstream of the primary target. Pions and antiprotons from the decay in floht of the neutrals were gathered by a flux collecting quadrupole triplet and pransported to the experimental hall by a beam line of conventional dipoles and quadrupoles. A dipole and collimator selected beam particles with a momentum of $125 \mathrm{GeV} / \mathrm{c}$ and eliminated the positive decay products. A FODO channel collected the particles and transported them to the final focussing triplet. Electrons were removed from the beam by a $2.5 \mathrm{~cm}$ thick lead absorber.

The resulting beam consisted of 79 percent pions and $21^{\prime}$ percent antiprotons, with a 10 percent momentum bite FWHM. The angular and spatial diyergences' of the beam at the experimental target were $1 \mathrm{mr}$ and $2.5 \mathrm{~cm}$ respectively. To reduce uncertainties due to the large phase space of the beam, the momentum and trajectory of each individual beam particle was tagged in the experimental hall by a system of proportional wire chambers 


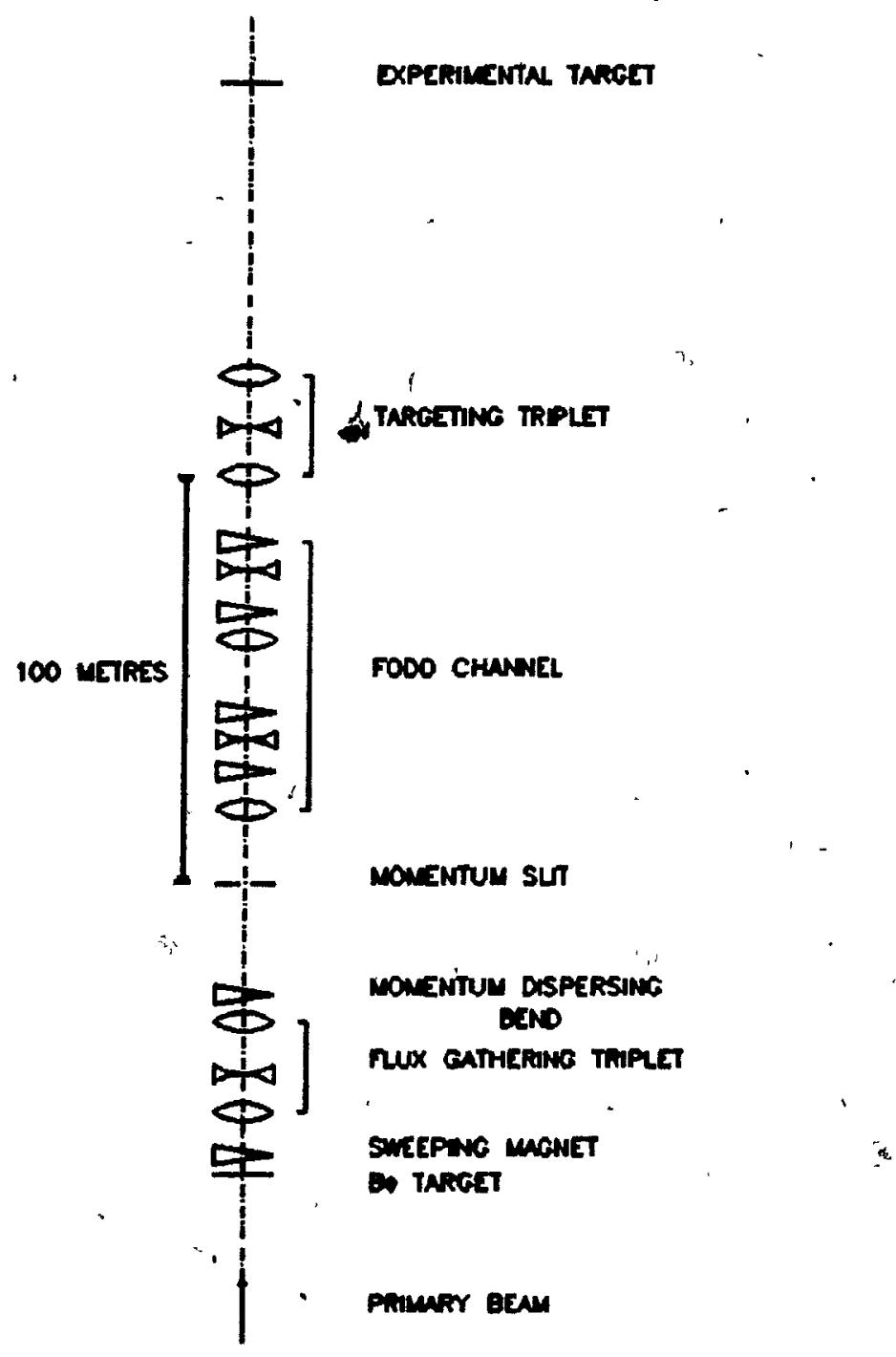

Figure 6- High. Intensity Ạrea Beam Line

A primary beam of $400 \mathrm{GeV} / \mathrm{c}$ protons strikes the beryllium target. Charged secondaries are swept from the beam and the neutral secondarles allowed to decay. A dipole and slit select $125 \mathrm{GeV} / \mathrm{c}$ pions and antiprotons from the decay products and a string of conventional dipolgs and quadrupoles
transports them to the experimental target.

. 7

in


and bending magnets described in the next section.

The primary beam flux was monitored by two Secondary Emission Monitors $\left(\mathrm{SEM}^{\prime} \mathrm{s}\right)[58]$ upstream of the primary target. Beam position and spot size were monitored along the beam 1 ine and in the experimental hall by Segmented Wire Ionization Chambers (SWIC's)[59][60] tied into the Fermilab beam line control system. A primary beam flux of $7 \times 10^{12}$ protons/spill typically resulted in a secondary beam flux of $12 \times 10^{6}$ particles/spill.

The beam was surrounded by a halo of muons produced by decaying pions and energetic secondary particles not removed from the neutral beam. Because the accidental coincldence between halo particles and a single muon produced by the beam could mimic the signature of a muon pair, thus increasing the trigger rate and contaminating the data fsample, steps were taken to suppress as much of the halo as possible. Muon spoiler magnets upstream of the experimental hall swept of axis particles, away from the beam region. The veto counters used to inhibit the apparatus when a'halo particle was present are discussed in, a later section. Strict vertex requirements, imposed on the events offline to suppress any remaining. contamination of the data sample, are discussed in the next chapter.

\section{,3.3 Beam Tagging}

8

The momentum and trajectory of each beam particle was measured with a

- beam telescope consisting of 9 planes of proportional chambers, three $Y$ hodoscopes and two small dipole magnets. Beam particles were Identifled by : two Cerenkov counters which will be disoussed in the next section. 
A plan view of the beam tagging system showing the relative locations of the elements is given in Figure 7 . The first beam station, BY1, which

1. consisted of three planes of proportional chambers and a hodoscope followed the first Cerenkov counter, CS1. A second beam station, BY2, was placed 3.95 meters downstream of the second Cerenkoy counter, CS2. A third beam chamber station, BY3, followed $3.43 \mathrm{~m}$ upstream of the experimental target. " The pair of magnets between CS2 and the second beam station gave each beam particle a vertical momentum increment of $0.319 \mathrm{GeV} / \mathrm{c}$, allowing a beam . particle with a momentum of $125 \times \mathrm{GeV} / \mathrm{c}$ to be measured to an accuracy of $1 \mathrm{GeV} / \mathrm{c}$.

- Each beam station had three planes of proportional wire chambers, with the wires oriented along the $U, V$ and $Y$ directions. The $Y$ coordinate was taken in the vertical direction with the $U$ and $V$ directions defined by clockwise rotations about the $\mathrm{Z}$ axis (beam direction) of 240 and 120 degrees. from $Y$ respectively. Each station had an assoclated scintillator hodoscope which measured the $Y$ coordinate.

All beam chamber sense wire planes consisted of 128 wires of $12.5 \mu$ dlameter spaced $1 \mathrm{~mm}$ apart. Cathode planes were made using $25.4 \mu$ thick pluminum foll. The chambers were operated using a gas mixture of 25 percent 1sobutane, 5 percent methyilal, 0.5 percent freon with the balance being argon. A discrete component amplifier followed, by a high speed ECL comparator provided a differential time over threshold output[61].

Each hodoscope consisted of a 15.24 cm diameter disk of $0.6 \mathrm{~cm}$ thick'. NE110 plastic scintillator[62] segmented horizontally into eight parts. Each segment was optically coupled to a 12 stage Amperex 56AvP 


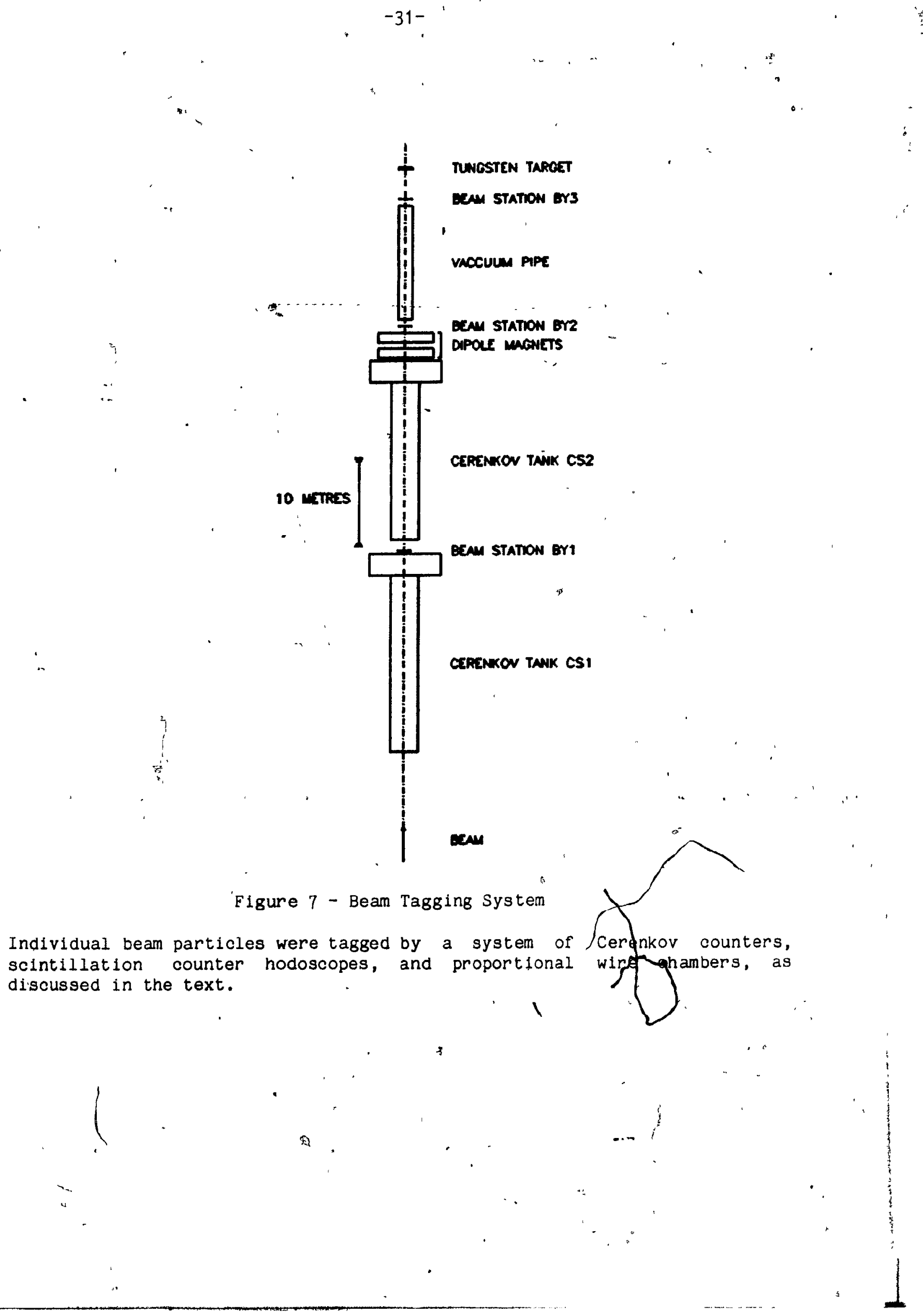


photomultiplier[63] with a resistive voltage divider base. Zener diodes were used to clamp the final two dynode voltages. Photamultiplier outputs were clipped using a $3 \mathrm{~ns}$ stub of $50 \Omega$ soaxial cable terminated in $25 \Omega$ to allow the highest possible counting rates". The widths of the hodoscope segments were chosen to roughly equalize the individual counting rates.

The proportional chambers gave an accurate measurement of the position of the beam particle trajectory but were sensitive for a period of $60 \mathrm{~ns}$, which included beam particles in three separate accelerator RF buckets. The beam hodoscope elements were designed to have a resolving time of $10 \mathrm{~ns}$, and were capable of distinguishing between beam particles in successive $R F$ buckets. Discriminator signals fram the beam hodoscopes were recorded with each event so that tracks not associated with the beam particle causing the trigger could be rejected by the of fline analysis.

\subsection{Cerenkov Counters}

The type of each incident beam particle was established by two differential gas Cerenkov counters. Both counters were filled with a mixture of 80 percent hel lum and 20 percent nitrogen. The fraction of beam particles counted by the first Cerenkov counter as a function of gas pressure is shown in Figure 8. Between pressures of 3 and 6 PSIA the counter is below. the antiproton Cerenkov threshold and counts only. pions, which constitute 80 percent of the beam. Between 10 and 12 PSIA, Cerenkov light from the pions is at the wrong angle to hit the photomultiplier and the counter is only sensitive to antiprotons, the remaining 20 percent of the beam. The first counter, CS1, was set to count antiprotons at 10.5 PSIA

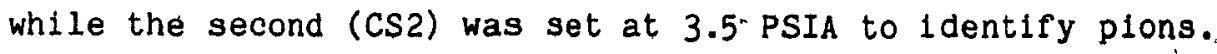




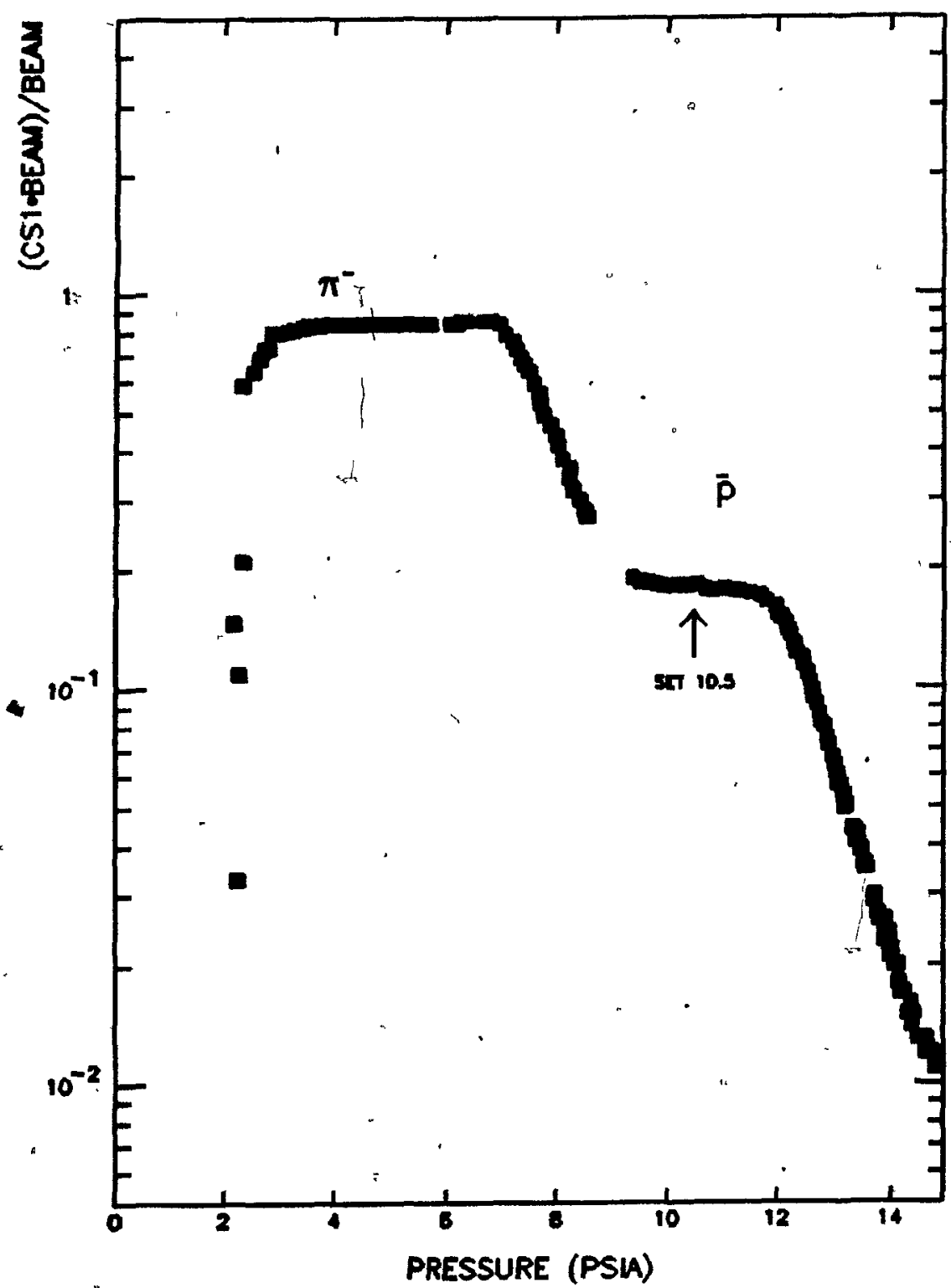

\section{Figure 8 - CS1 Cerenkov Counter Pressure Curve}

The vertical axis shows the fraction of the beam particles detected by the first Cerenkov counter. Between gas pressures of 3 and 6 psia the counter is below the antiproton Cerenkor threshold, and counts only pions. Above 10 psia the counter is not sensitive to light in the pion Cerenkov cone and the counter detects only antiprotons. 


\section{$-34-$}

Cerenkov light pröduced in the $22 \mathrm{~m}$ long counters was reflected by a $33 \mathrm{~cm}$ diameter plastic mirror onto an RCA 31000M[64] photomultiplier tube through a $7.62 \mathrm{~cm}$ diameter quartz window. The mirrors were made of aluminized lucite with a $4.572 \mathrm{~m}$ radius of curvature and a $2.286 \mathrm{~m}$ focal length. Annular masks on the photomultiplier windows restricted the sensitivity of the counters to a cone of light with half angle between 4.4 $\mathrm{mr}$ and $7.9 \mathrm{mr}$ in the case of CS1 and $5.7 \mathrm{mr}$ to $7.6 \mathrm{mr}$ in the case of CS2.

The counters were designed to be able to distinguish between beam particles in successive RF buckets. Transistorized bases[65] permitted counting rates in excess of $3.0 \times 10^{7}$ particles/second. Photamultiplier signals were clipped by a 3 ns stub of $50 \Omega$ cable terminated in $25 \Omega$. The $25 \Omega$ termination damped the $\therefore$ the counters an output.pulse of less than 6 ns duration with no ringing or overshoot. Signals were amplified by Lecroy VV100B $10 \times$ amplifiers[66] prior to discrimination to reduce the average anode currents in the tubes. The average number of photoelectrons per particle ranged from 7-10 and the discriminators were set to trigger at 2 or more.

Cerenkov counter efficiency was monitored during the data taking by comparing the number of beam particles counted by the Cerenkov counters to the number of beam particles counted by the beam telescope, using - CERENTOT $=[($ CS1 BEAM $)+($ CS $2 \cdot B E A M)-(C S 1 \cdot C S 2 \cdot B E A M)] / B E A M ;=$ where

(CS1-BEAM) is the number of coincidences between signals from the first Cerenkov counter (CS1) and the beam telescope (BEAM), that' is, the number of beam particles, counted by the antiproton Cerenkov counter, 


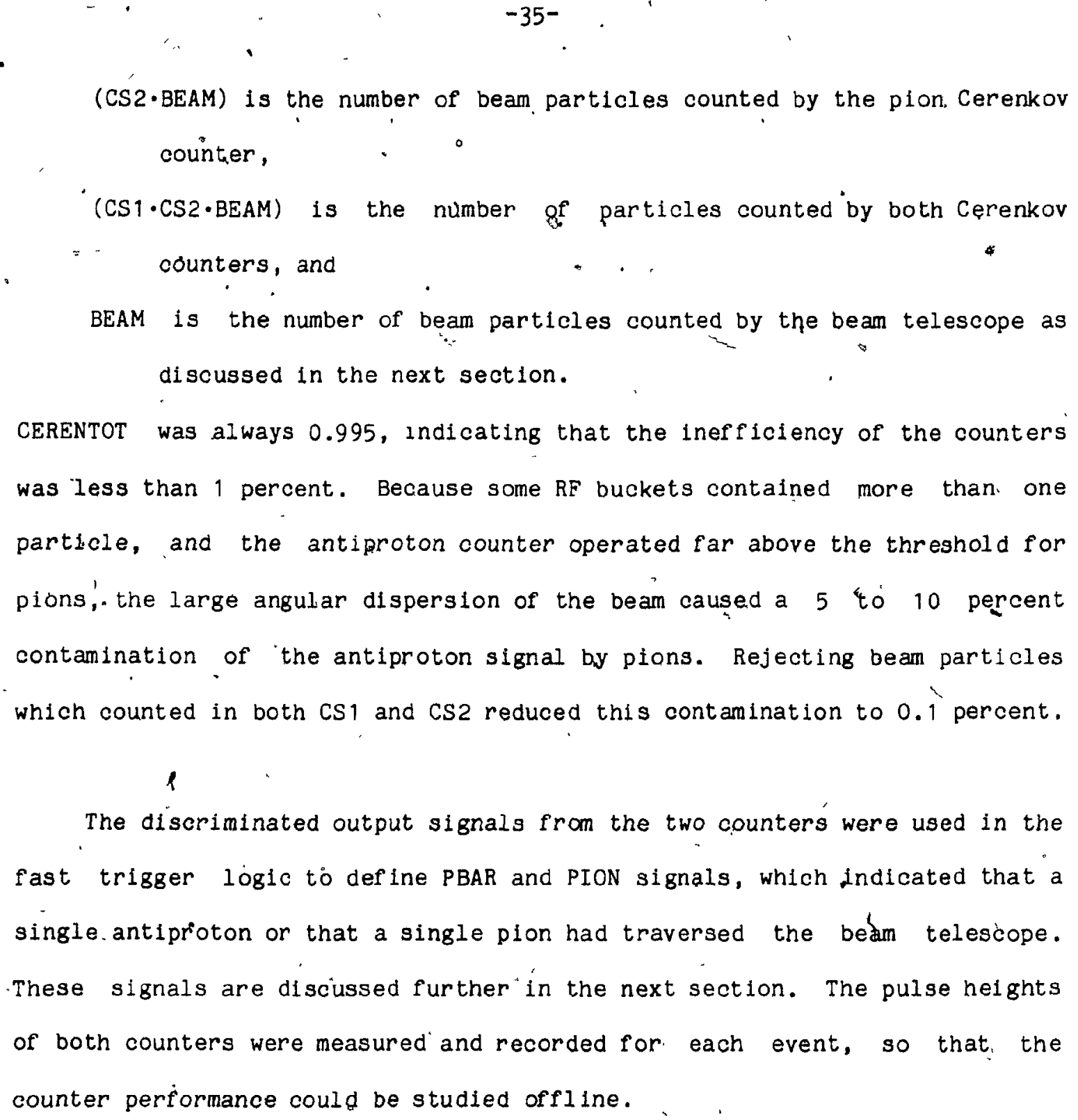

(CS2.BEAM) is the number of beam particles counted by the pion. Cerenkov counter,

(CS1·CS2-BEAM) is the number of particles counted by both Cerenkov counters, and

BEAM is the number of beam particles counted by the beam telescope as discussed in the next section.

CERENTOT was always 0.995 , indicating that the inefficiency of the counters was less than 1 percent. Because some RF buckets contained more than. one particle, and the antiproton counter operated far above the threshold for pions,'. the large angular dispersion of the beam caused a 5 to 10 percent contamination of the antiproton signal by pions. Rejecting beam particles which counted in both $\mathrm{CS} 1$ and CS2 reduced this contamination to 0.1 percent.

\section{1}

The discriminated output signals from the two counters were used in the fast trigger logic to define PBAR and PION signals, which indicated that a single antiproton or that a single pion had traversed the bedm telescope. -These signals are discussed further in the next section. The pulse heights of both counters were measured and recorded for each event, so that the counter performance could be studied offline.

3.5 Beam Tagging Logic.

Standard NIM[67] logic modules were used to form a BEAM signal from the discriminated outputs of the beam hodoscope counters. The BEAM signalfiwas combined with signals.from the Cerenkov counters to indicate the presence of a beam particle, to the fast trigger logic. "A simplified schematic diagram of the beam logic is shown in Eigure 9. The logical on of the signals from the counters in the first beam hodoscope, BY1-1 through BY1-8, was used to 

define a signal, BY1, which indicated that a beam particle had passed through the first beam station. Signals from the counters in the second and third beam hodoscopes were similarly used to def ine the signals BY2 and BY 3 . The discriminated signals from all hodoscope elements were recorded for each event.

To Indicate when more than one beam particle had travelled through the beam telescope at the same time, the linear sums of the logical signals from the counters in each of the beam stations were rediscriminated to define the signals $B Y 1 \geq 2$, BYZZ2 and $B Y 3 \geq 2$ if two or more of the counters in the respective hodoscopes had been hit. These signals were combined to give a veto signal, $2 B Y \geq 2$, if at least 2 out of 3 of the beam hodoscopes had more than 2 elements hit.

A BEAM signal was defined as the coincidence between the BY1, BY2 and BY3 signals from the beam hodoscopes, the anticoincidence of the $2 B Y \geq 2$ veto signal, and the anticoincidence of a HALO signal formed from the outputs of the halo counters as described in the next section, that is,' $B E A M=B Y 1 \cdot B Y 2 \cdot B Y 3 \cdot \overline{2 B Y \geq 2} \cdot \overline{H A L O}$.

The beam signal was.TRUE. If at least one counter was hit in each of the three beam stations, at least two of the three beam. stations had only one counter hit, and none of the possible combinations of halo counters were hit. This signal indicated to the fast loglo that one, and only one, beam particle had passed through the beam telescope, and that there were no halo particles present.

The logical signals PBAR and PION were defined if there. Were both a beam particle and a signal from the corresponding Cerenkov counter as PBAR $=$ CS1 $\cdot$ BEAM $\cdot(\overline{\operatorname{CS} 1 \cdot \operatorname{CS} 2)}$, 
and

$\mathrm{PION}=\mathrm{CS} 2 \cdot \mathrm{BEAM} \cdot(\overline{\mathrm{CS} 1 \cdot \mathrm{CS} 2)}$,

where CS1 and CS2 were the discriminated'signals from the Cerenkov counters described in the last section.

The PBAR and PION signals were prescaled separately and then combined to give a $\pi^{-/ \bar{p}}$ signal which indicated the presence of a single identified. beam particle to the fast.logic. The antiproton prescaler was always set to count for each event but the pion prescaler was set to count once for every 1 to 4 events depending on overall trigger rate and beam conditions.

\subsection{Veto Counters}

Muons in the halo around the beam could combine with debris from interactions of the beam in the target and the dump to mimic the esignature of a high mass muon pair. To reduce the effects of beam halo on the trigger rate, vertical (VX) and horizontal (VY) counters positioned aroung the beam axis as shown in Figure 10, were used to define a HALO signal which inhibited the apparatus during the presence of beam halo.

The counters were constructed of $1 \mathrm{~cm}$ thick NE114 plastic scintillator[62] and instrumented with Amperex 2212B photomultipliers[63]. Discriminated signals from an inner array of eight $73.66^{\circ} \mathrm{cm} \times 12.7$, cm and twelve $147.3 \mathrm{~cm} \times 25.4 \mathrm{~cm}$ counters in the VX array were combined to form a VX signal as shown in Figure 11. A VY signal was formed with the OR of the discriminated signals from the eight $101.6 \mathrm{~cm} \times 12.7 \mathrm{~cm}$ and eight $152.4 \mathrm{~cm} \times 25.4 \mathrm{~cm}$ counters in the VY array. A VI signal formed from the AND of the $V X$ and the $V Y$ signals indicated that a particle had passed 


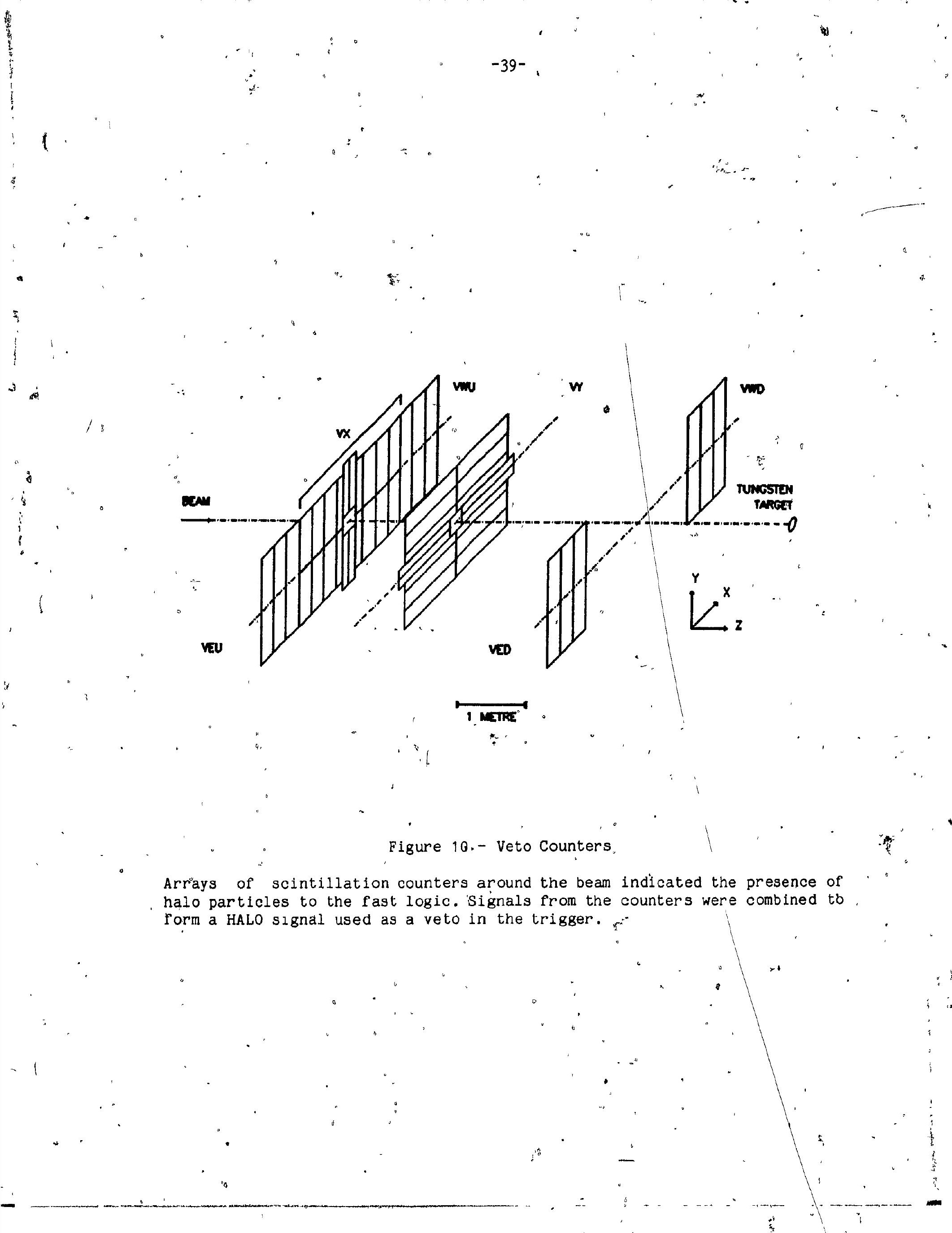




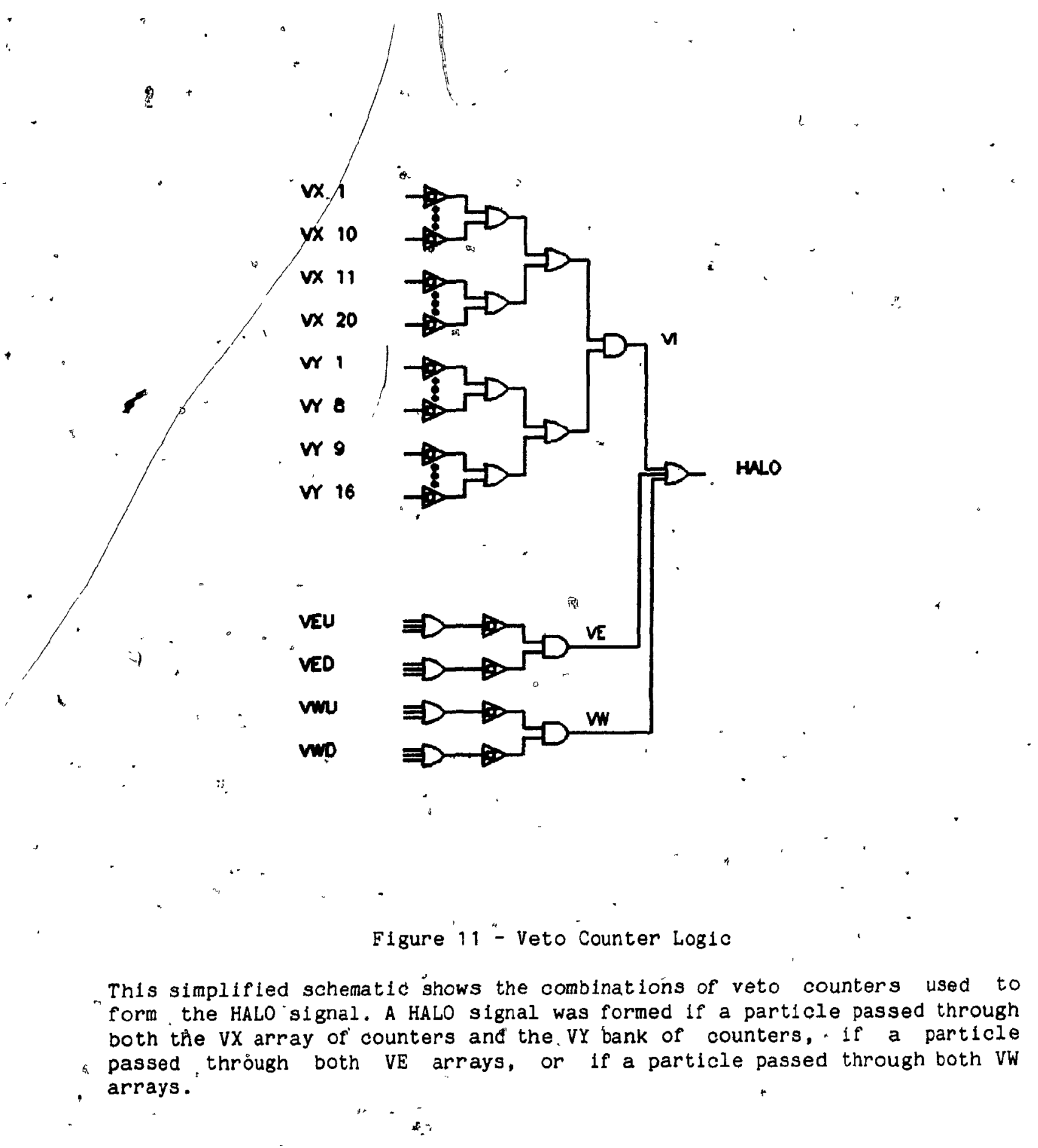


through both of the inner arrays of halo counters. Central counters in both the $V X$ and $V Y$ arrays were retracted from the beam axis to leave a $25.4 \mathrm{~cm}$ squáre beam hole.

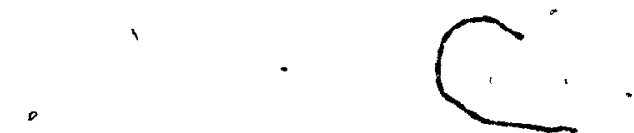

An array of three $147.3 \mathrm{~cm} \times 25.4 \mathrm{~cm}$ counters, VEU, covered the east side of the apparatus at the same location along the beam axis as the VX counters. The logical of of the signals from these counters was combined with the logical OR of signals from an identical array of counters, VED, $3.1 \mathrm{~m}$ downstream but at the same position relative to the beam axis. A VW signal was formed from the outputs of two identical arrays, VWU and VWD, on the west side of the apparatus.

A HALO signal was defined as the logical. OR of the VI, VE and VW signals indicating that a particle had passed through both of the innner arrays or either pair of the outer arrays on opposite sides of the apparatus. The HALO signal was used to veto the BEAM signal so that beam particles accompanied by a halo muon were ignored by the apparatus. Typically 4 percent of the beam particles were vetoed, but the trigger rate was reduced by a factor of 4 , depending on the beam tune.

\subsection{Target}

.

Data was taken with three different nuclear targets, tungsten, copper and beryllium, to investigate the $A$ dependence of the cross section. Initially all targets were chosen to be 1.0 absorption lengths for incident. antiprotons, and were machined as $15.24 \mathrm{~cm}$ diameter cylinders. A 1.5 interaction length tungsten target was used during the 1982 run. A 0.4 interaction length. tungsten target was also used for a fraction of the 1982 
run to estimate the effects of reinteraction. The physical lengths and densities of all the targets used are. listed in Table 2. The 1.0 interaction length targets were each divided into two segments and separated in such a way as to minimize any difference in acceptance between the various nuclear lements. Counters were placed between the segments to idenafy. In which segment the interaction took place as an aid to the reconstruction programmes. The three segmented nuclear targets were mounted on a remote mantpulator and interchanged regularly during the run to minimize any possible systematic effects.

The bulk of the data was taken with the tungsten target, however, to maximize the overall event rate.

\subsection{Target Counters}

The target counters consisted of $15.24 \mathrm{~cm}$ diameter disks of $0.635 \mathrm{~cm}$ thick plastic scintillator coupled to Amperex 56AVP photomultipliers [63] with $60 \mathrm{~cm}$ long lucite light guides. As with the beam and Cerenkov counters, the signals from the target counter photomultipliers were clipped and damped in an attempt to achieve the highest possible counting rate consistent with the conflicting requirements of high gain and wide dynamic range. The counters were mounted at the base of the target manipulator with the light guide holding the scintillator in the beam Iine so as not to ' interfere with the movement of the targets. One counter, T6, was mounted between the two segments and a second, T7, was mounted in the gap between the downstream target segment and the face of the absorber. Signal amplitudes from the target counters were recorded by Lecroy $2249 \mathrm{~A} 12$ channel $A D C$ 's[66] for use by the reconstruction programmes. 


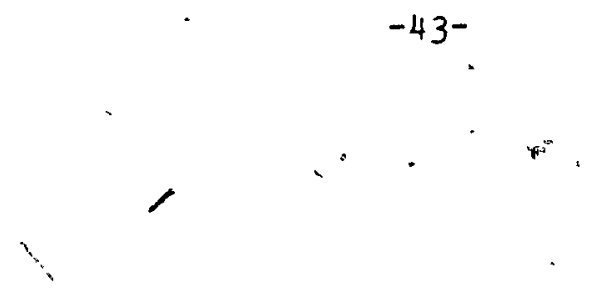

Table 2 - Target Densities and Lengths

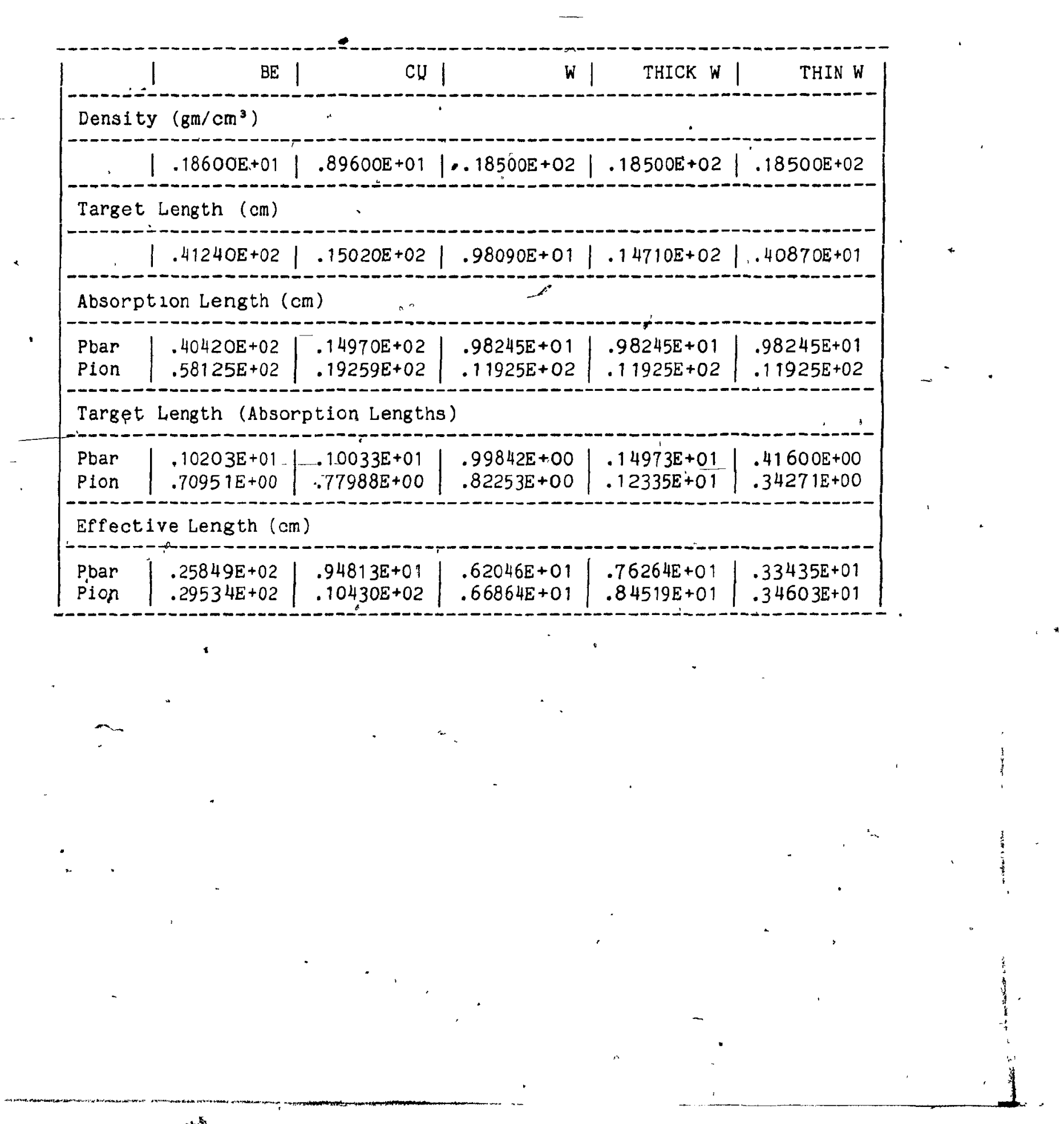




\subsection{Beam Dump}

Hadrons from interactions in the target and beam particles that did not interact were absorbed by a beam dump downstream of the target. A copper core of twelve $12.7 \mathrm{~cm}$ thick slabs covered the full acceptance of the spectrometer, giving a total thickness of 8 absorption lengths for $125 \mathrm{GeV} / \mathrm{c}$ pions. Copper was chosen for its relatively high density and acceptable ratio of radiation length to absorption length. Steel shlelding on either side of the copper absorber minimized leakage from the sides and helped to attenuate of $f$ axis beam halo.

\subsection{Absorber Chamber}

Two plane of proportional wires were located approximately two thirds of the way fhrough the dump to aid in the vertex reconstruction. The chamber package included 3 signal planes ( $X, U$ and $V$ ), 4 cathode planes, and 2 ground planos sharing the same gas enclosure. Only the $U$ and the $V$ planes could be instrumented because of space constraints. Signal planes consisted of 480 tungsten wires of $15 \mu$ diameter, spaced so that adjacent wires were separated by $1.5 \mathrm{~mm}$. In the $\mathrm{X}$ direction. Cathode planes were wound of $63 \mu$ diameter tungsten wire on $a^{\prime} 0.5 \mathrm{~mm}$ spacing. The wires in the $U$ and $V$ planes were rotated from the vertical ( $Y$ axis) by \pm 16.7 degrees. The readout electronics. were identical to those.used with the beam telescope, and the chamber used the same gas mixture. 


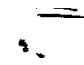

$$
-45-
$$

\subsection{Absorber Counter}

A four element scintillation counter immediately downstream of the dump detected muons produced at angles greater than $70 \mathrm{mr}$ with respect to the nominal beam direction. Because the mass of a muon pair depends linearly on the opening angle in the laboratory frame, this counter provided a high mass blas when used in one of the fast triggers. Four quadrants (ABUL, ABUR, $A B D L$ and $A B D R$ ) made of $0.9525 \mathrm{~cm}$ thick PILOT A plastic scintillator[68] covered the full acceptance of the spectrometer except for a $0.305 \mathrm{~m}$ diameter hole centred on the beam axis. Figure 12 shows the counter and the shape of two of the four identical quadrants. Adjacent quadrants extended beyond the centre 1 ine by $12.7 \mathrm{~cm}$ ' in $X$ and by $7.62 \mathrm{~cm}$ in $Y$ to cover the full acceptance of the corresponding rear muon counter quadrant.

Each quadrant was coupled to an Amperex 56AVP photomultiplter [63] connected to a resistive divider base. As with the beam hodoscopes, the last two dynode stages were clamped with zener diodes and the output signals. were clipped with a 3 ns stub of RG174 50 a coaxial cable terminated with a $25 \Omega$ resistor. The photomultipliers were shlelded from the fringe field of , the analysis magnet by, $1 \mathrm{~cm}$ wall thickness steel pipe, and enclosed in a $1 \mathrm{~cm}$ thick steel box. Discriminated signals from the four quadrants were used in one of the fast triggers and recorded for use in the analysis.

3.12 Drift Chambers

$$
x^{2}-4
$$

Muon trajectories in the spectrometer were measured by 18 planes of wire drift chambers. The design and operation of drift chambers have been 

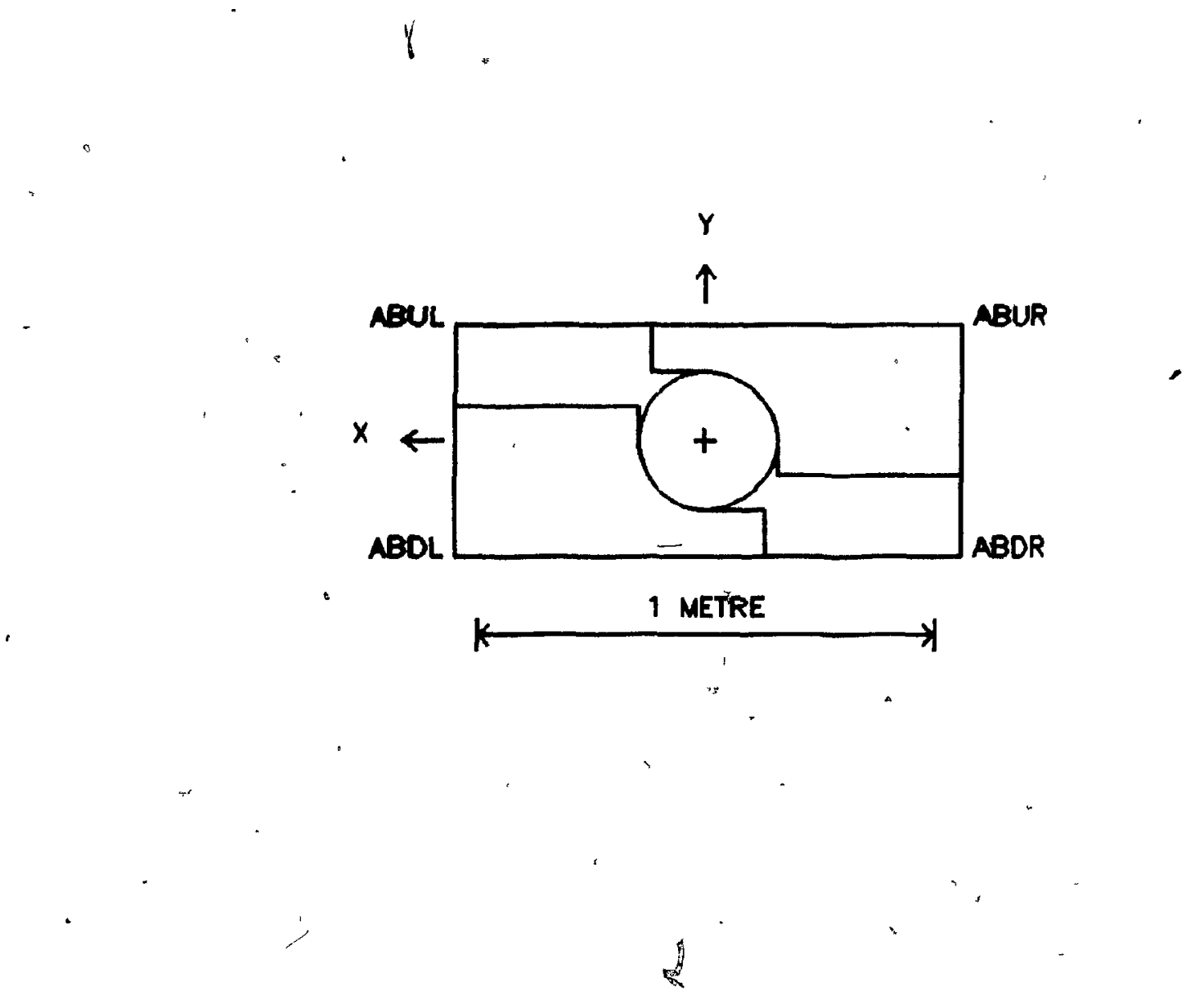

Figure 12 - Absorber Counter

The absorber counter was used to detect:muons produced at large angles to the beam. Two of the four identical elements of the counter are shown in outline. Only the actual sointillator is shown. 
reviewed extensively[69][70][71] and w1ll not be discussed in detail here. The nine planes in front of the analysis magnet were grouped Into three chambers of three planes each, as were the nine planes following the magnet. Wire spacings, diameters and orientations are listed in Table 3 and Table 4. The details of each chamber varied because they were constructed at different institutions.

The first chamber consisted of three signal planes wound on glass fibre-epoxy frames and sharing common cathodes. The cathode planes were also wound on glass-epoxy frames. The outermost cathodes were shielded by ground planes' of similar construction. The glass-efoxy frames were bolted to an aluminum support frame and sealed with a noncorrosive silicon rubber. The cathodes were run at negative high voltage and the anode wires were direct coupled to the amplifiers.

The second and third chambers shared a common gas enclosure. The signal wires for these chambers were supported by precision inserts set in a jig bored aluminum frame. The cathodes, of stretched aluminum foll, were glued to an aluminum frame and were run at ground potential. Anode wires were capacitively coupled to the amplifiers. The physical construction of the fourth chamber was identical to that of the second and third chambers.

The final two chambers were similar to the first chamber in that wound signal planes and cathodes were used. Each plane was wound on a separate self supporting glass-epoxy frame. The cathode planes were run at negative high voltage and the signal wires were again connected directly to the amplifiers. The frames of each chamber were contained in a single gas - "enclosure with mylar windows. 
Table 3 - Drift Chamber Parameters I

\begin{tabular}{|c|c|c|c|c|c|c|}
\hline Chamber & $\begin{array}{l}\text { Cell } \\
\text { Size } \\
(\mathrm{cm})\end{array}$ & $\begin{array}{l}\text { Signal } \\
\text { Wire } \\
\text { Diameter } \\
\text { (cm) }\end{array}$ & $\begin{array}{l}\text { Cathode } \\
\text { Plane } \\
\text { Diameter } \\
(\mathrm{cm})\end{array}$ & $\begin{array}{l}\text { Drift } \\
\text { Wire } \\
\text { Diameter } \\
(\mathrm{cm})\end{array}$ & $\begin{array}{l}\text { Effective } \\
\text { Aperture } \\
\text { (cm) }\end{array}$ & $\begin{array}{l}\text { Cathode } \\
\text { Potential }\end{array}$ \\
\hline $\mathrm{DC} 1$ & 0.6 & $\begin{array}{l}2.0 E-3 \\
\text { Tungsten }\end{array}$ & $\begin{array}{l}6.35 E-3 \\
\text { Cu-Be } \\
0.1016 \\
\text { Pitch }\end{array}$ & $\begin{array}{l}6.35 E-3 \\
\mathrm{Cu}-\mathrm{Be}\end{array}$ & $50 \times 100$ & $-H . V$. \\
\hline DC2 & 1.27 & $\begin{array}{l}2.54 \mathrm{E}-3 \\
\text { Tungsten }\end{array}$ & $\begin{array}{l}2.54 \mathrm{E}-3 \\
\mathrm{~A} 1 \text { Foil }\end{array}$ & $\begin{array}{l}0.0127 \\
\mathrm{Cu}-\mathrm{Be}\end{array}$ & $50 \times 100$ & Ground \\
\hline DC3 & 1.27 & $\begin{array}{l}2.54 \mathrm{E}-3 \\
\text { Tungsten }\end{array}$ & $\begin{array}{l}2.54 \mathrm{E}-3 \\
\text { Al Foil }\end{array}$ & $\begin{array}{l}0.0127 \\
\mathrm{Cu}-\mathrm{Be}\end{array}$ & $50 \times 100$ & Ground \\
\hline DC4 & 1.905 & $\begin{array}{l}2.54 \mathrm{E}-3 \\
\text { Tungsten }\end{array}$ & $\begin{array}{l}2.54 \mathrm{E}-3 \\
\text { AI Foil }\end{array}$ & $\begin{array}{l}0.0127 \\
\mathrm{Cu}-\mathrm{Be}\end{array}$ & $100 \times 200$ & Ground \\
\hline DC5 & 1.905 & $\begin{array}{c}2.54 E-3 \\
\text { Tungsten } \\
.\end{array}$ & $\begin{array}{l}6.35 E-3 \\
\text { Cu-Be } \\
0.1778 \\
\text { Pitch }\end{array}$ & $\begin{array}{l}6.35 E-3 \\
\text { Cu-Be }\end{array}$ & $167 \times 335$ & $\begin{array}{c}-\mathrm{H} . \mathrm{V} \cdot \\
\cdot \quad \\
\end{array}$ \\
\hline DC6 & 1.905 & $\begin{array}{l}2.54 E-3 \\
\text { Tungsten }\end{array}$ & $\begin{array}{l}6.35 E-3 \\
\text { Cu-Be } \\
0.1778 \\
\text { Pitch } \\
\quad .\end{array}$ & $\begin{array}{l}6.35 E-3 \\
C u-B e\end{array}$ & $167 \times 335$ & $-H . V$. \\
\hline
\end{tabular}


ᄂ

Table 4 - Drift Chamber Parameters II

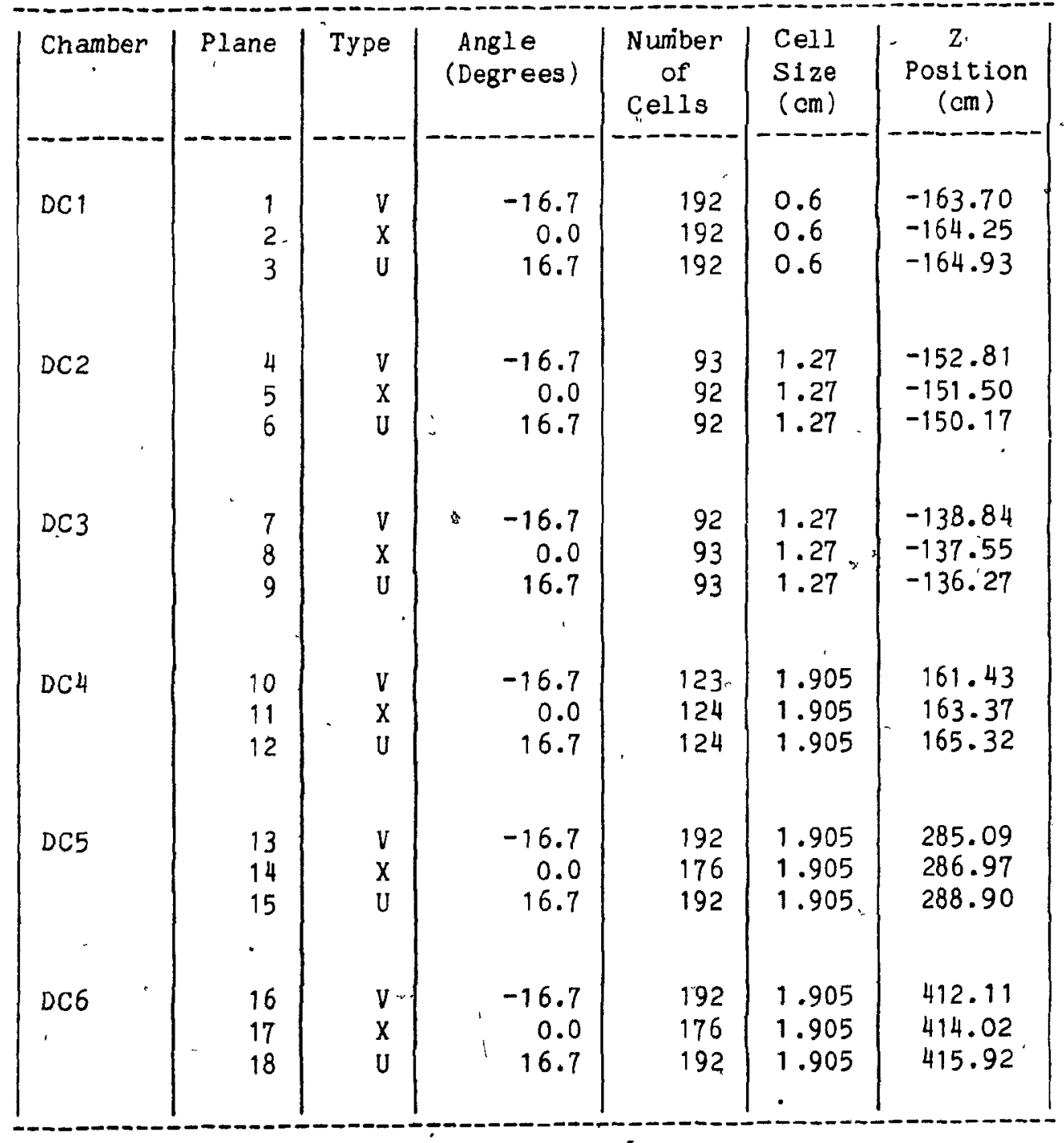


All chambers used a gas mixture of 50 percent argon and 50 percent. ethane to achieve a low saturation voltage in the rear chambers, while providing a high drift velocity $(50 \mu / \mathrm{ns})[72]$ and minimal dead time in the front chambers. Flow rates were set to flush the individual chamber volumes once every two days.

The signal wires were connected to Lecroy MVL100 monolithic amplifier-discriminator chips[66] consisting of a $100 \times$ gain amplifier followed by a voltage programmable ECL comparator. The control voltage was set to give discriminator thresholds between $80 \mathrm{mV}$ and $130 \mathrm{mV}$ at the signal, wires depending on the chamber. The differential ECL outputs of the MVL $100^{\prime}$ s were connected to Lecroy 2770A drift chamber digitizers[66] and read out via CAMAC[73]. Each digitizer contained 96 time to digital. converters (TDC's) with a full scale time range of approximately 256 ns for 256 counts. To correct for a channel to channel variation of 10 percent and ensure an accurate measurement of the drift time, the TDC's were calibrated at the beginning of each data tape.

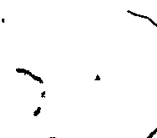

The calibration system applied a series of 5 rolt pulses to the field shaping wires of the chambers under computer control. These pulses induced signals on the sense wires, causing the discriminators to trigger and sending start pulses to the' TDC's. A common stop pulse was sent to the TDC's after a computer controlled delay. A series of 600 calibration events was written automatically at the beginning of each data tape. Ten pulses at 10 different delay times were" sent to each of the six chambers in turn. Prior to the analysis of the data tapes, the calibration events were read and straight lines were fit to the number of counts vs delay curves to obtain a set of calibration constants for each digitizing channel on each 


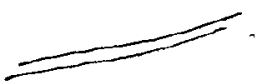

tape. After.calibration the TDC's had a time 'resolution of better than 1 ns.

A stand alone version of the calibration system was used to monitor the drift chamber electronics and diagnose malfunctioning channels throughout the data taking.

3.13 Análysis Magnet

The spectrometer magnet was a-large window frame dipole with saddle colls containing 240 turns. The magnet aperture measured 90 cm vertically and $180 \mathrm{~cm}$ horizontally with a $75 \mathrm{~cm}$ long iron yoke. At the nominal setting of 2400 amperes, the main component $t_{i}$ of the field gave a horizontal momentum change to the particles of $830 \mathrm{MeV} / \mathrm{c}$. The field was mapped by measuring the current induced in 3 orthogonal colls as they moved through the magnetic field under computer control. NMR measurements of the field at the centre of the magnet were used for absolute calibration. The magnet current was monitored continuously by a precision shunt, in addition. to the standard power supply current transducer. The field was monitored using a Hall probe fixed to the lower pole face of the magnet. The agreement between, the current shunt and the Hall probe was always better than 0.1 percent. Using the measured $\int B \cdot d l$ of the magnet, the $\psi$ mass was reproduced to better than 1 percent accuracy. The polarity of the field was reversed"periodically during the course of the 1981 run to check for systematic effects associated with properites of the beam halo. 
3.14 Charged Particle Hodoscope

Two scintillation counter hodoscopes, $\mathrm{CPX}$ and CPY, located immediately $\therefore$ behind the final drift chamber measured the $X$ and $Y$ positions of charged tracks respectively.

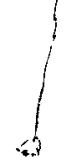

The CPX hodoscope consisted of two rows of counters made of $1 \mathrm{~cm}$ thick $\times 4 \mathrm{~cm}$ wide $\times 1 \mathrm{~m}$ long NE110 plastic scintillator [62], and arranged as shown in Figure 13. The counters were coupled tofEMI 9807B, and 9814B photomultiplier tubes[74] with transistorized bases[65]. A simplified schematic diagram of the CPX electronic logic is'shown in Figure 14." The. output signal from each photomultiplier on the top row of 92 counters was summed with the signal from the corresponding counter in the lower 'row before discrimination. Logical signals from the combined CPX counters were summed and rediscriminated to supply a $C P X \geq 2$ signal to the fast trigger logic when more than two of the counters were hit. The discriminated signals from all of the CPX counters were recorded for each event.

The CPY hodoscope consisted of $481 \mathrm{~cm}$ thick $\times 8 \mathrm{~cm}$ wide $\times 2 \mathrm{~m}$ long NE110 plastic scintillation[62] counters coupled to Amperex 56AVP[63] photomultiplier tubes with transistorized bases[65]. The counters were arranged in two vertical columns of 24 counters each extending from $x=0.0$ to $X= \pm 2.0 \mathrm{~m}$. Logical signals from the CPY counters were used by the trigger processor. As with the CPX counters, discriminated signals from the CPY countèrs were recorded for each event.

Central counters in both hodoscopes were retracted to leave a $32 \mathrm{~cm}$ square beam hole. 

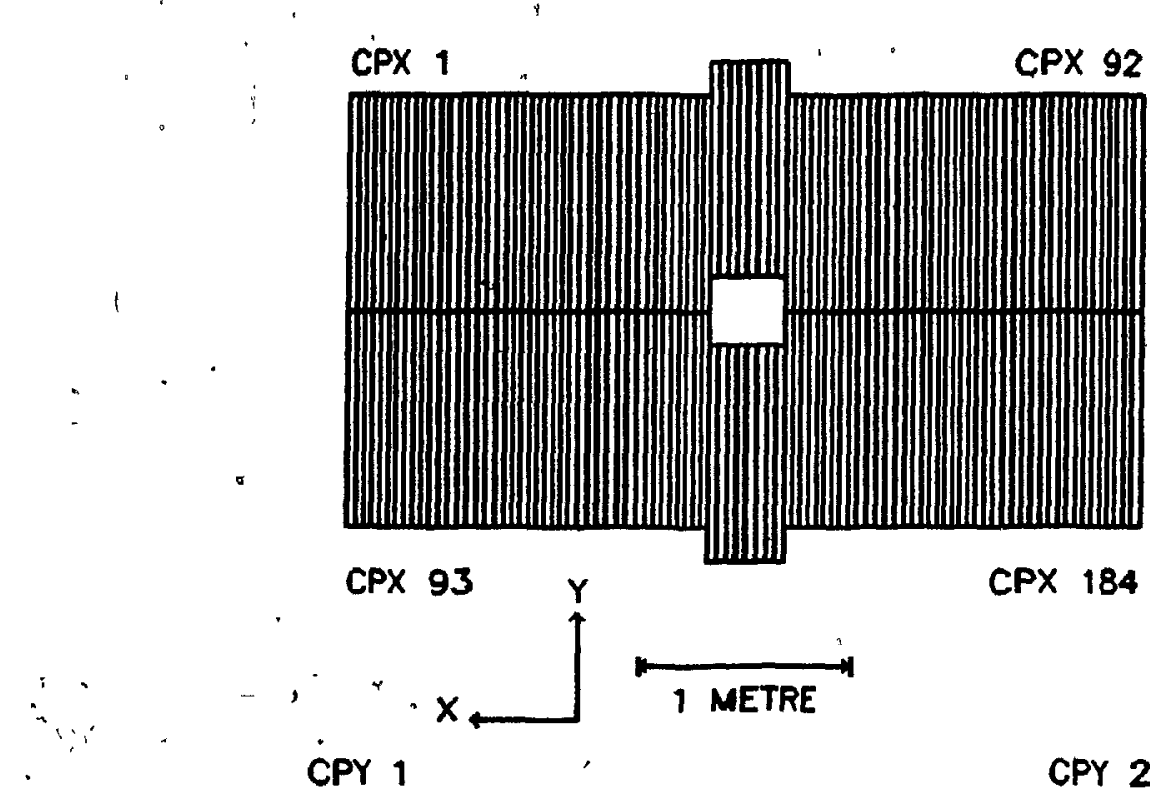

CPX 184

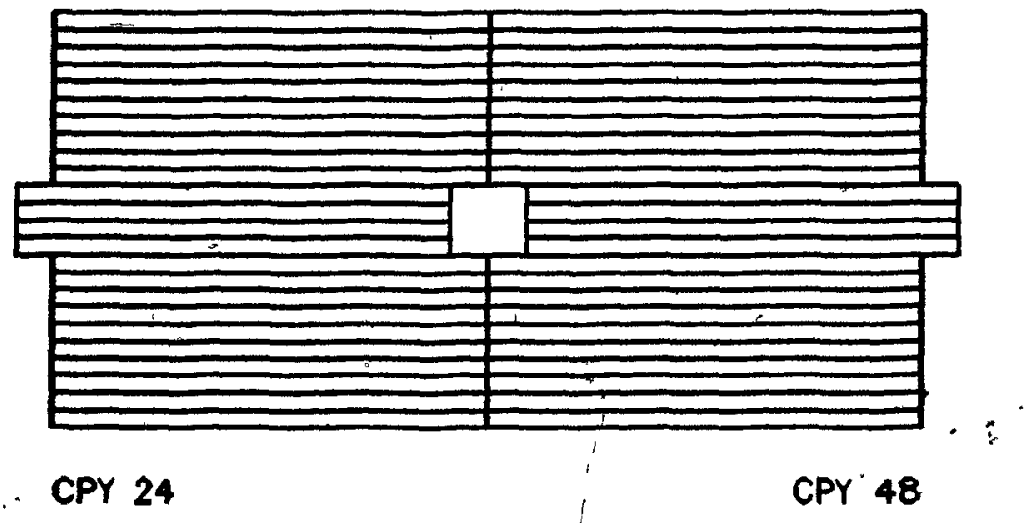

Eigure 13 - Charged Particle Hodoscope

The Charged Particle Hodoscopes, CPX and CPY, measured the $X$ and $Y$ positions of charged tracks as discussed in the text. The counters are shown looking along the beam direction and the scale is indicated.

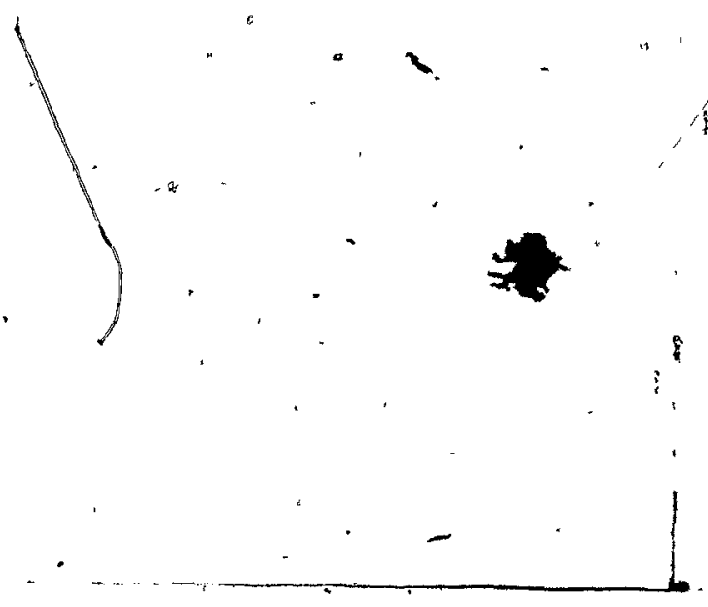




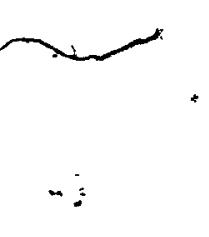

s.

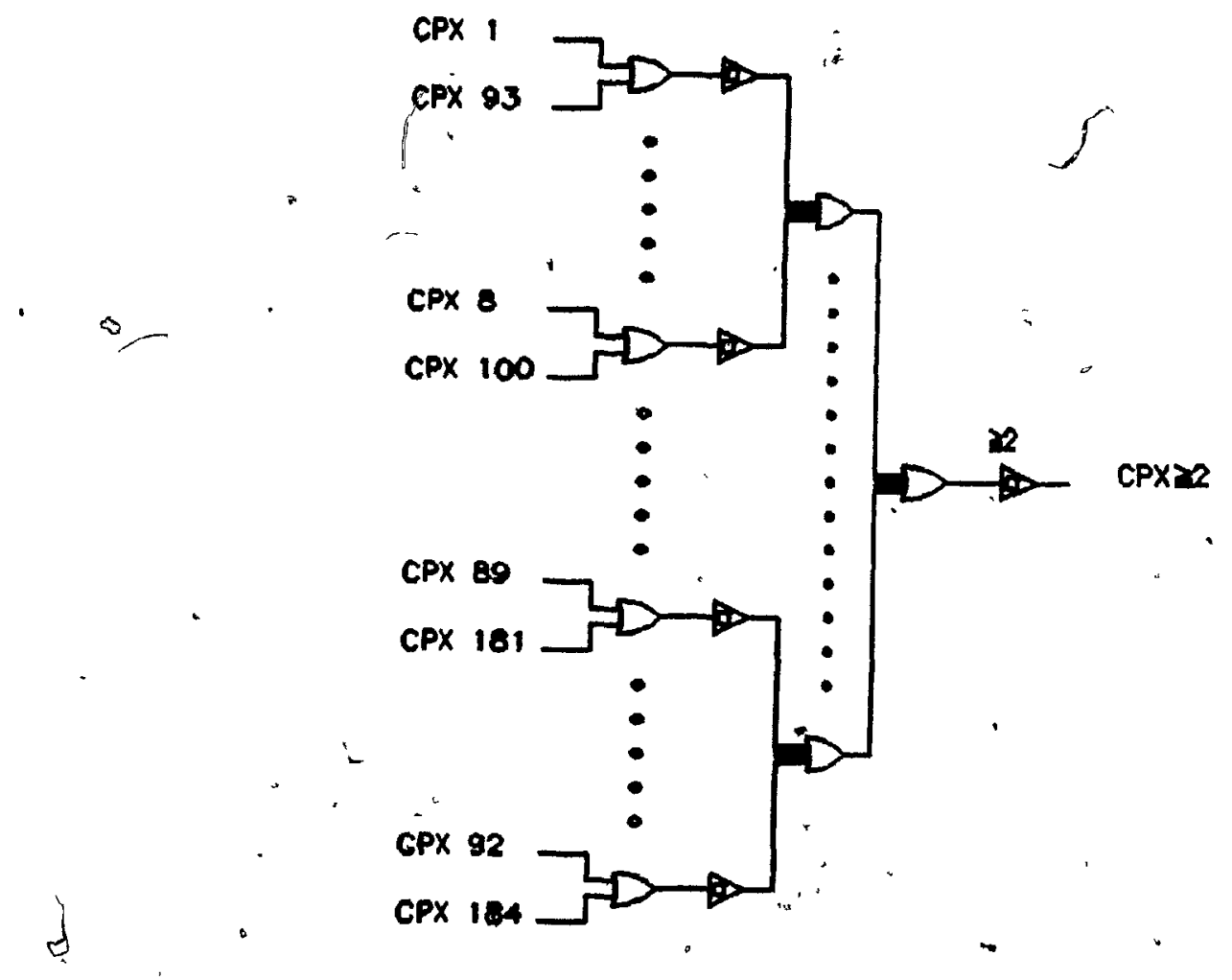

Figure 14 - Charged Particle Hodoscope Logic

This simplified schematic diagram shows the electronic logic used to define the CPX $\geq 2$ signal fromt the outputs of the CPX counters. Photomultiplier signals from the upper row of counters were summed with signals from the corresponding counters in the lower row prior to discrimination. The linear sum of the discriminated signals was rediscriminated to define the CPX $\geq 2$ signal. 
3.15 Muon Hodoscopes

Muons were identified by requiring them to traverse a telescope of three scintillation counter hodoscopes interleaved with walls of steel and concrete. The arrangement of the counters in the first plane and a plan view of the three planes is shown in Figure 15 .

The first muon hododscope, $\mu 1$, consisted of two rows of 30 $1 \mathrm{~cm}$ thick $\times 20.3 \mathrm{~cm}$, wide $\times 1.45 \mathrm{~m}$ long counters made of NE114 plastic scintillator[62] located behind - a $1.106 \mathrm{~m}$ thick steel wall. The four central counters in the first plane were retracted to leave a $20.32 \mathrm{~cm}$ square beam hole. A second hodoscope, $\mu 2$, with two rows each consisting of $311 \mathrm{~cm}$ thick $\times 23 \mathrm{~cm}$ wide $\times 1.57 \mathrm{~m}$ long counters followed a $61 \mathrm{~cm}$ thick steel wall. A third hodoscope,,$\mu 3$, with 62 $1 \mathrm{~cm}$ thick $\times 26.7 \mathrm{~cm}$ wide $\times 1.75 \mathrm{~m}$ long counters arranged in two rows of 31 counters each, followed a $90 \mathrm{~cm}$ thick concrete wall. Counters in the first plane were spaced $20.574 \mathrm{~cm}$ centre to centre so that there was no overlap between adjacent counters, and the array was positioned symmetricalip about the beam axis. Counters' in the second and third planeswere spacied $22.225 \mathrm{~cm}$ and $24.57 \mathrm{~cm}$ apart respectively.

All of the muon counters were coupled to Amperex 2212B photomultipliers[63] with transistorized bases[65]. Discriminated signals from counters in the upper row of the first wall were combined with the logical $O R$ of pairs of counters in the upper rows of the second and third planes to define muon triple coincidence signals TC1-TC30 as follows:

$T C 1=\mu 1-1 \cdots(\mu 2-1+\mu 2-2)-(\mu 3-1+\mu 3-2)$,

$T C 2=\mu 1-2 \cdot(\mu 2-2+\mu 2-3) \cdot(\mu 3-2+\mu 3-3)$, 

and

$$
\mathrm{TC} 30=\mu 1-30 \cdot(\mu 2-30+\mu 2-31) \cdot(\mu 3-30+\mu 3-31)
$$

as shown in Figure 16. The signals TC31-TC60 were' defined for the lower row of counters as follows:

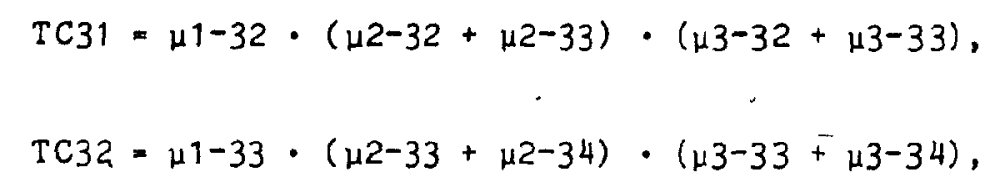

and

$$
\mathrm{TC60}=\mu 1-61 \cdot(\mu 2-61+\mu 2-62) \cdot(\mu 3-61+\mu 3-62) .
$$

To suppress coincidences caused by halo muons, the counters of each triple coincidence channel were centred to line up along the path taken by an inf inite momentum muon produced in the target. The widths of the counters in the second and third planes were chosen to give good acceptance down to muon momenta of $6 \mathrm{GeV} / \mathrm{c}$ when allowances were made for multiple scattering.

Logic signals from all of the counters were recorded and read out via CAMAC[73] for each event. The time elapsed between the event trigger and the signals from the first plane of muon counters was measured and recorded $\because$ using Lecroy 2249A 8 channel TDC's[66] so that backgound due to beam halo or hadronic decays could be studied of 1 line.

3.16 Fast Logic

Standard NIM[67] logic modules were ysed to form three fast trigger - . signals if appropriate combinations of counters had Qutputs consistent with the signature of a high mass muon pair, a single beam particle hititing the 


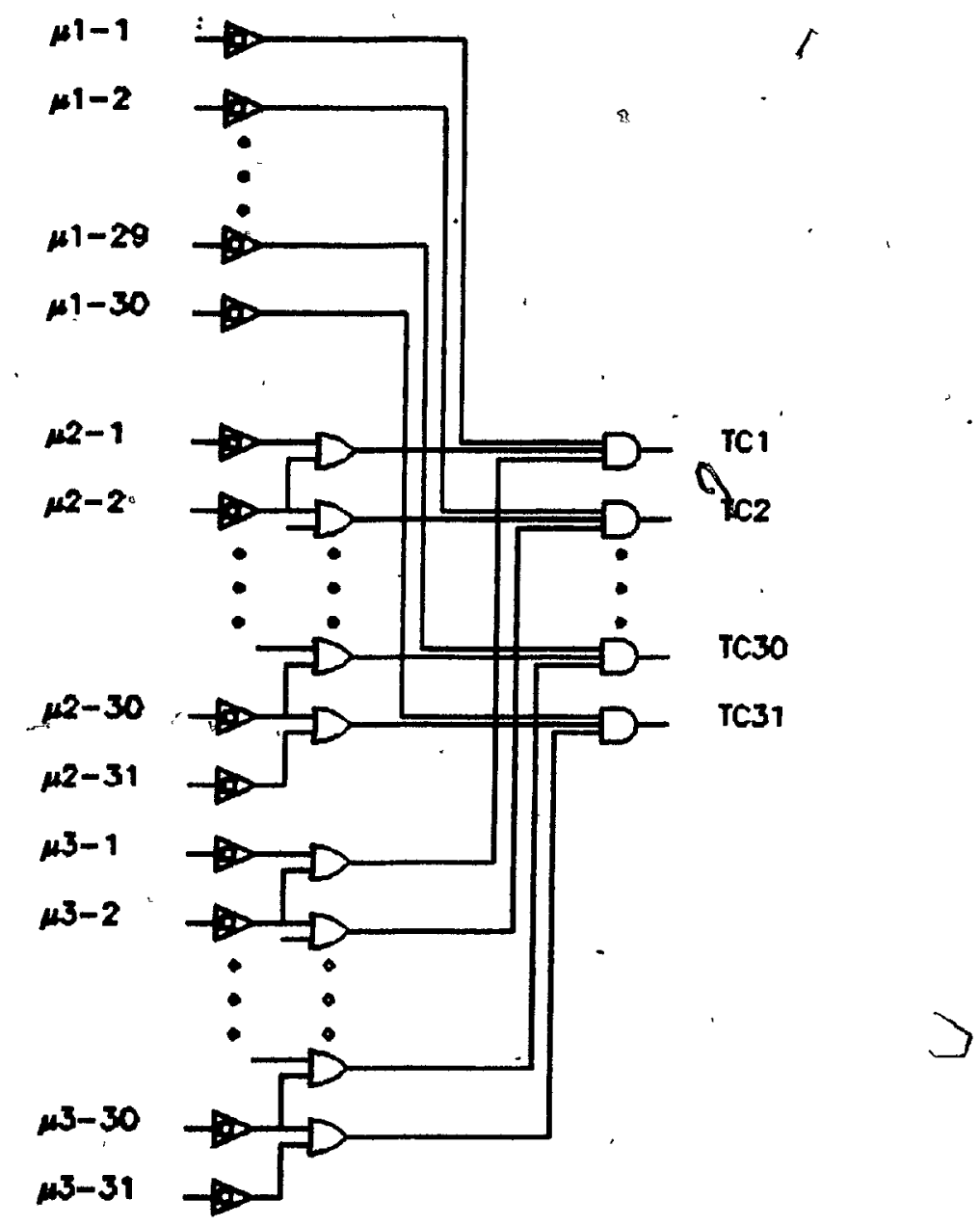

Figure 16 - Muon Triple Coincidence Logic

This simplified schematic diagram shows the electronic loglc used to define the muon triple coincidence signals TC1-TC30 from the photomultipler outputs of counters in the top rows of the three muon hodoscopes as discussed in the text. Identical logic "was used to define the signals TC31-TC60 from the outputs of counters in the lower rows. 
target and two muons traversing the apparatus. A simplifled schematic diagram of the fast.trigger logic is shown in Figure 17. In the absence of any transverse momentum, muons from a high mass pair would enter the apparatus in different geometrical quadrants. The accidental coincidence between a beam halo muon and a muon produced by hadronic decay would not be so constrained. The four fold symmetry of the apparatus about the bean axis was exploited in the definition of the trigger signals to bias against muon pairs produced by such accidental coincidences.

A muon was assumed to have traversed the apparatus if one of the muon triple colncidences produced a signal. The $60 \mathrm{TC}$ channels were combined together 15 at a time to form 4 quadrant signals as follows:

$$
\text { HQUAD1 }=\mathrm{TC} 1+\mathrm{TC2}+\ldots+\mathrm{TC} 15
$$

HQUAD2 $=\mathrm{TC} 16+\mathrm{TC} 17+\ldots+\mathrm{TC} 30$,

LQUAD3 $=\mathrm{TC} 31+\mathrm{TC32}+\ldots+\mathrm{TC} 45$,

and

HQUAD3 $=\mathrm{TC46}+\mathrm{TC47}+\ldots+\mathrm{TC60}$.

A QUAD signal was defined as

$$
\begin{aligned}
\text { QUAD }= & (\mu Q U A D 1 \cdot \mu Q U A D 2)+(\mu Q U A D 1 \cdot \mu Q U A D 3)+ \\
& (\mu Q U A D 1 \cdot \mu Q U A D 4)+(\mu Q U A D 2 \cdot \mu Q U A D 3)+ \\
& (\mu Q U A D 2 \cdot \mu Q U A D 4)+(\mu Q U A D 3 \cdot \mu Q U A D 4),
\end{aligned}
$$

Indicating that two muons had passed through different quadrants of the apparatus. The timing of the QUAD signal was set by the leading edge of the $\pi^{-} / \overline{\mathrm{p}}$ signal from the beam tagging logic.

The lowest level fast trigger was defined as
TRIGO = $\left(\pi-J_{\bar{p}}\right) \cdot$ QUAD. $\therefore$ 


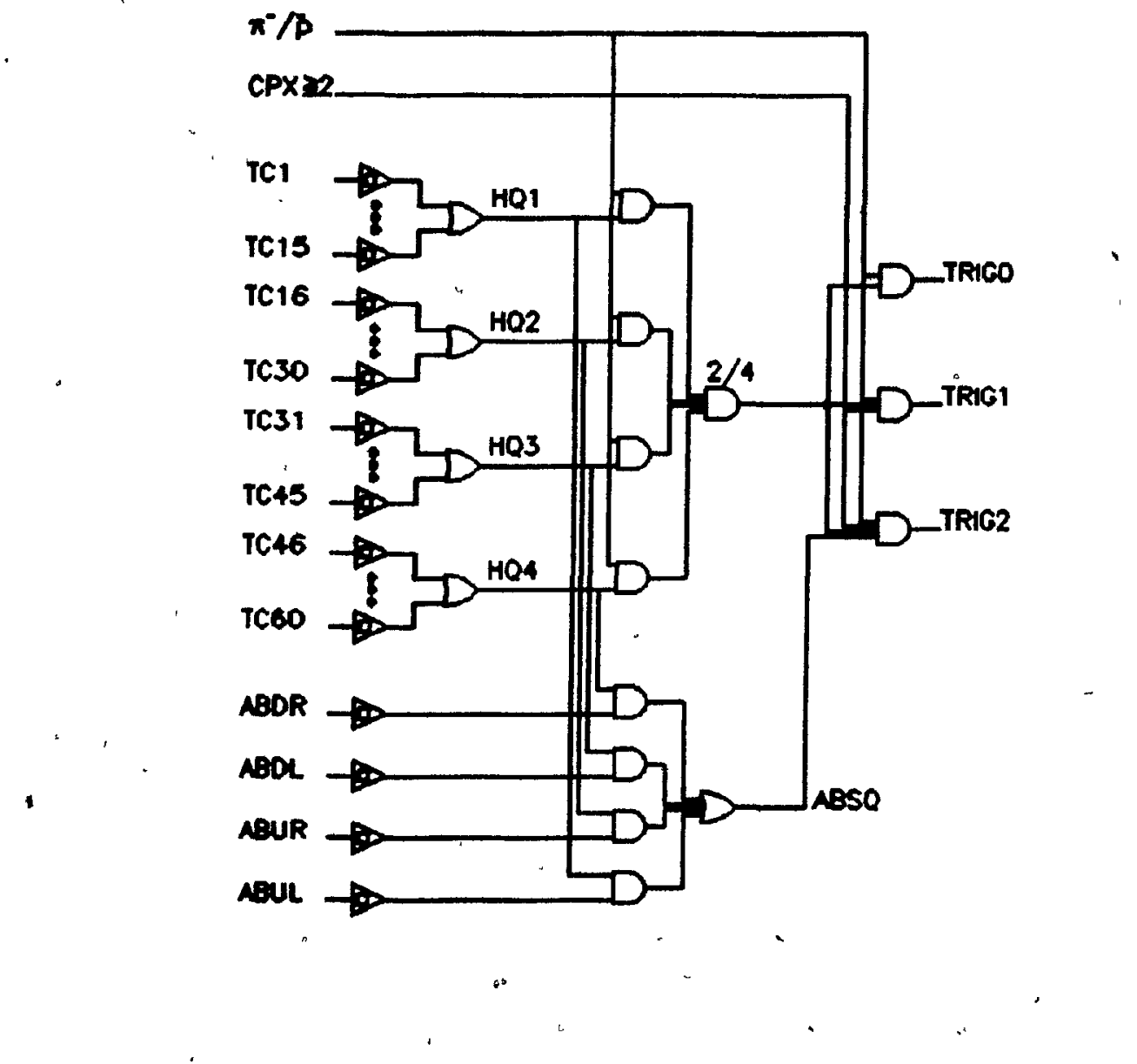

Figure 17 - Trigger Logio

This simplifed, schematic diagram shows the electronic logic used to define the three fast trigger signals. These signals were separately prescaled and then combined with P'ION and PBAR signals from the beam logic to give a inal trigger signal: 

detail elsewhere[75], and therefore only the algorithm used and its implementation will be outlined here.

Wires hit in the $X$ planes of the chambers downstream of the magnet, CPX counter outputs, CPY counter outputs, and muon triple coincidence channel outputs were stored by a fast ECL encoder gated by the trigger logic. A hard wired trigger processor employing the Fermilab ECL-CAMAC system of modules[76][77] calculated the invariant mass of all possible muon pairs and rejected events which reconstructed to a mass of less than $2.0 \mathrm{GeV} / \mathrm{c}^{2}$.

The processor examined ali, combinations of drift chamber hits and - counter outputs to find candidate tracks in the $X$ projection. The momenta and opening angles in this projection were calculated assuming that the tracks originated in the target. The CPY counter information was used to give an upper bound on the opening angle in the $Y$ projection. The invarlant mass was calculated for each pair of tracks using the approximation ,

$$
M^{2}=p_{1} p_{j} \theta_{i j}^{2}
$$

where $\dot{p}_{1}$ and $p_{j}$ are the momenta of the $i^{\text {th }}$ and $j^{\text {th }}$ tracks respectively, and $\theta_{i j}$ is the angle between the tracks in radians. Events with any combination giving a mass above $2.0 \mathrm{GeV} / \mathrm{C}^{2}$ were read out via CAMAC[73] and recorded on magnetic tape. Events with no candidates reconstructing to at least the minimum mass were cleared and reset without being read.

The processor made extensive use of precalculated tables stored in $\mathrm{f}$ ast memories to simplify the logic and improve the performance. Typically about 5 us were required to evaluate an event, and events complicated enough to require more than 100 microseconds to evaluate were accepted unconditionally. 
During the development, of the processor, a diagnostic and simulation programme was written using the interactive language FORTH[78]. Test events from the simulation programme and data tapes allowed the processor to be tested in stages by comparing internal values to results calculated by the simulator.

A fraction of the triggers obtained during any given run were recorded unconditionally, so that the trigger processor performance and efficiency could be checked. The usefulness of the trigger processor can be seen immediately from Figure 18, which shows the large number of low mass events that were rejected. Its ficiency can be seen from Figure 19, by comparing the number of events rejected by the processor to the results of a full reconstruction. The processor reduced the overall trigger rate by a factor of 5-10, depending on the beam tune, with an efficiency of better than 99 percent.

3.18 Data Acquisition

The experiment was controlled and montored using a PDP 11/45 computer[79] running ${ }^{\circ}$ under the RSX11/M operating system. Data recording modules were connected to the computer using 3 parallel CAMAC[73], branches. Two 800 BPI magnetic tape drives were used to record the events for of reconstruction.

One CAMAC branch was used to service the trigger processor described in the last section. A second branch contained the following: 


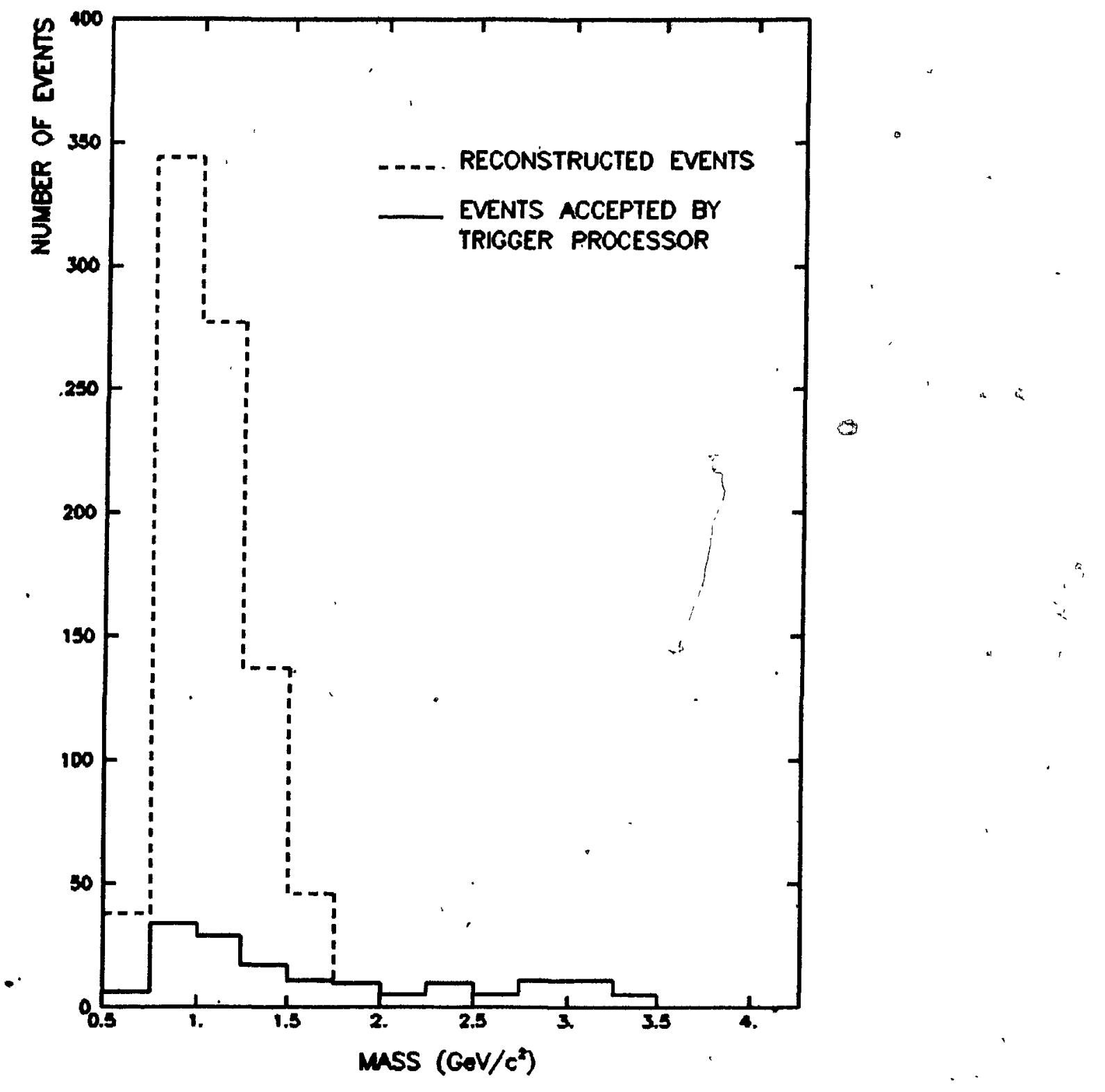

Figure 18 - Trigger Processor Performañ̄e

The dashed line shows the mass spectrum of muon pairs reconstructed of 1 ine from events where the processor information was not used in the trigger. The solid line shows the mass spectrum of the events accepted by the trigger processor. The large numbers of low mass events rejected by the trigger processor resulted in a substantial reduction of the trigger rate. 


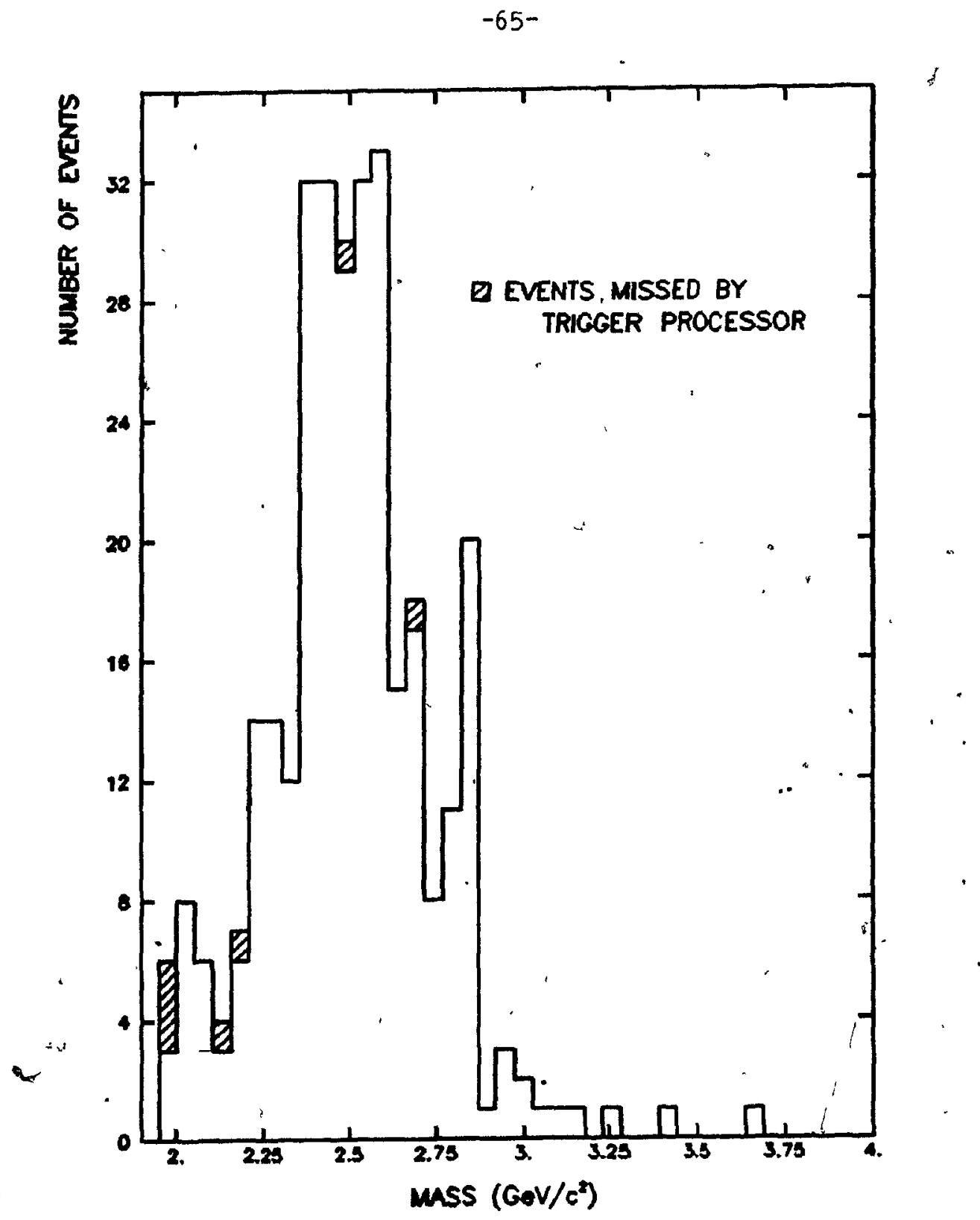

Figure 19 - Trigger Processor Efficiency

This histogram shows the mass spectrum of muon pairs reconstructed offline from events where the processor information was not used in the trigger. The hatched areas show events that would have been rejected by the trigger processor. The measured efficiency of the processor was 0.99 . 
1. scalers used to monitor counting rates at various stages of the fast trigger logic and to record beam fílux totals;

2. analog to digital converters (ADC's) used to monitor the
performance of various counters throughout the experiment;

3. time to digital converters (TDC's) used to monitor the Cerenkov counters and the first plane of muon counters; and

4. coincidence registers which were used to record the state of all the counters in the experiment at the time of the trigger.

A third branch was used to read out the wires hit in the proportional and drift chamber systems.

The output of the trigger processor was used to interrupt the computer, which then read out the three CAMAC branches using a Fermilab supplied data acquistion package[80][81]. The events were transferred directly into a 256 Kbyte bulk memory[82] during the spill, and transferred to magnetic tape at the end of each spill. The" information recorded for each event included the following:

1. the date and time of the splil;

2. a list of all proportional chamber wires hit;

3. a list of all drift chamber wires hit and the drift times;

4. a complete list of all counters hit in coincidence with the fast 
trigger; and.

5. $A D C$ and $T D C$ information for monitioring purposes. In addition at the end of each spill, a list of scaler sums was written to the tape. This Ist included the following:

1. antiproton and pion flux totals;

2. primary beam intensities;

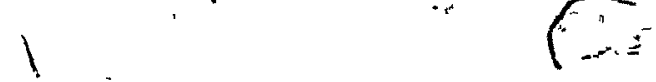

3. counting rates in the Cerenkov counters and the beam telescope;

4. counting rates of the absorber counters and selected muon counters; and

7

-5. counting rates at various stages in the beam $\log 1 \mathrm{c}$ and the fast trigger logic.

The reconstruction of the muon pair kinematic variables from the information on the data tapes will be discussed in the next chapter.

\subsection{Online Analysis}

1

During the data taking the spectrameter performance was monitored online by" several analysis programmes based on SUPERGRAM[83][84], a histogramming package written at the University of Michigan. By efficiently using disk storage, SUPERGRAM was capable of accessing over $10^{5}$ bins of 
'Information while requiring only $6 \times 10^{3}$ words of programme memory space. The online analysis perforged the following functions:

1. kept track of scaler sums to give up to date beam totals, and to monitor the performance of the beam tagging. system and the trigger;

2. histogramed wire and time distributions for the drift chambers and proportianal chambers to aid in the diagnosis of malfunctioning channels and to monit or chamber efficiencies.

3. histogrammed TDC, ADC and latch information to monitor counter and trigger performance;

4. controlled the data acquistioñ system, beginning and ending runs; and

5. plotted histograms under console command.

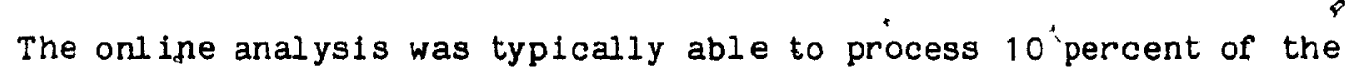
events in the time between successive beam spills, giving prampt information about problems with the apparatus as they arose. A version of the online analysis which read events from data tapes, allowed efficient; development and testing of the onl ine software.

3.20 Data Sample

- The experjment collected data for a total of 13 weeks between April and June of 1981 and 'January. and March of 1982, collecting 1500 data tapes. " 

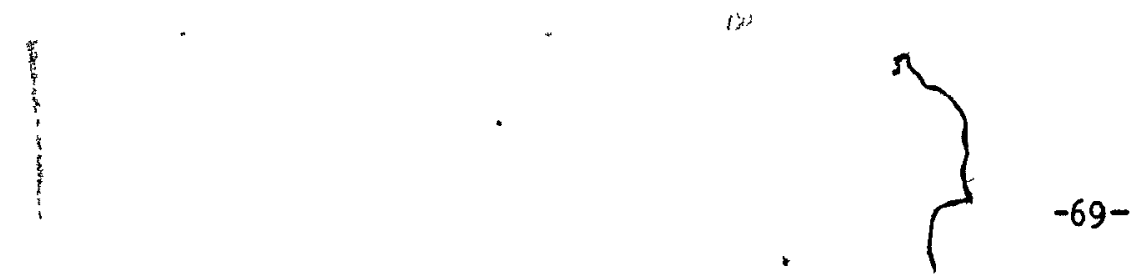

Most of the data was taken using a tungsten target in order to achieve the highest possible event rate. Ten percent of the running time was spent usthg a beryllium target, and 6 percent using a copper target, so that the A dependence of the "oross section for $\psi$ production by antiprotons and pions could be investigated. Of the 84 percent of the data "taken with tungsten targets, 28 percent was taken with the 1.0 interaction length target during the 1981 run. For the 1982 run, the length of the target was increased to 2.5 interaction lengths and 46 percent of the running time was spent using this configuration. In the final weeks of the 1982 run, the remaining 10 percent of the total running time was spent using a 0.4 interaction length tungsten target so that corrections for reinteraction of secondary.particles could be investigated.

During the course of the data taking, the performance of the șpectrometer was monitored online as described in a previous section. A subsample of the data tapes was also analysed offline as the experiment proceeded to :ensure that the apparatus was working correctlyn After the completion of the data taking, events on the complete set of data tapes were analysed and reconstructed as described in the next chapter. 


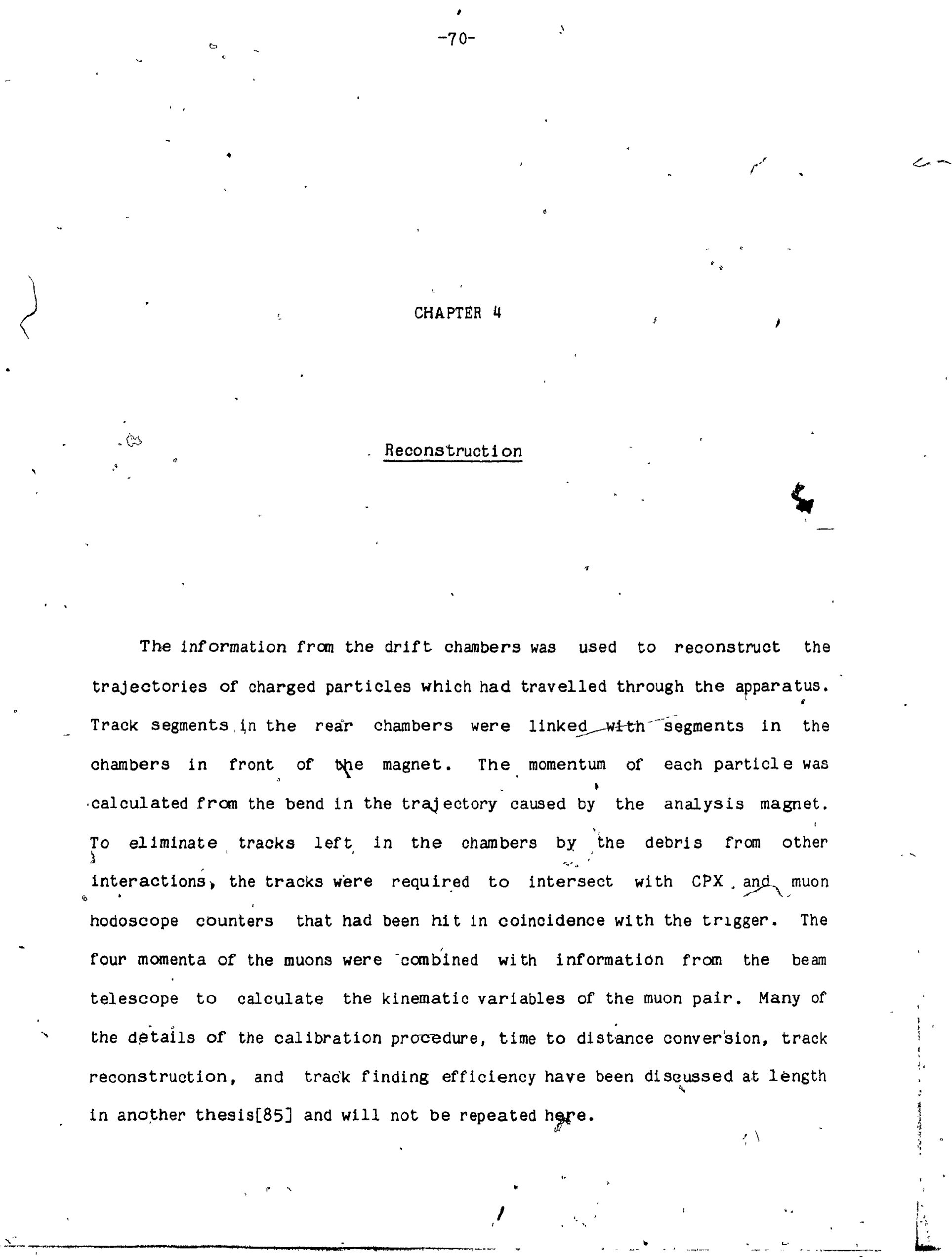


Recorstrugtion of the events from the data tapes took place in three stages. In the first stage of the analysis, each event was required to have two tracks in the chambers which pointed to muon triple coincidence channels that had been hit in coincidence with the trigger. The two tracks were required to give an invariant mass of at least $2 \mathrm{GeV} / \mathrm{c}^{2}$. If an event satisfied these critera the original event record was written to a secondary file known as a condensed file. A data tape with $1.4 \times 10^{5}$ triggers could be reduced to a file of 200 condensed events in $1500 \mathrm{CPU}$ seconds on the Fermilab Cyber 175 computer[86]. The condensed files from each run were collected on tape and subject to a second stage of analysis.

In the second stage, the events on the condensed data tapes were subject to the full analysis using both the drift chamber and beam telescope information. The track parameters from this stage of the analysis were written to a data summary tape without the original event redord. Each

- event required four times as much. CPU time at this stage as had been required for the previous condensation. The summary tapes al lowed events to be studied in detall without repeating the reconstruction each time.

In the final stage, the reconstructed tracks were required to pass through fiducial, regions corresponding to the physical apertures of the apparatus. Additional requirements were made on events with a high energy negative muon to reduce the contamination from beam halo. Events meeting all of these conditions were Iisted on a disk flle with their reconstructed kinematic variables, centre of mass energy, and trigger level requirement.

The entire chain of analysis programmes was checked using Monte Carlo events written in the original data event format with background hits incorporated from actual experimental data events. 


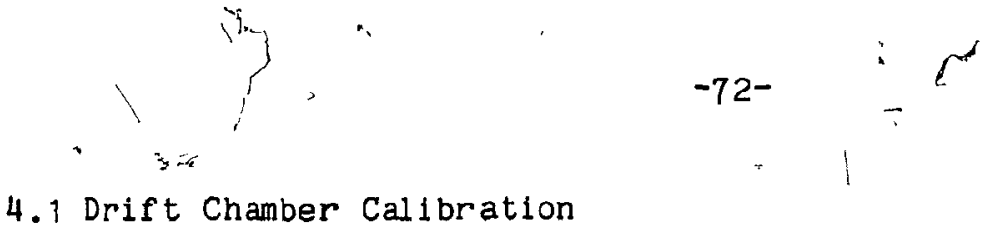

4. 3 Drift Chamber Calibration

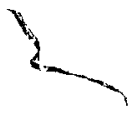

C,

The first step in the drift chamber track reconstruction involved the calibration of the time to digital converters (TDC'S) and the conversion of the drift time to drift distance. To a goòd approximation, the -drift velocity of electrons in the 50 percent argon and 50 percent ethane mixture used in this experiment is $50 \mu / \mathrm{ns}$ at atmospheric pressure independent of the electric field[72]. Because the drift paths are not always linearYland the drift velocity is not exactly constant across the cell, a better estimate of the time to distance conversion is obtained by integrating the time spectrum[87] as shown in Figure 20. If the chamber is illuminated by a uniform fiux of $N$ particles across a drift cell of width $\Delta x$, the time specțrum will be glven by.

$\frac{d N}{d t}=\frac{d N}{d x} \frac{d x}{d t}$

where

$\frac{d N}{d t}$ is the number of particles having a drift time $t$,

$\frac{d N}{d x}=\frac{N}{\Delta x}$ is the constant flux, and

$\frac{d x}{d t}$ is the drift velocity which may be a function of $x$.

By integrating both sides, the time fo distance conversion relationship can be expressed as

$x(t)=\frac{1}{N} \Delta x \int \frac{d N}{d t} d t$.

An initial analysis of a subset of the data tapes was made assuming a constant drift velocity across the cell. The time spectra of drift chamber hits used for the tracks reconstructed in this analysis were integrated to give a time to distance conversion relationship for each drift chamber plane. The relationships were stored as tablés on a disk file.

As discussed in the last chapter, each data tape began with a series of 


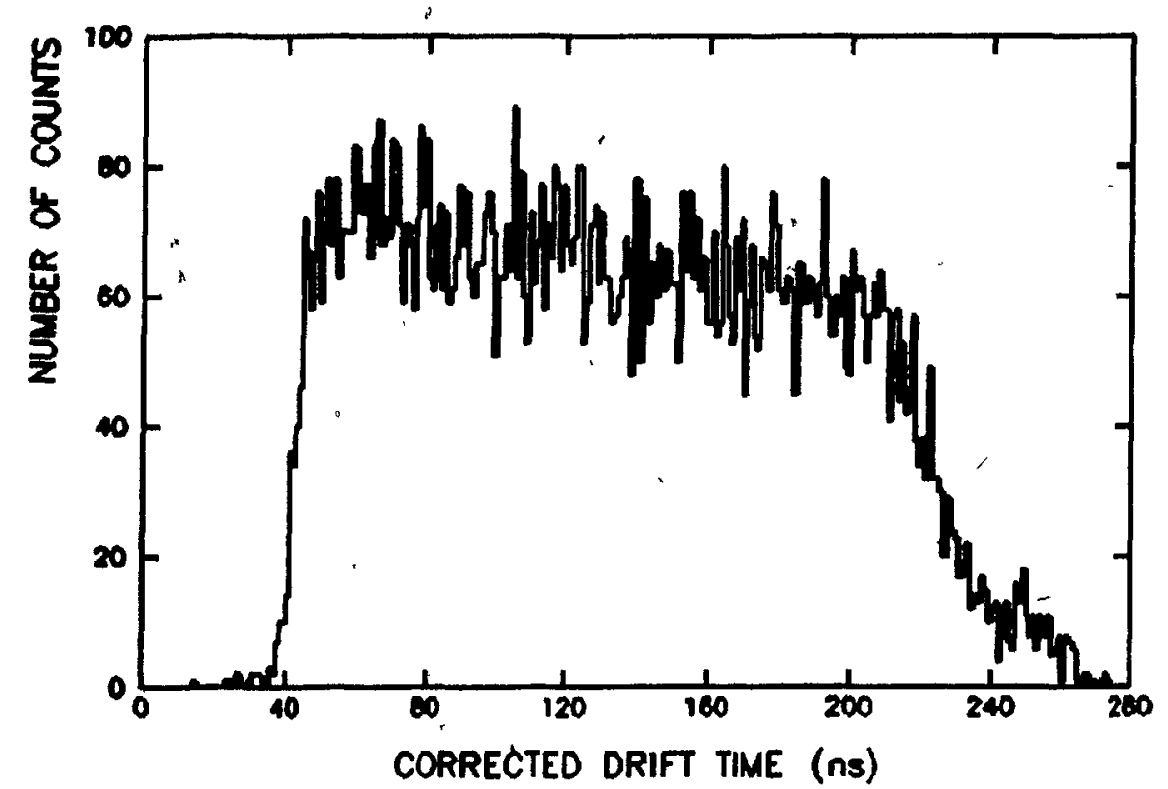

啱

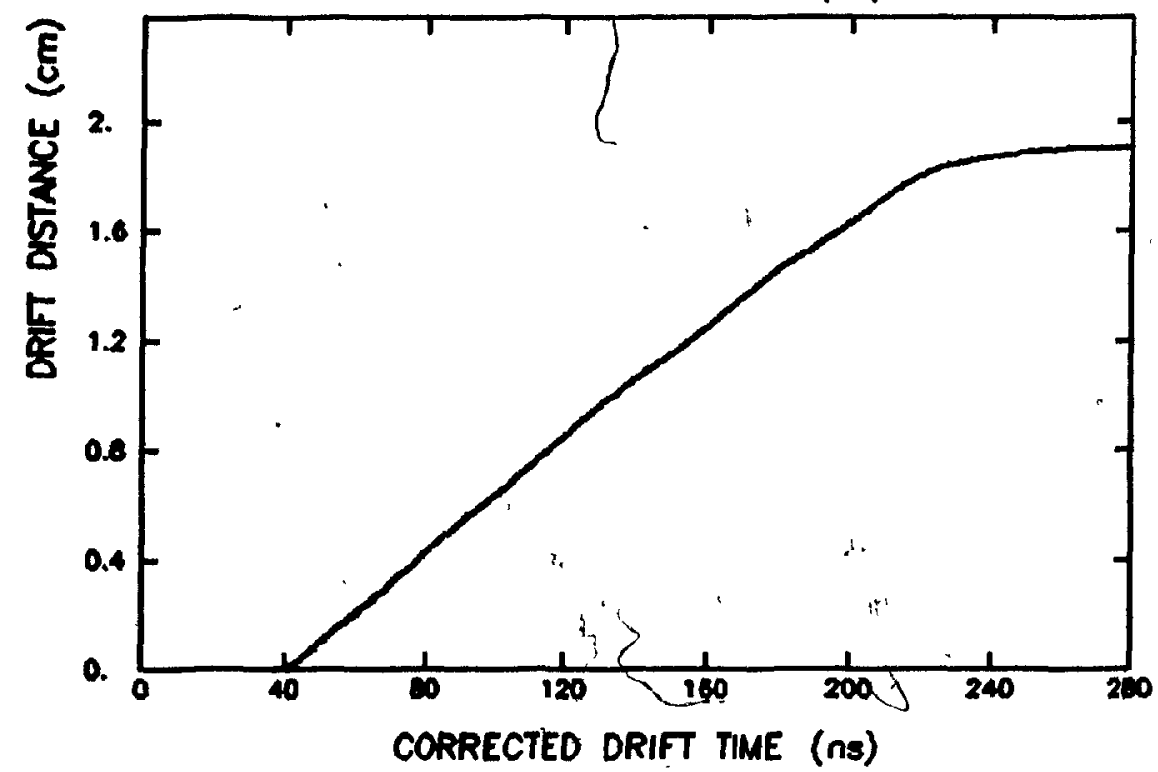

4

$$
\text { Figure 20 - Time to Distance Conversion }
$$

The top histogram shows the number of tracks plotted against the corrected drift time for the $X$ plane of drift chamber DC4. The lower figure showis the integrated time distribution normalized to give the time, to distance conversion as discussed in the text. 
six hundred calibration events. Frior to the analysis, the calibration events were read and straight $l_{c}$ lnes were flt to the number of counts vs delay curve for each TDC. As the TDC information was read for each event, the calibration constants for each channel were used to convert the counts to drift time. The drift time was used to look up' the distance the track had passed from the sense wire.

4.2 Drift Chamber Reconstruction"

The drift chamber track reconstruction proceeded from the rear of the spectrometer to the front: The chambers downstream of the magnet typically had half, as many wires hit per plane for a given event, and this made track finding simpler. Track segments in the rear were projected through the magnetiand used to help find the coresponding hits in the front chambers. The track momentum was calculated from the bend of the track in the analysis magnet and combined with the direction of the upstream track segment to give the four momentum of the muon in the laboratory frame. The charge of each particle was determined from the direction of the magnetic deflection.

The information from each of the drift ahambers in the rear was studied in turn and all pos'sible combinations of hits were examined to find intersections of $U$ 'and $V$ "wire hits that colnofided with an $X$ wire, hit as shown. schematically in Figure 21. The combination was deemed to be a rough triplet if

$$
\left|X-\cos \theta \frac{U+V}{2}\right| \leq 0.8 \Delta X
$$

where

$X, U$ and $V$ are the coordinates of the wires as measured from the $Z$ axis as shown in the figure, 


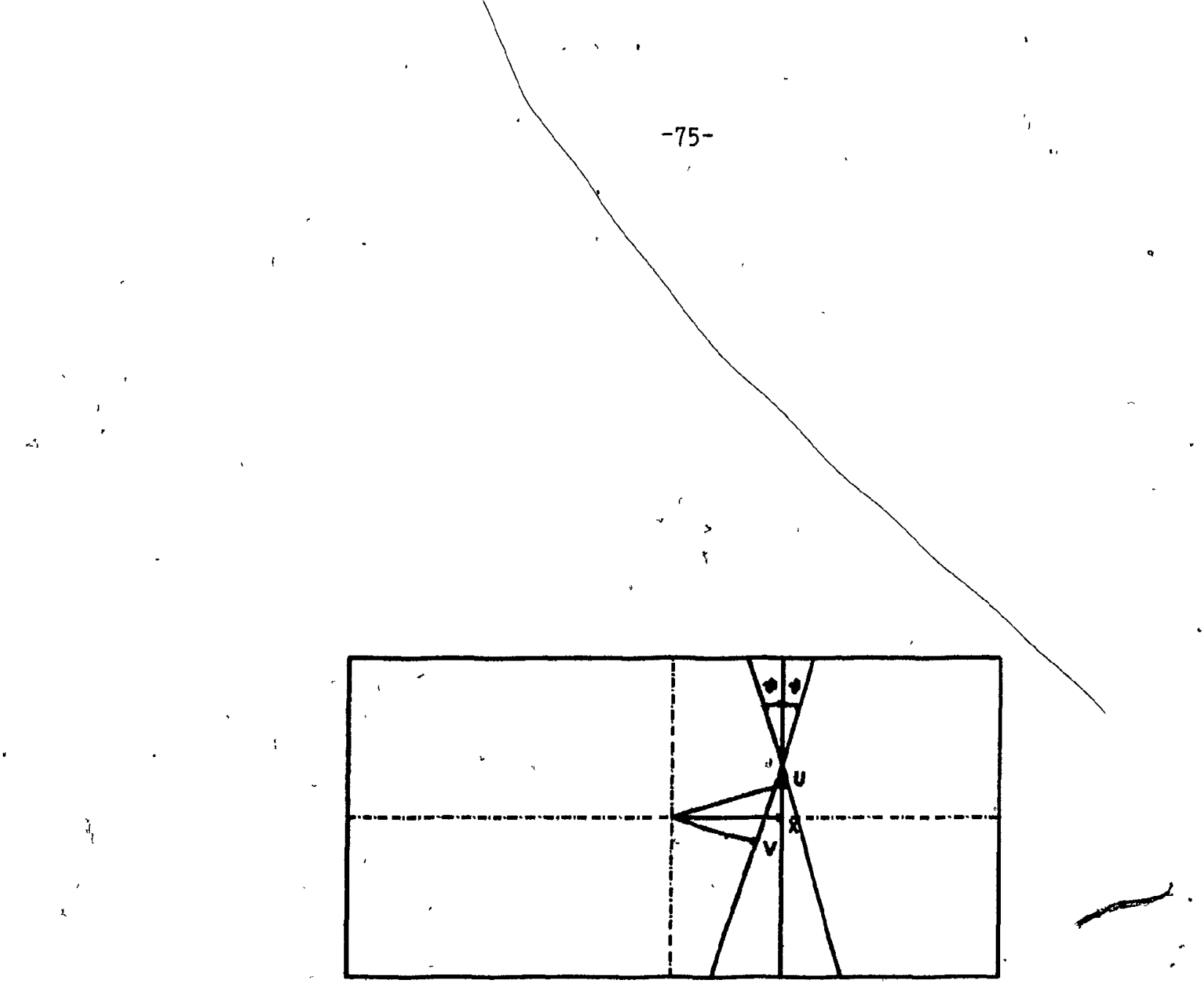

Figure 21 - Drift Chamber.Triplets

The intersections of hit wires in the $X, U$, and $V$ planes of a chamber were used to define a drift chamber triplet as discussed in the text. The $X, U$ and

$i \quad V$ coordinates were measured from the centre of the chamber as indicated by the arrows. 
$\Delta \mathrm{X}$ is the chamber wire spacing, and

$\theta=\tan ^{-1} 0.3$ is the stereo angle from the vertical of the $U$ and $V$ planes in the drift chambers.

At this stage only the wire coordinates were used; no information about the drift time was included. Up to 40 rough triplets were allowed in each chamber.

The next step was to combine the rough triplets in the three chambers downstream of the magnet to $f$ ind all pössible track segments. Three triplets, one each in chambers DC4, DC5 and DC6, were deemed a rough track segment (no drift time information used) if

$$
\left|x_{5}-\frac{x_{4}+x_{6}}{2}\right| \leq 1.1 \Delta x
$$

and

$$
\left|Y_{5}-\frac{Y_{4}+Y_{6}}{2}\right| \leq 1: 1 \Delta X / \tan \theta
$$

where

$X_{i}$ and $Y_{i}$ are the coordinates of the triplet in chamber DCi, and. $\Delta X$ and $\theta$ are the wire spacing and stereo angle as discussed above.

After all track segments with three rough triplets were found, the programme returned to find any track segments that could be constructed using two rough triplets and a pair of wires in the remaining chamber. Thus a track segment required that at least 8 of the 9 possible hits be found before it could be reconstructed. Again up to 40 rough track segments were allowed.

At this point, the time information was included to refine the rough triplets. The drift time was calculated from the digitizer counts using the calibration constants discussed in the last section, and was used in turn to calculate the distance the track had passed from the sense wire. The slopes of the rough tracks were used to project the hits in the $U$ and $V$ planes onto 
the corresponding $X$ plane. When the time information was included, each set of three hit wires could be used to constcuct $2^{3}$ possibie fine triplets because of the.left-right ambiguity inherent in the drift time. The $X$ and $Y$ positions of each possible fine triplet were calculated as

$$
X_{\text {Triplet }}=(V+X+U) / 3
$$

and

$$
Y_{\text {Triplet }}=(V-U) /(2 \tan \theta)
$$

where $X, U$ and $V$ are the coordinates of the drift chamber hits projected onto the appropriate $X$ plane with the time information included. The sum of the 'squared residual distances, $R^{2}$, of the $X, U$ and $V$ hits from the position of the fine triplet, that is,

$$
\therefore \quad R^{2}=\left(X-X_{\text {Triplet }}\right)^{2}+\left(U-U_{\text {Triplet }}\right)^{2}+\left(V-V_{\text {Triplet }}\right)^{2}
$$

was required to be less than $0.016 \mathrm{~cm}^{2}$ where the $U$ and $V$ positions of the triplet were given by the following expressions:

$$
\begin{aligned}
& U_{\text {Triplet }}=\left(X_{\text {Triplet }}+Y_{\text {Triplet }} \tan \theta\right) ; \text { and } \\
& V_{\text {Triplet }}=\left(X_{\text {Triplet }}{ }^{-Y} \text { Triplet } \tan \theta\right) .
\end{aligned}
$$

Again $\theta$ is the stereo angle of the $U$ and $V$ planes. The residuals of the fine triplets in the rear chambers typically had an RMS value of $0.25 \mathrm{~mm}$. The fine triplets were in turn used to define fine tracks in the rear.

- The remaining track segments in the rear were then projected through the magnet to the plane of the third drift chamber. The $Y$ coordinates of rough triplets in the chamber were compared to the $Y$ coordinate of each projected track in turn and. fine triplets were constructed if a match was found. Rough track segments in the front were constructed by linking the fine triplets in the thlrd chamber with the intercept at the magnet bend plane of the track segment in the rear. The candidate track segments in the front were projected to the first and second chambers which were then 


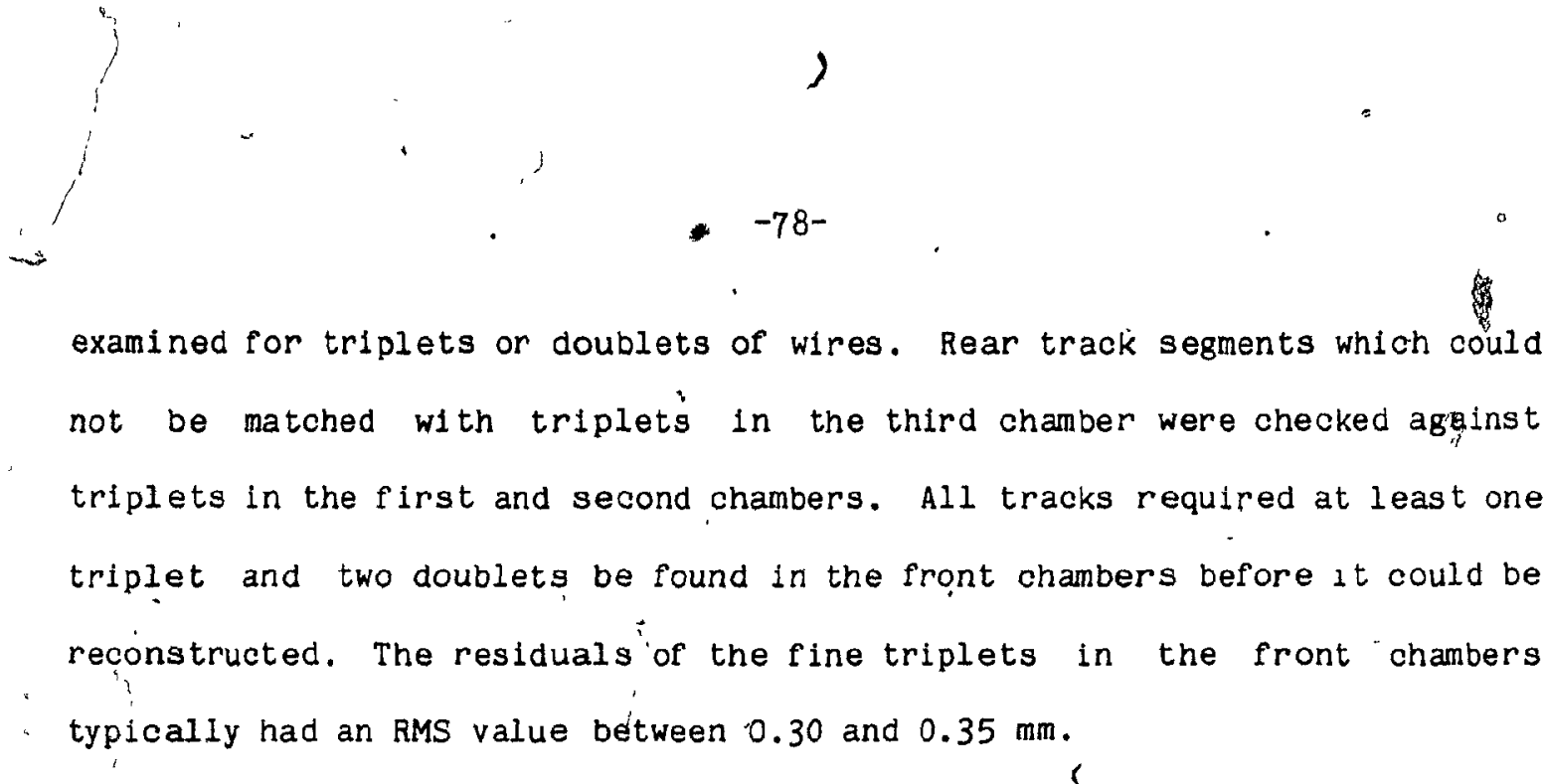

The horizontal momentum component of each track was then calculated by fitting a circular are of radius

$$
\rho=L_{E f f^{\prime}}\left(\sin \theta_{I N}-\sin \theta_{O U T}\right) \text {, }
$$

as shown in Figure 22, between the $X$ projections of the front and rear track" segments and ysing [13]

$$
\mathrm{p}_{X-Z}=0.3 \mathrm{H} \rho / \mathrm{c}
$$

The quantities appearing in these equations are defined"äs follows:

${ }^{\theta}$ IN is the angle between the the $X-Z$ projection of the track upstream. of the magnet and the $\mathrm{Z}$ axis;

$\theta_{\text {out }}$ is the angle between the the $x-z$ projection of the track downstream of the magnet and the $Z$ axis;

$L_{\text {Eff }}$ is.the effective length of the field;

$\mathrm{p}_{X-Z}$ is the component of momentum in the $X-Z$ plane measured in MeV/c; . H is the magnetic field in Kgauss; and

$\rho$ is the raditis of curvature of the track in centimetres.

Corrections were applied for the energy loss of the muons in the copper absorber[88], and the four momentum components of each muon were calculated from $p_{X-Z}$ and the direction of the upstream track segment as $p_{X}=p_{X-Z} \sin \theta_{I N}$

$p_{Y}=p_{X-Z} \cos \theta_{I N} \frac{d Y}{d Z}$

$p_{Z}=P_{X-Z} \cos \theta_{I N}$, and 


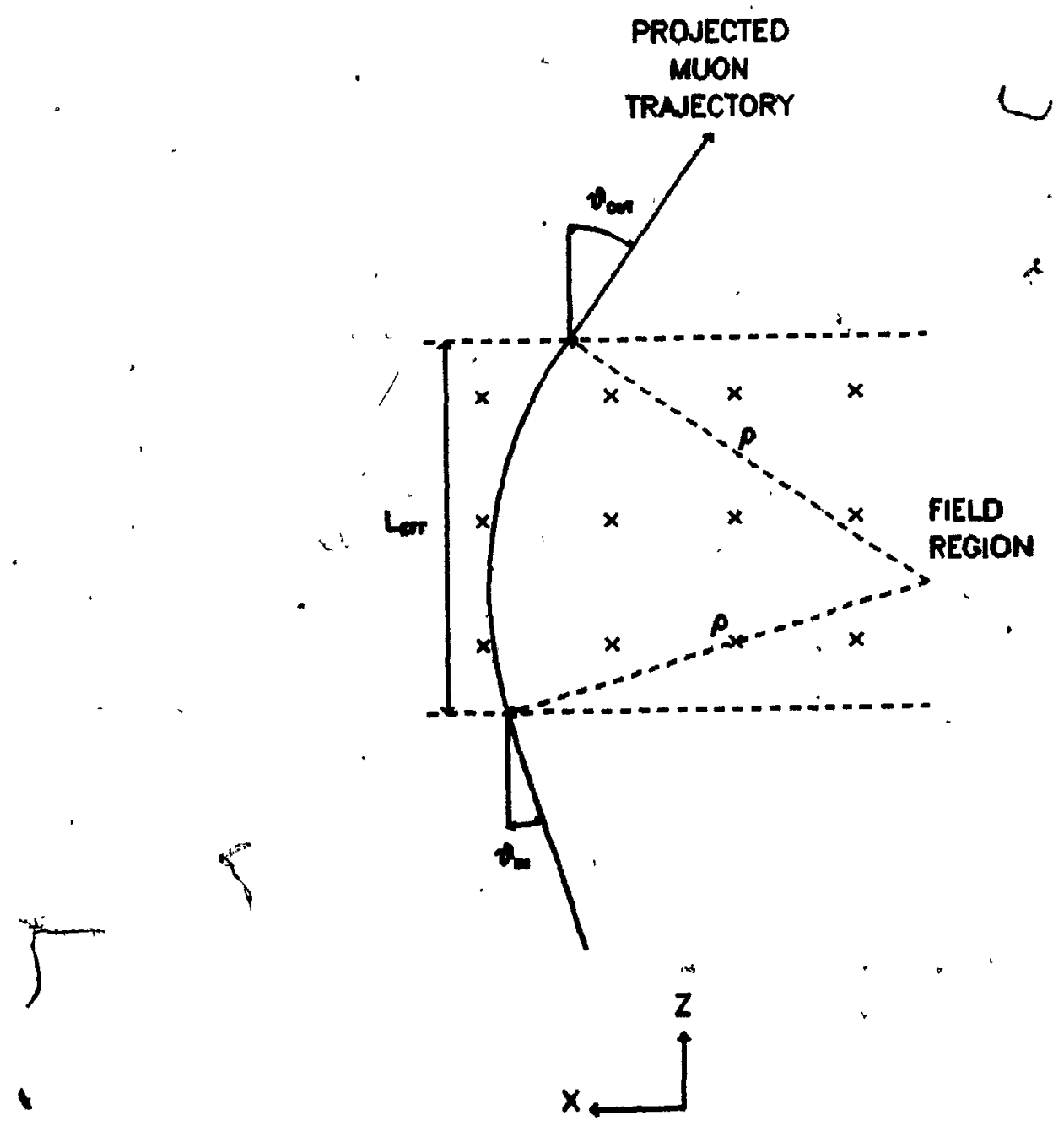

\section{Figure 22 - Momentum-Calculation} The momenta of the tracks were calculated using a square field approximation
as discussed in the text. 


$$
E=\left(p_{X}{ }^{2}+p_{Y}^{2}+p_{Z}{ }^{2}+M_{\mu}{ }^{2}\right)^{1 / 2}
$$

where $\frac{d Y}{d Z}$ is the $Y$ slope of the upstream track segment. The charge of each muon was determined from the direction of the deflection in the magnetic fièld.

\subsection{Beam Chamber Reconstruction}

The large momentum spread and spatial divergence of the beam made it necessary to measure the position and momentum of each individual beam particle. Information from the beam chambers and the beam hodoscope counter elements was used to reconstruct the beam, particle trajectories. The, particle momenta were calculated from the bend in the trajectories caijsed by the magnets in the beam spectrometer.

Wires hit in the nine planes" of beam proportional champers' were "examined to find triplets in each. of the three beam stations as shown schematically in Figure 23. Correlated triplets in the three stations "were matched in the non-bend or $x$ coordinate to $f$ ind tracks. The momenta of the tracks were calculated using a single bend plane approximation for the pair of dipole magnets in the beam spectrometer. Candidate tracks outside of the acceptance of the beam telescope or of the momentum bite of the beam were. rejected.

As discussed in the last chapter the beam counter hodoscopes were designed to have a sensitive time shorter than the separation between successsive RF buckets. Residual tracks in the chambers due to particles not in coincidence with the trigger were eliminated by requiring the trajectories to point at beam hodoscope elements which had recorded a count.

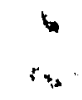




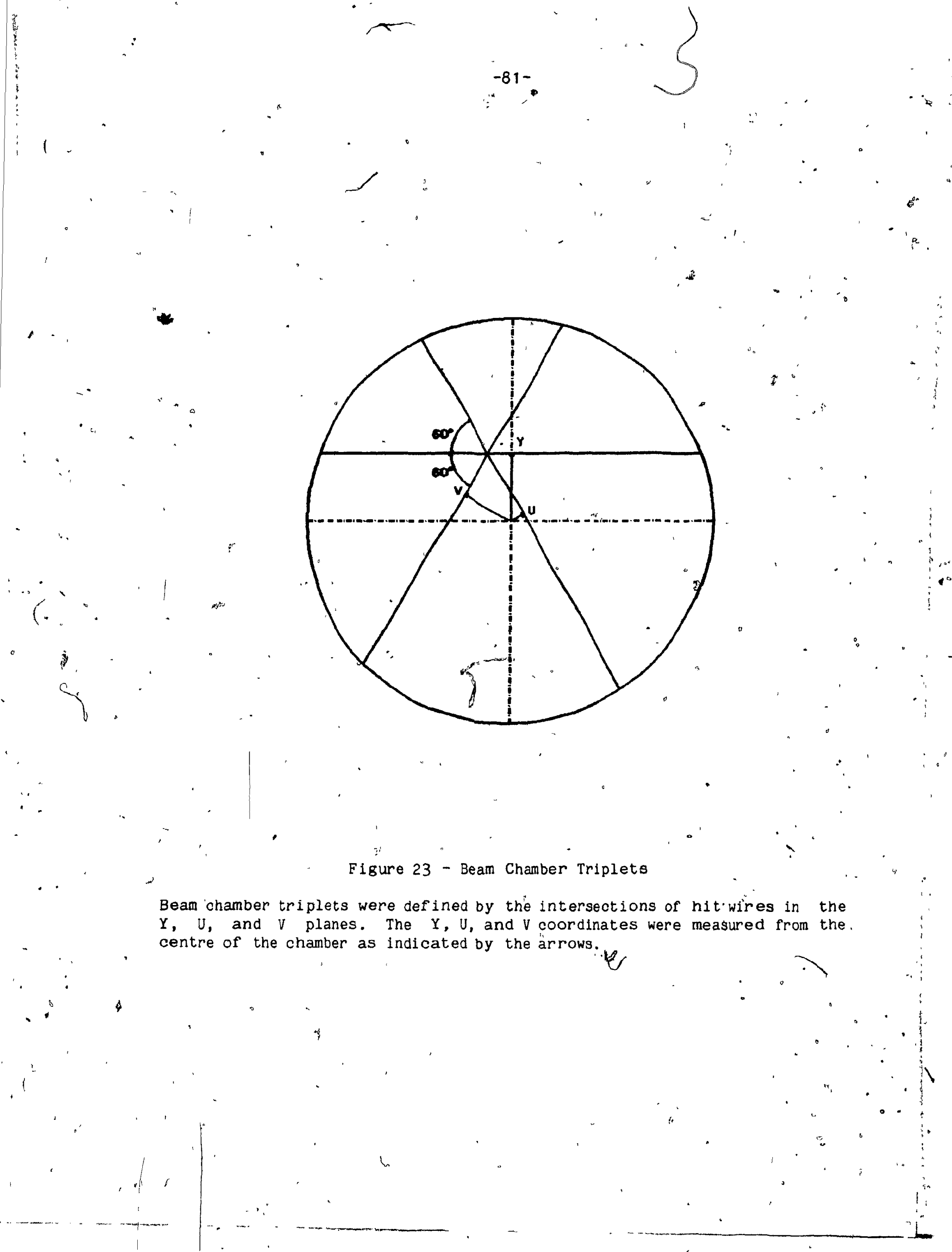


. The beam momentum spectrum and profile are shown in-Figure 24: The .. momentum spread of the beam was $15 \mathrm{GeV} / \mathrm{c}$ "FHM spatial spread of $10 \mathrm{~cm} \mathrm{FWHM} \mathrm{in} X$ and $6 \mathrm{~cm}$ FWHM in $Y$. The beam is not centred about $Y=0$ at the target because of the upward bend caused by the momentum tagging magnets. The tagging system allowed the momentum to be measured to $1 \mathrm{GeV} / \mathrm{c}$ and the posit $\mathrm{o}^{\circ}$ to $0.5 \mathrm{~cm}$ FWHM at the target position. 4.4 Vertex and Halo Requirements

Pairs of reconstructed tracks were projected upstrean through the beam dump to determine whether the collision had occured in the target or the dump. The point at which the distance between the tracks was a minimum was used as a first estimate of the production vertex. Information from the drift chambers, beam chambers, and the absorber chamber was used to make a better estimate of the vertex using the algorithm discussed in Appendix II. A histogram of the $z$ position of the reconstructed vertices for pion induced events in the 1982 sample is shown in Figure 25, and the position of the target and the upstream end of the dump are indicated. The vertex reconstructed in this way allowed events produced in the dump to be clearly separated from events orlginating in the target.

Requirements were placed on the distance between the tracks at the reconstructed vertex and on the position of the vertex to ensure that the two muons did originate at a common point fand were not the result of an, accidentâl. coincidence between a halo particle and a muon from the decay of a hadron. The reconstructed vertex was required to be within $9.144 \mathrm{~cm}$ in $x$ and $10.16 \mathrm{~cm}$ in $\mathrm{Y}$ of the nominal beam spot at the target, that is, $X=0.0$ and $Y=5.08 \mathrm{~cm}$. The $Z$ position of the reconstructed vertex was required to 


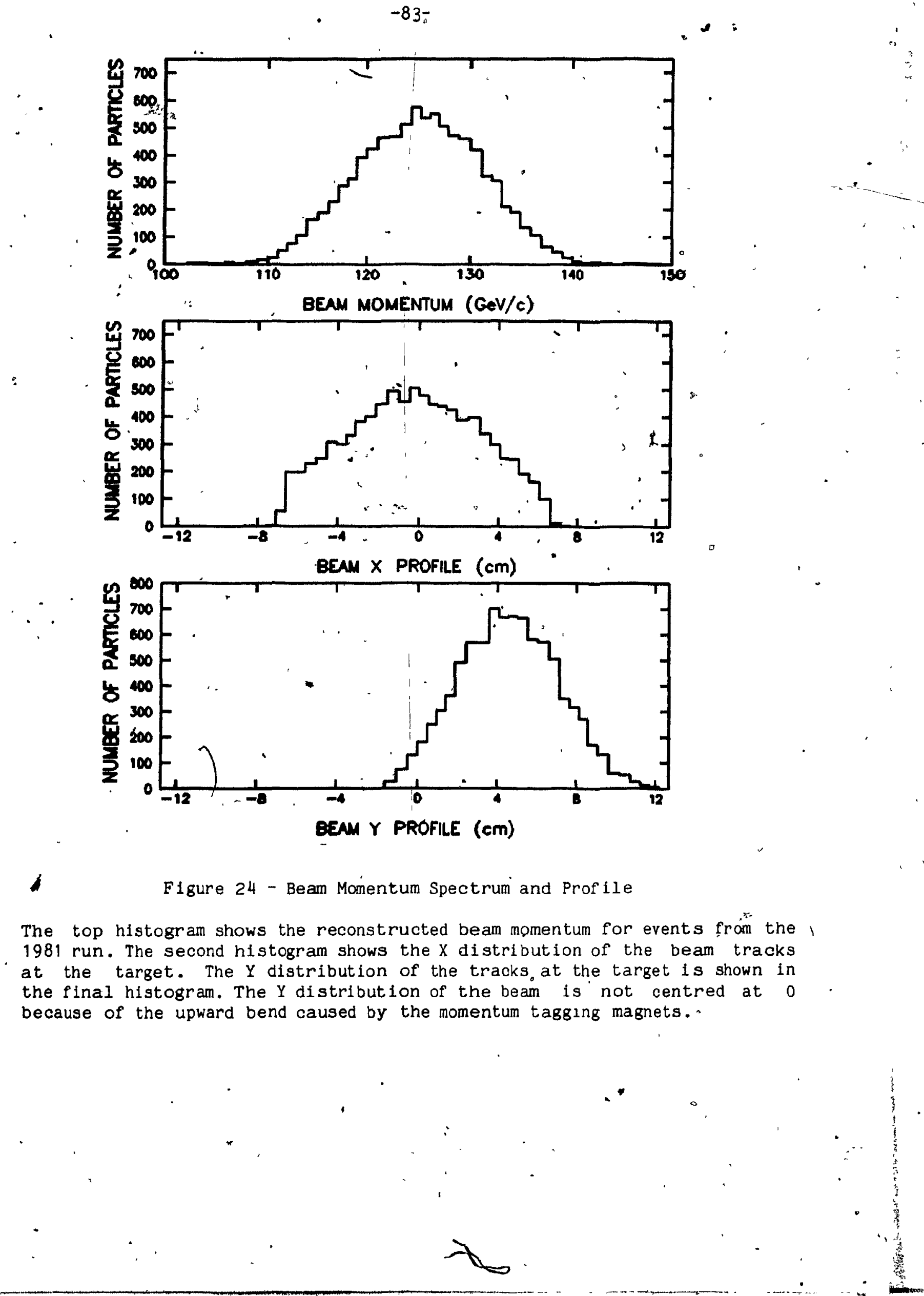




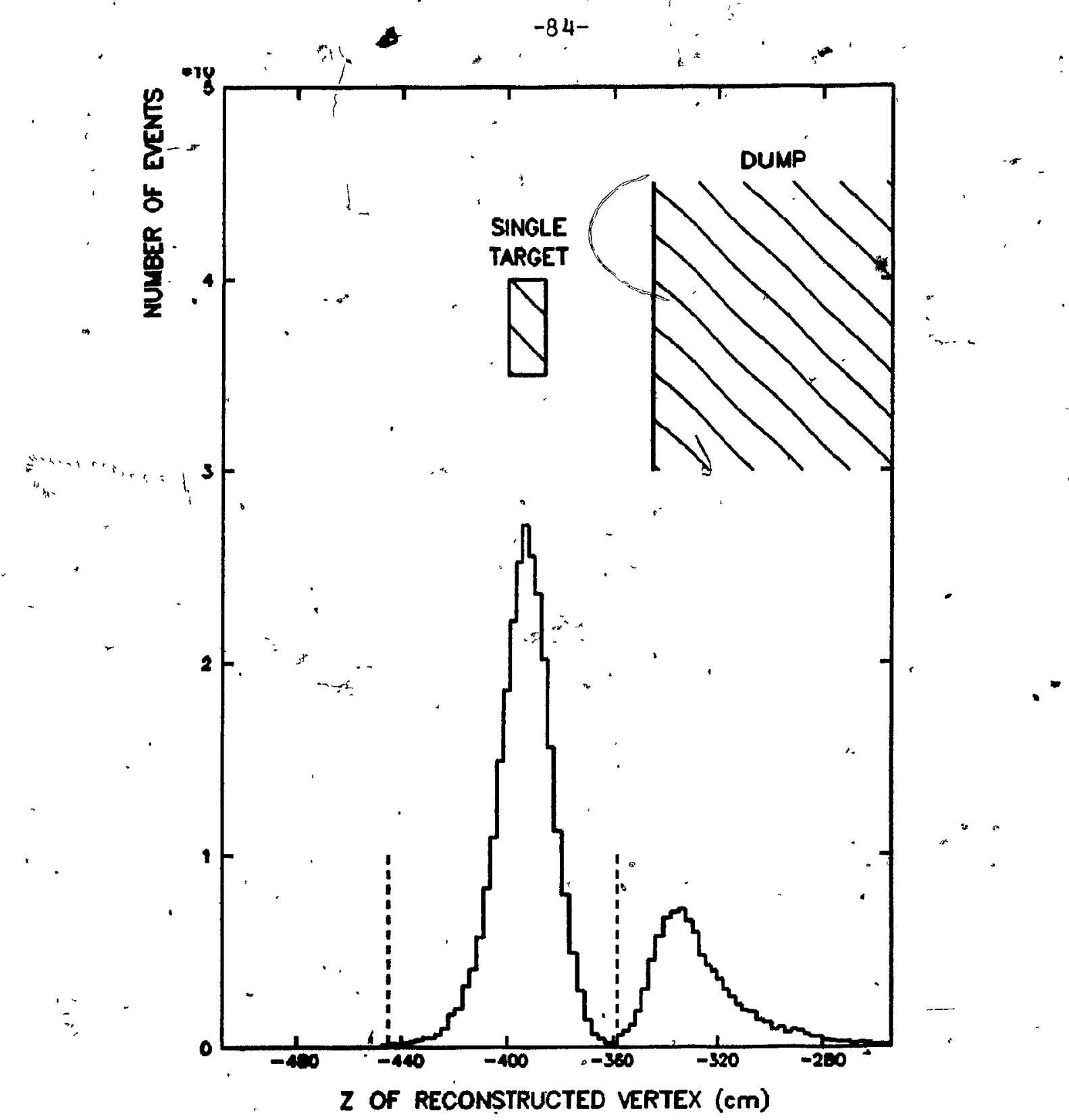

Figure 25 - Reconstructed Vertex.

The reconstructed $Z$ vertex position is shown for the tungsten target used during the 1982 run. The positions of the target and the copper beam dump are indicated in the top half of the figure, and the dashed lines show the positions of the vertex cuts. The reconstructed vertex was used to assign the events to either the dump or the target. The kinematic variables for events assigned to the target werer recalculated assuming that they had originated at the centre of the target. 
be between $-444.3 \mathrm{~cm}$ and $-358.14 \mathrm{~cm}$, when the single target was used, and" between $-495.5 \mathrm{~cm}$ and $-358.14 \mathrm{~cm}$ when a split target was used. The distance of closest approach of the two tracks was required to be less than $7.62 \mathrm{~cm}$ in $X,-10.16 \mathrm{~cm}$ in $Y$, and $2.54 \mathrm{~cm}$ in $Z$. When these vertex requirements were ${ }^{\circ}$ tested in the Monte Carlo simulation, they resulted in a loss of less than 1 percent of the events with no bias in any of the kinematic variables.

- To eliminate any residual contamination by beam halo, special requirements were imposed on events with a high momentum negatively charged muon to ensure that both muons came from a common vertex. Events with a muon of either charge with a momentum of greater than $85, \mathrm{GeV} / \mathrm{c}$ were not included in the final data sample. Events with a negative"muon with a -momentum of greater than $20 \mathrm{GeV} / \mathrm{c}$ were excluded from the final data sample $1 f^{\circ}$ the separation of the tracks at the target was greater than $5.08 \mathrm{~cm}$ and If the distance of elther trask from the reconstructed beam track was greater than $5.08 \mathrm{~cm}$.

The special, vertex requirements Imposed on events with a high momentum negative muon were studied lising the Monte Carlo simulation and by applying * them to events with a high momentum positive muon and found to result in a negligible loss. The rejection of events with a muon having a momentum of greater than $85 \mathrm{GeV} / \mathrm{c}$ resulted in the loss of a few percent of the oppositely charged high mass events, but eliminated'a large fraction of the negative-negative events surviving the other requirements. This requirement had no effect on the kinematic distributions with the exception of the high $x_{F}$ region. Figures showing the effects of this requirement are presented together with the track $f$ inding efficiency in a later section. A correction for these effects was included in the calculation of the acceptance, as iffscussed in the next chapter. 


\subsection{Aperture and Trigger Requirements}

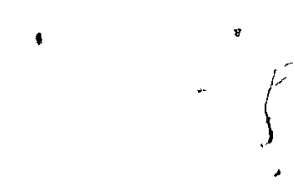

The reconstructed muon tracks were required to pass through fiducial regions corresponding to the defining apertures of the apparatus. In addition, the track's were required to point at counters that had been hit in colncidence with'the trigger. The muon pair was also required to completely satisfy the trigger conditions that had been imposed during the data taking .

These requirementş eliminated most of the events which had satisfied the trigger because of an accidental coincidence between a Hadron decay or punch through and a beam halo particle, and guaranteed that counter inefficiencies could be accurately calculated. Because the resolving time of the counters was much smaller than the sensitive time "of the drift chambers, it was possible to eliminate tracks from interactions of beam particles in previous RF buckets. The aperture and trigger requirements also ensured that the acceptance of the apparatus could be accurately modelled by making the same requirements in the Monte Carlo.

The $X$ and $Y$ coordinates of the reconstructed tracks were calculated at 2 for varlous values of $z$ as listed in Table 5;. These correspond to the following physical locations:

1. the edges of the active area of the third drift chamber, $-2$

2. the aperture defined by the downstream end of the magnet yoke,

3. the downstream edges of the magnet colls, 



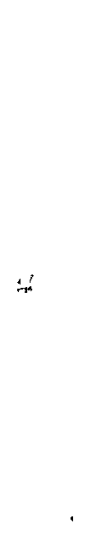

4. the edges of the active areas of "the fourth' and sixth chambers,

5. the edges the beam hole in the CP, and CPY counters, and

6. the edges of the beam hole in the first muon hodoscope.

At the drift chambers and magnet apertures the track coordinates were examined and the event was rejected if 'they " passed outside "of a rectangular region of size $\pm D X$ and \pm DY centred on the nominal beam centre at $X_{O}$ and YO, also listed in Table 5. At the CPX, CPY, and muon hodoscope holes the tracks were rejected if they passed intside of a rectangular region of size $\pm D X$ and $\pm D Y$ centred at $X O$ and YO. Because the active areas of the chambers were not square, the tracks werealso required to fall within a region $\pm D U$ and $\pm D V$ of the nominal beam centre spot at the third and sixth drift chambers, where $U$ and $V$ are defined as

$$
\begin{aligned}
U & =X+Y \tan \theta, \\
V & =X-Y \tan \theta \text {, and } \\
\theta & =\tan ^{-1}(0.3) \text { is the stereo angle of the } U \text { and planes of the rdrift } \\
& =\text { chambers. }
\end{aligned}
$$

Both reconstructed muon tracks were required to point to CPX, CPY and muon triple coincidence channels which had been hit in coincidence with the trigger. Half a counter spacing was allowed for resolution at the CPX and: the CPY counters and a $12.7 \mathrm{~cm}$ margin for multiple scattering was allowed with the muon counters. The two tracks were required to point to muon triple coincidence channels in different quadrants of the hodoscopes. Each muon was required to point to a different $C P X$ counter with a recorded hit to guarantee that the CPX 2 requirement of the hardware trigger was satisfied. 
in addition at least one of the muons was required to pass outside of a circular region corresponding to the absorber counter hole if the trigger for a given event had required the counter. The absorber counter quadrants traversed by the reconstruçted tracks were compared to the list of active F counters and at least one of the quadrants hit was required to have recorded a count

4.6 Reconstruction Efficiency

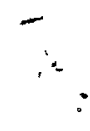

$\therefore$ It is difficult to make reconstruction-programmes one hundred percent effičent. Missing hits due to chamber inefficiencies, electronlcs dead time and extra tracks from halo particles or other interactions can confuse even the most carefully written programmes. Our reconstruction ef ficiency was investigated by generating Monte Carlo events with simulated backgrounds and inefficiencies and reconstructing them with the analysis programmes.

Background hits in the chambers were simulated by using the drift chamber information from events on a'special data tape taken using the BEAM "signal only as the trigger. Muon pairs generated by the Monte Carlo. simulation were propagated through the apparatus using its measured parameters and added to the background drift chamber hits. The drift chambers were assumed to be 99.5 percent efficient, a number consistent with tests using cosmic rays ánd low initensity beam runs. The dead time of the drift chamber electronics was included by disabling drift chamber wires which had beeff hit in a second beam event. These simulated data events were anal ysed wit the same programmes as the data events, and the reconstruction ef ficiency fas calculated by comparing the number of events generated to the number sy viving the analysis. 


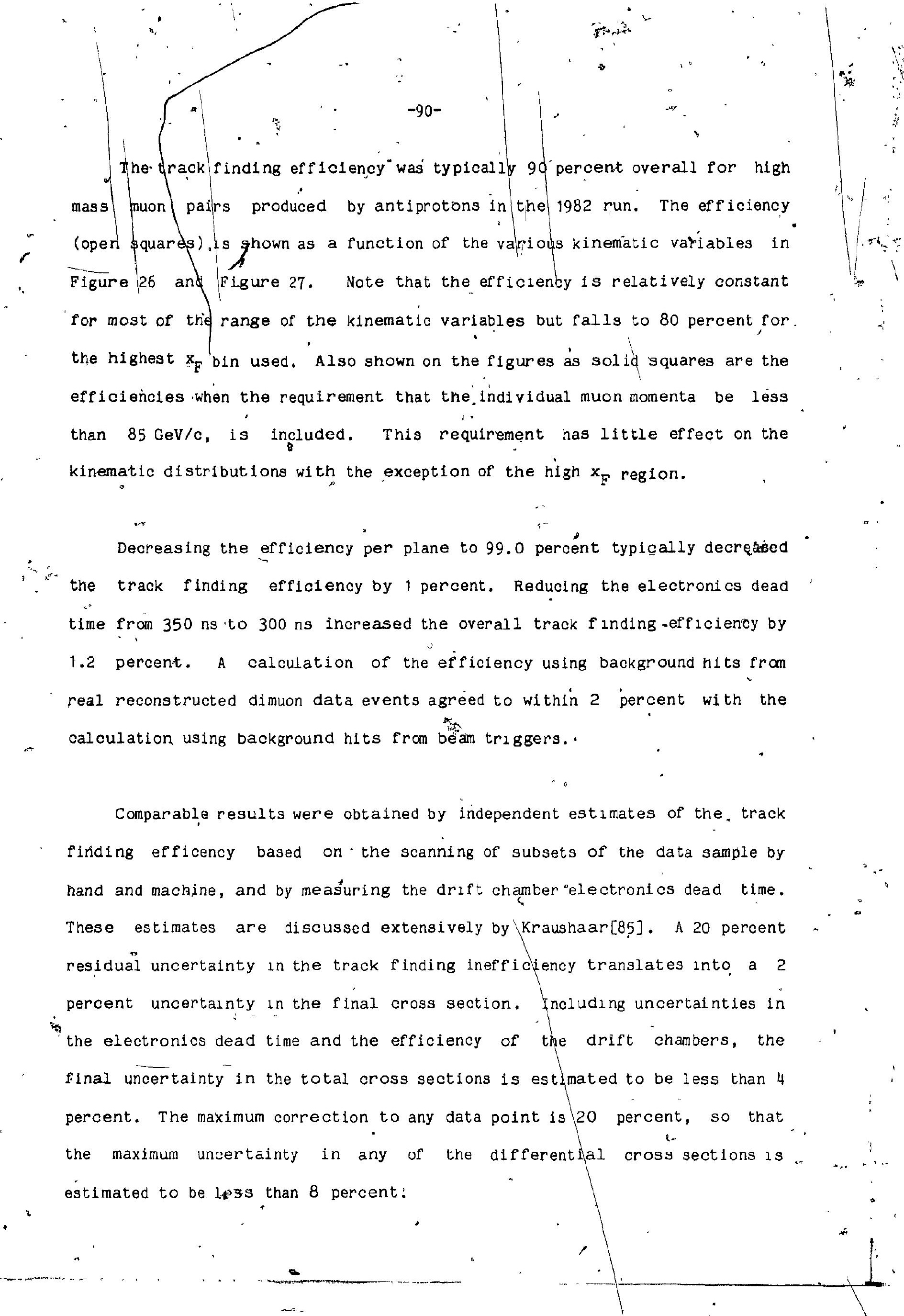



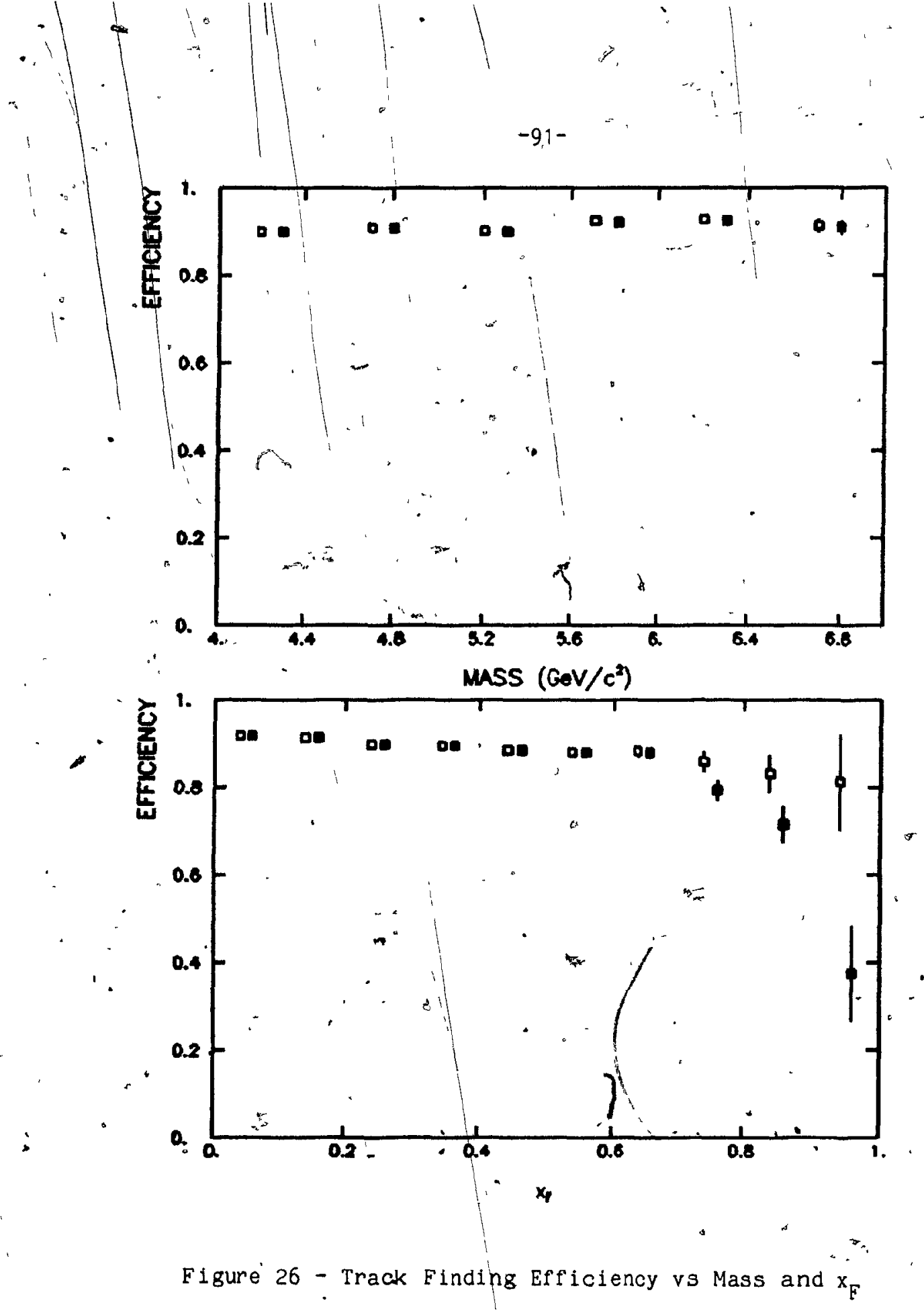

The open squares show the track finding efficiency as functions of mass and, $\mathbf{x}_{F}$ for the 1982 running period. The solid squares show the combined ef fects of track finding efficiency and the kinematic requirements imposed on the reconstruated events. The requirement that both muons have a momentum of less than $85^{\circ} \mathrm{GeV} / \mathrm{c}$ results in the loss of half the events in the highest $x_{F}$
bins. 

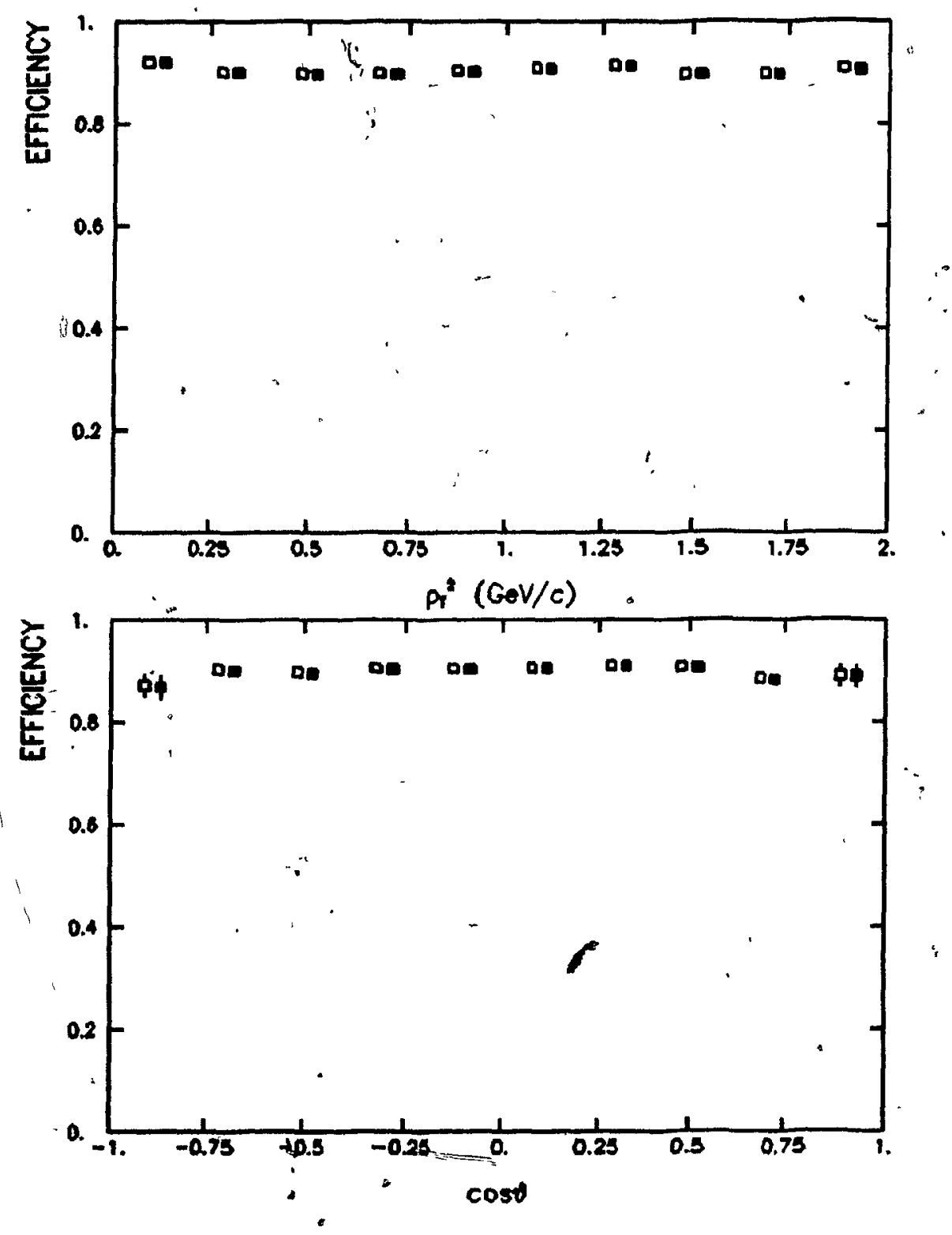

E.igure 27 - Track Finding Efficiency $v s, \mathrm{p}_{\mathrm{T}}{ }^{2}$ and $\cos \theta$

- The open squares show the track finding efficiency as functions of $p_{T}{ }^{2}$ and $\cos \theta$ for the 1982 running period. The solid squares show the combined ef fects of track finding efflciency and the kinematic requirements imposed on the reconstructed events, There is no strong dependence of the reconstruction efficiency on any of these kinematic variables. 
The acceptance of the spectrometer was calculated by generating events with the simulation programme and determining what fraction. of them successfully traversed the entire spectrometer. The details of the simulation programme will be discused in the next chapter. The reconstruction efficiency was incorporated by requiring the Monte Carlo events, including background and electronigs dead time as decribed here, to be reconstructed with the same series of analysis programmes as the real experimental data.

4.7 Kinematic Reconstruction and Data Sample

The mass resolution of the spectrometer was limited by the uncertainty in the opening angle of the muon pair caused by multiple scattering of the muons in the copper absorber. In the final stage of the analysis, inf ormation from the beam chambers and the absorber wire chamber was used to determine the opentng angle of the muon pair and thereby give the best resolution for the spectrometer. The four momenta of the muons were then recalculated using this procedure and used to calculate the kinematic variables of the muon pair.

The muon pairs could be assumed to have been created by the interaction of a beam particle in the target. Monte Carlo simulations showed that the measurement of the opening angle could be juproved by assuming that the muons had originated at the centre of the target rather than just using the drift chamber tracks to determine the $z$ position of the vertex. The beam track was used to provide the $X$ and $Y$ coordinate positions of the production vertex. The absorber chamber gave information about the positions of the tracks before they had been fully scattered, and could be used to give the 


\section{tri} tracks in the front of the magnet were projected back to the centre plane of. the absorber chamber. A radial area of $1.016 \mathrm{~cm}$ around the tracks. was searched for hits. The $X$ and $Y$ positions of the track in the absorper \chamber were taken to be the crossing point of the $U$ and $V$ wires closest/to the track. Line segments linking the absorber chamber coordinates with/the centre of the target segment in which the event was produced were used to give the final values of the production angles of the muons.

The effects of different reconstruction procedures on the width of the $\psi$ peak were examined. Using only the tracks'in the front drift chambers to calculate the production angles of the muons gave a $\psi$ peak with a wath of $270 \mathrm{MeV} / \mathrm{c}^{2}$. Using line segments linking the drift chamber tracks in the front chambers to the centre of the target segment gave a width of $190 \mathrm{MeV} / \mathrm{c}^{2}$, Incorporating the absorber chamber information reduced the width of the peak to $185 \mathrm{MeV} / \mathrm{c}^{2}$.

The four momenta of the muons were recalculated using the best values for the direction of each muon. The beam-target centre fo mass was. calculated from the measured four momentum of the beam particle assuming that the target particle was a nucleon at rest in the lab frame. The muons were Lorentz transformed to the centre of mass frame and used to calculate the Invariant mass, $x_{F}, P_{T}$, and rest frame parameters of the pair as described above in Chapter 2. The individual muons and the beam and target particles were transformed from the centre of mass frame to the rest frame of the muon pair and the angular variables, $\theta$ and $\phi$, were calculated using "s. the Gottfreid-Jackson convention for the $z$ axis. 


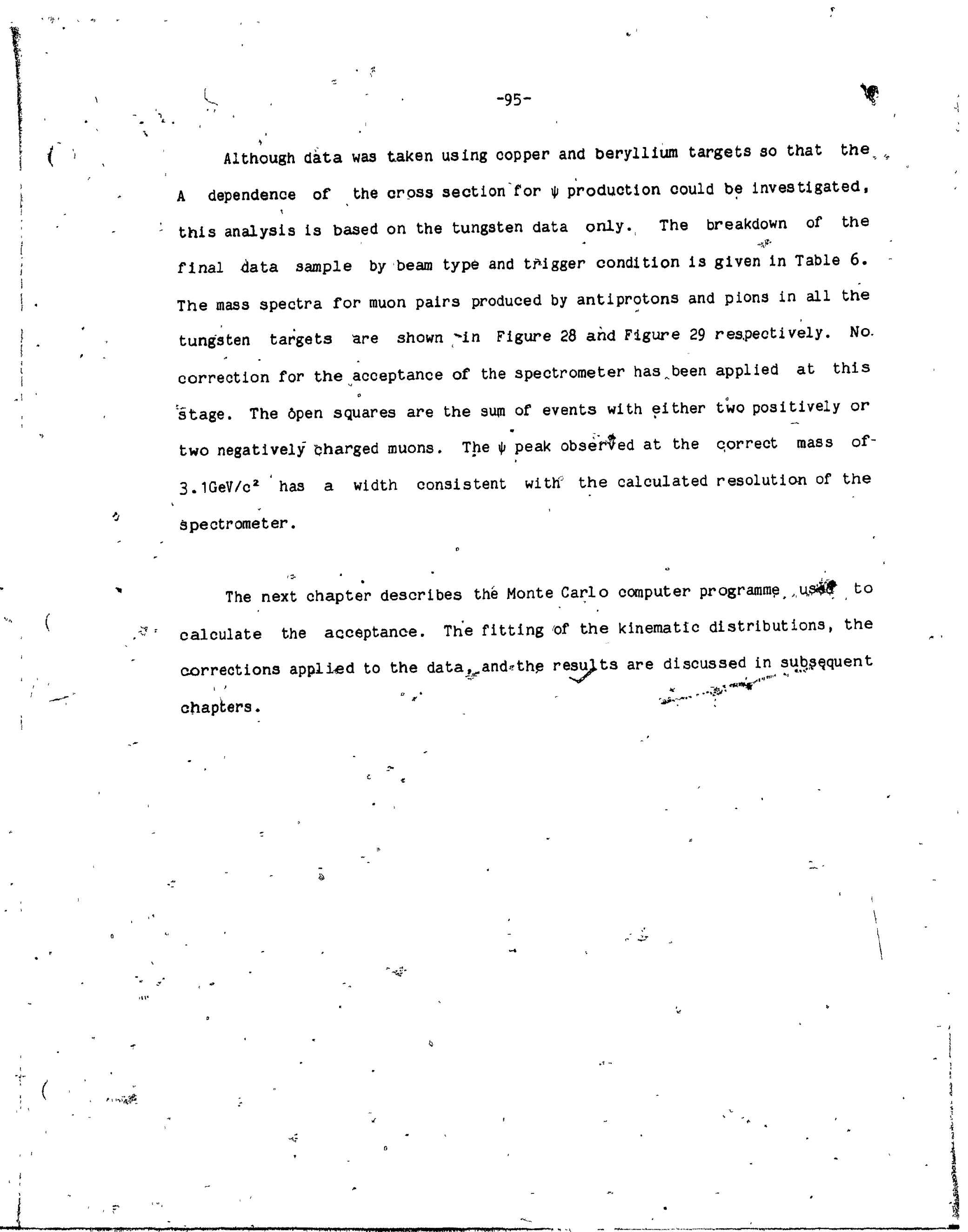


This table summarizes the number of reconstructed high mass muon pairs with $\rightarrow \quad M$ between 4 and $9 \mathrm{GeV} / \mathrm{c}^{2}$ and $\mathrm{x}_{\mathrm{F}} \geq 0$. Oppositely charged and like charged pairs are listed in separate columins.

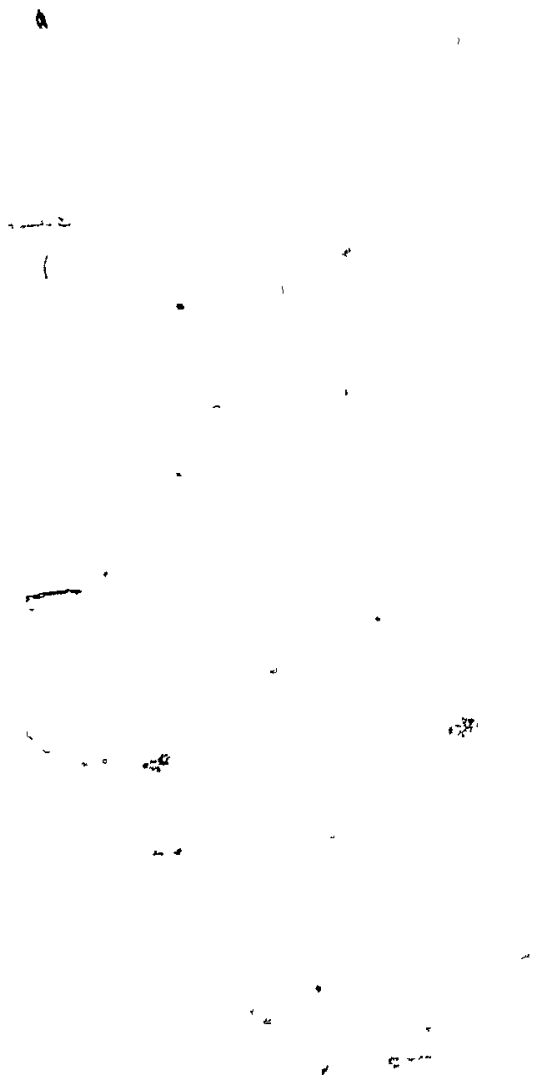




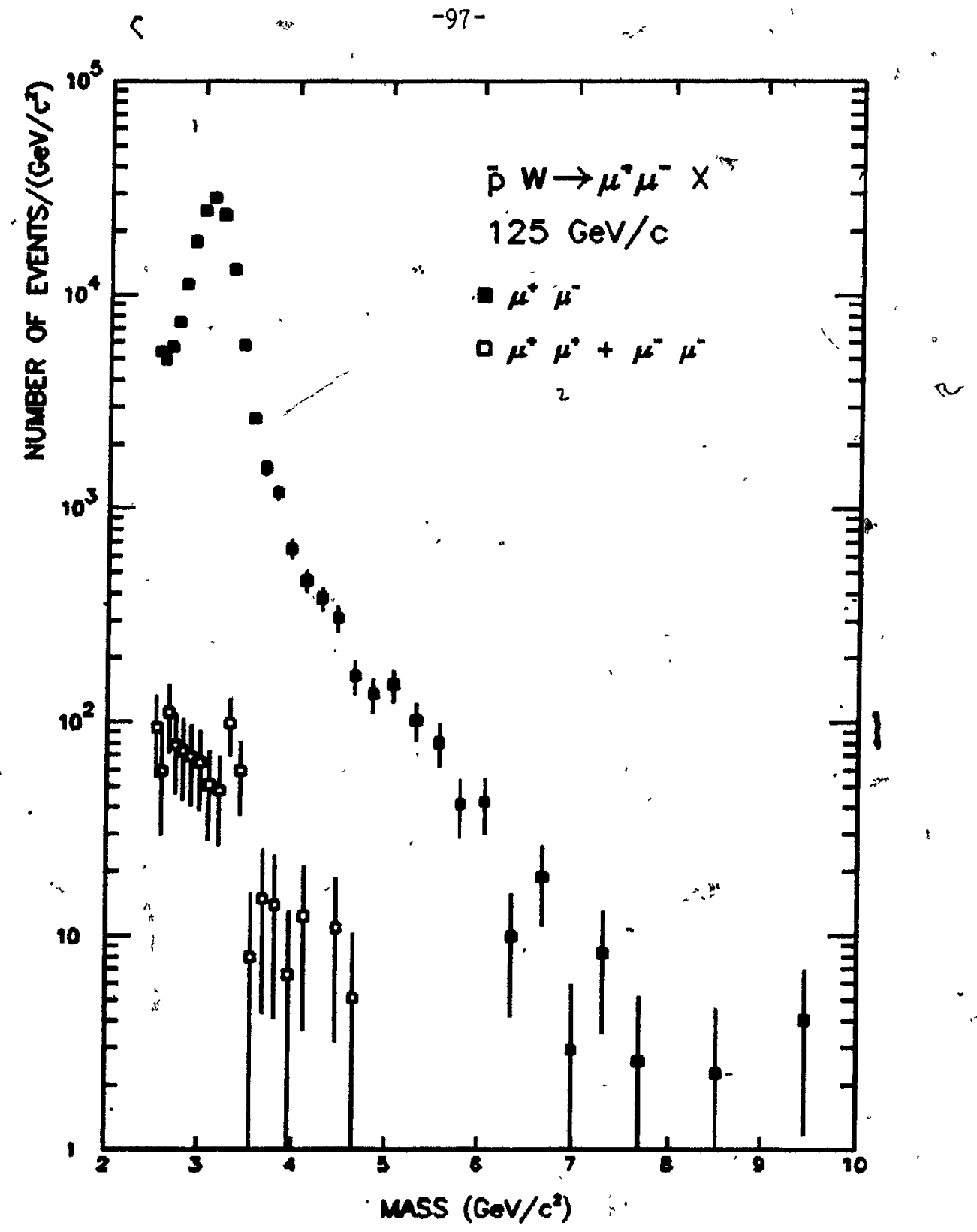

Figure 28 - Uncorrected Antiproton Produced Mass Spectrum

The solid squares show the number of oppositely charged muon pairs produced in antiproton-tungsten collisions as a function of mass. The open squares show the sum of pairs of positively charged muons and pairs of negatively charged muons. 


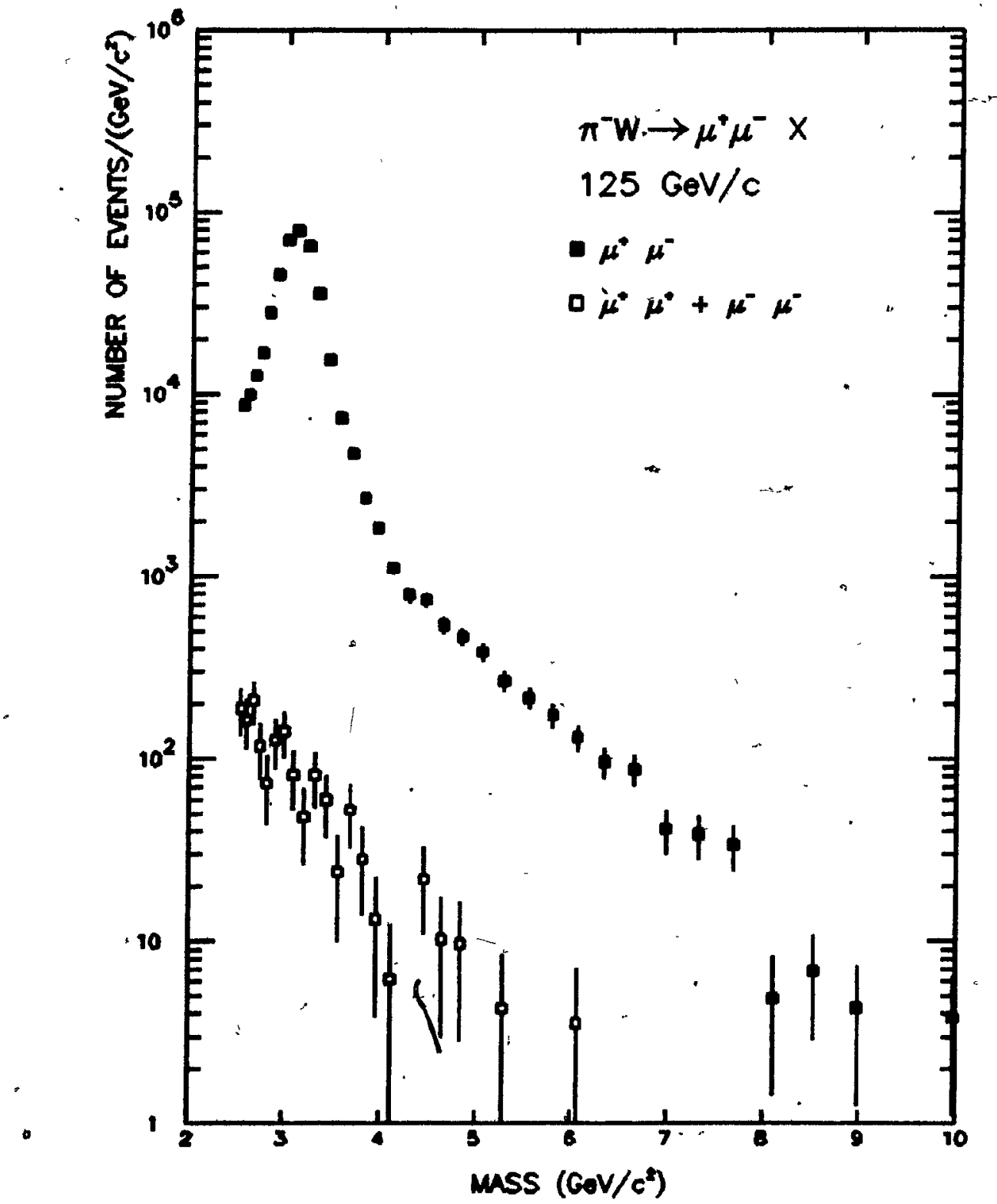

Figure 29 - Uncorrected Pion Produced Mass Spectrum

The solid squares show the number of oppositely charged muion pairs produced in pion-tungsten collision's as a function of mass. The onen squares show the sum of pairs of positively charged muons and pairs of negatively charged muons. 


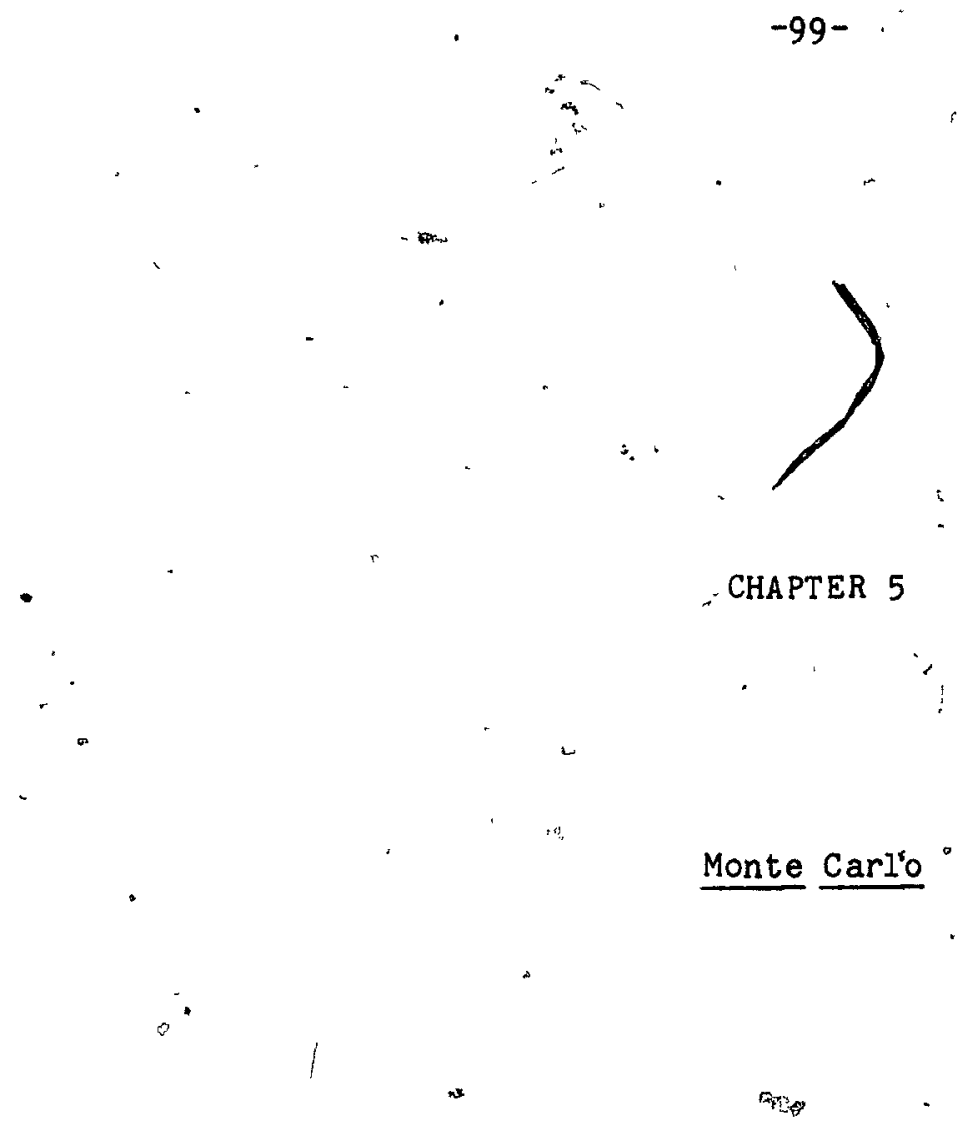

The largest correction that had to be made to the data was. for the limited acceptance of - the spectrometer itself. A FORTRAN Monte Carlo programme was written for the Fermilab Cyber 175 computer [86] to "model the apparatus and calculate the acceptance.

$\therefore \rightarrow \cdots$

The programme sought to simulate the appàratus as closely as possible. Events were generated randomly throughout phase space using the measured beam ęnergy spectra and profiles, and thd, resulting pairs of muons were propagated through the spectrometer taking into account multiple scattering, energy loss, and the Fermi motion of the target nucleon. The track positions at the chambers were digitized, the counters hit were recorded, and the results were written to a disk file in the same format as the data events. Background hits were included as despribed in the last chapter to allow for electronics dead time and give the same pattern recognition 

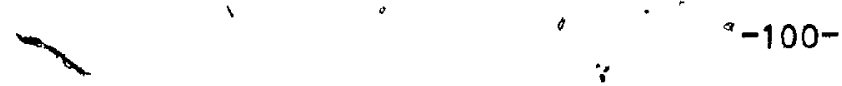

efficiency as for the data events. The events were subject to the same set of analysis programmes and the same trigger and aperture requirements as the data events. Both, the generated and reconstructed kinematic variables of each Montẹ Carlo event were stored on the disk file.

This chapter describes the deţails of the simulation programme itself and the mechanics associated with reweighting events. The list of events generated summarized all of thel information about the acceptance/of the spectrometer and it could pe read and reweighted by the fitting programmes, as described in the next, chapter, without repeating the entire Monte Carlo calculation.

This Monte Carlo programme was compared to an independently written programme[89]入 Each programme generated 1.0 × $10^{5}$ antiproton,produced muon' pairs. using the kinematic distributions given below. Tho programmes were found to agree on both the absolute values and shapés of the acceptance as a function of the kinematic variables to within statistical errors.

\subsection{Beam Particle}

The four, vector of the beam particle-was selected from files of beam momenta taken from the data summary tapes to give the correct beam profile and momentum distribution in the Monte Carlo. The files contained approximately 9000 and 15000 events for the $1981^{\circ}$ and 1982 data runs respectively.

\section{(2)}




\subsection{Target Particle} .

The four vector of the target nucleon was generated according to a simple Fermi gas model[90] to take into account the motion of the nucleons inside the heavy target. The target nucleon was given an isotropic angular distribution in the laboratory frame with momentum distributed between 0 and the Fermi momentum as follows:

t. $\frac{d N}{d p}=3 p^{2} / p_{\text {Fermi }}^{3}$.

The Fermi momentum for the various targets was taken as[91][92][93]

$$
\begin{aligned}
\mathrm{p}_{\mathrm{Fermi}}(\mathrm{Be}) & =0.203 \mathrm{GeV} / \mathrm{c}, \\
\mathrm{p}_{\mathrm{Ferm1}}(\mathrm{Cu}) & =0.250 \mathrm{GeV} / \mathrm{c} \text {, and }
\end{aligned}
$$

\section{I}

$$
p_{\text {Ferm } 1}(W)=0.265 \mathrm{GeV} / \mathrm{c} \text {. }
$$

The effect of including nigh momentum tails[94] in" the Fermi distribution was investigated in the simulation and found not to be significant.

\subsection{Event Generation}

For each muon pair in the continuum region, the five kinematic variables and a random azimuthal" angle about the beam direction were generated according to the following unnormalized distributions in the beam-target centre of mass system:

$$
\begin{aligned}
& \frac{d N}{d M}=\exp \left(-\alpha_{M} M\right), \\
& \frac{d N}{d x_{F}}=\exp \left(-\frac{1}{2}\left(\frac{x_{F}-x_{F o}}{a_{x_{F}}}\right)^{2}\right), \\
& \frac{d N}{d p_{T}}=p_{T}\left[1+\left(\frac{p_{T}}{p_{T o}}\right)^{2}\right]^{\alpha_{1}}, \\
& \frac{d N}{d \cos \theta}=1+\lambda \cos ^{2} \theta, \text { and }
\end{aligned}
$$




$$
\frac{\mathrm{dN}}{\mathrm{d} \phi}=1 \text {. }
$$

The parameters used in the distributions were taken from a preliminary analysis of the data[95] and are as follaws:

$$
\alpha_{M}=1.26
$$$$
x_{F_{0}}=0.0 \text {, }
$$$$
{ }^{0} x_{F}=0.27
$$$$
\mathrm{p}_{\mathrm{T} \mathrm{i}_{\mathrm{i}}}=3.1,
$$

$$
a_{p_{T}}=-10 \cdot 2 \text {, and }
$$

$$
\lambda=1.0
$$

for ant1proton produced pairs; and

$$
\begin{aligned}
& \alpha_{M}=1.0 \\
& x_{F_{0}}=0.0
\end{aligned}
$$$$
{ }^{0} x_{F}=0.37 \text {, }
$$$$
\mathrm{p}_{\mathrm{T} 0}=3.1
$$

$$
\therefore \quad \alpha_{p_{T}}^{*}=-9 \cdot 3 r \text { and }
$$

$\therefore, \lambda=i .0$

for pion produced palrs.

,To minimize computer time, the mass and $\mathrm{p}_{\mathrm{T}}^{*}$ of the muon pair were calculated by integrating the probability distributions and inverting the equations to $f$ ind $M$ and $p_{T}$ in terms of the cumulative probabilities as idescribed by James in his review. of "Monte Carlo techniques[96]. For example, the mass distribution normalized to unit probability between $M_{\text {min }}$ and $M_{\max }$ is given by 
$\because \quad d P=\alpha_{M} \cdot \exp \left(a_{M}\left(M_{m i n}-M\right)\right) \cdot d M \cdot\left[1-\exp \left(\alpha_{M}\left(M_{m i n}-M_{m a x}\right)\right)\right]^{-1}$.
Defining the umulative probability distribution to be $y(M)=\int_{M}^{\dot{M}_{\max }} d P$.

and inverting toin obtain $M$ in terms of $y$, we $f$ ind

$M=M_{\min }-\frac{1}{\alpha_{M}} \ln \left[1-y \cdot \exp \left(\alpha_{M}\left(M_{\min }-M_{\max }\right)\right)\right]$.

The random number generator, RANF[97], was used to give a value of $y$ uniformly distributed between 0 and 1 , and the mass. was then calculated from the. equation above. The $x_{F}$ distribution was obtained by scafing a normally distributed random number generated by the CERN libraty programme NORRAN[98][99]. Because the inversion of the cumulative probablility of the $\cos \theta$ distribution involves the solution of a cubic equation, the standard tèchnique of generating a random value for $\cos \theta$ between \pm 1 but using it only if a secopro random number between 0 and 1 was less than $\left[1+\cos ^{2} \theta\right] / 2$, was usèd instead.

The kinematic quantitiès were used to caloulatè the four vectors of the two muons in the beam-target centre of mass frame. The CERN library routine LORENC[100] was used to transform the beam and target four momenta from the laboratory frame to the beam-target centre of mass frame $\left\{\right.$ The values of $x_{F}$, $\mathrm{p}_{\mathrm{T}}$, and the azimuthal angle of the pair about the beam direction were used to calculate the motion of the muon pair in the centre of mass frame. The beam and target particles were transformed to the muon palr rest frame and used to calculate the $X, Y$ and $Z$ axes of the Gottfreld-Jackson frame. A unit vector along the $Z$ axis was scaled ta-jive a muon energy of $M / 2$ in the rest frame of the pair. The vector was rotated by an angle of $\theta$ about the $y$ axis and then by an angle of $\phi$ about the $z$ axis to give the three momentum vector of the positive muon in the rest frame of the pair. The three momentum of the negative muon was taken as equal and opposite. The muon 
, where $\psi$ is the dimensionless ratio

a.

$$
\psi=Y / Z \text {, and }
$$

the projected RMS scattering angle is given by [13].

$$
\theta_{\text {YRMS }}=\frac{0.015}{-\beta \mathrm{P}} \sqrt{Z_{\mathrm{Zad}}}
$$

and

$\beta$ is the velocity of the particle in units of $c$,

$\therefore p$ is the momentum of the track in $\mathrm{GeV} / \mathrm{c}$,

$Z$ is the length of the scatterer, and

$Z_{\text {rad }}$ is the radiation length [13] for the particular target material. A similar expression also holds for the $\mathrm{X}-\mathrm{Z}$ plane.

The muon momenta in the laboratory frame were also correated for energy loss in the target using tables[102][103] calculated from the Bethe-Bloch Ionization formula with corrections for density effect, brensstrahlung, and nuclear interactions. The tables of energy loss for tungsten, copper, berylifum, iron and concrete were parameterized for kinetic energies between $100 \mathrm{MeV} / \mathrm{c}$ and $125 \mathrm{GeV} / \mathrm{c}$, and these are shown in Figure 30. The' po MeVlo and parameterization for iron was compared with other references in the literature[104][88] and found to agree to better than 1 percent for muon energies in the range of interest.

The muons were also propagated through the beam dump taking into docount scattering and energy foss as in the target. The track coordinates at the absorber chamber were recorded for use in the redonstruction. 


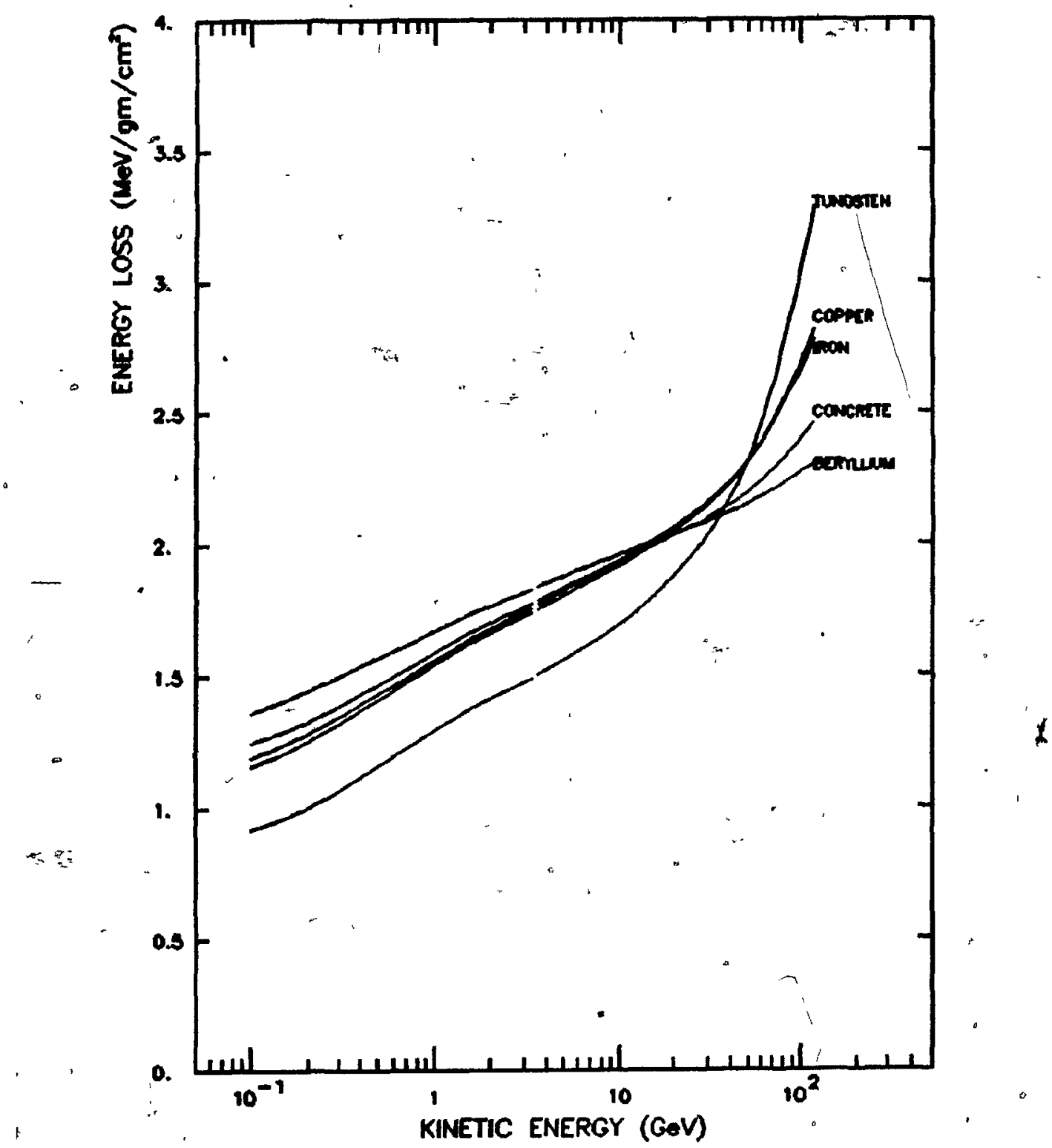

Figure 30 - Energy Loss for Muons

The energy loss for muons in the various materials used in the spectrometer is shown as a function of the kinetic energy of the muon. 


\subsection{Spectrometer}

Muons emerging from the dump were required to pass through regions, corresponding to the physical apertures of the front chambers. The trajectories of both muons were examined to determine whether either muon had passed through an absorber counter quadrant. If both muons passed within all of the physical apertures upstream of the magnet, the particles were propagated through the magnetic field using a square field of effective length $2.03 \mathrm{~m}$ and central field value 13.6 Kgauss. The tracks were also required to pass through apertures corresponding to the physical dimensions of the magnet field region. Downstream of the magnet, the tracks were checked to make sure that they passed through the drift chambers and CPX and CPY hodoscope counter arrays.

5.6 Muan Hodoscopes and Trigger ...

Muons tracks passing through all the apertures of the spectraneter were propagated through the muon filter walls taking into account energy loss and multiple scattering as had been done in the target and beam dump. Tracks were required to hit apertures corresponding to the muon hodoscope counter arrays. Counters hit by the tracks were combined to form triple coincidence channels identical to the corresponding hardware channels. The information from these triple coincidences was then combined with the absorber counter information to decide whether the event should be rejected, accepted by the level 1 trigger, or accepted by the lewel 2 trigger. 


\subsection{Trigger Processor}

The trigger processor algorithm was applied ${ }^{\circ}$ to all events accepted by the software simulation of the apparatus. The track coordinates in the mrear drift chambers were digitized and used to calculate the projected slope, $d x / d z$, downstream of the magnet. The track positions at the CPY hodoscope were used to give the slope $\mathrm{dY} / \mathrm{dZ}$. The downstream track was projected to the magnet bend plane and used to determine the muon momentum assuming that the production vertex was at the centre of the target. The mass of the muon pair was calculated using the approximation $p^{M^{2}}=p_{1} p_{2} \theta^{2}$

where $p_{2}$ and $p_{2}$ are the individual muon momenta and ' $d$ is the opening angle between them in the laboratory frame. Only events reconstructing to a mass of greater than $2.0 \mathrm{GeV} / \mathrm{c}^{2}$ were accepted.

\subsection{Reconstruction}

:

To take into account any dependence of the reconstruction efficiency on the kinematic variables of the muon pairs, all of the Monte Carlo events were reconstructed by the analysis programmes used for the real data as described in the last chapter. Events accepted by the sof tware model of the spectrometer were digitized and recorded on a disk fle in the original data format. Drift chamber hits from events triggered by beam partzcles were used to simulate background hitsi in the chambers. Events from the same beam runs were, also used to calculate the inefficiency of the chambers resulting from the dead time in the elèctronics. An efficiency per plane of 99.5 percent was used for the drift chambers and the efectronics dead time was 
taken to be $300 \mathrm{~ns}$. The Monte Carlo events were processed by the complete set of analysis programmes and a list of generated and reconstructed kinematio variables was stored on tape for use by the fitting programme.

\subsection{Reweighting Events

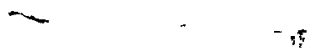

The calculation of the accèptance using the simulation programme can be viewed as the approximation of an integral by Monte Carlo methods. The reweighting of Monte Carlo events is then equivalent to using the specific technique of importance sampling. Economic considerations require the reweighting of events. The justifications for Monte Carlo evaluation of integrals, comparisons with other methods of numerical integration, importance sampling, and the attendant errors are discussed by James[96], in his review of Monte Carlo methods, and in a book by Schreider [105].

As discussed previously, the Monte Carla events were generated assuming that the muon pairs were distributed in phase space according to a -multidimensional distribution; $G(X)$, where

$$
X=\left(M, x_{F}, p_{T}, \ldots\right)
$$

is the set of kinematic variables, and can be considered to include other parameters such - as the azimuthal angle about the beam, multiple scattering anǵles, and nucléon momenta due to Fermi motion. For a given event. $x$, the acceptance is either' 1 or 0 ; the event is ei ther accepted or it is rejected. The dalculation of the overall acceptance of the apparatus is equivalent to 11 " The

the evaluation of an integral, that is,

$$
I=\int A(X) G(X) d X \text {, }
$$

where $\dot{G}(x)$ is normalized such that

$$
\text { - } \int G(x) d x=1
$$


The evalution is accomplished by making the approximation

$$
\int \mathcal{G}(X) A(X) d X=\frac{1}{N} \sum_{i=1}^{N} A\left(X_{i} \rho,\right.
$$

where the ' $x_{i} ;$ are $N$ points randomly distributed according to the multidimensional distribution $G(X)$.

As will become clear in the next chaptér, it is necessary to be able to. aluate the acceptance for other multidimensional distributions such as $\bar{F}(\bar{X})$. It is possible to repeat the simulation with the new distributions, but the generation and tracking of the events is an expensive process requiring substantial amounts of computer, time. A more ecorionical. alternative' is to' 'run the simulation once using an'intal distribution, $G(X)$, and record a list of the kinematic variables for each event "and whether or not the everit was accepted. The acceptance 'can then'be evaluated for any other distribution, $F(X)$, normalized such that.

$$
\int F(X) d X=1 ;:
$$

, by assigning each event a weight of $F^{2}\left(X_{i}\right) / G\left(X_{1}\right)$. This gives for the new distribution

$$
I^{\prime}=\int F(X) A(X) d X=i_{N}^{1} \cdot \sum_{i=1}^{N} A\left(X_{1}\right) F\left(X_{i}\right) / G\left(X_{i}\right)
$$

. where the $\dot{x}_{i}$ are the same $N$ points, randomly distributed according to the multidimensional distribution $G(X)$.

5.10 'Acceptảnce.

The data must be corrected for the acceptance of the spectrometer, but the acceptance in turn depends on the kinematic distributions of the data. The final acceptance of the spectrameter was calculated usting the 'results from. fits descmbed in the next chapter. The acceptance for high mass muon pairs produced by antiprotons as a function of the kinematic varlables is 

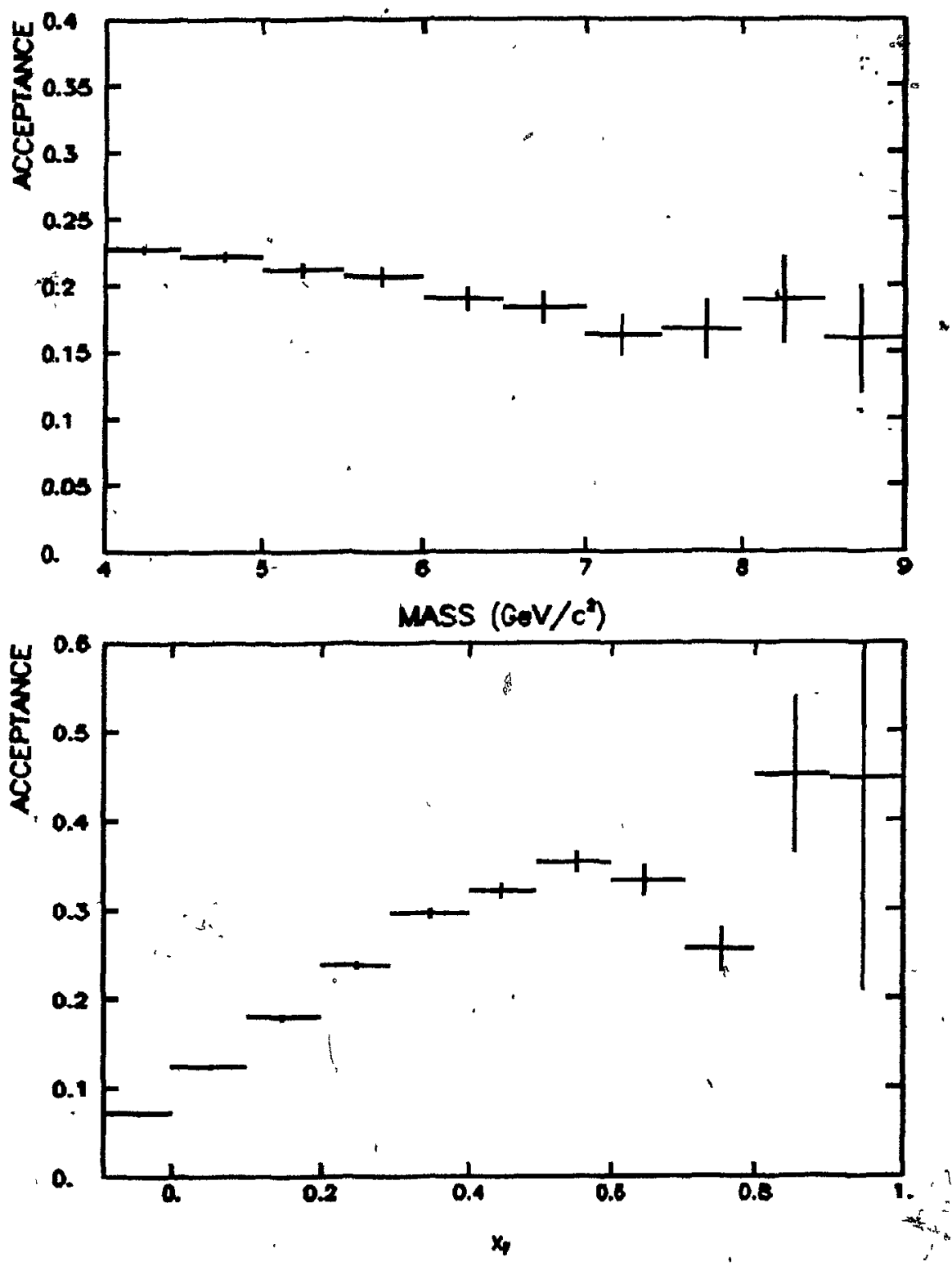

Figure 31 - Acceptance vs Mass and $x_{F}$ for Antiprotons

The upper figure shows the acceptance of the spectrometer for high mass muon pairs produced in antiproton-tungsten collisions as a function of the invariant mass of the pair. The lower figure shows the acceptance as a function of the longitudinal momentum of the pair, expressed in terms, of the dimensionless variable $x_{F}$. 

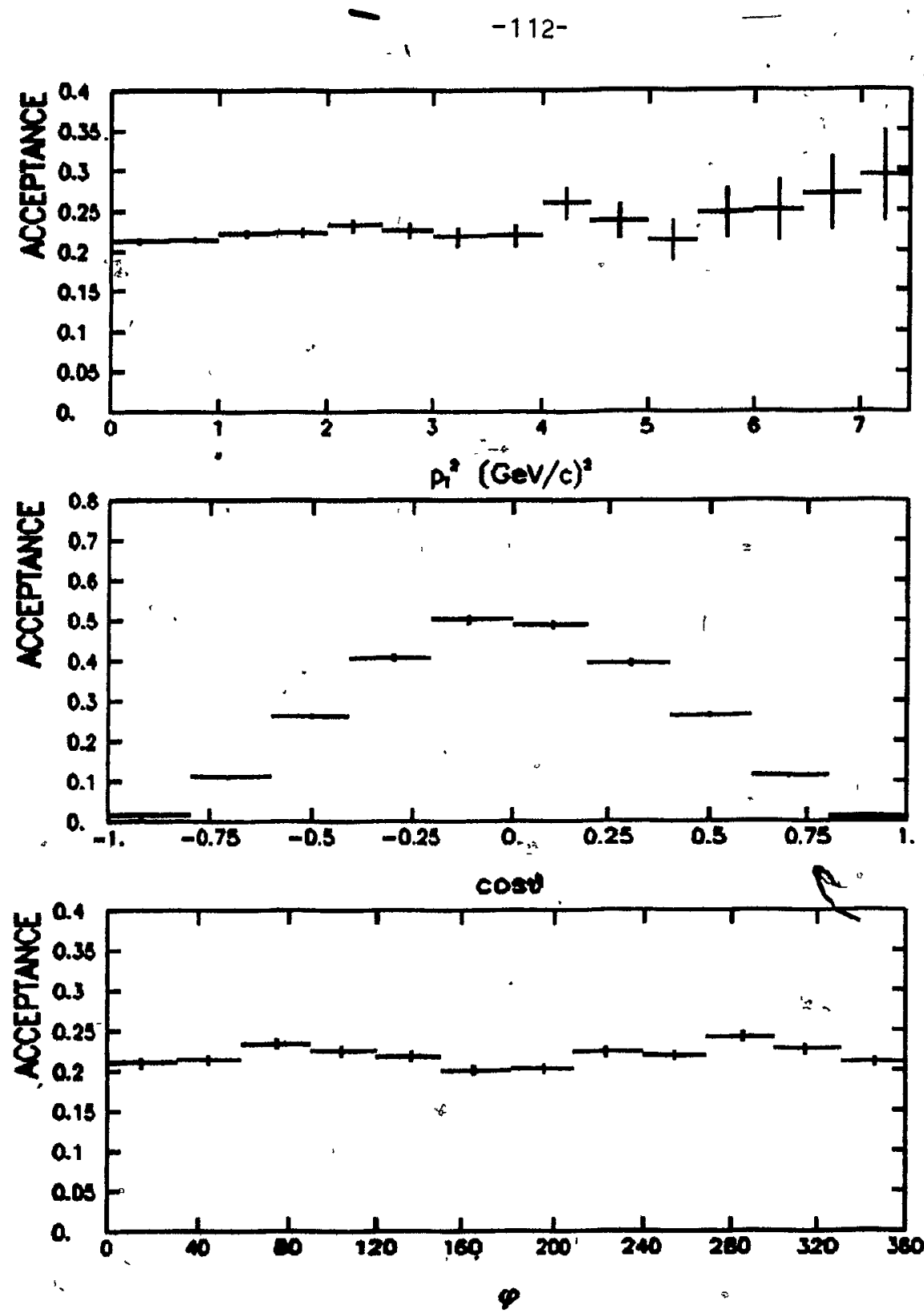

Figure 32 - Acceptance vs $\mathrm{p}_{\mathrm{T}}{ }^{2}, \cos \theta$ and $\phi$ for Antiprotons

The upper figure shows the acceptance of the spectrometer for high mass muon pairs produced in antiproton-tungsten tungsten nucleus collisions as a function of the transverse momentum of the pair. The central and lower figures show the acceptance as a function of the decay parameters $\cos \theta$ and $\phi$ respectively. 
shown in Figure 31 and Figure 32 and the acceptance for pion produced pairs is shown in Figure 33 and Figure 34 . Note that the overall acceptance is typically 20 percent for both antiproton and pion produced pairs. The acceptance does not vary rapidly for most of the range of the kinematic variables but does fall sharply at high values of $\cos ^{2} \theta$ and for negative values of $x_{F}$. In both of these cases, one of the muons of the pair did not have enough energy in the laboratory frame to traverse the entire apparatus, since a muon required an inital momentum of $6 \mathrm{GeV} / \mathrm{c}$ to penetrate to the final muon counter hodoscope. The acceptance for pion produced pairs also falls of at very high $x_{F}$ because these events were required to satisfy more. stringent trigger requirements during most of the data taking. 


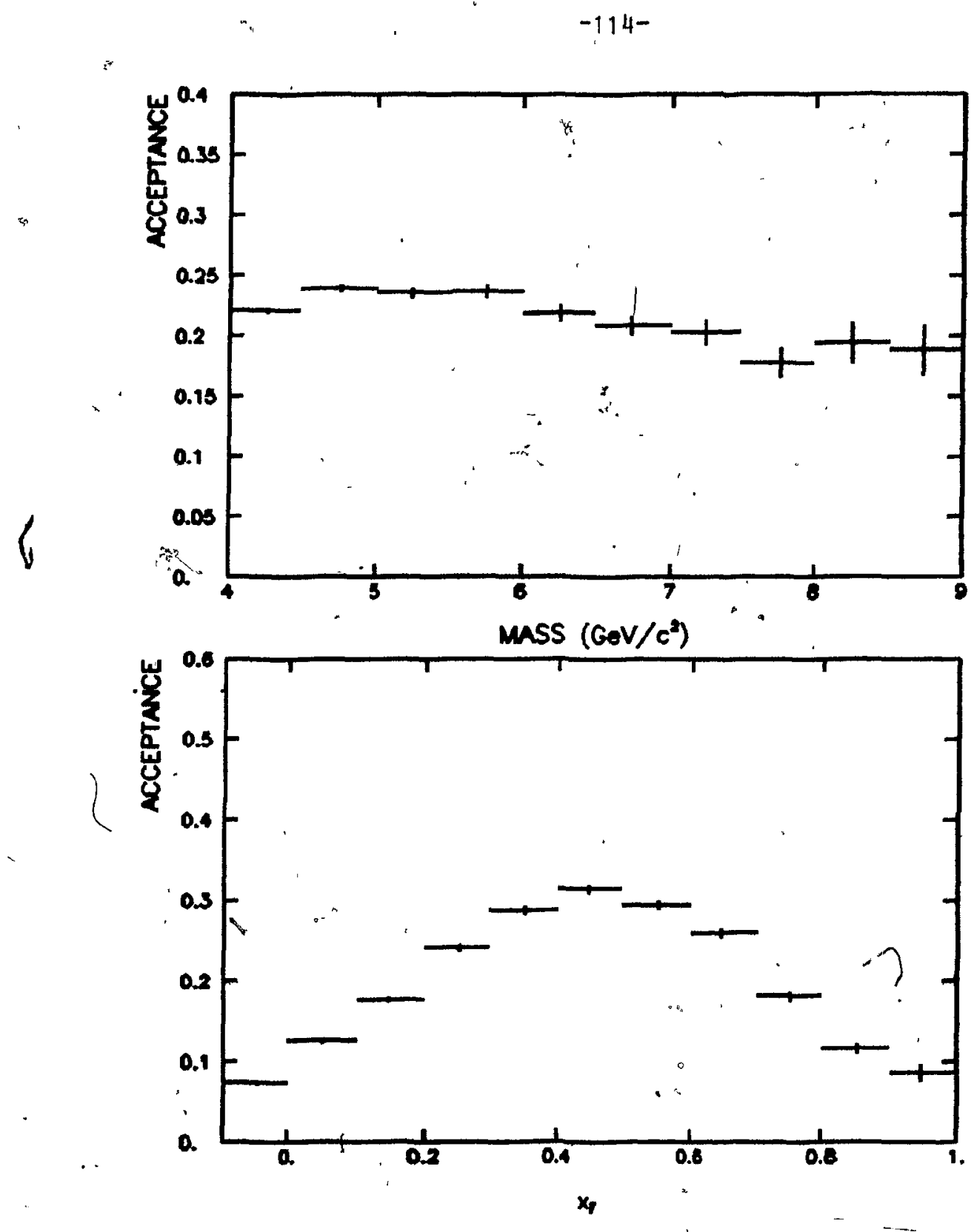

Flgure 33 - Acceptance vs Mass and $x_{F}$ for $P_{10 n s}$

The upper figure shows the acceptance of the spectrometer for high mass muon pairs produced in piton-tungsten nucleus collisions as a function of the invarlant mass of the pair. The lower figure shows the acceptance as a function of the longitudinal momentum of the pair, expressed in terms of the dimensionless variable $x_{F}$. 

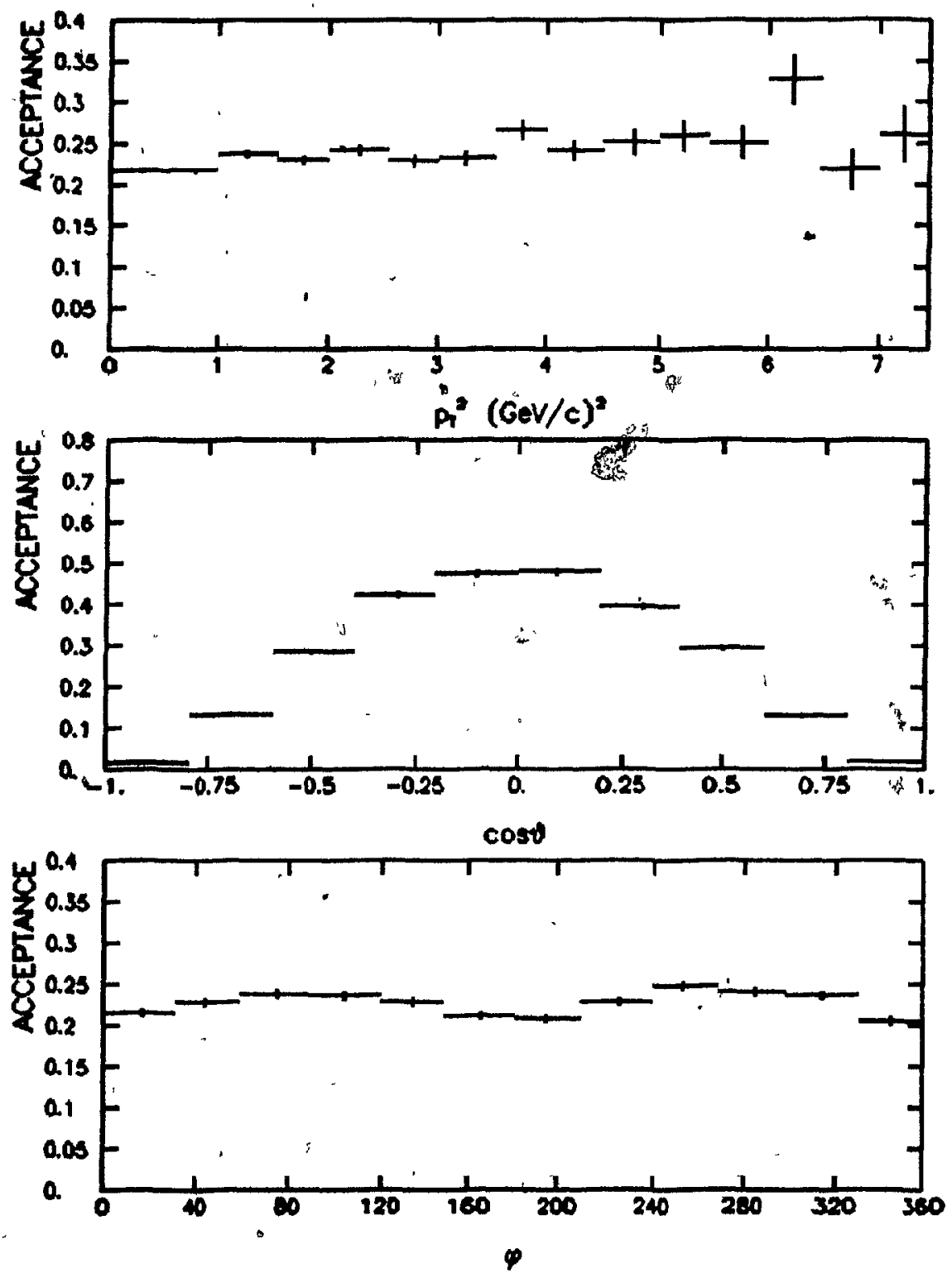

Figure 34 - Acceptance vs $p_{\mathrm{T}}^{2}, \cos \theta$ and $\phi$ for Pions

The upper figure shows the acceptance of the spectrometer for high mass. muon pairs produced in pion-tungsten nucleus collisions as a function of the transverse momentum of the pair. The central and lower figures show the acceptance as a function of the decay parameters $\cos \theta$ nd $\phi$ respectively. 
CHAPTER 6

Analysis

Ideally the cross section per nucleon can be obtained by dividing the number of events observed by the number of beam particles and normalizing to the effective number of nucleons per $\mathrm{cm}^{2}$ in the target. In practice, the apparatus is notyone hundred percent efficient and has limited acceptance. An accidental coi fidence between a halo particle and a muon from hadron decay can mimi a muor pair. In thick targets, secondary hadrons can interact" and produce real muon pairs. Corrections must be applied for all of these effects.

- The reconstruction of the kinematic variables of the muon pair events was discussed in.chapter 4. A list of the varlables for each reconstructed event was stored on a disk file by the analysis programmes. The generation of a similar list of Monte Carlo events, summarizing the complete knowledge of the acceptance of the apparatus, was discussed in the last chapter. The 
maximum likelihood method was used to fit the unbinned data events to empirical parameterizations of the kinematic distributions, and the acceptance of the spectroneter was recalculated at each step of the fit by rewelghting the list of Monte Carlo events. The contamination of the dafta sample by accidental colncidences between unçorrelated muons was evaluateq by examining the number, of positively and negatively charged muon pairs. A correction for this background was made by subtracting the number of like charged pairs from the number of oppositely charged palrs on a bin by bin basis. The contamination of the data sample by muon pairs produced by reinteracting secondary" particles was determined by examining the $\psi$ "cross section as a function of the length of the target. A correction for contamination of the high mass region by muon pairs produced in the decay of the ' $\psi^{\prime}$ resonance was also applifed to the tofal cross section. Corrections. were made to the beam flux to take into account any confusion caused by muitiple beam particles in the same RF beam bucket.

These corrections and any resulting systematic uncertainties are discussed in this chapter. The final cross sections and kinematic distributions are presented in the next chapter.

6.1 Fitting and Acceptance

A common approach to multidimensional fitting in particle physics is the fitting of the data in projection[106j. The experimental apparatus as a rule has limited acceptance in some regions of phase space. The parent distributions of the data pointis can only be determined as well as the acceptance is known, but the acceptance in turn depends on the parent distributions of the data. The data points are binned separately in each of 
the variables, that is, projected onto the axes of the multidimensional space to obtain a number of one or two dimensional distributions. The acceptance is calculated for each of the projections using some initial guess for the parent distributions: The one dimensional projections are then corrected for 'acceptance and fit to the same parameterizations using the least squares method. The results of the fit are used to recalculate the acceptance and the process is repeated until there is no difference between the parameters used to caiculate the adceptance and the parameters obtained from the fit.

In principle, it is possible to bin the data points multidimensionally and fit them using the least squares method, but the number of bins that must be used grows rapldly when the number of dimensions exceeds two or. three; "The least squares method does not handre bins with 0 or 1 event. gracefully; this is a problem that occurs when a small number of events are binned in a largé number of dimensidns. Binning events also results in a loss of information. Since the maximum likelihood method can work with the unbinned data, all of the information is used. If the data is not binned, no problems can arise because bins contain small numbers of events. In the asymptotic limit, as the number of data points to be fit approaches infinity, parameter estimates from the maximum likelihood method are unblased and normally distributed with minimum variance, or, in other words, asymptotically no other method can do better.

The maximum 1 ikelihood method is not without disadvantages.-In general l the maximum likelihood 'method is more expensive (uses more computer time) than other methods.- The asymptotic propertles do not necessarily nold for finite numbers of events. As Eadie[107] has pointed out, the fact that all 
of the information is used does not imply that it is necessarily used in the best way. On the whole, however, the maximum 11 kelihood method still . provides the best "way to extract information from a limited number of events. One advantage over the usual approach of fitting in projection that should not be underestimated is the presence of an objective convergence criteria. Computing stops when the likelthoo function has been maximized and not when the experimenter feels that nothing is to be gained by $f$ urther iteration.

Tofit the data points. using the maximum likel inood method, the individual kinematic distributions were parameterized using simple functional forms which were multiplied together to obtain a multidimensional distribution. The multidimensional distribution was used to give the probability of observing a set of data events as a funçtion of the parameters. All of the distributions were fit simultaneously by finding the set of parameters which, 'maximized the probability of observing the experimental data points. The acceptance was recalculated at each step of the fit by reweighting the ilist of Mante Carlo events, as discussed in the last chapter. The maximum likel thood method is treated in some detall in the books by Eadie[107], and by Frodesen[108] and will only be outlined here.

The mass distribution was parameterized with a falling exponential which was normalized to unity between $M_{\min }$ and $M_{\max }$, that is,

$$
P(M)=\alpha_{M} \exp \left(-\alpha_{M} M\right) \cdot\left[\exp \left(-\alpha_{M} M_{\min }\right)-\exp \left(-\alpha_{M} M_{\max }\right)\right]^{-1}
$$

A Johnson bounded empirical distribution[109], a transformation of a Gaussian designed to fall of to zero at the kinematic limits of \pm 1 , was used for the $x_{F}$ distribution:

$$
P\left(x_{F}^{\prime}\right)=\sqrt{ } \frac{2}{\pi} \exp \left(-\frac{1}{2} z^{2}\right) \frac{d z}{d x_{F}}
$$


where

$$
z=\frac{1}{\sigma_{X_{F}}}\left[\ln \left(\frac{x_{F}+1}{1-x_{F}}\right)-\ln \left(\frac{x_{F}+1}{1-x_{\hat{F} 0}}\right)\right]
$$

When normalized to uni ty between $x_{F m i n}$ and 1 , this distribution becomes

$$
\left.P\left(x_{F} \mid x_{F o}, o_{x_{F}}\right)=2 \sqrt{ } \frac{2}{\pi} \exp \left(-\frac{1}{2} z^{2}\right) \cdot\left[0_{x_{F}}\left(1-\operatorname{erf}\left(z_{m i n}\right)\right)\left(x_{F}+1\right)\left(1-x_{F}\right)\right)\right]^{-1}
$$

A Gaussian, normalized to unity between 0 and $\infty$, was chosen, for the $\mathrm{p}_{\mathrm{T}}{ }^{2}$ distribution:

$$
\mathrm{P}\left(\mathrm{p}_{\mathrm{T}^{2}} \mid \mathrm{p}_{\mathrm{T} 0}\right)=\frac{1}{\mathrm{p}_{\mathrm{T} 0}} \sqrt{\frac{2}{\pi}} \exp \left(-\frac{1}{2}\left(\frac{\mathrm{p}_{\mathrm{T}}}{\mathrm{p}_{\mathrm{T} 0}}\right)^{2}\right) .
$$

In terms of- $\mathrm{p}_{\mathrm{T}}$ this becomes:

$$
P\left(P_{T} \mid p_{T 0}\right)=2\left(\frac{p_{T}}{p_{T 0}}\right) \sqrt{ } \frac{2}{\pi} \exp \left(-\frac{1}{2}\left(\frac{p_{T}}{p_{T 0}}\right)^{2}\right) \text {. }
$$

The $\cos \theta$ distribution was taken to be

$$
P(\cos \theta \mid \lambda)=\frac{1}{2[1+\lambda / 3]}\left[1+\lambda \cos ^{2} \theta\right] \text {. }
$$

The $\phi$ distribution was assumed to be constant, that is,

$$
P(\phi)=\frac{1}{2 \pi}
$$

as was the distribution of the events in the azimuthal angle about the beam direction.

To the extent that these distributions are flexible enough to represent the underlying parent distributions, the likelihood or probability that the $i^{\text {th }}$ event will have the kinematic varlables

$$
x_{i}=\left(M_{1}, x_{F i}, p_{T} i, \cos \theta_{i}, \phi_{i}\right),
$$

is

$$
L\left(x_{1} \mid \Gamma\right)=P\left(X_{1} \mid \Gamma\right) A\left(X_{1}\right)\left[\int P(X \mid \Gamma) A(x) d x\right]^{-1}
$$

where

$$
P\left(x_{i} \mid \dot{\Gamma}\right)=P\left(M_{i} \mid \alpha_{M}\right) P\left(x_{F i} \mid x_{F 0, \sigma_{x_{F}}}\right) P\left(p_{T i} \mid \sigma_{p_{T}}\right) P\left(\cos \dot{\theta}_{i} \mid \lambda\right)
$$

is the probability that an event will be produced with kinematic variables $x_{i}$. The elements of $\Gamma$,

$$
\Gamma=\left(\alpha_{M}, x_{F}, \sigma_{x_{F}}, \sigma_{p_{T}}, \lambda\right) \text {, }
$$

are the parameters of the kinematic distributions and $A\left(X_{1}\right)$ is the 
acceptance of the spectrometer for the event $x_{i}$. By definition the acceptance is 1 for an event that has been accepted.

It follows that the likel ihood of observing a set of $N$ events $X=\left(X_{0}\right.$, $\mathrm{X}_{1}, \ldots \mathrm{x}_{\mathrm{N}}>1 \mathrm{~s}$

$L(X \mid \Gamma)=\pi_{i=1}^{N} L^{\prime}\left(X_{i} \mid \Gamma\right)$.

Fitting the $N$ data points to the multidimensional distribution $P(X \mid \Gamma)$ with the maximum likelihood method involves finding the set of parameters $\Gamma$. which give the maximum value of the likelihood function, that is, the highest probability that $N$ actual data points would be observed. In practice, because the logarithm of the iikelihood is better behaved, the negative logarithm of the likelihood function is minimized, rather than the -ikel ihood itself maximized.

1

The integral in the denominator of the likelinood function was evaluated using the list of Monte Carlo events. As discussed in the last chapter, the simulation programme had been used to generate muon pairs and follow them through the apparatus. The events were generated randomly throughout phase space according to a multidimensional distribution, $G(X)$, and listed on a disk file. The integral in the denominator of the" likelihood function can then be evaluated as.

$$
\int P(X \mid \Gamma) A(X) d X=\frac{1}{N} \sum P\left(X_{1} \mid \Gamma\right) A\left(X_{i}\right) / G\left(X_{1}\right),
$$

where

$\mathrm{N}$ is the number of events generated, $P(X \mid r)$ is the multidimensional distribution being fit to the data points,

$A(X)$ is the acceptance for an event with kinematic variables. $X$, that 1s, 1 if the event is accepted and 0 if it is not, and 
$\overline{G(X)}$ is the multidimensional distribution used to generate the Monte Carlo events.

A FORTRAN subroutine was written which evaluated the negative logarithm of the likelihood for a given set of parameters $\Gamma$. Positively and negaltively charged muon pairs were handled by dividing their likelihood out of the likelihood function. The CERN library routine MINUIT[110][111] was used to search for the minimum of the function. Each time the function was called, by MINUIT, the likelihood of each data event was revaluated wi th the new parameters, and the weights for each Monte Carlo event were recalculated. A full fit of the 387 antiproton continuum events using $10^{5}$ Monte Carlo events took 200 seconds of central processor time on the Fermilab Cyber 175 computer [86].

The parameters obtained from the simultaneous rits of these forms to the unbinned data are given in $T$ able 7 along with the errors and correlation coefficients calculated by MINUIT. The $\cos \theta$ distribution was assumed to behave as

$$
1+\cos ^{2} \theta
$$

for these fits. Table 8 gives the results assuming the $\cos \theta$ distribution behaves as

$$
\text { - } 1+\lambda \cos ^{2} \theta,
$$

and allowing the fitting program to determine the best value for $\lambda$. The resulting value of $\lambda=1.06 \pm 0.28$ for the pions is in good agreement with the value of $\lambda=1$ expected from the Drell-Yan model. 'The agreement obtained for the antiprotons is less satisfactory, but it can be seen that the mass, $x_{F}$, and $p_{T}$ distributions are not sensitive to the value of $\lambda$. Both the antiproton and the pion $\cos \theta$ distributions will be discussed $f$ urther in 


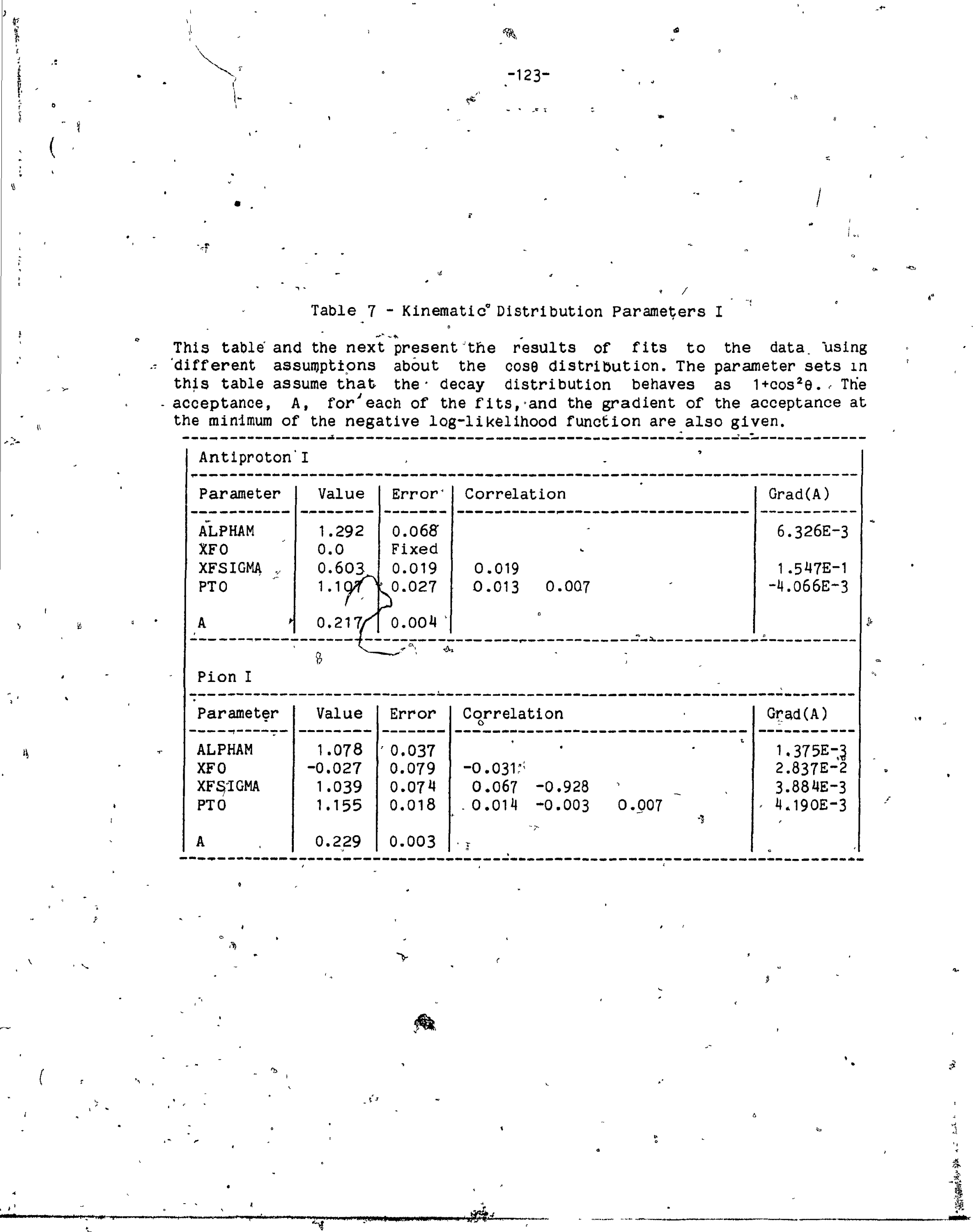




$$
8^{-124-}
$$

Table 8 - Kinematiç Distribution Rarameters II

This table presents. the results of the fits to the data assuming that the $\cos \theta$ distribution behaves as $1+\lambda \cos ^{2} \theta$, and allowing the $f i t t i n g$ programme to $f$ ind the best value for $\lambda$. The acceptance, $A$, for each of the fits, and the gradient of the acceptance at the minimum of the, negative log-likelihood function are also given.

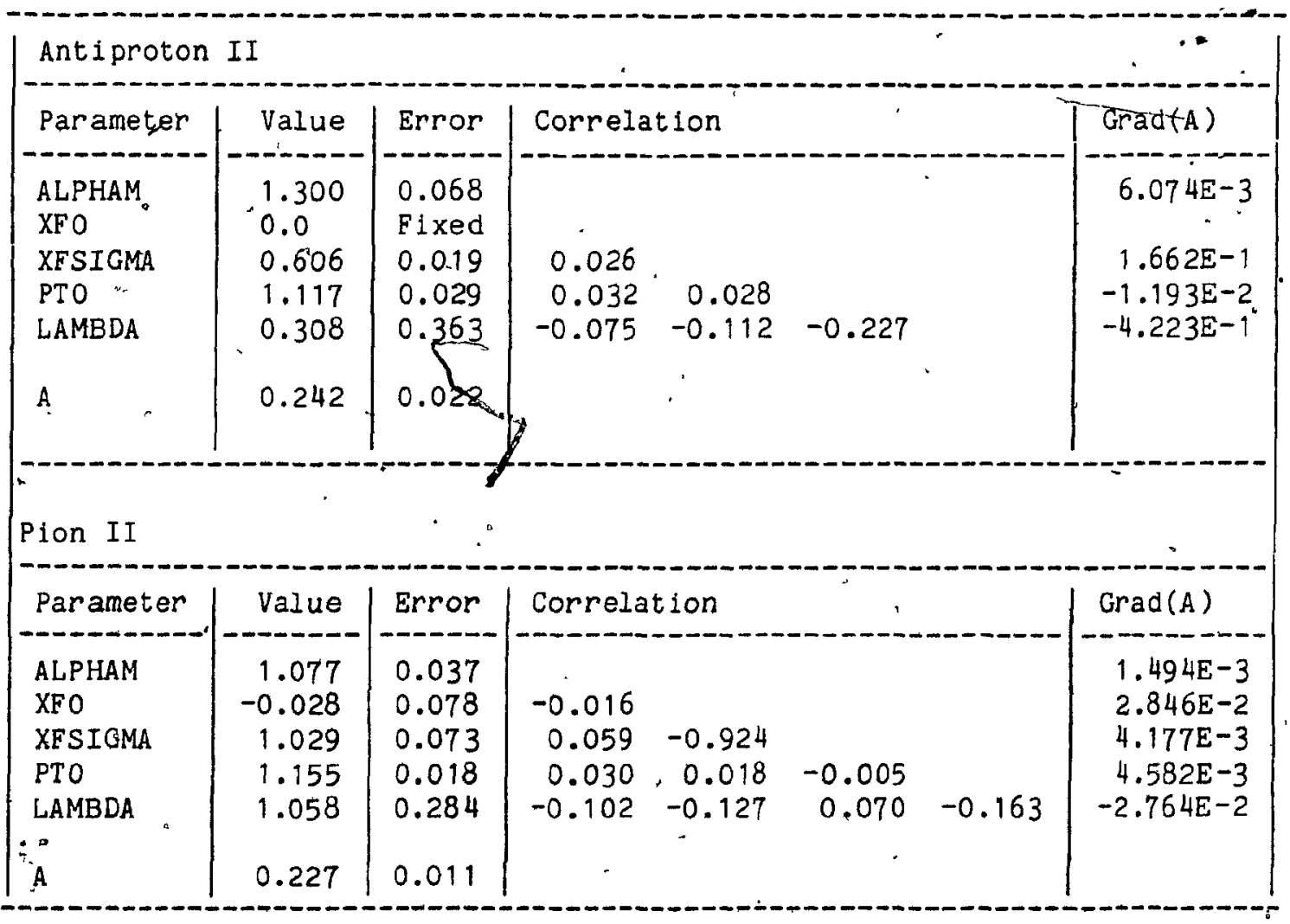


the next chapter."

The mas disteibutions or antiproton and pion

The mass distributions for antiproton and pion produced muon pair events with $x_{F}>0$ are shown in $F$ igure 35 and $F$ igure 36 respectively and compared with the exponentials used to calculate the acceptance. In Figure 37 and Figure 38 the $x_{F}$ distributions for antiproton and pion produced events in the mass range $4, \leq M \leq 9 \mathrm{GeV} / \mathrm{c}^{2}$ are shown with the curves used in the acceptance calculations. The $\mathrm{p}_{\mathrm{T}}, \cos \theta$, and $\phi$ "distributions are presented and discussed in the next chapter.

The kinematic distributions extracted from the data are in good agreement in all cases with the functional forms assumed. For any variable, the distributions extracted from the data should not be sensitive to the parameterization of that variable. The acceptance correction for the mass plot will depend on the parameterizations chosen for the $x_{F}, p_{T}, \cos \theta$, and $\phi$ distributions, but should not depend on the parameterization chosen for the mass distribution. The agreement, between the $x_{F}, p_{T}, \cos \theta$ and $\phi$ dependences extracted and the distributions used to calculate the acceptance leads to confidence in the accuracy of the mass distribution. In the same way the agreement in terms of the other four variables leads to confidence in the $x_{F}$ distribution, and so on for the other parameters.

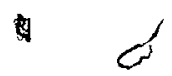

Since the acceptance and the kinematic distributions are "interrelated, it is necessary to determine the uncertainty in the acoeptance due to the uncertainty in the parameters obtained'fram the likelihood fit. Eadie[107] discusses various methods of estimating error intervals for the parameters from a maximum ilkelihood $f 1 t$, but some discussioñ is necessary here. 

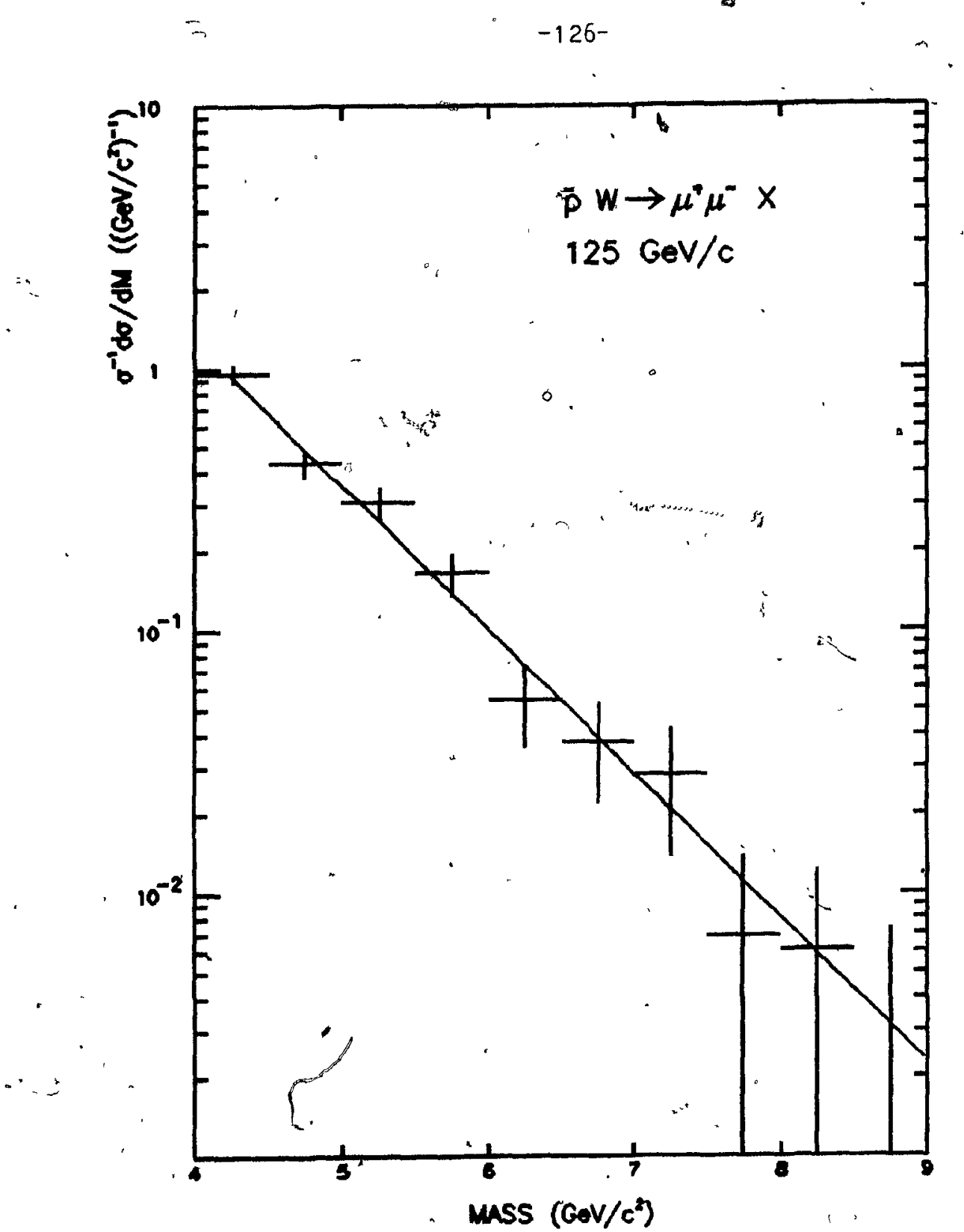

Figure 35 - Antiproton Mass Distribution

The points show the mass distribution of the antiproton produced data. Corrections have been applied for acceptance and reconstruction efficiency. The errors shown are statistical only. The curve shows the exponential $f$ it using the parameter value of $\alpha_{M}=1.30$ from Table 7.$\rangle$. 


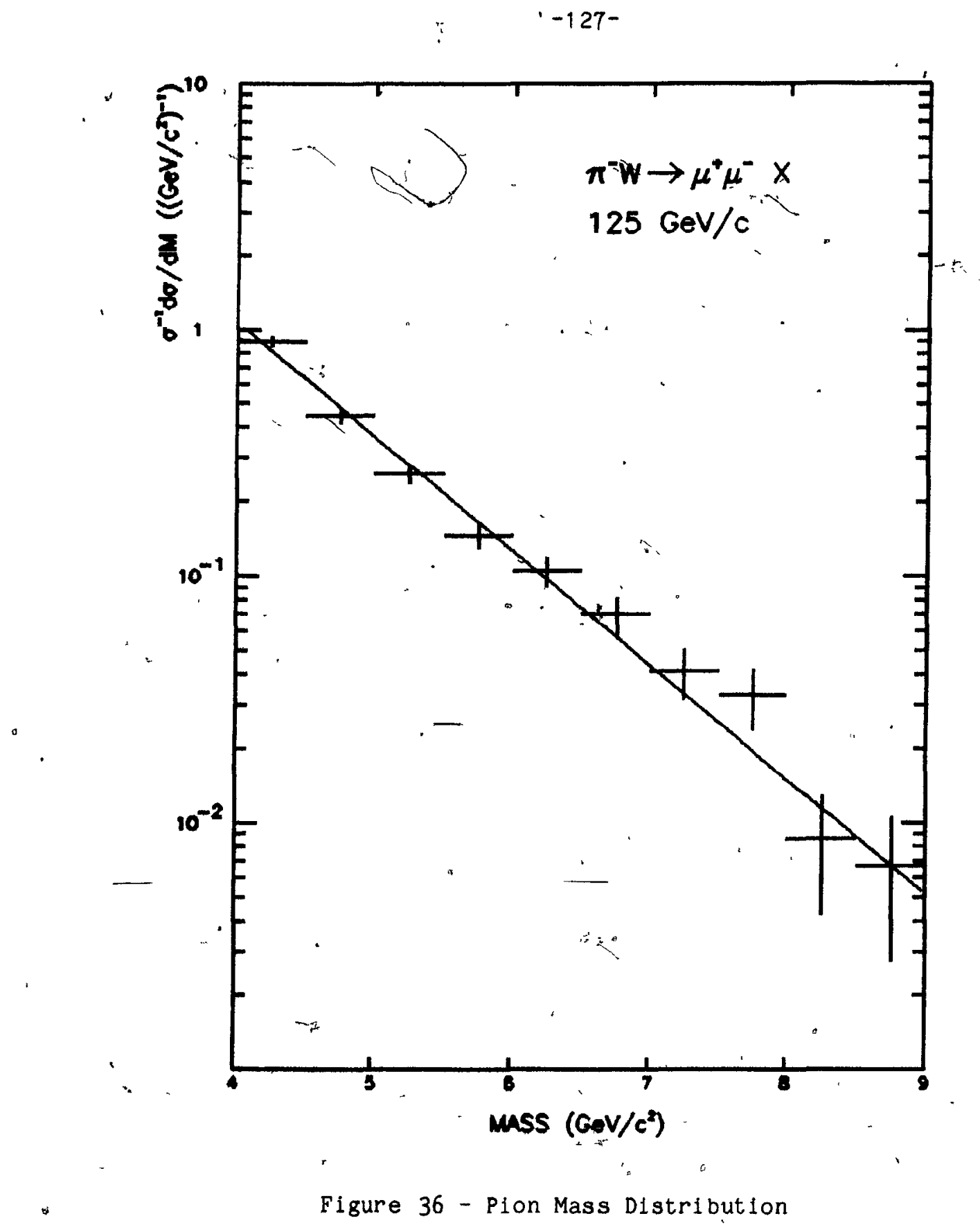

The points show the mass distribution of the pion produced data. Corrections have been applied for acceptance and reconstruction efficiency. The errors shown are statistical only. The curve shows the exponential fit using the parameter value of $\alpha_{M^{\prime}}=1.078$ from Table 7 . 


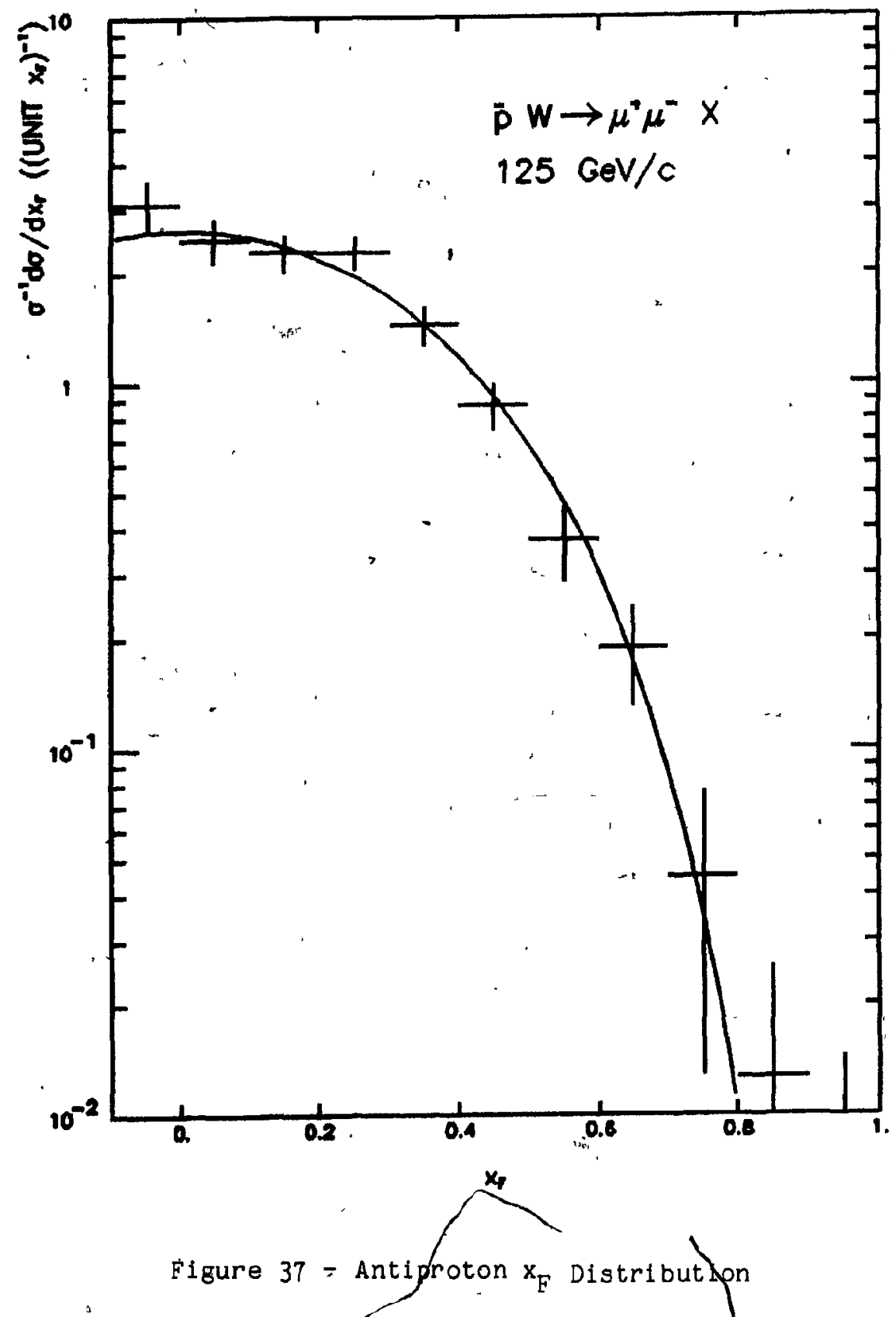

The points show the $x_{F}$ distribution of the antploton produced data. Corrections have been applied for acceptance and recorstruftion ef $f$ iciency. The errors shown are statistical only. The curve shows the Johnson empirical distribution used to parameterize the data. The parameter values obtained from the fit are given in Table 7 . 


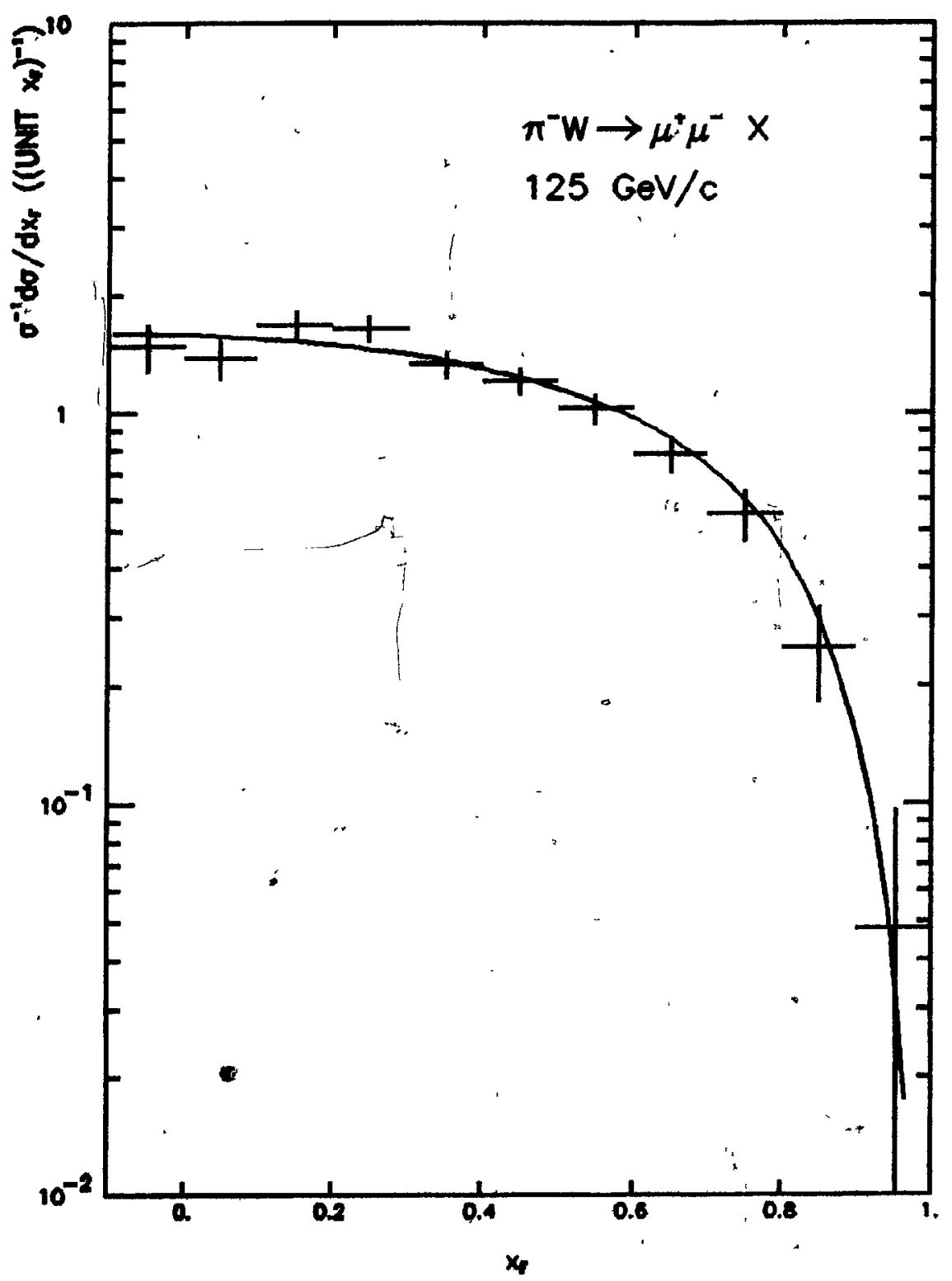

Figure $38^{\circ}-$ Pion $x_{F}$ Distribution

The points show the $x_{F}$ distribution of the pion produced data. Corrections have been applied for acceptance and reconstruction efficiency. The errors shown are statistical only. The curve shows the Johnson empirical distribution used to parameterize the data. The parameter values obtained from the fit are given in Table 7.

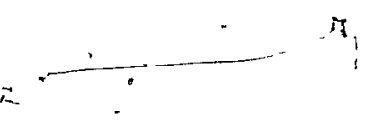


At the minimum, the negative. log-likelinodd function is roughly parabolic. All the first derivatives vanfsh and a Taylor series expansion begins with the quadratic terms. A change in the negative log-likel ihood of $1 / 2$ corresponds to a change in probability of 68.3 percent, in much the same way as a change in $x^{2}$ of 1 does in the usual least squares fit. If we denote the yalue of the negative log-likelinood at the minimum by $L_{0}$, the locus of points where the negative log-likel ihood function has the value

$$
-\ln (L)=-\ln \left(L_{0}\right)+\frac{1}{2} \text {. }
$$

defines a hyper-ellipsoidal surface in the multidimensional parameter space, sometimes called the error ellipsold. This can best be visualised by considering the case of two parameters, $\alpha$ and $\beta$, with a minimum in the negative, negkative log-likelihood function at $\alpha_{0}$ and $\beta_{0}$, as lilustrated in Figure 39 where the the parameter axes and the error ellipsoid are sketched. Error bounds, for a parameter can be evaluated by, projecting the ellipsold on to the parameter axes.

- The same technique can be used to calculate bounds on the error in the acceptance. The gradient of the acceptance at the minimum can be used to define an acceptance axis. The error ellipsold can be projected onto this axis to obtain the values of $\alpha$ and $\beta$ for which the acceptance is an/extremum within the ellipsoid. The acceptance can then be evaluaded at these points to obtain bounds on the acceptance. The usual interdeetation ascribes a 68.3 percent probability that the acceptance lies within these bounds. -

The acceptance of the spectrometer for each of the fits was given in Tables 7 and 8 together with the gradient-of the acceptance at the minimum of the negative log-likelihood function, and the calculated bounds for the acceptance. If the $\cos \theta$ distribution is $1+\cos ^{2} \theta$, the probable error made in 


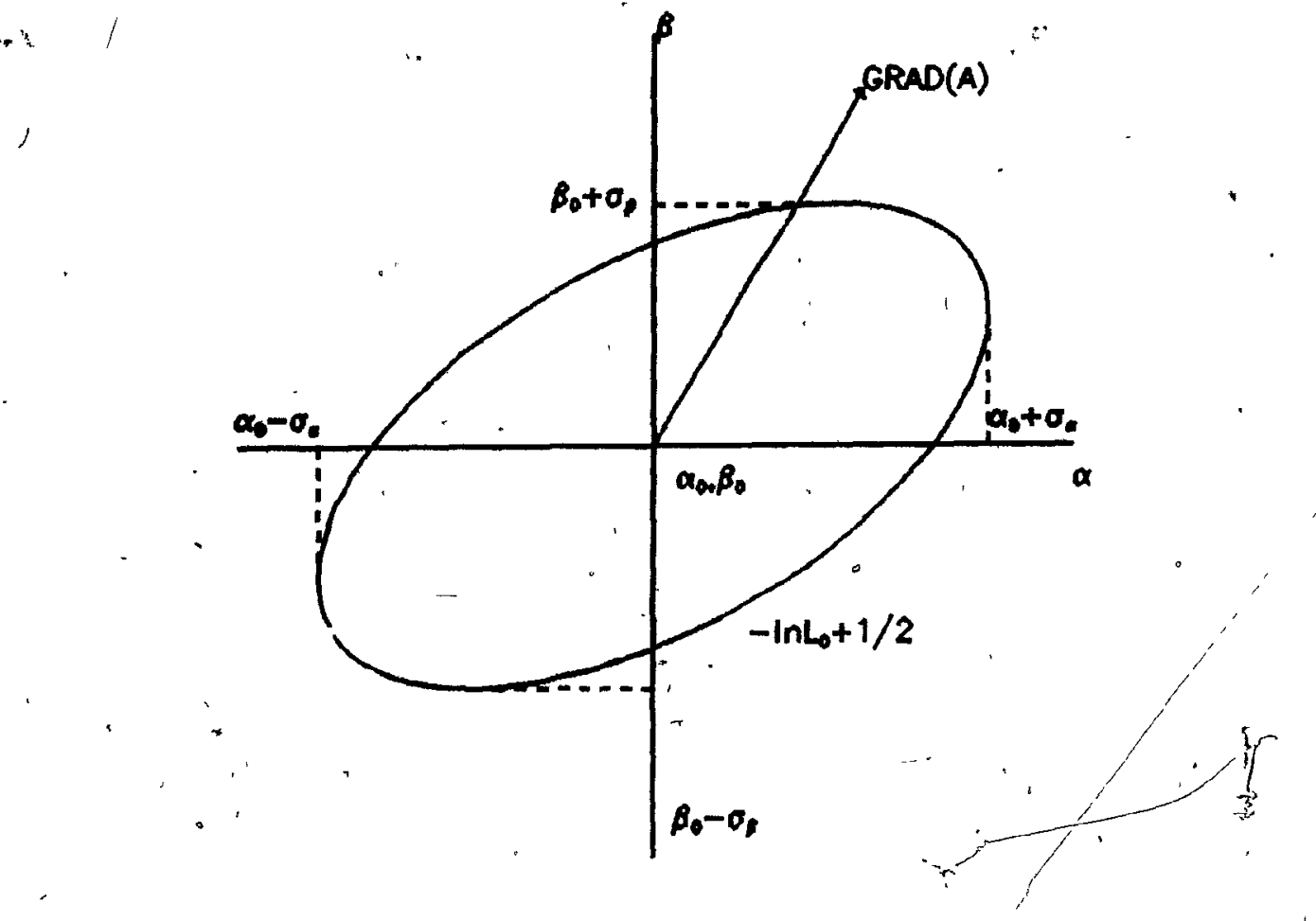

Figure 39 - Error Ellipsoid

The surfaces of constant likelihood oan be used to give error bounds on the parameters by projecting the error ellipsoid onto the parameter axes as discussed in the text. The ellipsoid can also be projected onto the gradient of the acceptance to give bounds for the error in the acceptance calculation as explained in the text. 
calculating the acceptance is 2 percent for the antiproton produced data and 1.2 percient for the pion produced data, based on the parameter sets of Table 7. Allowing the fit to determine the value of $\lambda$, that'is, using the parameter sets of Table 8 , the probable error made in calculating the acceptance is 9.1 percent and 5 percent respectively for the antiproton and the plon produced data.

6.2 Reconstruction, Counter, and Trigger Efficiencies

As discussed in the last chapter, the correction for track finding efficiency was included in the calculation of the acceptance by requiring the Monte Carlo events to be reconstructed by the analysis programmes. The 1 uncertalnty in the total cross section due to uncertainties in the track finding efflciency correction was calculated to be less than 4 percent. The maximum error in any of the differential cross sections, due to uncertaintles in the correction for track finding efficlency, is calculated to be less than 8 percent. The cross section was also corrected for the measured counter and trigger of ficlencies.

When the absorber counter was used in the trigger, a subsample of events which did not require the counter was recorded so that the efficiency of the, counter could be studled. Reconstructed muon tracks - were projected back to the counter and the latch information for these events was examined to see if the counter had been hit in colncidence with the trigger. The overall efficlency was calculated in this way to be

$$
\epsilon\left(\text { ABSR }_{1982}\right)=0.9700 \pm 0.0012 \text {, }
$$

for the 1982 run, and

$$
\varepsilon\left(\text { ABSR }_{1981}\right)=0.969 \pm 0.004 \text {. }
$$


for the 1981 run.

Events from special runs with the respective hodoscope signals removed from the trigger were used to calculate the CPX and CPY efflolenoles. The efflclencies were found to be as follows for the various runs:

$$
\begin{aligned}
& \varepsilon\left(C P X_{1982}\right)=0.9844 \pm 0.0020, \\
& \varepsilon\left(C P Y_{1982}\right)=0.9900 \pm 0.0016, \\
& \varepsilon\left(C P X_{1981}\right)=0.9877 \pm 0.0055, \text { and } \\
& \varepsilon\left(C P Y_{1981}\right)=0.9869 \pm 0.0058 .
\end{aligned}
$$

The efficiencies of the muon counter arrays were studied in a special run using coincidences between only two out of the three planes in the trigger. The efficiencies of the three muon planes were calculated to be

$$
\begin{aligned}
& \varepsilon(\mu 1)=0.9810 \pm 0.0013, \\
& \varepsilon(\mu 2)=0.9980 \pm 0.0004 \text {, and, }
\end{aligned}
$$

$\varepsilon(\mu 3)=0.9970 \pm 0.0005$.

Combining these numbers yields overall trigger efficlencies for the various "runs as follows:

$$
\begin{aligned}
& \left.\varepsilon \text { (TRIG1 }_{1982}\right)=0.905 \pm 0.004 \pm 0.012, \\
& \left.\varepsilon \text { (TRIG2 }_{1982}\right)=0.883 \pm 0.004 \pm 0.013, \\
& \left.\varepsilon \text { (TRIG1 }_{1981}\right)=0.905 \pm 0.011 \pm 0.027 \text {, and } \\
& \varepsilon\left(\text { TRIG2 }_{1981}\right)=0.877 \pm 0.012 \pm 0.031 .
\end{aligned}
$$

The first error quoted for each efflciency. is the result, of adding the individual errors in quadrature. The second error is the result of assuming 


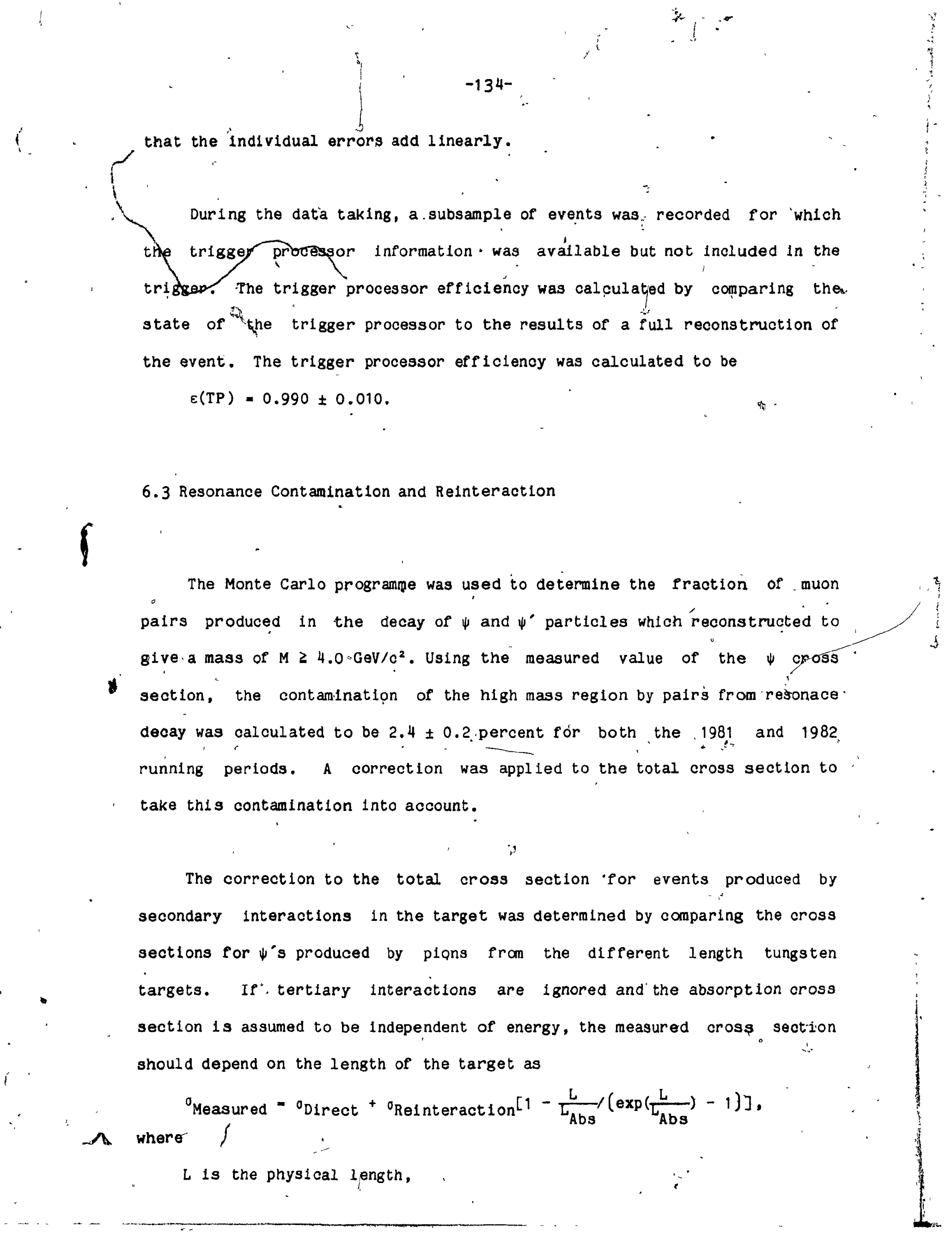


$L_{A b s}$ is the absorption length of the target material,

${ }^{\text {ODirect }}$ is the cross section that would be measured using an infinitesimally thin target, and

OReinteraction is a constant that depends on the details of the reinteraction but is independent of the target, length.

Measured cross sections for different Iength targets can therefore be used to "obtain these parameters. This equation is discussed further in Appendix III.

$\therefore$

The relative production rates for $\psi$ 's from the front half of the 1981 fungsten target, the 1982 thick tungsten target, and the 1982 thin turigsten target were found to be $2.728 \pm 0.082,2.715 \pm 0.057$, and $2.831 \pm 0.042$ respectyvely and are plotted in Figure 40. Fitting these values with the above expersign gives and $0_{\text {Direct }}=2.655 \pm 0.078$,

OPeinteraction $=0.356 \pm 0.202$, so that. the correction for any length of target can be determined. .

The Monte Carlo programme CASIM[112] was used to extrapolate fram pion produced $\psi$ events to the high mass region. CASIM uses the Hagedorn-Ranft thermodynamic model to generate a spectrum of secondary particles. The measured $\tau$ dependence of the Drell-Yan cross section[18] was used to gerierate high mass muon palr events from the spectrum of secondaries. The pairs were propagated through the spectrameter with the simulation program and used to determine the reinteraction rate for the antiproton beam and the high mass region relative to pion produced $\psi^{\prime} s$. The correction factors for the cross section with, varlous target and beam combinations are given in Table 9. 
Table 9 - Reinteraction 'Correction"

These factors have been applied to the various subsets of data to correct for reinteraction. The estimated errors on the corrections are given below the numbers.

\begin{tabular}{|c|c|c|c|c|c|}
\hline & $\mathrm{BE}$ & $\mathrm{CU}$ & W & THICK W & , ${ }_{0}$ THIN,$W$ \\
\hline \multicolumn{6}{|c|}{ PSI REINTERACTION CORRECTION } \\
\hline PBAR & $\begin{array}{l}.95440 E+00 \\
.25233 E-01\end{array}$ & $\begin{array}{l}.95502 E,+00 \\
.24893 E-01\end{array}$ & $\begin{array}{l}.95519 E+00 \\
.24795 E-01\end{array}$ & $\begin{array}{l}.93899 \mathrm{E}+00 \\
.33760 \mathrm{E}-01\end{array}$ & $\begin{array}{l}.97922 E+00 \\
.1,1499 E-01\end{array}$ \\
\hline PION & $\begin{array}{l}.95799 E+00 \\
.23248 E-01\end{array}$ & $\begin{array}{l}.95442 E+00 \\
.25223 E-01\end{array}$ & $\begin{array}{l}.95231 E+00 \\
.26392 E-01\end{array}$ & $\begin{array}{l}.93385 E+00 \\
.36603 E-01\end{array}$ & $\begin{array}{l}.97832 \mathrm{E}+00 \\
: 11996 \mathrm{E}-01\end{array}$ \\
\hline \multicolumn{6}{|c|}{ CONTINUUM REINTERACTION CORRECTION , , } \\
\hline PBAR & $\begin{array}{l}.96580 \mathrm{E}+00 \\
.18925 \mathrm{E}-0^{\circ} 1\end{array}$ & $\begin{array}{l}.96626 \mathrm{E}+00 \\
.18669 \mathrm{E}-01\end{array}$ & $\begin{array}{l}.96640 \mathrm{E}+00 \\
.18596 \mathrm{E}-01\end{array}$ & $\begin{array}{r}95424 E+00 \\
\because .25320 E-01\end{array}$ & $\begin{array}{l}.98442 E+00 \\
.86243 E-02\end{array}$ \\
\hline PION & $\begin{array}{l}.97479 E+00 \\
.13949 E-01\end{array}$ & $\begin{array}{l}.97265 E+00 \\
.15134 E-01\end{array}$ & $\begin{array}{l}.97138 E+00 \\
.15835 E-01\end{array}$ & $\begin{array}{l}.96031 E+00 \\
.21962 E-01\end{array}$ & $\begin{array}{l}. \$ 8699 \mathrm{E}+00 \\
.71974 \mathrm{E}-02\end{array}$ \\
\hline
\end{tabular}


The size of the corrections are less than 5 percent for the high mass antiproton data and less than 4 percent for the pion data? The uncertainties in the corrections lead to a 2 percent uncertainty in the . cross sections.

\section{4. Beam Flux}

The number of beam particles hitting the target was counted directly by the Cerenkor counters and beam hodoscopes. Corrections were made to, the flux totals to take into/account the probability that two beam particles travelling through the beam telescope at the same time, that 1s, in the same RF bucket, would not be retoed by the $28 Y \geq 2$ logic. Since no conf usion could arise if both particles were plons or if both particles were antiprotons, the flux totals were increased to take these cases into account. Because even a small contamination. of pions could blas the antiproton kinematic distributions, all events for whlch both Cerenkov counters had recorded a beam particle were eliminated from the data sample. The flux totals were correspondingly decreased to take this case into account. :

The beam flux totals were taken to be

$$
P_{\text {PBAR }} \text { TOTAL }=(P B A R+2 P B A R \cdot V P \cdot H P-(P I \cdot P B A R))\left(B=A M_{L I V E} / B E A M\right)=
$$

for antiprotons, and

$$
P I_{\text {TOTAL }}=(\mathrm{PI}+2 \mathrm{PI} \cdot \mathrm{VP} \cdot \mathrm{HP}-(\mathrm{PI} \cdot \mathrm{PBAR}))\left(\mathrm{PI} \mathrm{PRESCALED}_{\mathrm{PI}} / \mathrm{BEAM}_{\mathrm{Z} I V \mathrm{~V}^{\prime}} / \mathrm{BEAM}\right)
$$

for pions, where the various terms are definerd as follows:

PBAR is the total number of colncidences between a signal in the antiproton "Cerenkov counter, CS1, and the BEAM signal, Indicating the number of antiprotons which had travelled through the beam telescope; 
PI is the total number of colncidences between a signal in the pion Cerenkov counter, CS2, and the BEAM signal, indicating the number. of plons which had travelled through the beam telescope;

$2 P B A R \cdot V P \cdot H P$ and $2 P I \cdot V P \cdot H P$ are the estimated number of pairs of antiprotons (2PBAR) or pions (2PI) that had travelled through the , beam telescope at the same time but were not detected by the $2 B Y \geqq 2$ veto or rejected by the HALO veto logic; the probability that of beam particle would survive the $2 B Y \geq 2$ veto (VP) or the HALO veto (HP) are discussed below;

(PI-PBAR) is the total number of coincidences between the PI and PBAR signals indicating the "number of times that both a pion and antiproton had traversed the beam telescope at the same time;

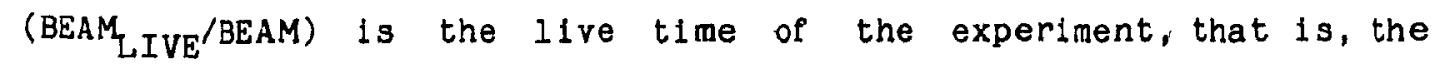
'fraction of BEAM signals counted while the experiment was not busy reading, out a previous event; the live time was calculâted on a spill by splil basis and was typically between 80 and 95 percent depending on the running conditions; and

(PI PRESCALED/PI) is the fraction of pions that passed the pion prescale logic; this fpaction was typically between $1 / 4$ and 1 depending on the setting of the pion prescaleunit. This unit was set at the beginning of each run to maintain a reasonable balanoe between the overall trigger rate and the experimental dead time and depended on the beam spill structure and intensity.

The probability (or formation efficiency, FE) that two beam particles traversing the beam telescope at the same time would give a $2 B Y \geq 2$ signal was calculated by supérimposing beam tracks from the data tapes and determining what fraction of the tracks passed through the same counters in at least two 
of the beam stations. The formation efflolency was calculated to be

n $\quad \mathrm{FE}=0.851$

for the 2 out of 3 beam station veto condition. The probability that two beam particles will not be vetoed is then

$V P=(1-F E)$.

The number of times that two beam particles travelled through the beam telescope can be calculated from the number of $2 B Y \geq 2$ signals counted as $2 B E A M=(2 B Y \geq 2) / F E$.

Poisson statistics were used to determine the number of times two antiprotons (2PBAR) or two plons (2PI) had travelled through the tel escope from the number of times two beam particles (2BEAM) had travelled through the beam telescope and from the ratio of the number of antiprotons to plons in the beam.

The probability thatt. a beam particle would not be vetoed by a HALO signal was calculated as

$H P=B E A M /[3 B Y-(2 B Y \geq 2)]$.

where $3 B Y$ was the total number of colncidence signals from the throe bean stations. HP was typically about 0.96 depending on the running conditions.

The corrections were applied to the beam flux totals on a spill by spill basis. The average corrections to the totals were 0.6 percent" for antiprotons and 4 percent for pions. Depending on the intensity, spili structure, and formation efficiency, the correction to the pion total reached 10. percent for some runs. The final flux totals for the varlous target configurations are given in Table 10. The uncertainty in the cross sections due to uncertainties in the corrections to the beam totals is less than 1.5 percent. $\therefore$ 
Table 10 - Incident Bean Flux by Target Configuration

This table summarizes the numbers of beam particles hitting the various targets. The number of beam particles of each type hitting, the target was counted directly by the Cerenkov counters and beam hodoscopes. All corrections discussed in the text have been applied. The errors in the flux totals are calculated to be less than 1.5 percent for both antiprotons and pions.

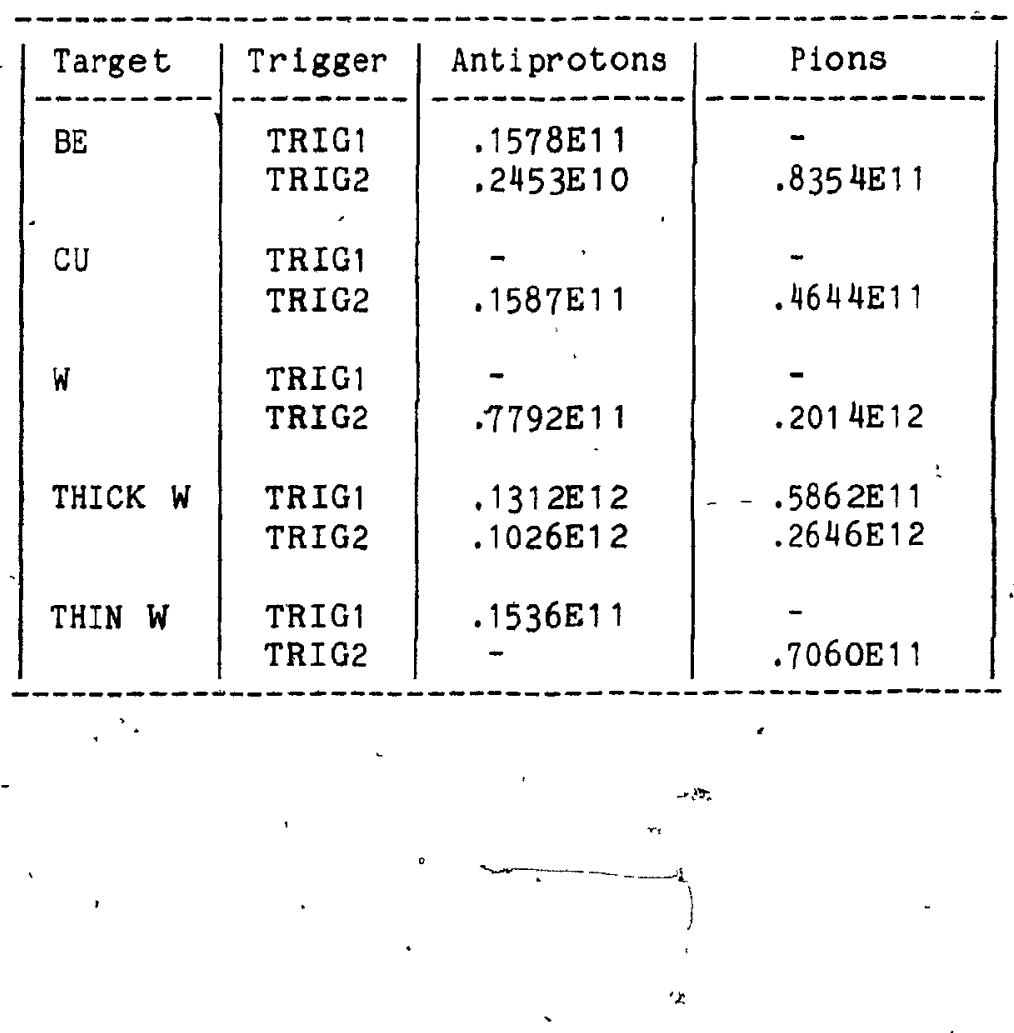




\section{$-142-$}

\subsection{Cross Sections}

Once the parameters were obtained prom the maximum likelihood $f i t$, the data events, were binned in terms of the kinematic variables. Like charge

* muon pairs were subtracted on a bin by bin basis.: The acceptance for each." bin was calculated using the technique discussed above, and the data points corrected for acceptance. The differentlal cross section for each bin in the kinematic variable $x$ was then calculated using the formula

$\frac{d o}{d x}=\left[A \cdot R \cdot N_{\text {Events }}\right] /\left[\Delta x \cdot N_{0} \cdot \rho \cdot L_{E f f} \cdot \xi \cdot E \cdot N_{\text {Beam }}\right]$ where

$x$ is one of the kinematic variables, $M, x_{F}, p_{T}, \cos \theta$ or $\phi$, $\frac{\mathrm{do}}{\mathrm{dx}}$ is the differential cross section in $\mathrm{cm}^{2} /$ nucleon assuming an $\mathrm{A}$ dependence of $A^{1}, \cdots$

$\Delta x$ is the width of the bin,

A is the atomic number of the nuclear target,

No is Avagadro's number,

$\rho$ is the density of the target in $\mathrm{gm} / \mathrm{cm}^{3}$,

$L_{\text {Eff }}$ is the effective length of the target,.

$R$ is the correction for reinteraction and resonance contamination,

$\xi$ is the acceptance for the bin,

$E$ is correction for counter and trigger efflciency,

$N_{\text {Events }}$ is the number of data events in the bin, and

$\mathrm{N}_{\text {Beam }}$ is the number of Team particles hitting the target.

Each of the nucleár targets used was welghed and measured. The length, density, absorption length and effectlve length of each target are given, in Table 2. The absorption length for each target materlal was interpolated 
Prom absorption cross section data for tungsten, copper and beryllium measured with antiprotons and plons[101] at beam energies of 10 and $200 \mathrm{GeV}$. The effective length can be expressed in terms of the physical length of the target and the absorption length as

where

$$
L_{E f f}=L_{A b s}\left[1-\exp \left(-\frac{L}{L_{A b s}}\right)\right] \text {, }
$$

$\mathrm{L}_{\mathrm{Abs}}={ }_{\mathrm{Abs}} \mathrm{PN}_{\mathrm{O}} \mathrm{A}$ is the absorption length of the target material in centimeters,

$L$ is the physical length of the target in centimeters,

${ }^{0}$ Abs is the absorption cross section, and

$\rho, N_{0}$, and $A$ are the target density $\ln \mathrm{gm} / \overline{\mathrm{cm}}^{3}, A$ vagadro's number, and the atomic number of the target material respectively.

Data from targets of different lengths was combined by taking a welghted average of the effective lengths, using the beam flux as the wefghting factor. Uncertalnties in the absorption cross section lead to a 1.7 percent uncertainty in the effective length, land thus in the final cross sections.

- The final cross sections are presented in the next chapter along with comparisons to the Drell-Yan model and leading order QCD calculations. The statistical uncertainty in the total cross section for antiproton produced muon pairs with masses between 4.0 and $9.0 \mathrm{GeV} / \mathrm{c}^{2}$ and $x_{F} \geq 0$ is 5 percent, based on the ' 387 events collected using the tungsten' targets. The statistical uncertainty in the cross section for pion produced pairs is 3 percent based on a sample of 1101 events.

$$
\text { 花 }
$$

Systematic uncertainties in the acceptance, relnteraction correction, counter efficlencies, and beam totals have been discussed in previous sections of this chapter. These errors and their contributions to the 
Table 11 - Systematic Errors

This table gives a summary of the contributions to the systematic error in the measured cross section for antiproton produced muon pairs. Each of the individual component errors, has been discussed in the text. If the components are uncorrelated and the errors add in quadrature, the overall systematic. error is 5 percent. If and the errors add linearly, the overall systematic error is 12 percent.

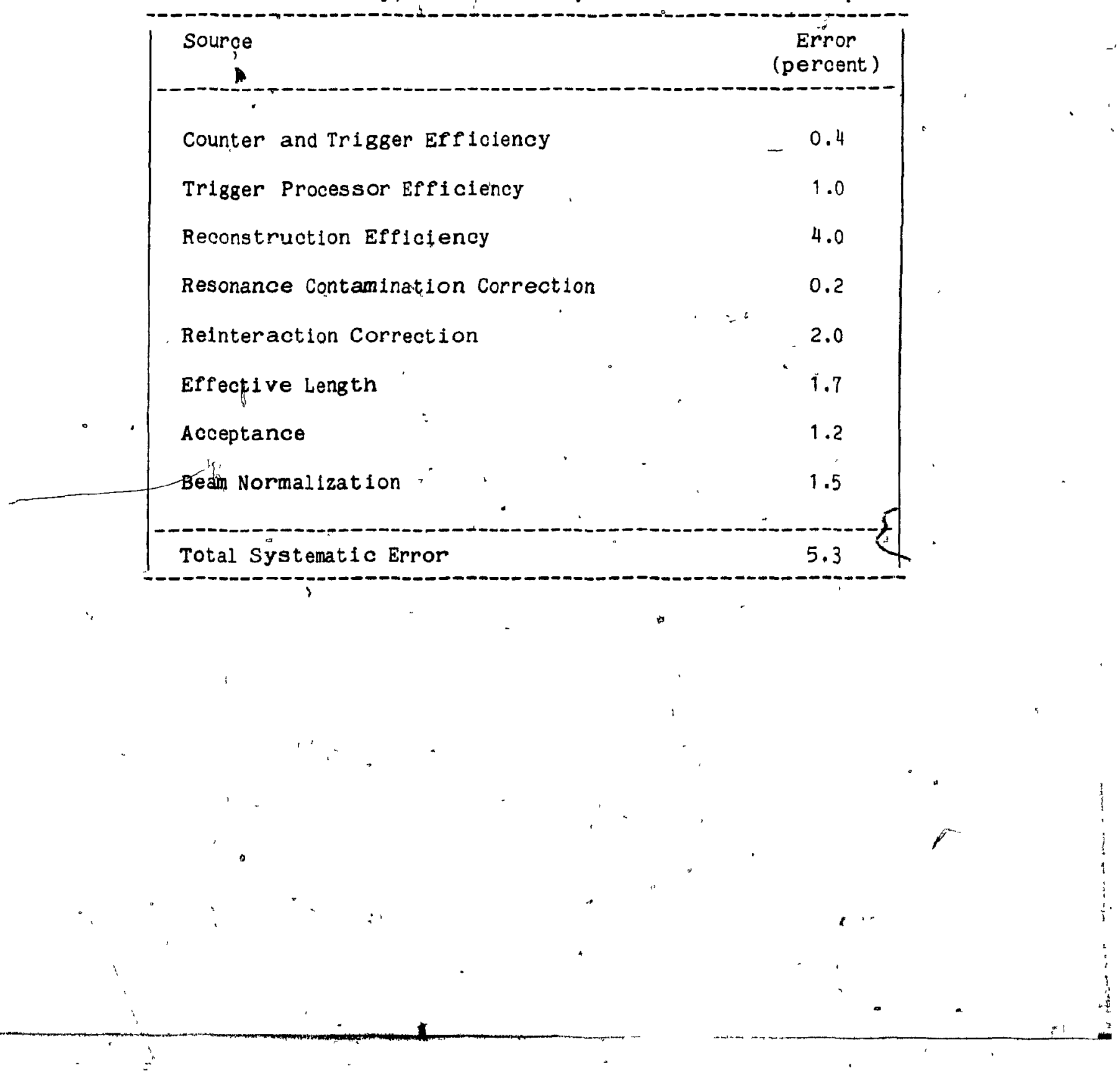


$-145-$

uncertainty in the final cross sections are summarized in Table 11 . The overall systematic error in the total cross section is 5 percent if the Individual components of the error are assumed to be uncorrelated. Alternatively, assuming that the errors are completely correlated gives a.bound of 12 percent. These numbers, of course, depend on the assumptions made in calculating them. If the $\cos \theta$ distribution $1 \mathrm{~s}$ not $1+\cos ^{2} \theta$, and $\lambda$ must be determined from the pits, the systematic error is dominated by the resulting uncertainty in the acceptance that the uncertainty in $\lambda$.. introduces, and the uncorrelated systematic errors must be Increased to 8 and 11 percent respectively for the antiproton and the pion produced data. 
CHAPTER 7

Results

Previous chapters have outlined the reconstruction. of the kinematic variables of the muon, pairs and the methods used to extract the cross sections. This chapter summarlzes the results. The total cross sections for $4 \leq M \leq 9 \mathrm{GeV} / \mathrm{C}^{2}$ are compared to the Drell-Yan model and a leading order QCD calculation. The kinematic distributions are presented and compared to other experiments and the Drell-Yan model. - In the final section the Dreil-Yan model is inverted to extract the valence structure functions of the antiproton and the pion from the data.

\subsection{Total Cross Sections}

The total oross section per nucleon for continum muon.palr production with $4 \leq M \leq 9 \mathrm{GeV} / \mathrm{c}^{2}$ and $x_{F} \geq 0$ is

$$
\sigma_{\bar{p}}=0.104 \pm 0.005 \pm 0.005 \mathrm{nb}
$$




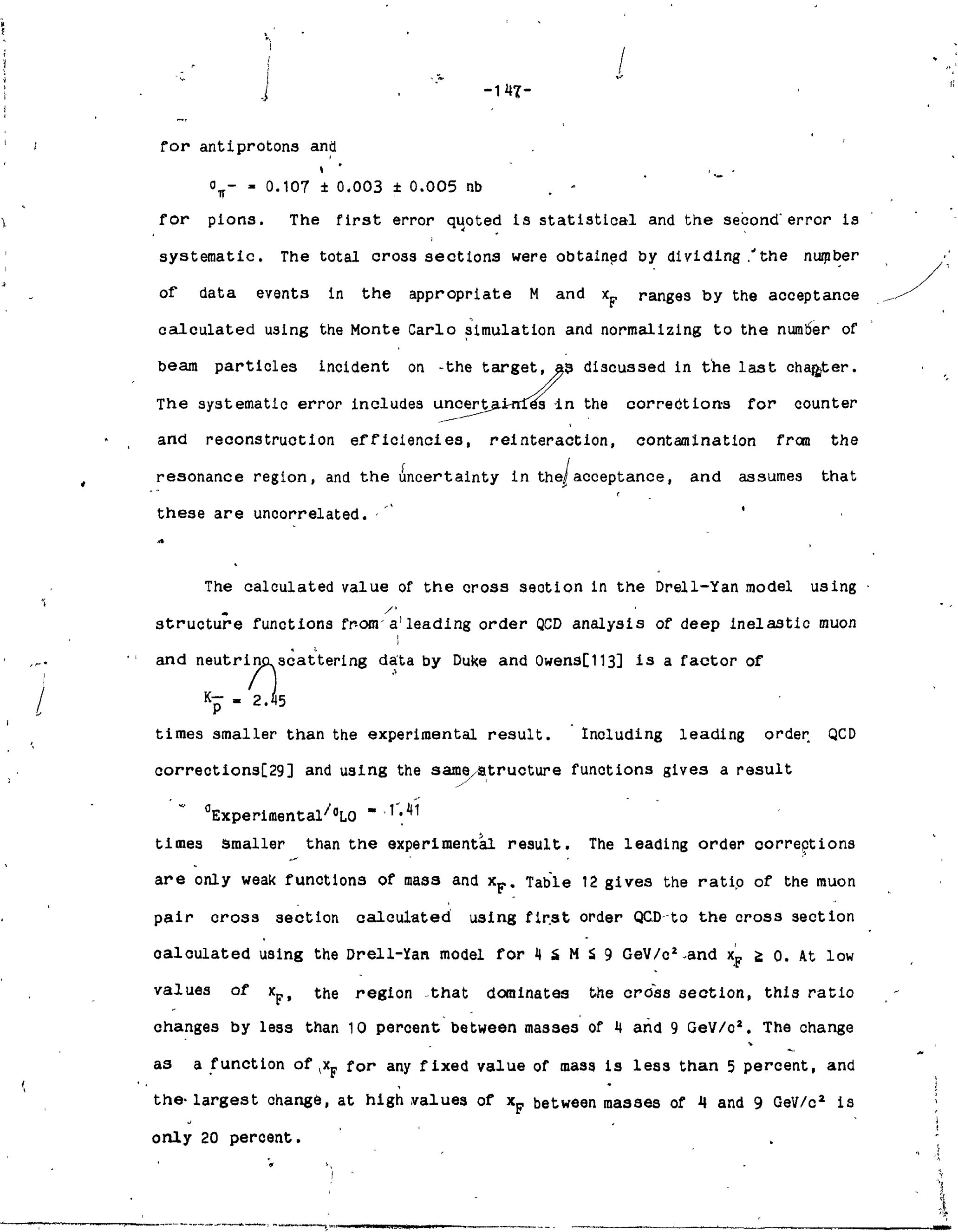




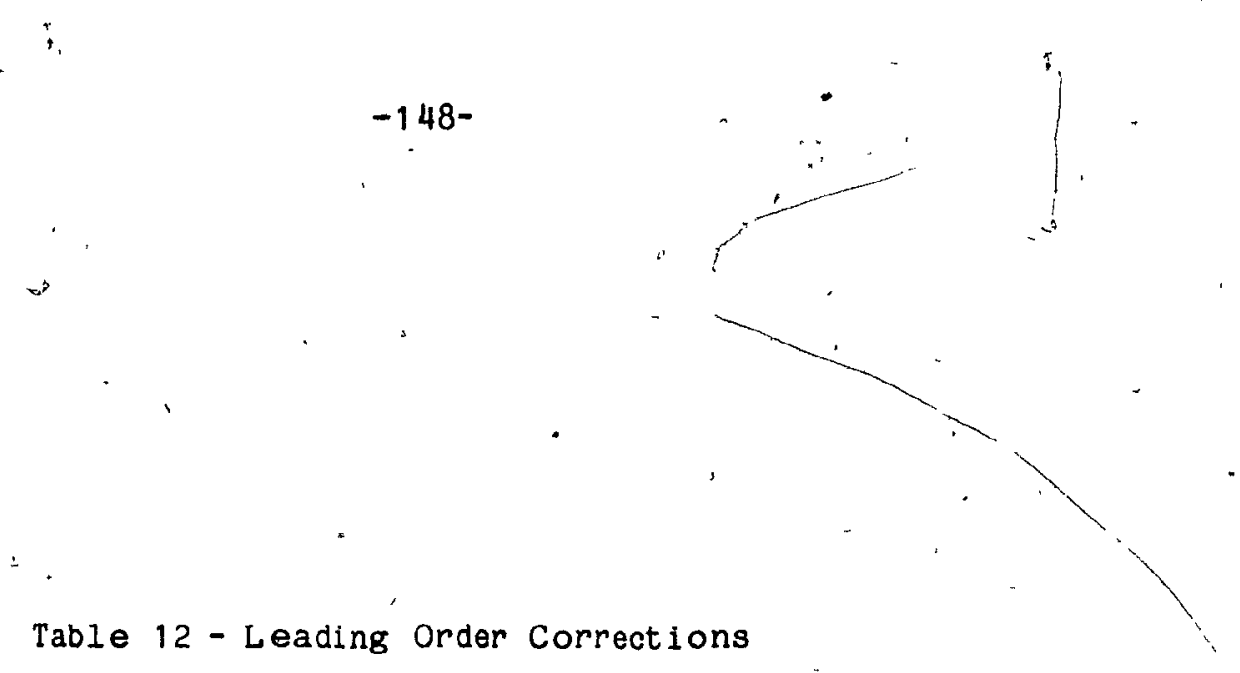

This table presents the ratio of the cross section for muon pair production in antiproton-tungsten collisions calcuated using first order QCD to the cross" section calculated using the parton model as a function of mass and $x_{F}$. The table was calculated using a programme written by $R$.Wagner using equations from Appendix $D$ of Reference 29, and Set 1 structure functions from Reference 113, with a $\Lambda$ of $200 \mathrm{MeV} / \mathrm{c}^{2}$. The programme-used a 6 point Gausgian integration routine[114] from the CERN Program Library to calculate the integrals numerically. Doubling the number of integration steps changed the $K$ factor by less than 0.2 percent at any value of mass and $x_{F}$ in this table.

\begin{tabular}{|c|c|c|c|c|c|c|c|c|c|c|}
\hline$M^{X F}$ & 0.0 & 0.1 & 0.2 & 0.3 & 0.4 & 0.5 & 0.6 & 0.7 & 0.8 & 0.9 \\
\hline $\begin{array}{l}4.0 \\
4.5 \\
5.0 \\
5.5 \\
6.0 \\
6.5 \\
7.0 \\
7.5 \\
8.0 \\
8.5 \\
9.0\end{array}$ & $\begin{array}{l}1.725 \\
1.734 \\
1.744 \\
1.757 \\
1.771 \\
1.787 \\
1.805 \\
1.825 \\
1.847 \\
1.872 \\
1.899\end{array}$ & $\begin{array}{l}1.727 \\
1.735 \\
1.746 \\
1.759 \\
1.773 \\
1.789 \\
1.807 \\
1.827 \\
1.849 \\
1.874 \\
1.901\end{array}$ & $\begin{array}{l}1.727 \\
1.736 \\
1.747 \\
1.760 \\
1.774 \\
1.791 \\
1.809 \\
1.829 \\
1.851 \\
1.876 \\
1.803\end{array}$ & $\begin{array}{l}1.726 \\
1.735 \\
1.747 \\
1.760 \\
1.775 \\
1.792 \\
1.811 \\
1.831 \\
1.854 \\
1.879 \\
1.907\end{array}$ & $\begin{array}{l}1.722 \\
1.733 \\
1.746 \\
1.760 \\
1.776 \\
1.794 \\
1.813 \\
1.834 \\
1.858 \\
1.884 \\
1.912\end{array}$ & $\begin{array}{l}1.716 \\
1.729 \\
1.743 \\
1.759 \\
1.777 \\
1.796 \\
1.816 \\
1.839 \\
1.863 \\
1.890 \\
1.920\end{array}$ & $\begin{array}{l}1.707 \\
1.722 \\
1.740 \\
1.758 \\
1.777 \\
1.798 \\
1.820 \\
1.844 \\
1.870 \\
1.899 \\
1.930\end{array}$ & $\begin{array}{l}1.695 \\
1.714 \\
1.735 \\
1.756 \\
1.778 \\
1.802 \\
1.826 \\
1.852 \\
1.881 \\
1.911 \\
1.945\end{array}$ & $\begin{array}{l}1.679 \\
1.703 \\
1.728 \\
1.754 \\
1.781 \\
1.808 \\
1.836 \\
1.866 \\
1.897 \\
1.931 \\
.1 .968\end{array}$ & $\begin{array}{l}1.654 \\
1.687 \\
1.720 \\
1.754 \\
1.788 \\
1.822 \\
1.857 \\
1.893 \\
1.930 \\
1.970 \\
2.013\end{array}$ \\
\hline
\end{tabular}


Systematic uncertainties in, the antiproton $K$ factor arise from both the theoretical calculation and the experimental measurement. The experimentally measured cross section has an associated statistioal uncertainty of 5 percent and a systematic uncertainty of the same magnitude due to errors in the acceptance, counter efficiencies and beam normali zation.

It is difficult to make a reliable estimate of the systematic errors associated with the calculated cross section. A ten percent uncertainty in the normalization of the structure functions will lead to a 20 percent uncertainty in the predicted Drell-Yan cross section. The normalization of the deep inelastic structure function, $F_{2}$, seems to vary' from experiment to experiment by, up to 20 percent. Duke and Owens normalized their structure function fits to EMC muon-hydrogen data[115]. Based on their discussions[113] and comparisons by experimental groups[116][117], the normalizations of CDHS neutrino-iron[118], EMC muon-deuterium[119], EMC muon-iron[120], BFP muon-1ron[116], CCFRR neutrino-iron[117] and SLAC electron-hydrogen and electron-deuterium[121] scattering data. with respect to EMC muon-hydrogen data are $1.1,7.05,1.03, " 0.98,0.94$, and 0.92 respectively, so that there is a substantial uncertainty in the calculated cross section from this source,alone.

$\because \therefore$ Additional uncertainties arise from other sources as well. Some of the difficulties associated with the extraction of structure functions from the data are discussed by Devoto[122] and by Barker, Martin, and Shaw[123]. In both moment analyses and numerical integrations of the Altarelli-parisi equations, scaling violations depend strongly on the behaviour of the structure. functions in the high $x$ region. This is a region which is 
difficult to probe experimentally, so that the results of the fits can be sensitive to the funçtional form as sumed:

Problems also arise in the extraction of the non-valence terms which are coupled ito an unknown gluon distribution[122][123]. The value of the QCD mass scale parameter, $\Lambda$, obtalned from the fits depends strongly on the assumptions made about the gluon distribution[113]. Duke and Owens[113] obtained a value for $\Lambda$ of 0.2 in the fits discussed above using a relatively soft gluon distribution. Assuming a harder gluon distribution changed the value obtained for $\Lambda^{-1}$ to 0.4 . With structure functions from the fit whloh assumed $a$ hărd gluon distribution, the Drell-Yan contribution to the muon palr cross section.changed by only one percent, and only very small changes in the mass and $x_{F}$ dependence of the cross sections resulted. First order correction terms, however, are directly proportional to

$$
\alpha_{3}=12 \pi /\left[25 \ln \left(Q^{2} / \Lambda^{2}\right)\right]
$$

so that, while the dependence of the correction terms on mass and $x_{F}$ does not change, the correction terms are a factor of

$$
\ln \left(25 / 0.2^{2}\right) / \ln \left(25 / 0.4^{2}\right)=1.27
$$

hlgher at $Q^{2}=25 \mathrm{GeV}^{2} / \mathrm{C}^{4}$ if the, larger value of $\Lambda$ is used.

\subsection{Kinematio Distributions}

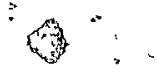

The antiproton produced mass distribution is compared to the predictions of the Drell-Yan model in Figure 41. The component contributions to the mass distribution are shown separately. The Drell-Yan predictions were calculated using the deep inelastic structure functions[113] discussed previously, and weremultiplied by a factor of $=\quad \mathrm{k}-2.45$ 


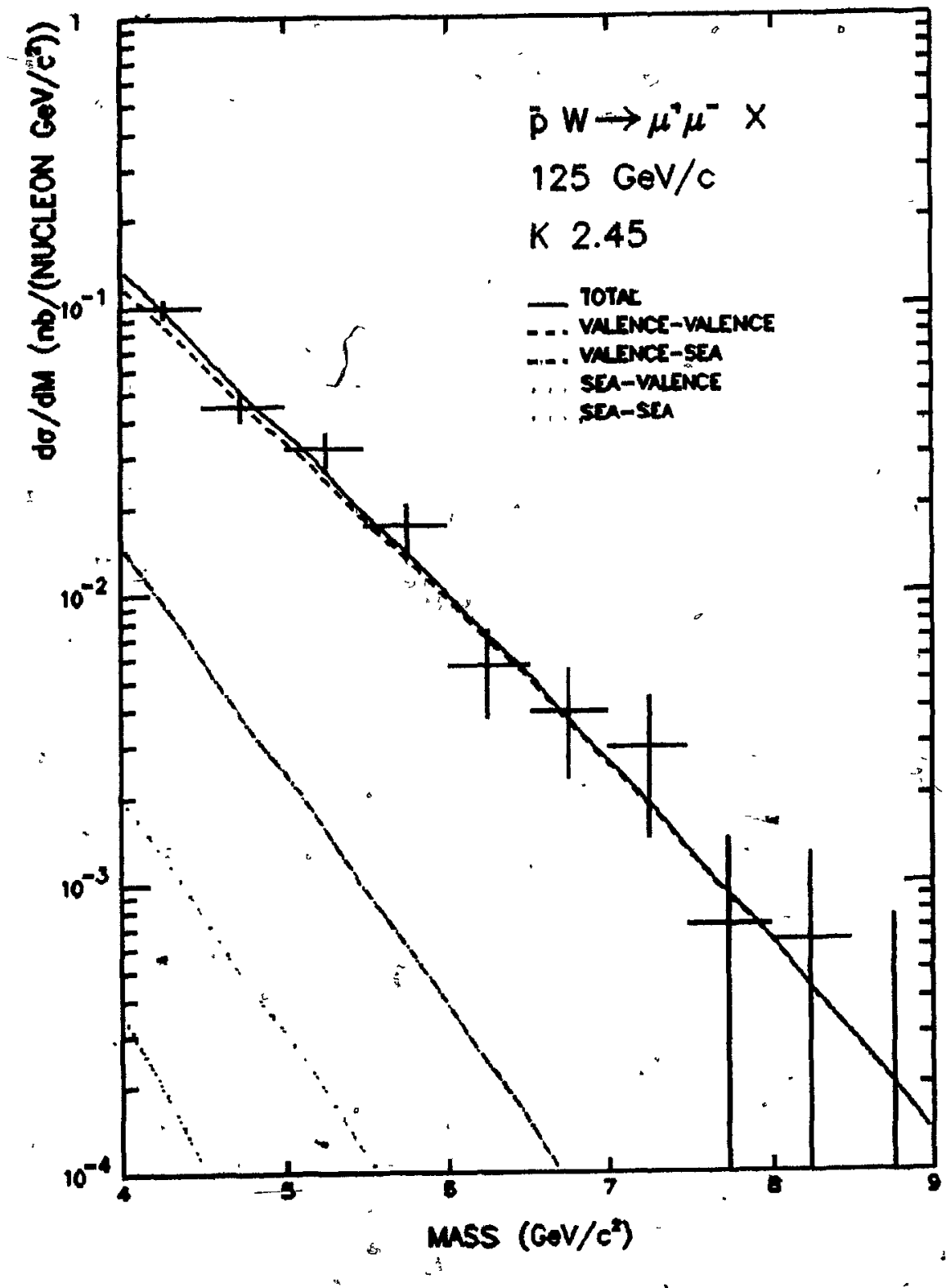

Figure 41 - Ant1proton Mass Distribution Compared To Drell-Yan-Prediction

The points show the mass distribution of the antiproton produced data. The solid line shows the shape of the cross section predicted by the Drell-Yan model using structure functions from measurments of deep-inelastic "scatteri"ng for both the antiproton and the nucleon. The curve, has been multiplied by a factor of 2.45 to reproduce the measured total cross section for $4.0 \leq M \leq 9.0 \mathrm{GeV} / \mathrm{c}^{2}$ with $x_{F} \geq 0$. The other curves show the components of the predicted cross section as indicated. Note that the anninilation of valence antiquarks from the antiproton with valence quarks from the nucleon accounts for more that 90 percent of the total. 
to reproduce the measured total cross section. The antiproton cross section is dominated over the entire mass region by the valence-valence terms,

The pion produced mass spectrum is compared to the Drell-Yan model in Figure 42. For thls piot, the beam structure function was extracted from our data as discussed later in this chapter, while the nucleon structure function was -taken from the deep inelastic scattering results[113]. The curves were multiplled by a factor of

$$
K_{\pi^{-}}=2.39
$$

so as to reproduce the measured choss section. Again the component distributions are shown separately.

In Figure 43 and Figure 44 the $x_{F}$ distributions are shown together with the Dreli-Yan predictions. Again the calculations have been mult tplied by the empirical $K$ factors necessary to reprquce the experimentally measured cross sections. As with the mass distributions the separation of the predictions into the component curves shows the extent to which both the antiproton and pion processes are dominated by valence-valence anninilation. Counting rule arguments[124][125] suggest that the $x_{F}$ distribution for.pions . should be platter than that for antiproton events, and this is confirmed by the data.

The shapes of the antiproton mass and $x_{F}$ distributions are sensitive tests of the Drell-Yan model. Leading order QCD corrections have little effect other than changing the normalization. The Drell-Yan model describes both of these distributions very well.

The $\mathrm{P}_{\mathrm{T}}$ distributions for antiproton and plon produced events with $r$ 


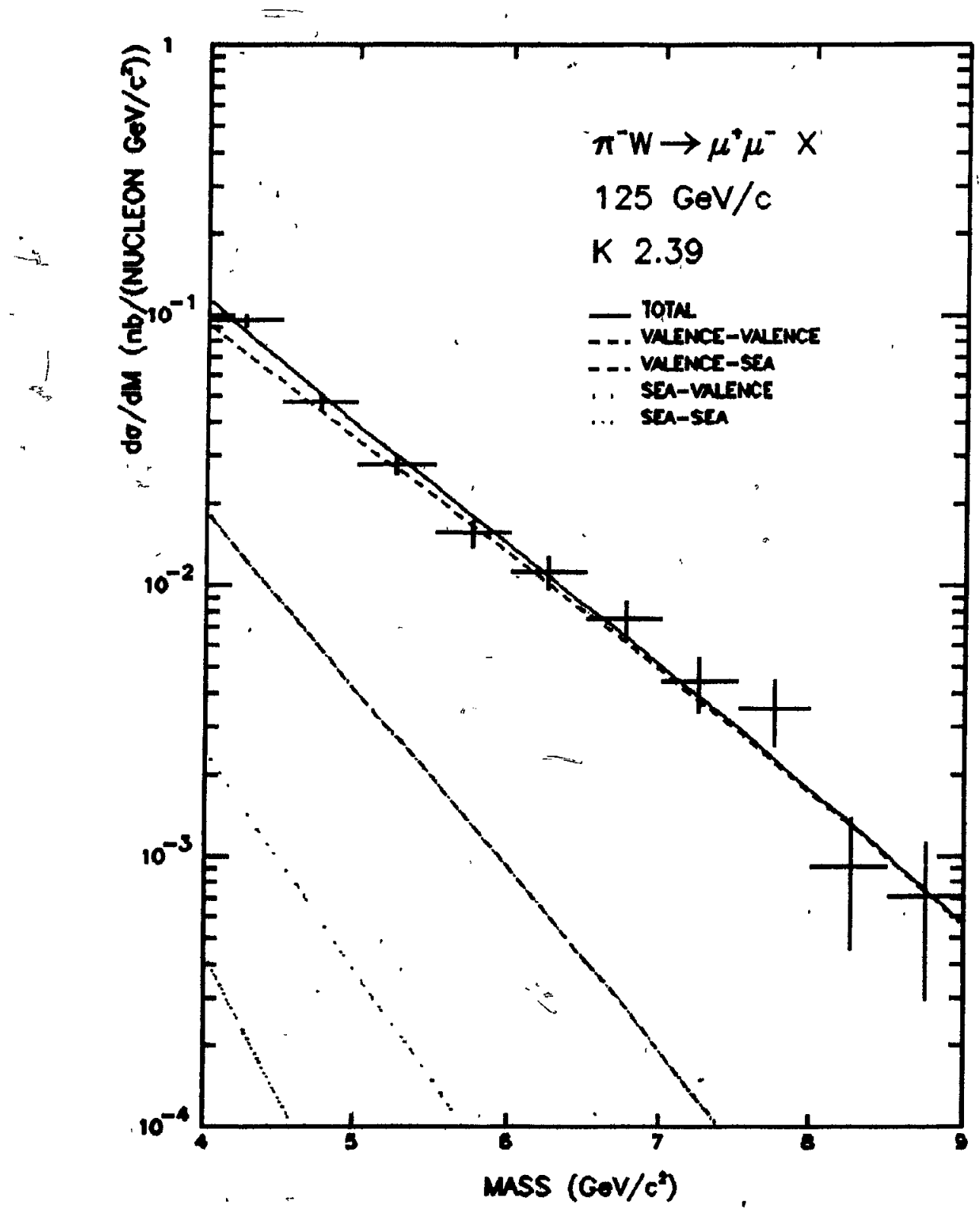

$=$ Figure 42 - Pion Mass Spectrum Compared To Drell-Yan Prediction

The points show the mass distribution of the pion produced data. The solid line shows the shape of the cross section predicted by the Drell-Yan model using structure functions from measurments of deep-inelastic scattering for the nucleon, and our fits for the pion structure function. The curve has been multiplied by a factor of 2.39 to reproduce the measured total cross section for $4.0 \leq M \leq 9.0 \mathrm{GeV} / \mathrm{c}^{2}$ with $x_{F} \geq 0$. The other curves show the components of the predicted cross section as indicated. 


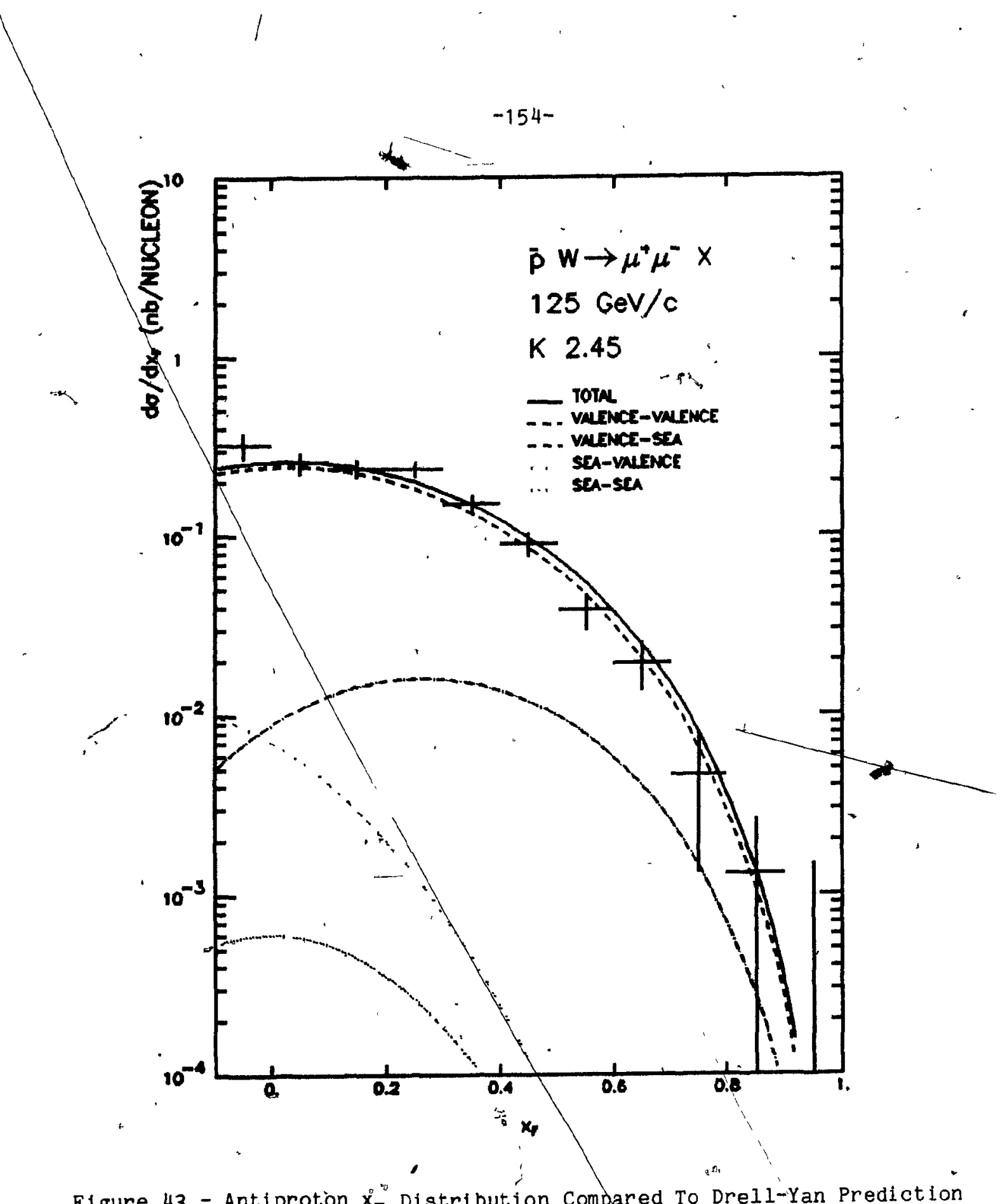

Figure 43 - Antiprotion $x_{F}$ Distribution Compared To Drel1-Yan Prediction

The points sho the $k_{F}$ distribution of the antiproton produced data. The solid line shows the shape of the cross section predicted by the Drell-Yan model using structure functions from measurments of deep-inelastic scattering for both the aritiproton and the nucledn. The curve has been multiplied by a factor of 2.45 to reproduce the measured total 'onoss section for $4.0 \leq M \leq 9.0 \mathrm{GeV} / \mathrm{c}^{2}$ with $x_{F} \geq 0$. The other curves show the components of the predicted cross section as indicated. 


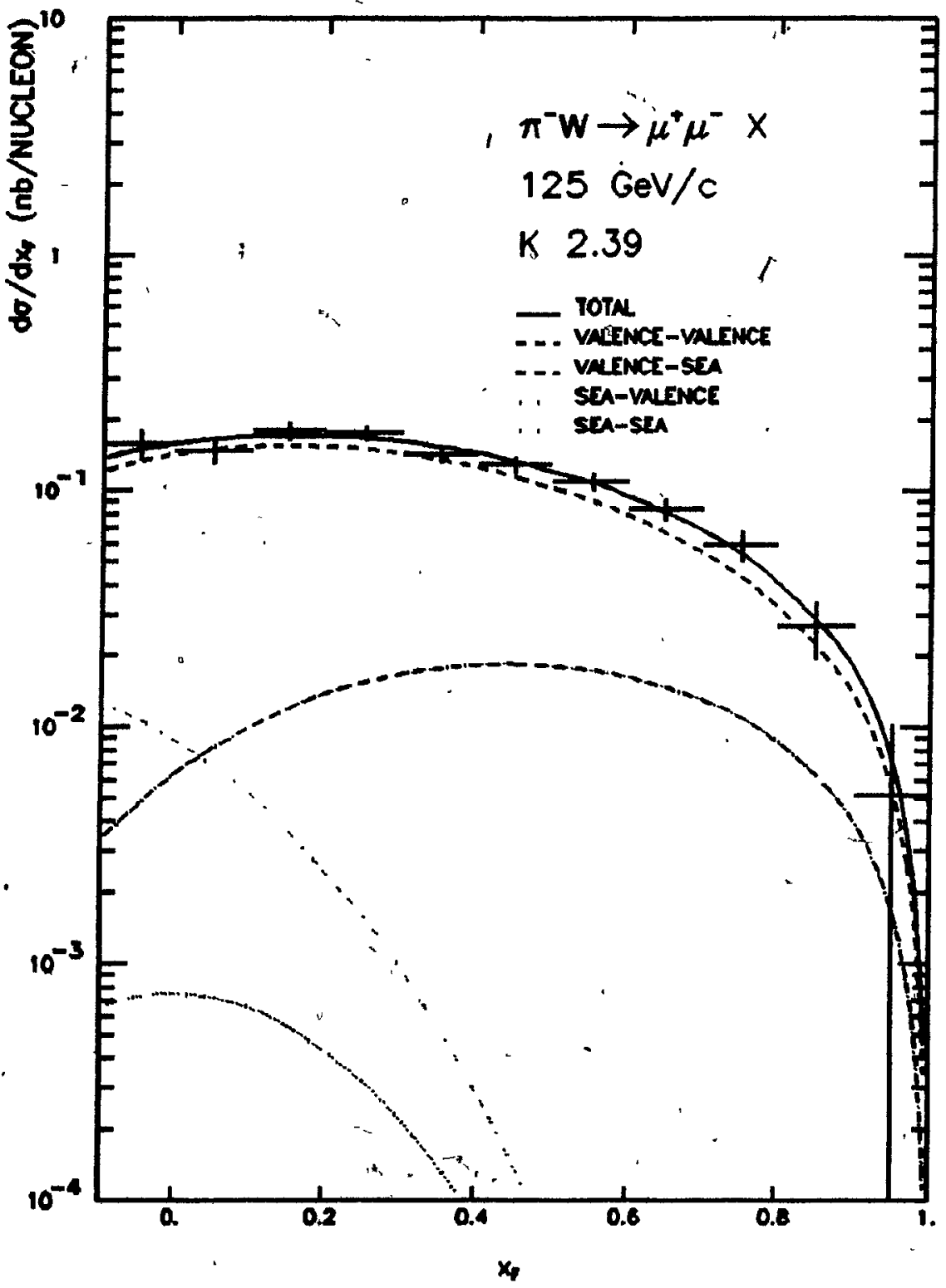

Figure $44^{\text {- }}$ - Pion $x_{F}$ Distribution Compared To Drell-Yan Predictib

The points show the $x_{F}$ distribution of the pion produced data. The solid line shows the shape of the cross section predicted by the Drell-Yan model using structure functions from measurments of deep-inelastic scattering for the nucleon, and our fits for the pion structure function. The curve has been multiplied by a factor of 2.39 to reproduce the measured total cross section for $4.0 \leq M \leq 9.0 \mathrm{GeV} / \mathrm{c}^{2}$. with $\mathrm{x}_{F} \geq 0$. The other curves show the components of the predicted cross section as indicated.

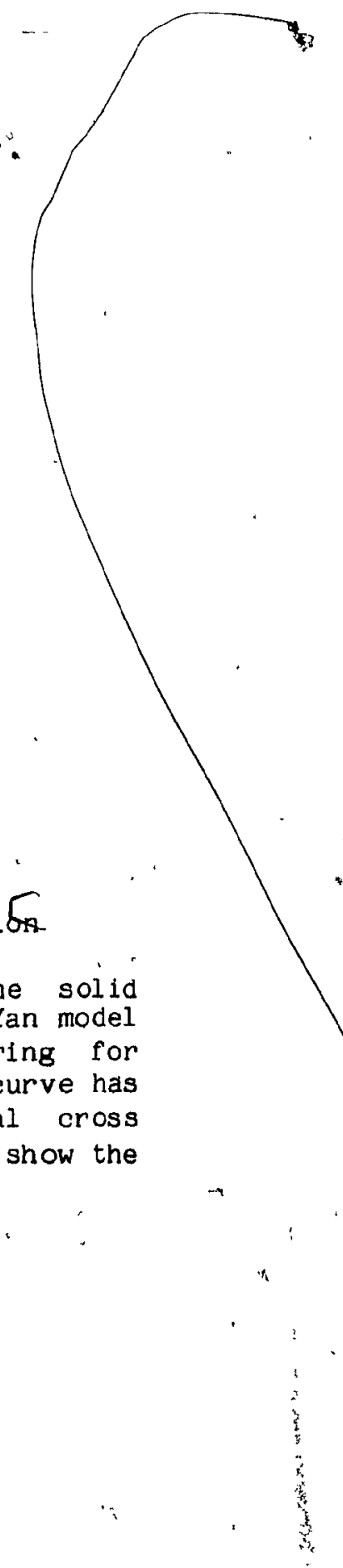



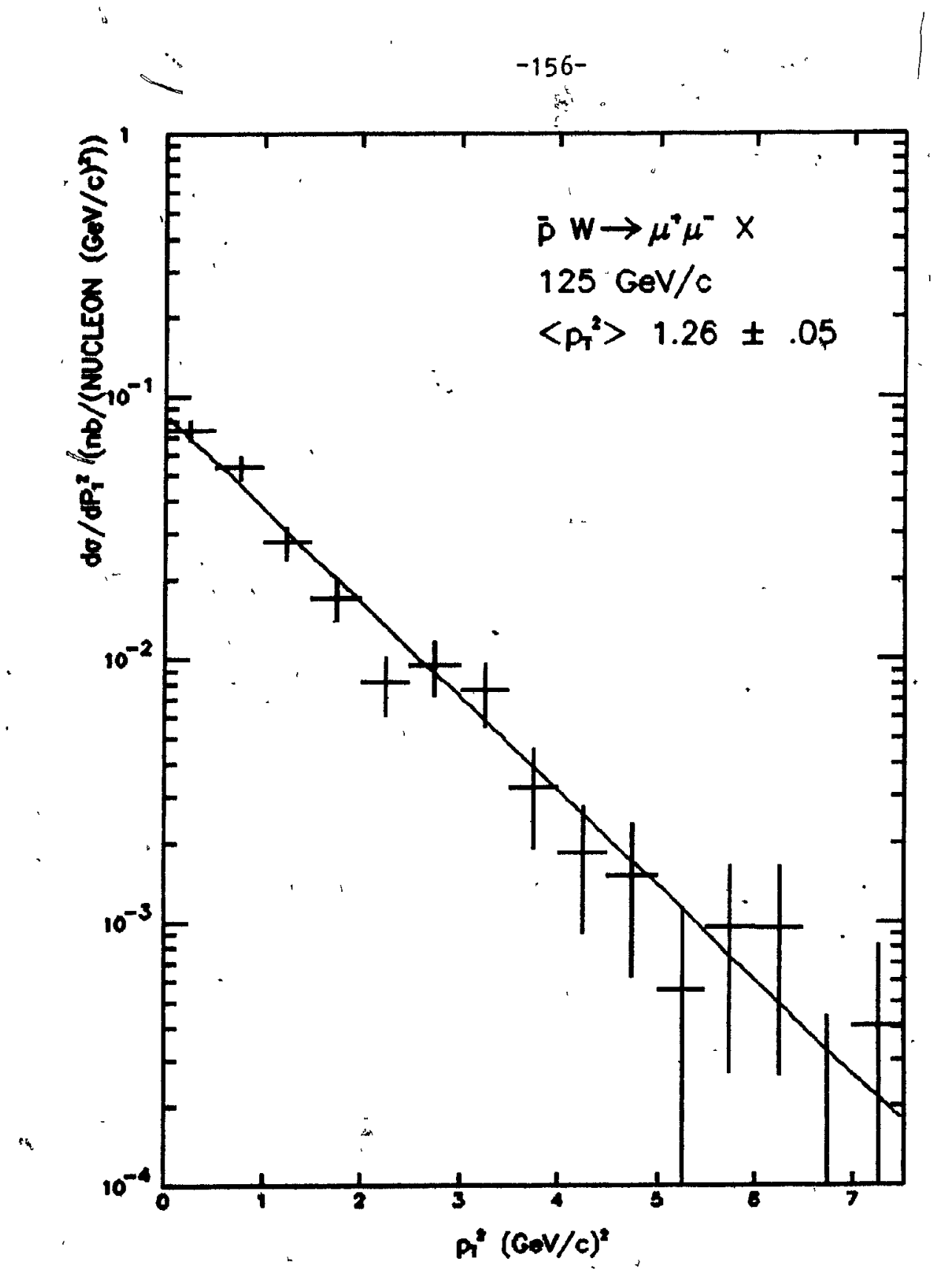

Figure 45 - Antiproton $\mathrm{p}_{\mathrm{T}}{ }^{2}$ Distribution

The points show the differential cross section, $d o / d p^{2}$, for the production of muon pairs in antiproton-tungsten collisions. The curve shows the Gaussian $\mathrm{f}$ it to the data discussed in the last chapter using the parameter value of $P_{T_{0}}=1.117$ given in Table 7 . 
$4 \leq M \leq 9 \mathrm{GeV} / \mathrm{c}^{2}$ and $\mathrm{x}_{\mathrm{F}} \geq 0$ are shown as a function of $\mathrm{p}_{\mathrm{T}}{ }^{2}$ in Figure 45 and Figure 46 with the Gaussian distributions used to caloulate the acceptance. In both cases the 11 ts are very good, and the parameters of the Gaussians are approximately the same for the two beam particles.

The $\cos \theta$ distribution for antiproton events with $M$ between 4 and $9 \mathrm{GeV} / \mathrm{c}^{2}$ and. With $x_{F} \geq 0$ is shown in Figure 47 and the pion cos $\theta$ dis.tribution is shown in Figure 48. The curves drawn on the flgures are the $1+\cos ^{2} \theta$ distributions assumed to calculate the acceptance. The lfmited acceptance of the spectrometer at high values of $\cos ^{2} \theta$, and the small data sample make it difficult to determine the value of the $\lambda$ parameter. The value of $\lambda=1.06 \pm 0.28$ obtalned from the $f$ it to the pion produced data is consistent with the Drell-Yan model so that we have assumed that $\lambda=1$ for the antiproton produced data as well when fitting the other varlables. While the results from fitting with MINUIT discussed in the last chapter suggest that the $\cos \theta$ distribution may be somewhat flatter than this, the error is large and the data is not inconsistent with the assumed distribution.

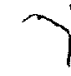

The $\phi$ distributions are shown in Figure 49 and Figure 50. These distributions were assumed to be uniform in calculating the acceptance for the other variables, as would be expected from the Drell-Yan model. Both the antiproton and the plon data may show a weak dependence on $\cos 2 \dot{\phi}^{\circ}$, but this is not statistically signifloant and will not af fect the distributions integrated over this variable.

The good agreement between the data and the parameterizations chosen to describe each of the kinematic distributions leads to confidence in the accuracy of the acceptanoe calculations for the remaining variables. 
$-158-$

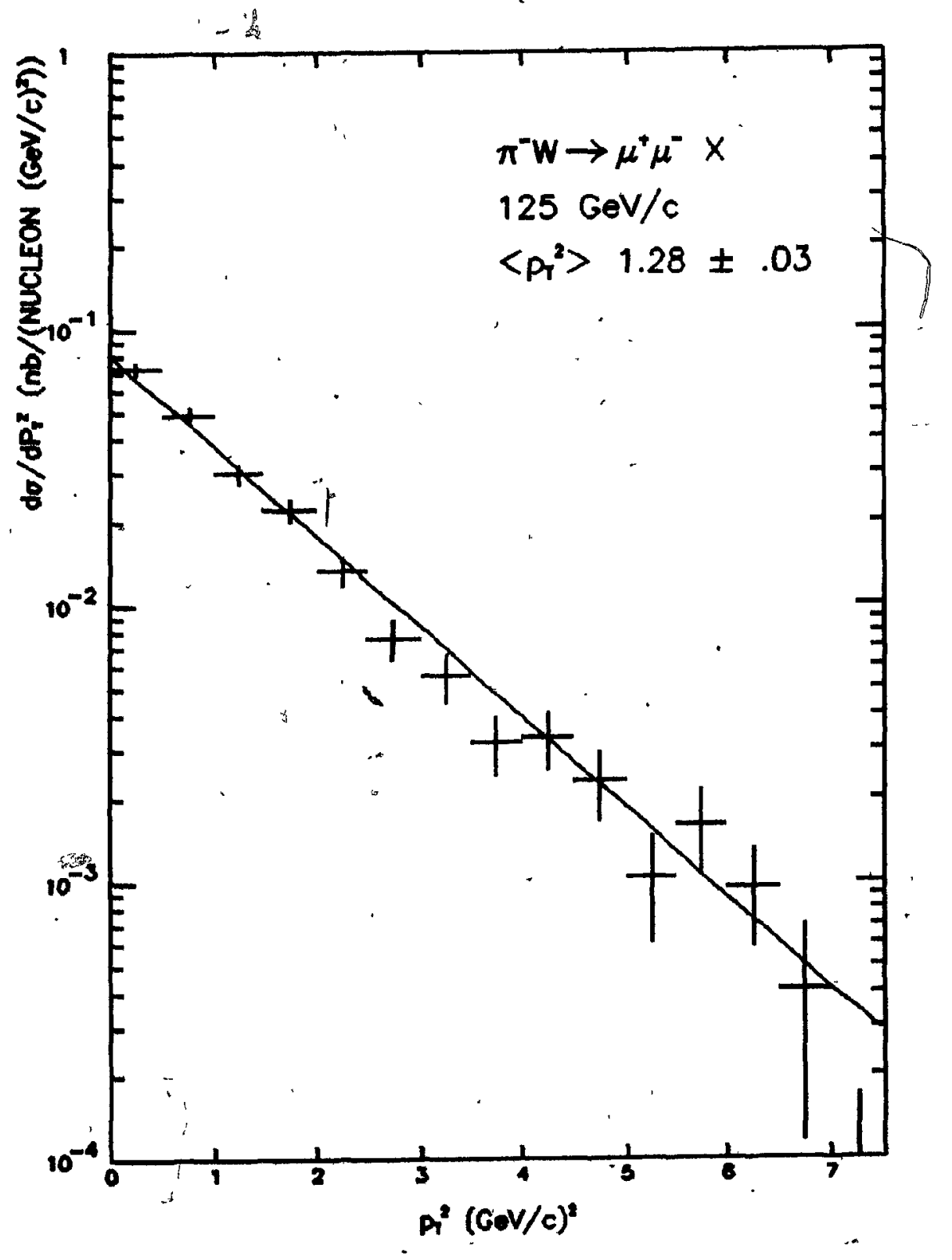

Figure 46 - Pion $\mathrm{p}_{\mathrm{T}}{ }^{2}$ Distribution

The points show the differential cross section, do/dp ${ }^{2}$, for the production of muon pairs in pion-tungsten collisions. The curve shows the Gaussian fit to the ,data discussed in the last chapter using the parameter vaiue of $\mathrm{P}_{\mathrm{T} 0}=1.155$ given in Table 7 . 
$-159-$

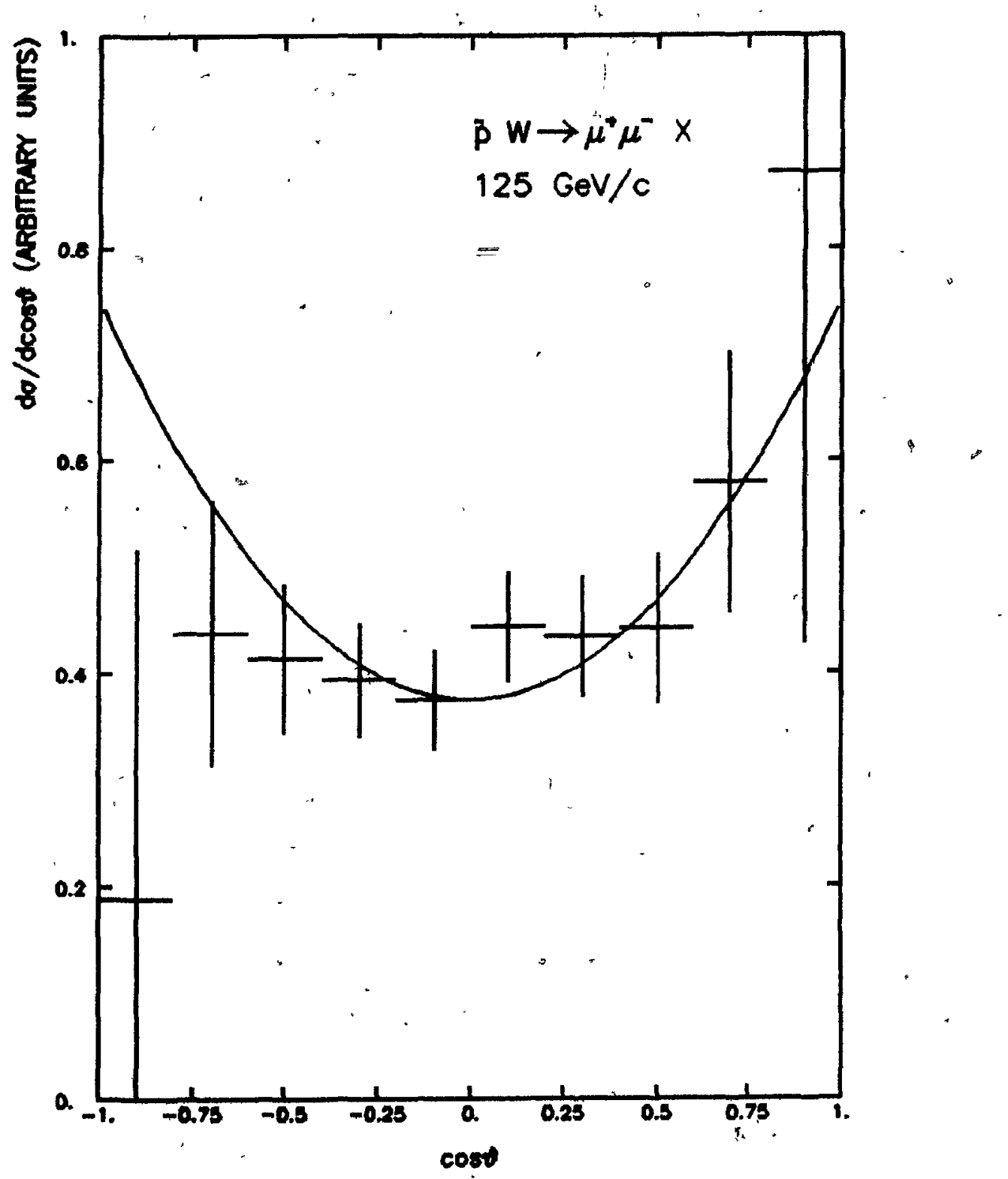

Figure 47 - Antiproton $\cos \theta$ Distribution In The Gottfreid-Jackson Frame

The points show the distribution, $0^{-1} d \sigma / d \cos \theta$, of muon pairs produced in antiproton-tungsten collisions. The curve shows the $1+\cos ^{2} \theta$ distribution assumed when caluculating the acceptance and discussed in the last chapter. . 


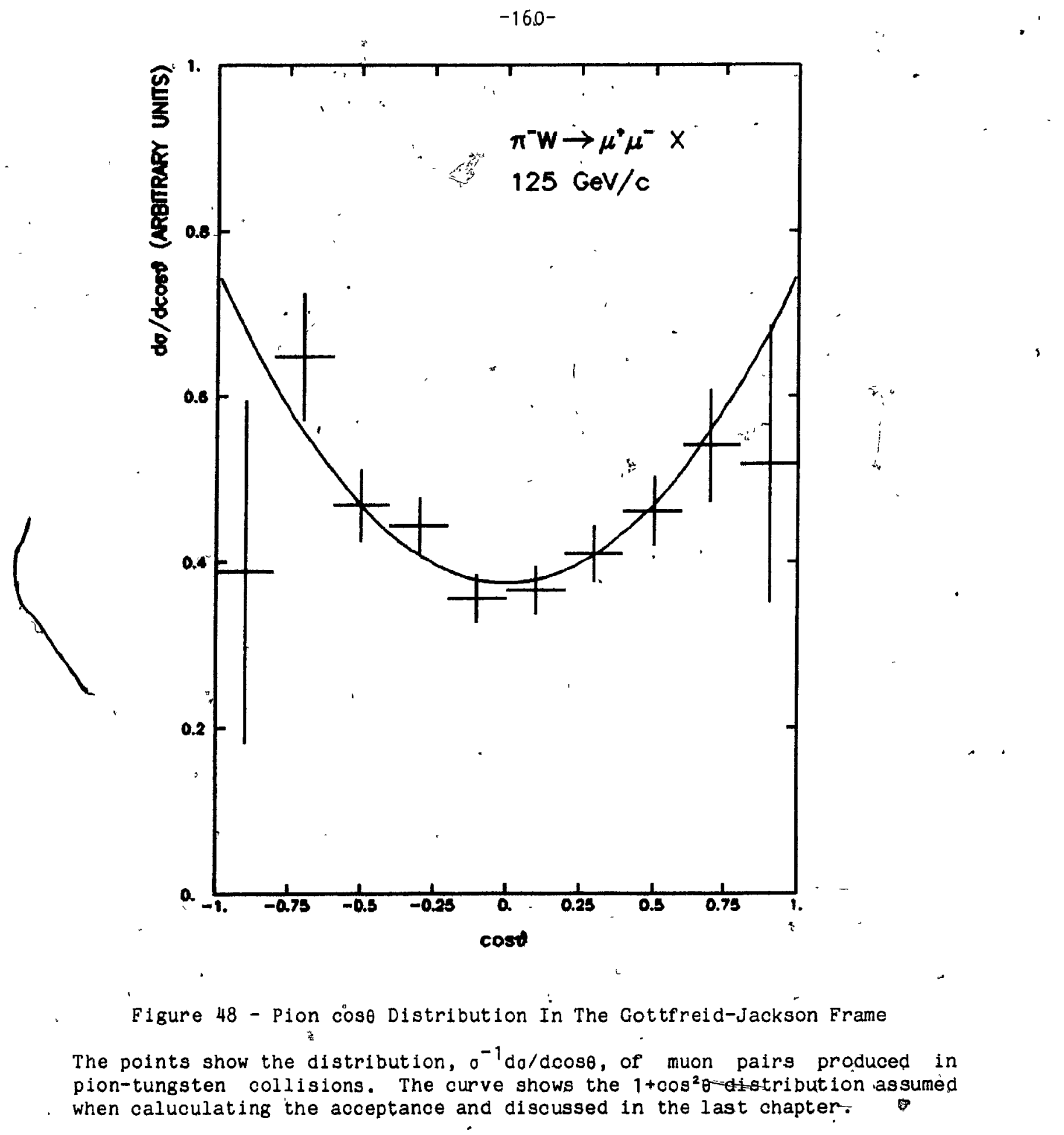




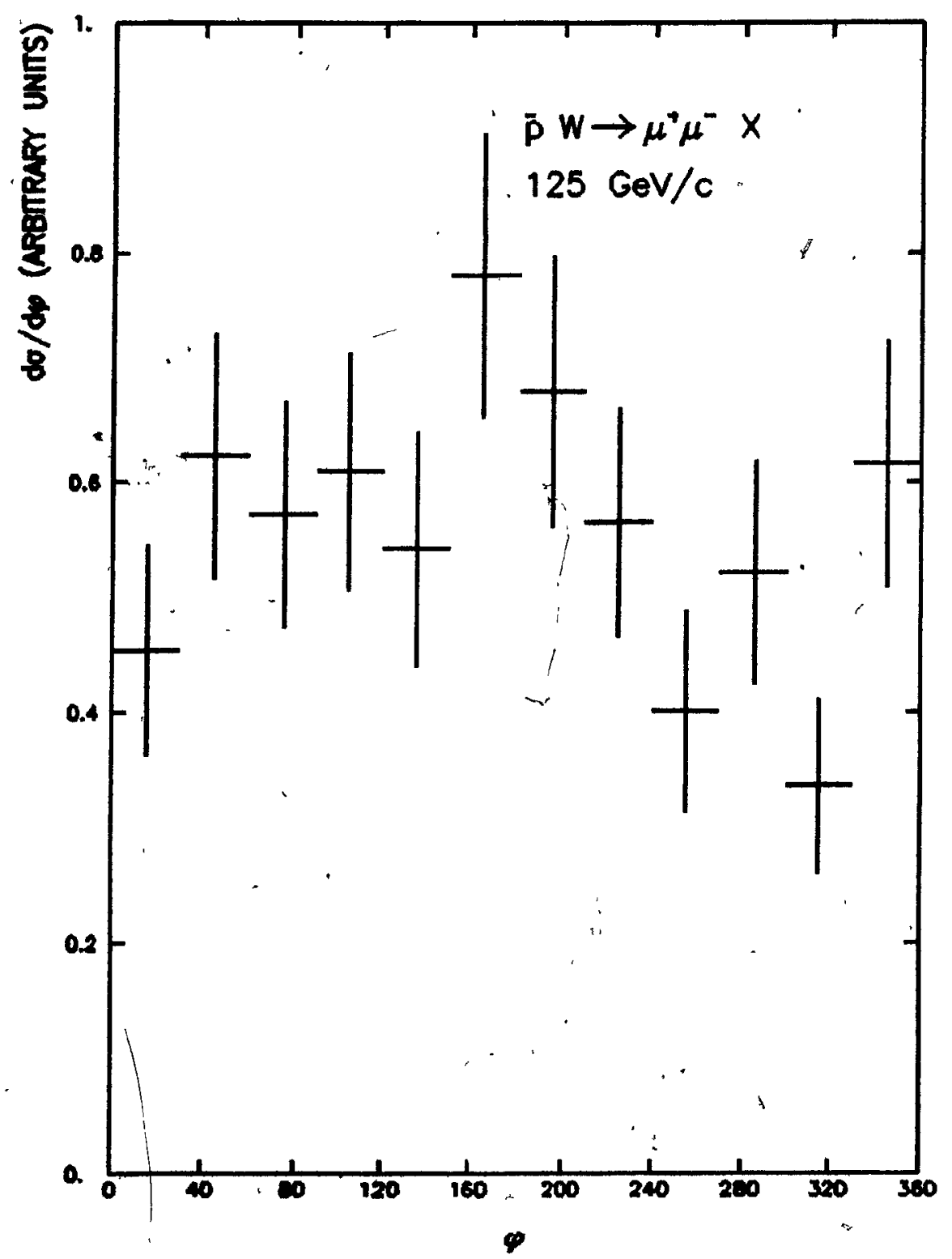

$\equiv$

Figure 49 - Antiproton $\phi$ Distribution In The Gottfreid-Jackson Frame

The points show the distribution, $o^{-1} d \sigma / d \phi$, of muon pairs produced in antiproton-tungsten collisions. This distribution was assumed to be uniform when the acceptance of the spectometer was calculated. 


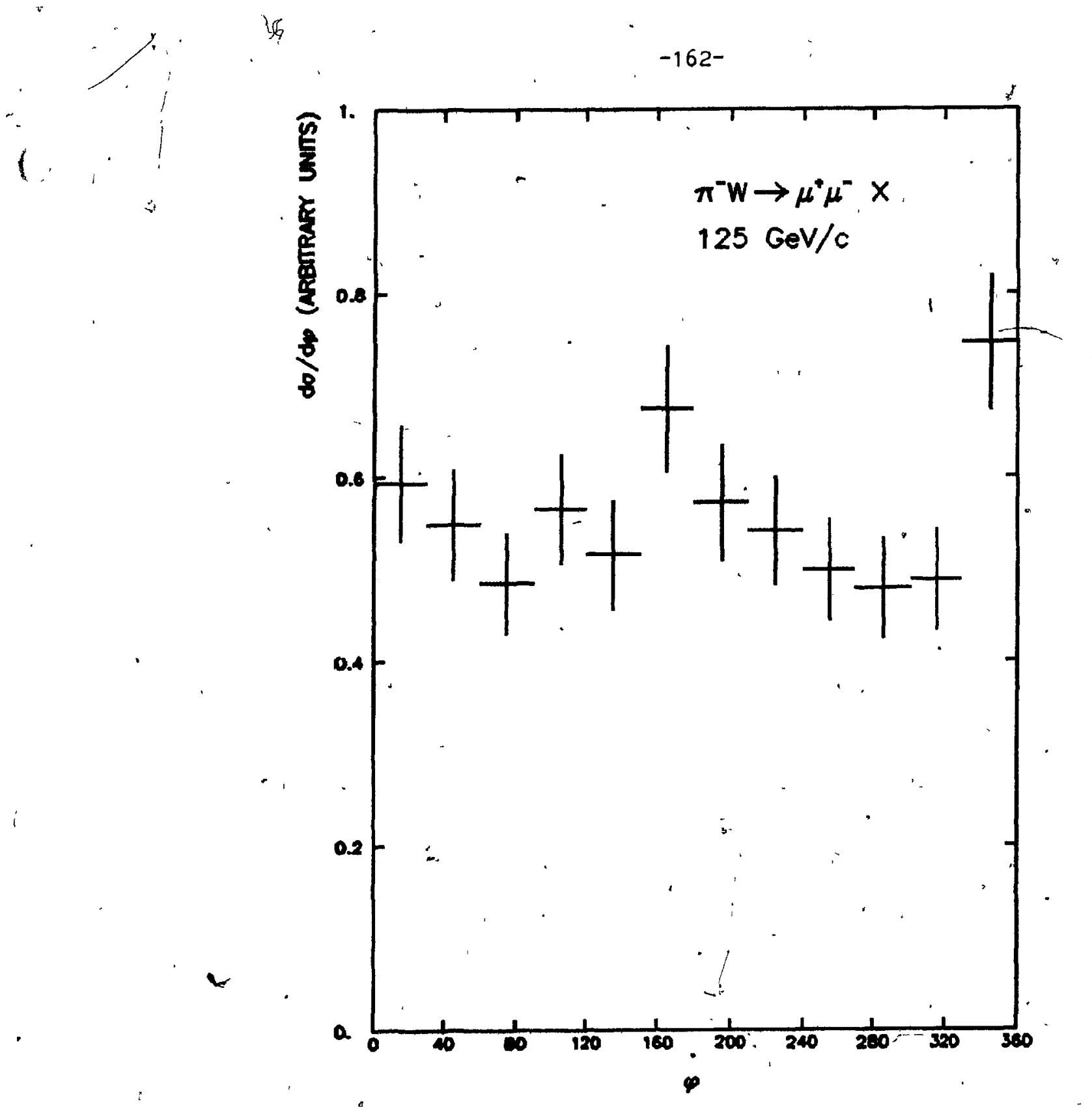

算 


\subsection{Comparisons With Other Experiments}

Comparison of the data with other experiments is of interest from two points of 'vlew. Scaling behavlour of the cross sections provides confirmation of the pointlike nature of the interacting constituents while comparisons with the higher statistics plon data of other experiments provides a valuable cross-check on the antiproton results.

Figure 51 shows the scaling cross section, $M^{3} \frac{d o}{d M}$, as a function of $\sqrt{\tau}$ for antiproton produced events $w^{m}$ th $x_{F} \geq 0$ compared with similar data using a $150 \mathrm{GeV} / \mathrm{c}$ beam obtained by the NA3[126] experiment at CERN. Both the dependence on $\sqrt{ } \tau$ and the magnitudes of the two data sets agree very well. Pion produced events are 'shown In Figure 52, and compared with points from four other experiments[127][128][43][44]. As most fixed target experiments have limited acceptance in the backward hemisphere, the extrapolation of the cross section to all $x_{F}$ is subject to considerable systematic errors. We have chosen to restrict our results to the reglon of $x_{F} \geq 0$, but the same overall trend of the data is evident from the results of all the experiments. Note that we have multiplied the CIP points for this plot by a factor of

$$
A_{W}^{0.12}=1.87^{\circ}
$$

to reflect our assumed A dependence, rather than the A 1.12 dependence used In their analysis[39].

Perhaps a better test of scaling 1s the comparison of the cross sections, $s^{3 / 2} \cdot \frac{d 0}{d M}$, for the region with $x_{F} \geq 0$ as shown in Figure 53. Our plon data is compared with data from the CIP[128] and Omega[43] groups. All of the Omega data and the bulk of the CIP data were taken with a tungsten 


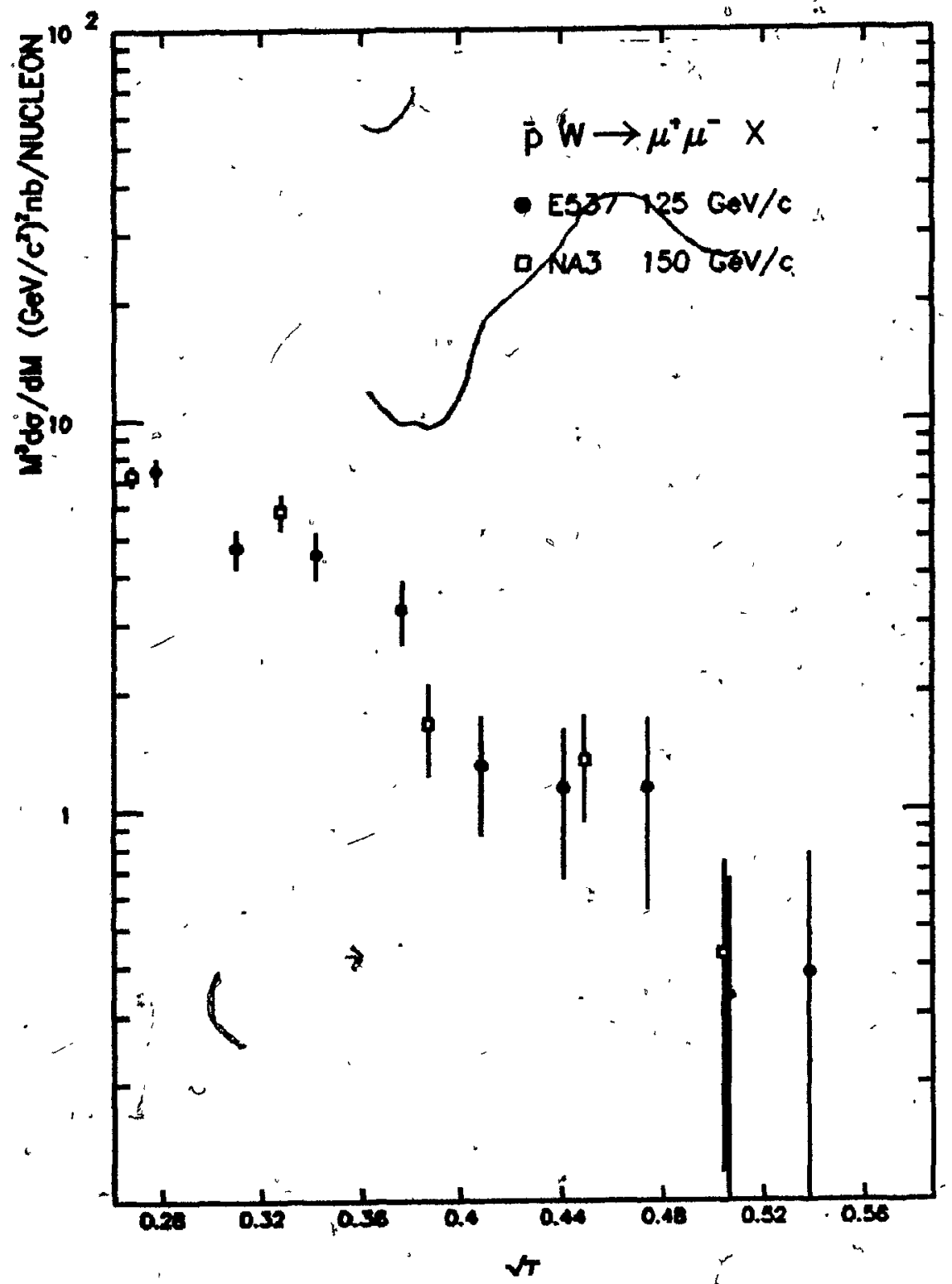

Figure 51 - Antiproton Scaling Cross Section Compared With NA3

Our measurement of the cross section, $\mathrm{M}^{3} \mathrm{do} / \mathrm{dM}$, for the production of muon pairs with $x_{F} \geq 0$ in antiproton-tungsten collisions is shown together with data obtained by the NA3[10] collaboration using a $150 \mathrm{GeV} / \mathrm{c}$ beam incident on a platinum target, as a function of: $\checkmark \tau$. 


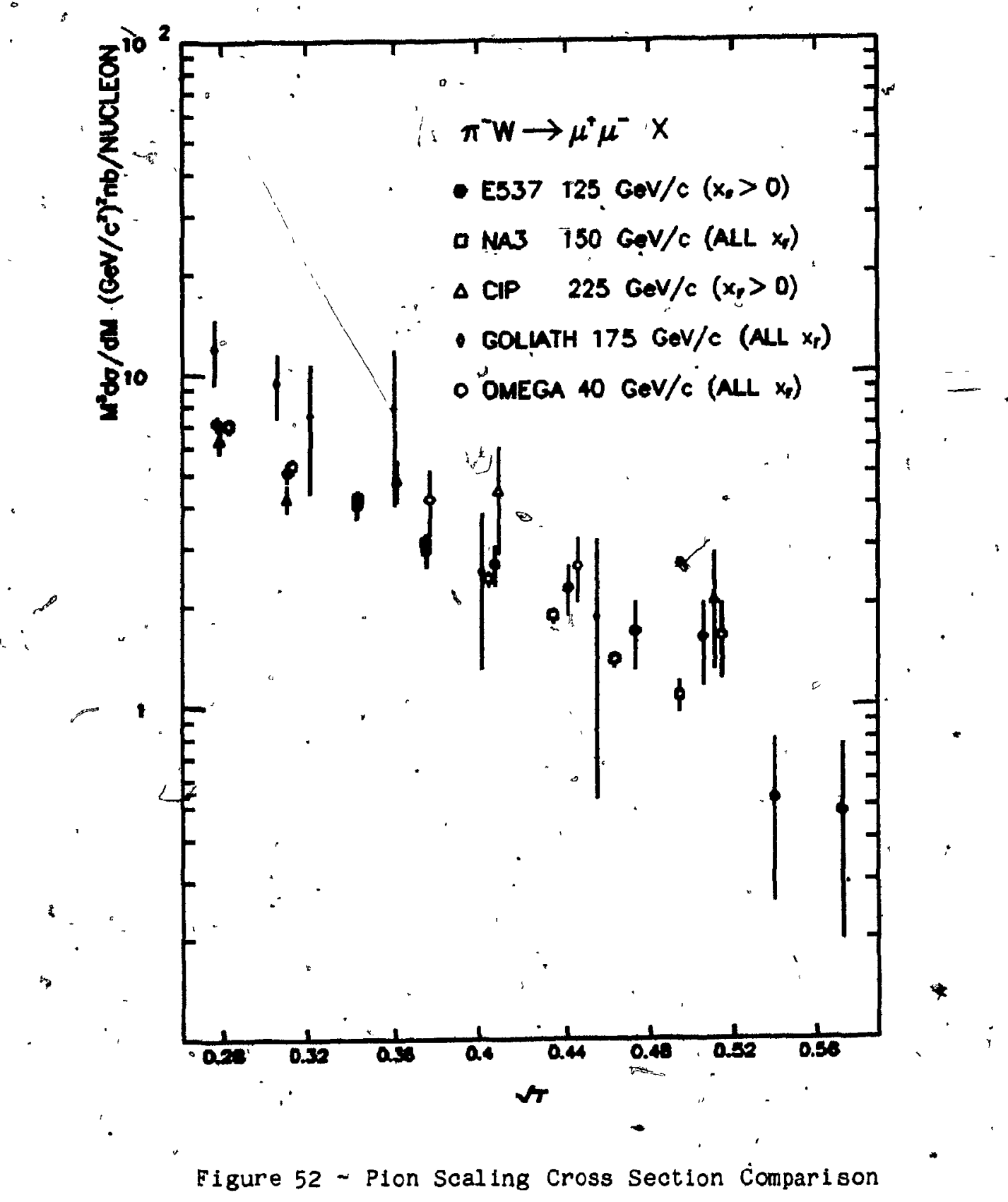

Our measurement of the cross section, $M^{3} \mathrm{do} / \mathrm{dM}$, for the production of muon pairs in pion-nucleus collisions is shown together with data obtained by the NA3 [46], CIP[128], Gollath[44], and Omega[43] collaborations. While we have - restricted our measurement to the region with $x_{F} \geq 0$, the same overall trend is apparent in all the data. 

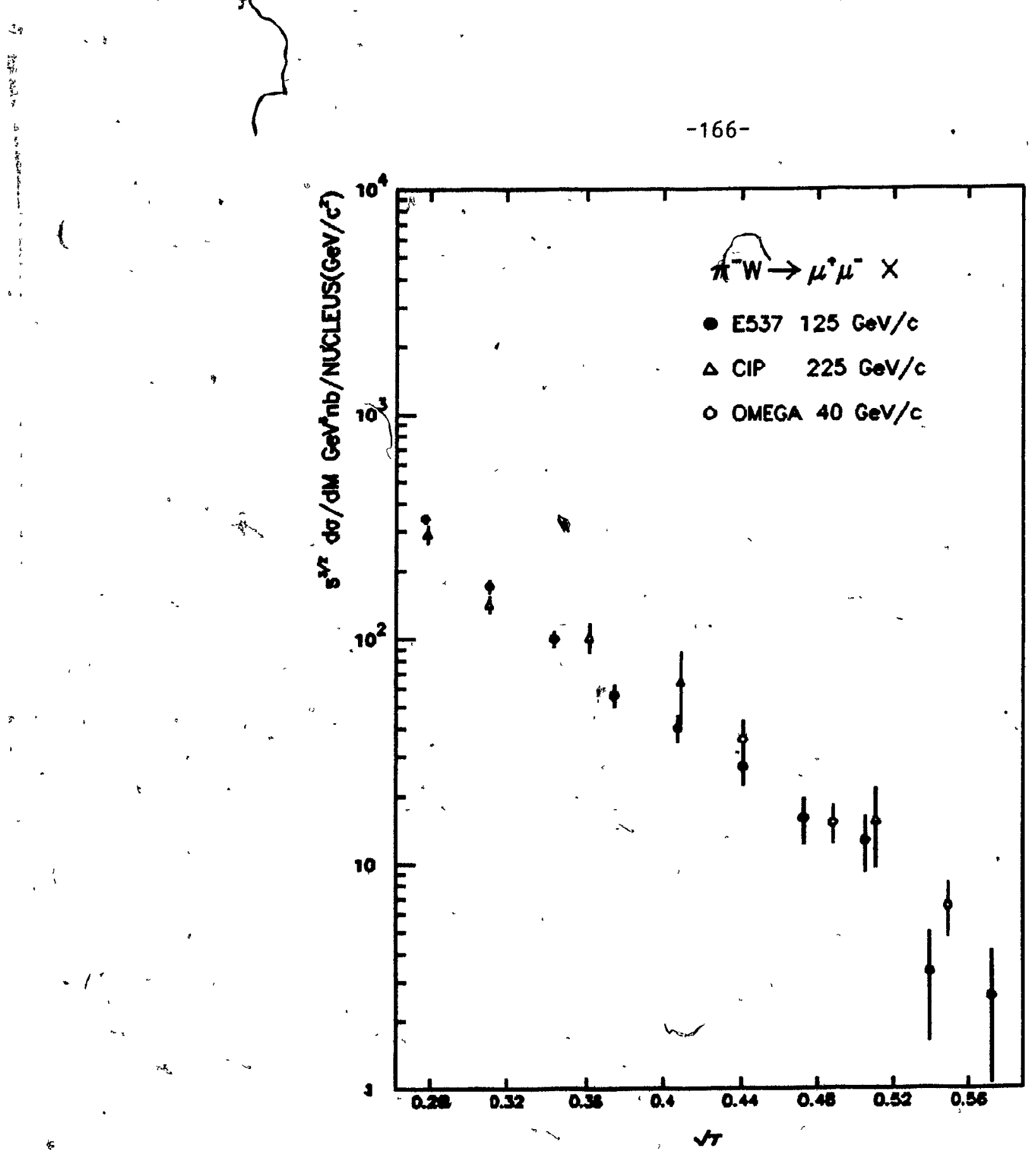

Figure 53 - Pion Mass Cross Section Comparison

Our measurement of the scaling cross section, $s^{3 / 2} \cdot d o / d M$, with $x_{F} \geq 0$, for the pkoduction of muon pairs in pion-tungsten collisions is shown together with data obtained by the CIP[128] and Omega[43] collaborations as a function of $\checkmark \tau$. 
target, so that we have presented these cross sections per tungsten nucleus td avoid any controversy about A dependence. There is good agreement over a wide range of incident beam momenta.

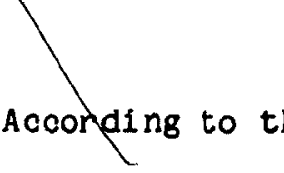

exhiblt scaling behaviour if it' is integrated over the same $\tau$ region. Figure 54 shows the good agreement in both shápe and magnltude between our, measurement of the cross section $s \cdot \frac{d a}{d x_{F}}$ for masses between 4.10 and $6.71 \mathrm{GeV} / \mathrm{c}^{2}$ using a $125 \mathrm{GeV} / \mathrm{c}$ pion beam incident on a tungsten target and (v) similar data for masses between 5.5 and $9.0 \mathrm{eV} / \mathrm{c}^{2}$ as measured by the CIP experiment using a $225 \mathrm{GeV} / \mathrm{c}$ beam[128]. Again these cross sections are given per tungsten nucleus.

\subsection{Structure Functions}

The distributions of the data points in the $x_{1}-x_{2}$ plane are shown in Figure 55 for antiproton produced events and in Figure 56 for pion produced events. Lines of constant and $x_{F}$ for a beam momentum of $125 \mathrm{GeV} / \mathrm{C}$ are also shown. The kinematic cut at a mass of $4.0 \mathrm{GeV} / \mathrm{c}^{2}$, which is required to eliminate the resonance region, and our limited acceptance for $x_{F} \leq$. make us insensitive to the behaviour of the beam structure functions below $x_{1}=0.2$. The kinematic cut at $4.0 \mathrm{GeV} / \mathrm{c}^{2}$ is not sharply defined in the $x_{1}-x_{2}$ plane because of the sintte momentum bite of the beam and events can appear below the line.

.9

The antiproton and pion structure functions can be extracted from the unbinned data using the fitting procedure outlined in the last chapter. The

' antiproton valence structure functions were parameterlzed as

$$
1 .
$$

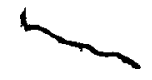




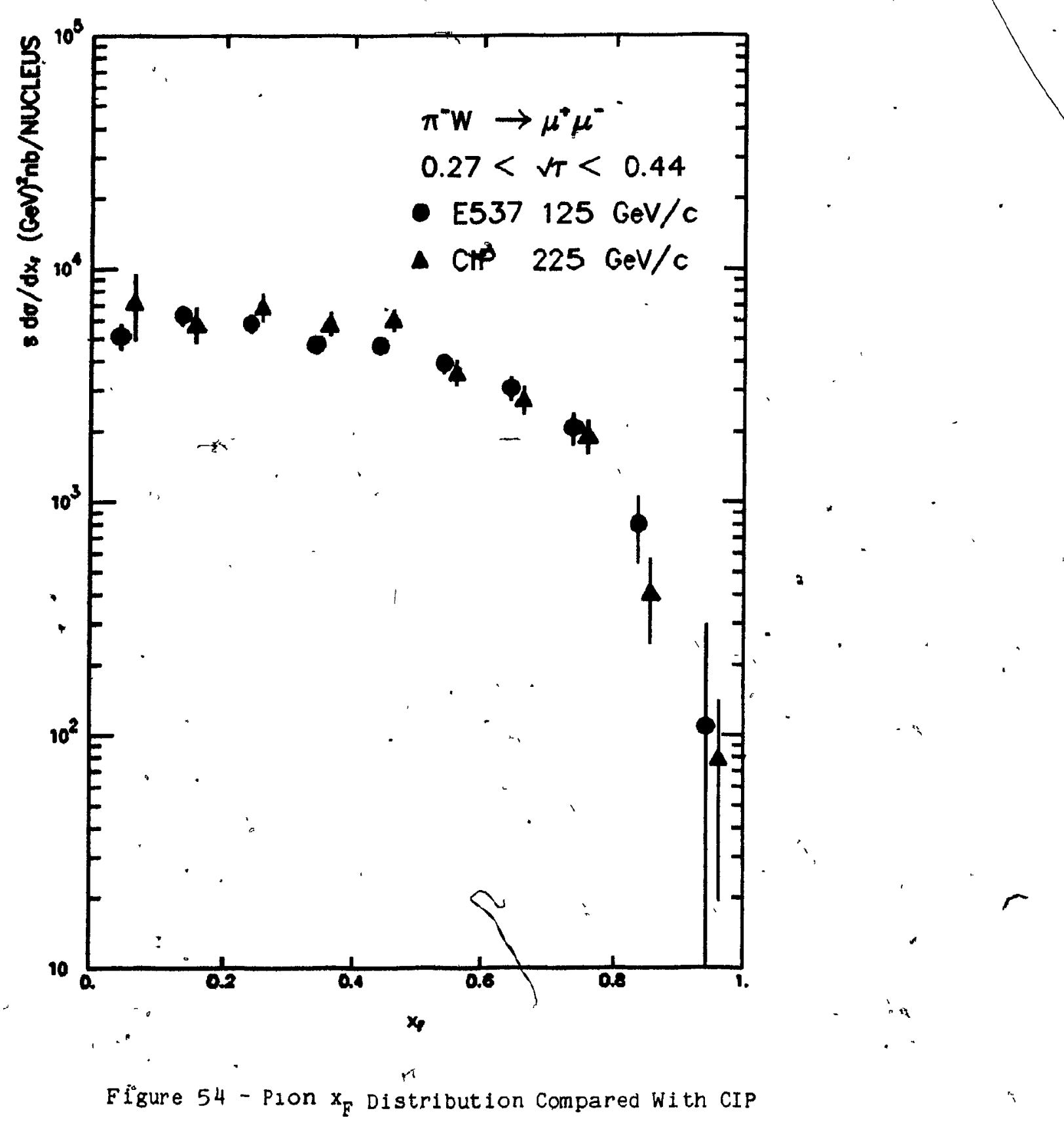

Our measurement of the cross section, $s \cdot d_{0} / d x_{F}$, for $4.10 \leq \dot{M} \leq 6.71 \mathrm{GeV} / c^{2}$ is compared to data obtained by the CIP collaboration[128] in the same region of $\checkmark \tau$ for the production of muon pairs in pion-tungsten collisions. 


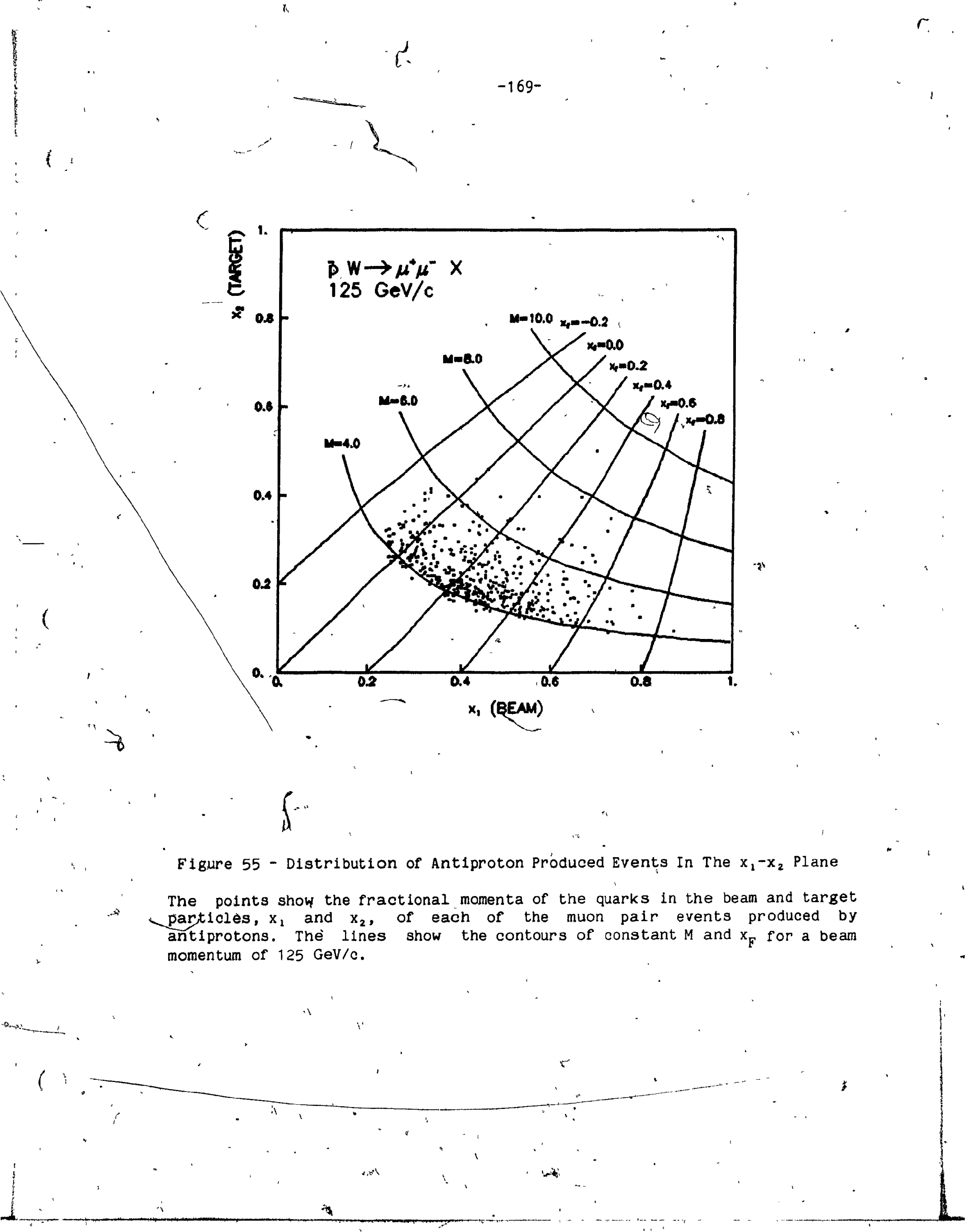




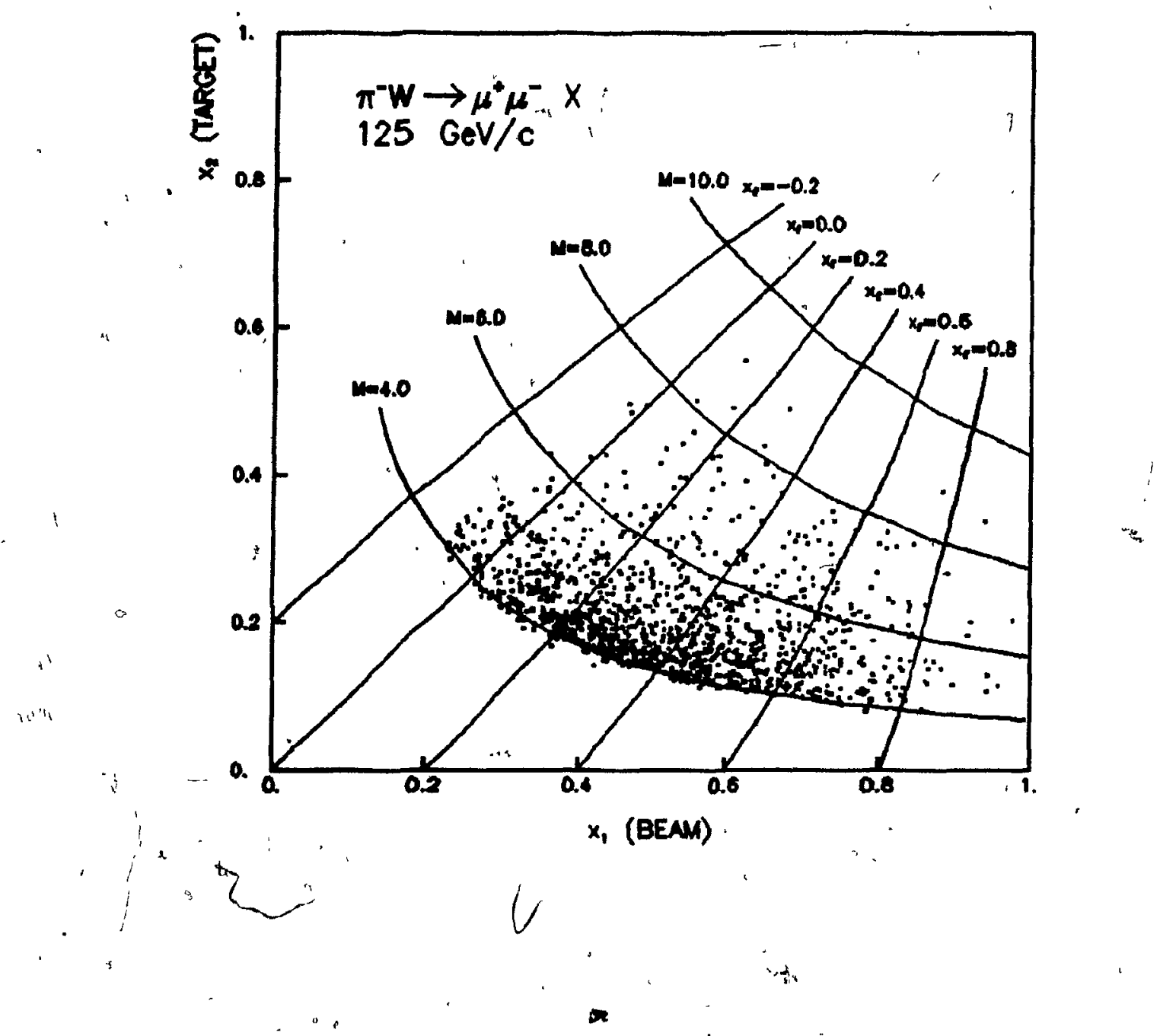

Figure 56 - Distribution of Pion Produced Events In The $x_{1}-x_{2}$ Plane.

The points show the values of $x_{1}$ and $x_{2}$ of each of the muon pair events produced by plons. The lines show the contours of constant $M$ and $x_{F}$ for. $a$ beam momentum of $125 \mathrm{GeV} / \mathrm{c}$. 


$$
\bar{u}_{v}(x)=A_{A}^{\alpha}(1-x)^{\beta},
$$

and

$$
\bar{d}_{v}(x)=0.57(1-x) \bar{u}_{v} \text {. }
$$

with the requirement that

$$
\int_{0}^{1}\left[\bar{u}_{v}(x)+\bar{d}_{v}(x)\right] \frac{d x}{x}=3 \text {. }
$$

The sea terms used were taken from the analys1s by Duke and Owens[113]. With these structure functions;-the Drell-Yan model was used to predict, the joint $M-x_{F}$ probability distribution

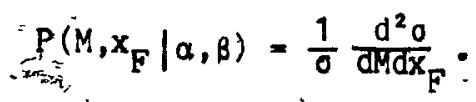

The width of the $\dot{p}_{\mathrm{T}}$ distribution was allowed to vary freely, while the cose distribution in the Gottfreid-Jackson frame was. again-assumed to be $1+\cos ^{2} \theta$

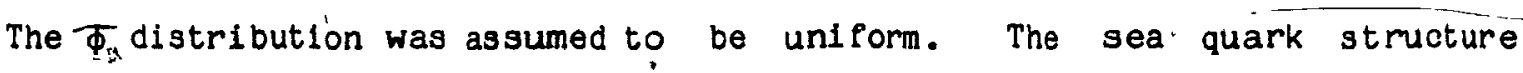
functions of the antiproton were taken to be the same as the proton sea antiquark structure functions, by particle-antiparticle symmetry. The neutron structure functions were obtalned from, the proton structure functions by isospin rotation. The results obtained under these conditions are given ąs Set 2 of Table 13.

When these parameter values are used, the Drell-Yan model requires a $K$ factor of

$$
\mathrm{K}_{\overline{\mathrm{p}}}^{*}=1.91
$$

to reproduce the experimental cross section. The kinematic cuts at $M=4.0 \mathrm{GeV} / \mathrm{c}^{2}$ and $\mathrm{x}_{F}=-0.1$ eliminate the region of the $x_{1}-x_{2}$ plane with $x_{1} \leq 0.2$, making it difficult to determine a reliably; therefore the structure functions were refit with a flxed to $0.5^{\circ}$ as expected from Regge theory arguments[129]. The results obtained with this constraint are given as Set 1 of Table 13. The $\mathrm{K}$ factor for antiprotons with these assumptions $1 \mathrm{~s}$ 
Table 13 - Structure Function Parameters

$\because$

Table 13 - structure Function Parameters

This table presents the results of fitting the antiproton and pion valence structure functions to the mathematical form $x^{\alpha}(1-x)^{\beta}$ under various assumptions about the sea and nucleon valence structure functions. The values of $\alpha$ and $\beta$ obtained from the fits are shown together with the error bounds estimated by MINUIT. The $K$ factor obtained under each of the assumptions is also given.

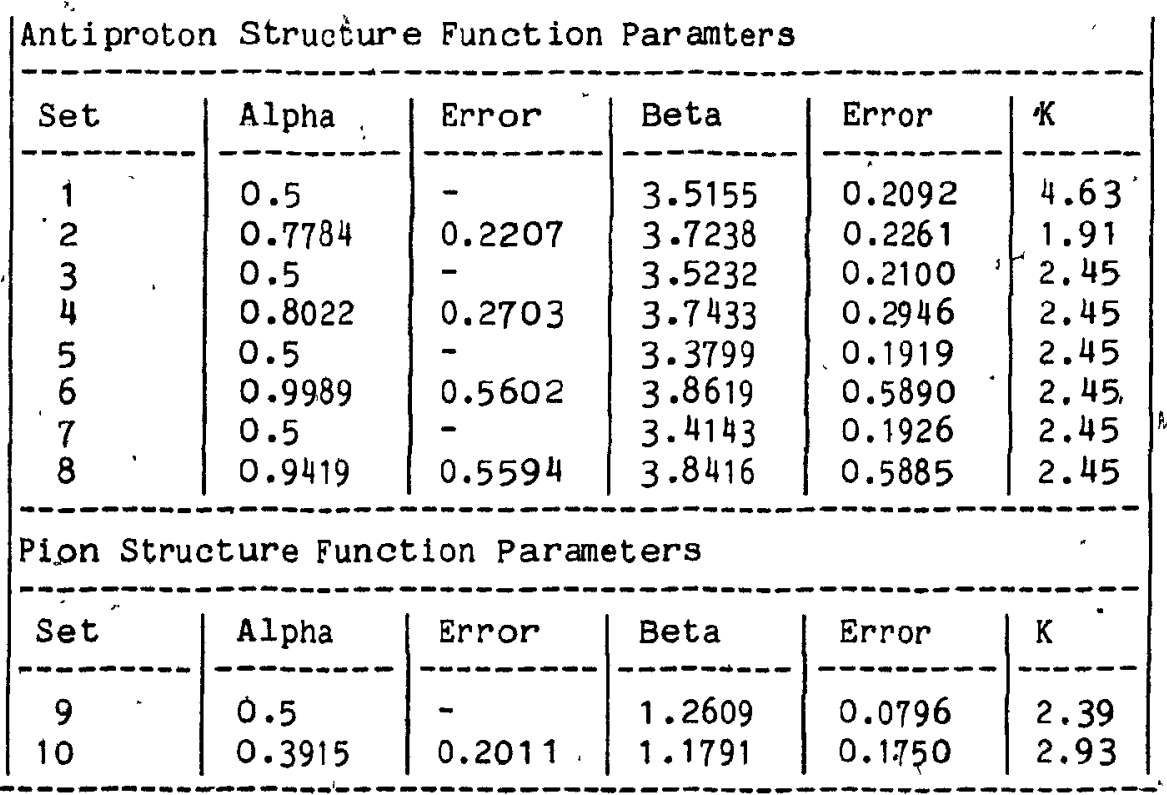


$\therefore K \bar{p}=4.63$

and this illustrates the sensitivity of the cross section to the assumed behaviour of the structure functions at low values of $x$. Approximatel $y$ one half thê integral,

$$
\int_{0}^{1}\left[\bar{u}_{v}(x)+\bar{d}_{v}(x)\right] \frac{d x}{x}=3 \text {. }
$$

is contributed by the region $x \leq 0.2$. The normalization of the Drel1-Yan cross section in turn depends quadratically on the normalization of the structure functions. The choice of a "different functional form to parameterize the structure functions, for example, a sum of tierms of the form $x^{\alpha}(1-x)^{\beta}$, could drastically alter the normalization of the calculated Drell-Yan cross section whlle still conserving baryon number by integrating to glve three valence quarks.

The relative contribution of the valence quarks is also sensitive to the method used to normalize the structure functions. Using the Drell-Yan calculations with deep inelastic structure functions as a guide, the data was constrained so that the valence quarks contributed a constant fraction of the total cross section given by

$$
\sigma_{v v}=0.91 \text { Total: }
$$

The results of these fits with a fixed, (Set 3), and a free, (Set 4), are also given. In Table 13. It can be seen from the small changes in $\alpha$ and $\beta$, that the shape of the structure. function is not very sensitive to the normalization convention chosen. In addition, the antiproton structure function was fit using the deep inelastic structure functions for the target particle only. While we did not have sufficient data to allow the beam particle structure functions to violate scaling, we fllowed the target particle structure functions to evolve with $Q^{2}=M^{2}$, and the results are given as Sets 5 and 6 in Table 13. Using the deep Inelastic structure 
functions for the target particle with $Q^{2}$ fixed to our mean value of $\left\langle M^{2}\right\rangle=25\left(\mathrm{GeV} / \mathrm{c}^{2}\right)^{2}$ glves similar results which are sets 7 and 8 of $\mathrm{Tab}$ ie 13.

- The pion valence structure function was $f$ it in a similar fashion. The valence structure function was parameterized as

$$
V^{\pi}(x)=A x^{\alpha}(1-x)^{\beta}
$$

normalized such that.

$$
\int_{0}^{1} v^{\pi}(x) \frac{d x}{x}=1 .
$$

The NA3[130] result,

$$
S^{\pi}(x)=0.292(1-x)^{8.2}
$$

obtained from a simultaneous analysis of $200 \mathrm{GeV} / \mathrm{c}^{+}$and $\pi^{-}$daṭa, was used for the sea. Results with a fixed at 0.5 and free to vary are given as Sets 9 and 10 respectively in Table 13.

Using the results of Set 9 , where $a$ has beeh fixed at 0.5 , in the Drell-Yan model, we find a $K$ factor of

$$
K_{\pi^{-}}=2.39
$$

is needed to reproduce the experimental cross section for the pion produced data with masses between 4.0 and $9.0 \mathrm{GeV} / \mathrm{c}^{2}$ and $x_{F} \geq 0$. Using the results of Set 10, where the value of $\alpha^{*}$ is determined by the fitting programme, a $K$ f'actor of

$$
K_{\pi}=2.93
$$

is required. As for the antiprotion, the normalization of the structure function is doninated by its behaviour at low values of $x$. Using a different functional form to parameterize the structure function results in a different $K$ factor. It is apparent from the results of the plts to both the antiproton and the pion produced data that the sum rules are not.a good 

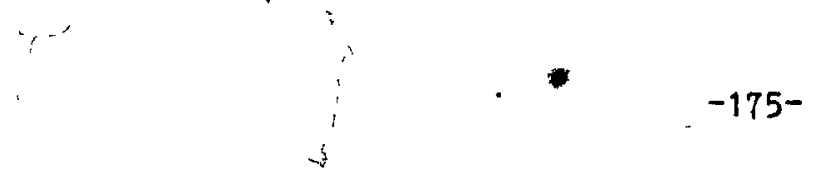

gulde to the normalization of the structure functions in the absence of information about their behaviour at low values of $x$. The shape of the structure functions is less sensitive. The results of two of our antiproton structure function fits are shown in Figure 57 . The points on this graph are projected from our data as discussed in the next section. The dashed line shows the results of set 5 of Table 13, where $\alpha$ was fixed to 0.5 , while the dot-dashed line shows the results of Set 6. Both of these fits desoribe the data well over the range of $x_{1}$ accessible to our experiment. The dot-blank $l$ ine and the dotted line show the average deep inelastic valence structure functions with $Q^{2}=M^{2}$ and with $Q^{2}=\left\langle M^{2}\right\rangle=25\left(\mathrm{GeV} / 0^{2}\right)^{2}$, respectively. All of the curves have been multiplied by

$$
K=2.45
$$

to allow them to be compared to the data points. It is apparent from these curves that the agreement between our structure function fits and the "results obtained "fron deep inelastic scattering is good. The small differences between the two deep inelastic curves justify ignoring the soale breaking behaviour of the beam structure function in the fitting.

Figure 58 shows our fits to the pion valence structure function. Set 9 of Table 13 is shown by the dashed line, and Set 10 is shown as the dot-dashed line. Again the points are the projection of our data on the $\cdot x_{1}$ axis described in the next section, and the two fits have been multipl ied by their respective $K$ factors. Both reproduce the shape of the structure function very well. It is clear that any measurement of the plon $K$ factor is subject to large systematic uncertalnties as long as the behaviour of the valence quark structure function is unkown. 


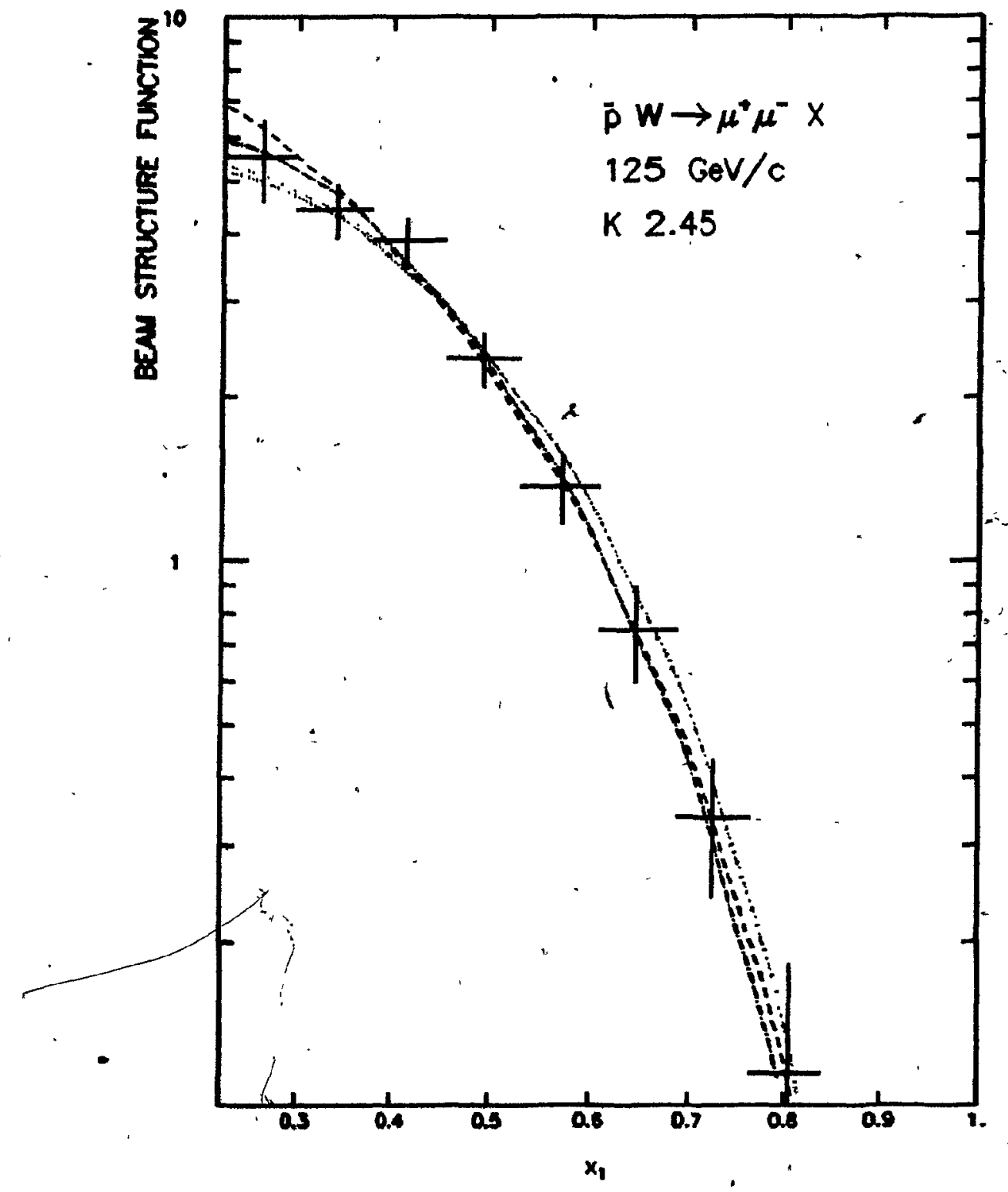

Figure 57 - Antiproton Structure Function Eits

The dot-dashed line shows the antiproton structure function fit, set 5 of Table 13, with a fixed to 0.5. The dashed line shows the curve corresponding to set 6. The dot-blank line and the dotted line show the value of the deep inelastic struoture functions[113] with $Q^{2}=M^{2}$ and, $Q^{2}=25\left(\mathrm{GeV} / \mathrm{c}^{2}\right)^{2}$ respectively. 

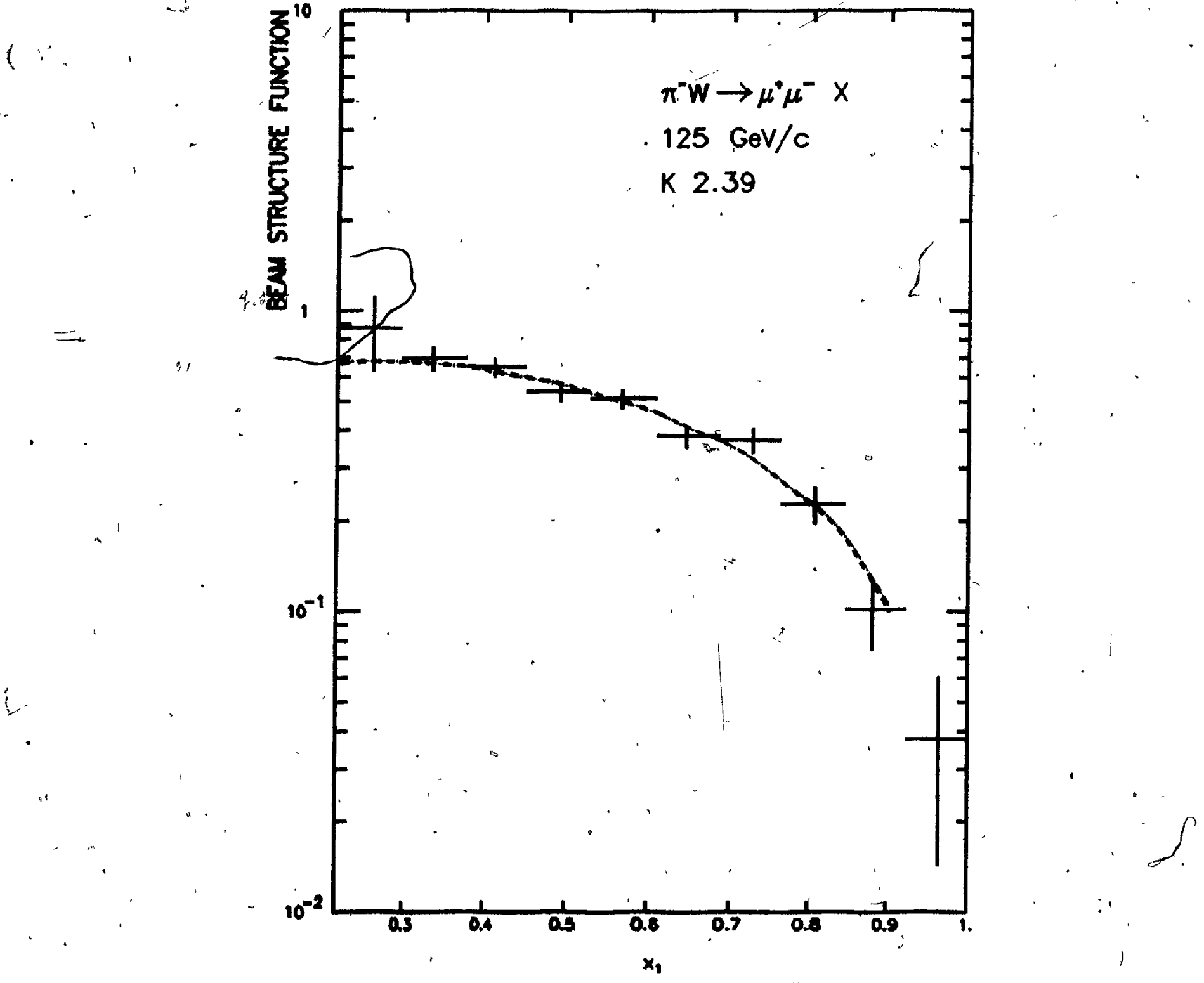

Figure 58 - Pion Structure Function Fits

The dashed line shows the pion valence quark structure function fit using the parameters of set 9 of Table 13, with $\alpha$ fixed to 0.5 . The dot-dashed line shows the curve corresponding to set 10 . Both curves have been multiplied by their respective $K$ factors. 
7.5. Structure Function-Projections

\section{4}

Because the expressions for the cross sections can be approximated by the product of a function of $x_{1}$ and a function of $x_{2}$, it is possible to project average beam and target structure functions from the data. These projections can be used to compare the structure function fits obtained above with the data, and to compare the data to other experiments. :

The cross. section can be written exactly as

$$
\begin{aligned}
& \frac{d^{2} a}{d x_{1} d x_{2}}=\frac{\sigma_{Q}}{x_{1}^{2} x_{2}^{2}}\left[F_{0}\left(x_{1}, Q^{2}\right) G_{0}\left(x_{2}, Q^{2}\right)+G_{1}\right. \\
& \left.F_{1}\left(x_{1}, Q^{2}\right) G_{1}\left(x_{2}, Q^{2}\right)+F_{2}\left(x_{1}, Q^{2}\right) G_{2}\left(x_{2}, Q^{2}\right)\right] .
\end{aligned}
$$

where

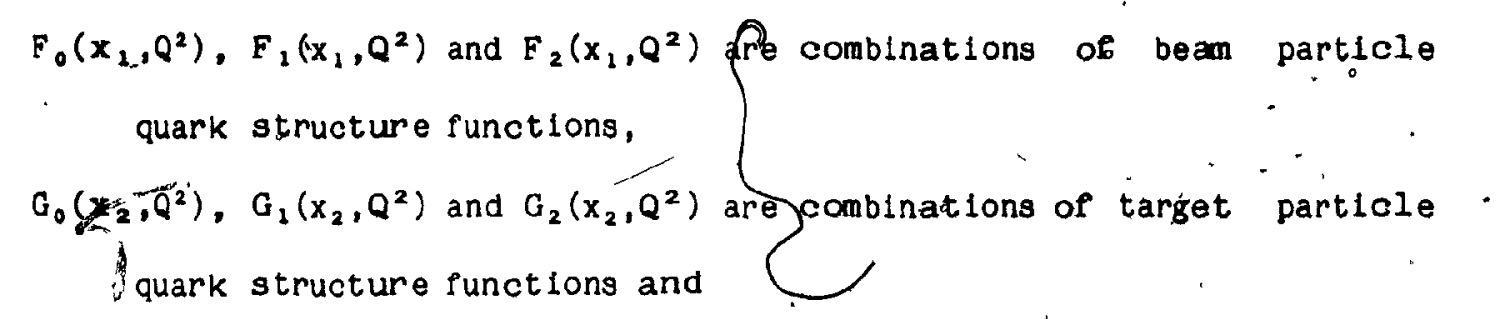

$\rho_{0}=\frac{4 \pi a^{2}}{81 \mathrm{~s}}$ is a normalization factor independent of $x_{2}$ and $x_{2}$ :

For antiproton-tungsten collisions, the structure functions usually chosen as

$$
\begin{aligned}
& F_{0}\left(\dot{x}_{1}\right)=4 u^{p}\left(x_{1}\right)+d^{p}\left(x_{1}\right), \\
& F_{1}\left(x_{1}\right)=d^{p}\left(x_{1}\right), \\
& F_{2}\left(x_{1}\right)=S^{p}\left(x_{1}\right),
\end{aligned}
$$

and

$$
\begin{aligned}
& G_{0}\left(x_{2}\right)=\frac{Z}{A} u^{p}\left(x_{2}\right)+\left(1-\frac{Z}{A}\right) d^{p}\left(x_{2}\right)+s^{p}\left(x_{2}\right) \\
& G_{1}\left(x_{2}\right)=\left(1-\frac{2}{h}\right)\left(u^{p}\left(x_{2}\right)-d^{p}\left(x_{2}\right)\right) \\
& G_{2}\left(x_{2}\right)=\left(1-3 \frac{Z}{\pi}\right) u^{p}\left(x_{2}\right)+\left(4-3 \frac{Z}{\pi}\right) d^{p}\left(x_{2}\right)+10.5 s^{p}\left(x_{2}\right),
\end{aligned}
$$


where

$u^{p}(x), d^{p}(x)$, and $s^{p}(x)$ are the up quark, down quark and sea quark

- structure functions respectively for the proton, and

$\frac{Z}{A}$ is the fraction of protons in the nuclear target.

For pion-tungsten coklisions $F$ and $G$ are given by

$$
\begin{aligned}
& F_{0}\left(x_{1}\right)=V^{\pi}\left(x_{2}\right), \\
& F_{1}\left(x_{1}\right)=S^{\pi}\left(x_{1}\right),
\end{aligned}
$$

and

$$
\begin{aligned}
& G_{0}\left(x_{2}\right)=4 \frac{Z}{A} d^{p}\left(x_{2}\right)+4\left(1-\frac{Z}{A}\right) d^{p}\left(x_{2}\right)+5 S^{p}\left(x_{2}\right) ; \\
& G_{1}\left(x_{2}\right)=\left(1+3 \frac{Z}{A}\right) u^{p}\left(x_{2}\right)+\left(4-3 \frac{Z}{A}\right) d^{p}\left(x_{2}\right)+11 S^{p}\left(x_{2}\right),
\end{aligned}
$$

where $V^{\pi}(x)$ and $S^{\pi}(x)$, are the valence and sea structure functions of the pion and $F_{2}\left(x_{2}\right)$ and ${ }^{\circ} G_{2}\left(x_{2}\right)$ are both zero. The terms have been chosen in such a way that the second and third terms are small compared to the first. If we ignore them, we can write the cross section as

$$
\frac{d^{2} 0}{d x_{1} d x_{2}}=\frac{O_{0}}{x_{1}^{2} x_{2}^{2}} F_{0}\left(x_{1}, Q^{2}\right) G_{0}\left(x_{2}, Q^{2}\right) \text {. }
$$

When this is integrated over $x_{2}$ we obtain

$$
\frac{d 0}{d x_{1}}=\frac{a_{0}}{x_{1}^{2}} \int F_{0}\left(x_{1}, Q^{2}\right) G_{0}\left(x_{2}, Q^{2}\right) \frac{d x_{2}}{x_{2}^{2}}
$$

which we can write as

$$
\frac{d 0}{d x_{1}}=\frac{O_{0}}{x_{1}^{2}} \bar{F}_{0}\left(x_{1}\right) \int G_{0}\left(x_{2}, Q^{2}\right) \frac{d x_{2}}{x_{2}^{2}}
$$

where we have defined, $\bar{F}_{0}\left(x_{1}\right)$, a structure function averaged over $x_{2}$, and thus over $Q^{2}$, as

$$
\bar{F}_{0}\left(x_{1}\right)=\int F_{0}\left(x_{1}, Q^{2}\right) G_{0}\left(x_{2}, Q^{2}\right) \frac{d x_{2}}{x_{2}^{2}},\left[\int G_{0}\left(x_{2}, Q^{2}\right) \frac{d x_{2}}{x_{2}^{2}}\right]^{-1} .
$$

- We can therefore project out the averaged beam structure function, $\bar{F}_{0}\left(x_{1}\right)$, by histogramming the data in terms of $x_{1}$ with a weight given by:

$$
w\left(x_{1}\right)=\frac{x_{1}^{2}}{\sigma_{0}}\left[\int G_{0}\left(x_{2} ; Q^{2}\right) \frac{d x_{2}}{x_{2}^{2}}\right]^{-1} \text {. }
$$

Similarly the averaged target stmucture function, $\bar{G}_{0}\left(x_{2}\right)$, can be projected out by histogramming the events in terms of $x_{2}$ with a weight of

$$
\text { - } w\left(x_{2}\right)=\frac{x_{2}^{2}}{\sigma_{0}}\left[\int F_{0}\left(x_{1}, Q^{2}\right) \frac{d x_{1}}{x_{1}^{2}}\right]^{-1} \text {. }
$$


When the exact expression for the cross section is used, a structure function projection can still be defined as

$$
\bar{F}\left(x_{1}\right)=\frac{x_{1}^{2}}{\sigma_{0}} \frac{d 0}{d x_{1}}\left[\int g_{0}(x / 2) \frac{d x_{2}}{x_{2}^{2}}\right]^{-1}
$$

and corrections can be applied to this quantity to obtain the averaged beam valence structure function $\bar{F}_{0}\left(x_{1}\right)$ : The projection, $\bar{F}\left(x_{1}\right)$, of the data using the deep inelastic scattering structure functions[113] for the target nucteon is shown in Figure 59. The upper curve shows the value for the projection, $\bar{F}\left(x_{1}\right)$, expected from the Drell-Yan model. The lower curve shows the averaged sum of valence quark distributions, $\bar{F}_{0}\left(x_{1}\right)$, that would be

- expected if the cross section could be written exactly as a the product of a function of $x_{1}$ and a function of $x_{2}$. Both curves have been multiplied by the antiproton $K$ factor discussed above. It is clear by comparing the curves that the terms neglected by the projection are small even at the lowest accessible values of $x_{1}$. The profection of the target structure function,

$$
\bar{G}\left(x_{2}\right)=\frac{x_{2}^{2}}{\sigma_{0}} \frac{d a}{d x_{2}}\left[\int F_{0}\left(x_{1}\right), \frac{d x_{1}}{x_{1}^{2}}\right]^{-1},
$$

using the deep inelastic scatttering structure functions for the antiproton is shown in Figure 60. Again the upper curve shows the Drell-Yan prediction for the projection, $\bar{G}\left(x_{2}\right)$. while the lower curve represents the corresponding sum of quark structure functions $\bar{G}_{0}\left(x_{2}\right)$ that would be obtained if the cross section factorized exactly. It is again evident from the Curves that the neglected terms are small.

Figure 61 shows the projection of the antiproton, valence structure function data compared with $150 \mathrm{GeV} / \mathrm{c}$ data from the NA3 experiment uṣing both antiproton and proton data to subtract away non-valence cơntributions. Corrections for the neglected terms have been applied to our data points on this plot. There is good agreement between the experiments about both the shape and normalization of the data points. 


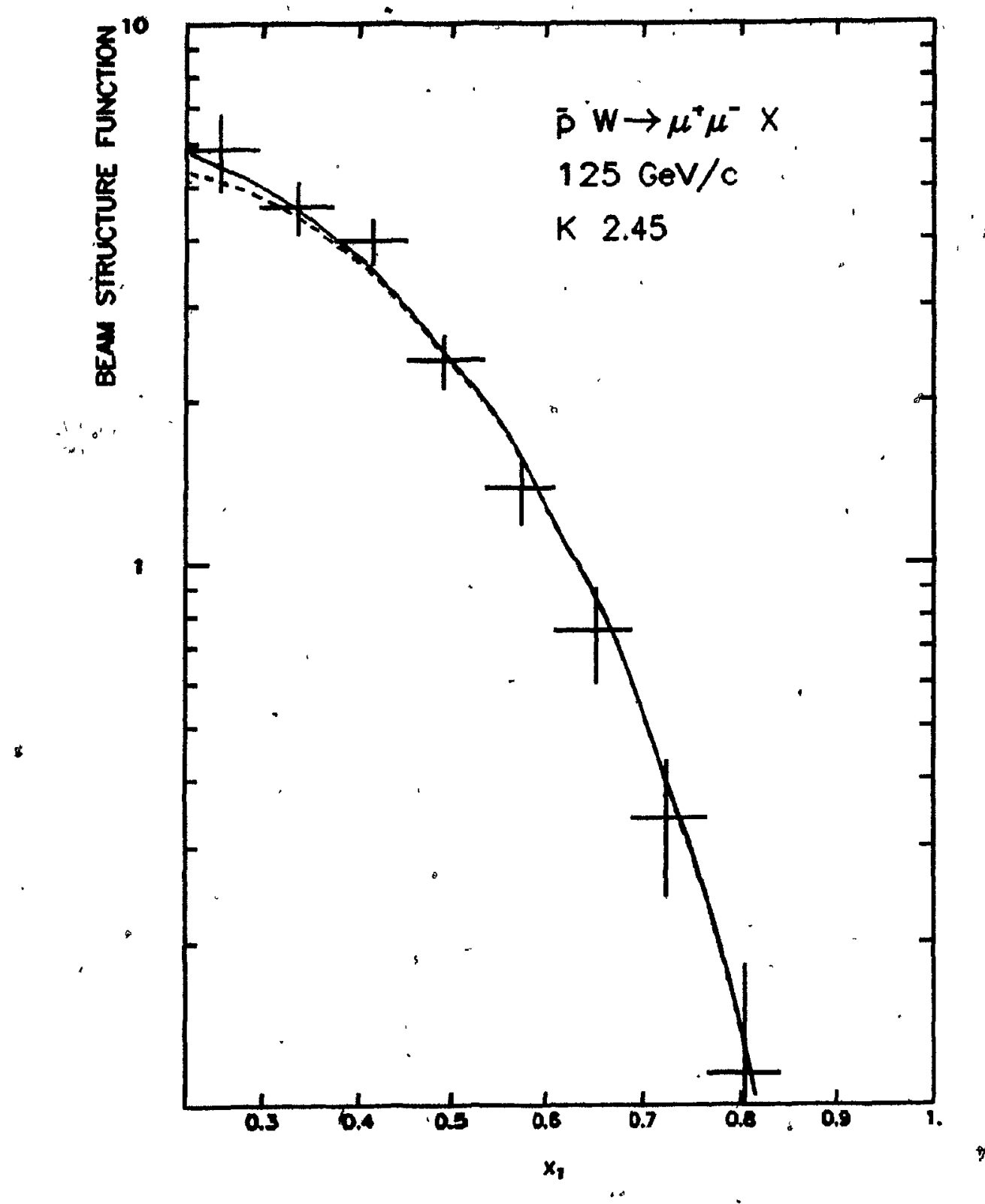

Figure 59 - Antiproton Beam Structure Function Projection

The points show the projection of our antiproton produced data on the $x_{1}$ axis. The upper curve shows the projection, $\bar{F}\left(x_{1}\right)$, expected from the Drell-Yan model. The lower curve shows the averaged sum of valence quark structure functions, $\bar{F}_{0}\left(x_{1}\right)$, that would be expected if the cross section could be written exactiy as the product of a function of $x_{1}$ times a function of $x_{2}$. 


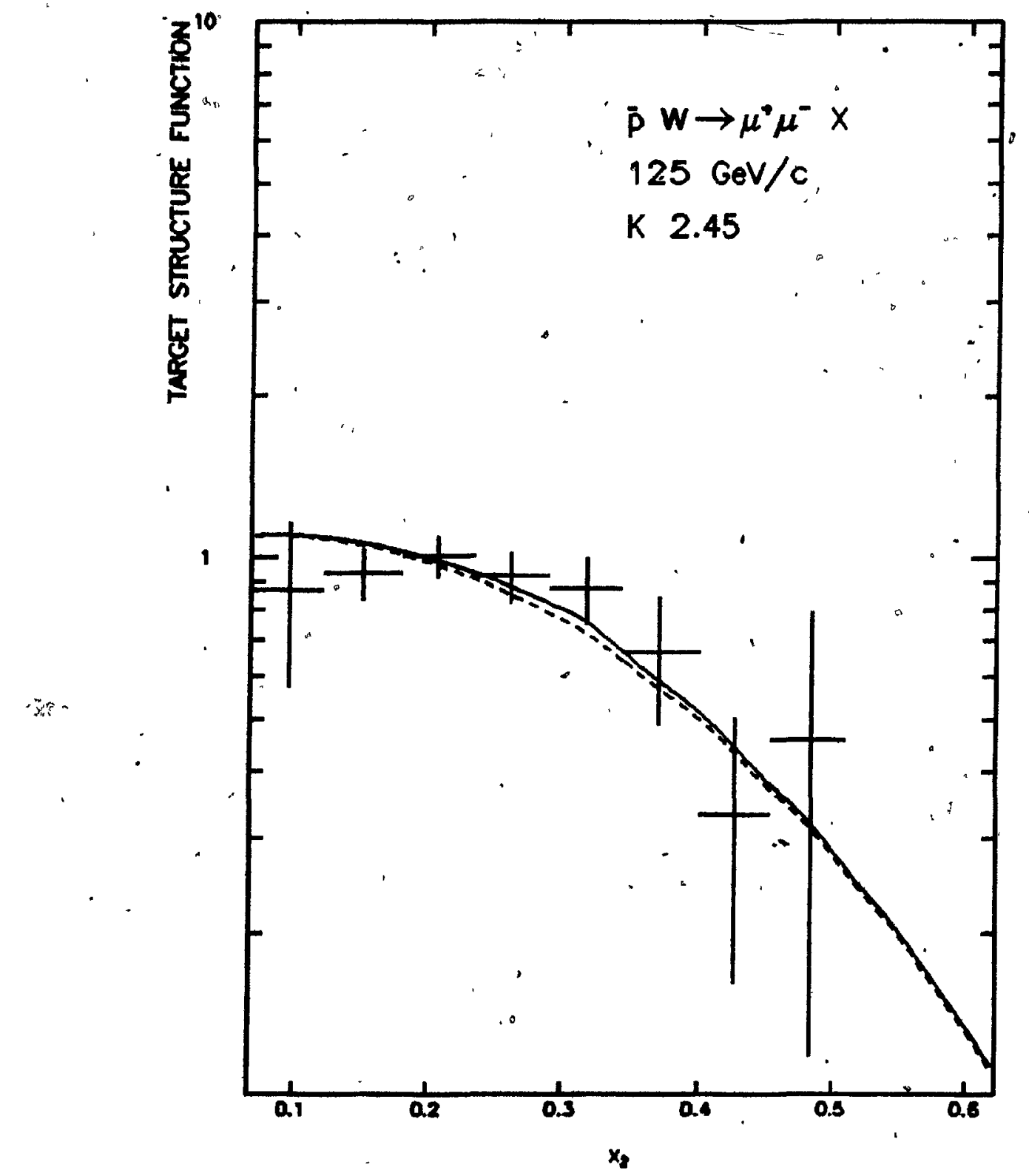

Figure 60 - Antiproton Target Structure Function Projection

The points show the projection of our antiproton produced data on the $x_{2}$ axis. The upper curve shows the result, $\bar{G}\left(x_{1}\right)$, expected from the Drell-Yan model. The lower curve shows the valence quark structure function $\bar{G}_{0}\left(x_{1}\right)$ 


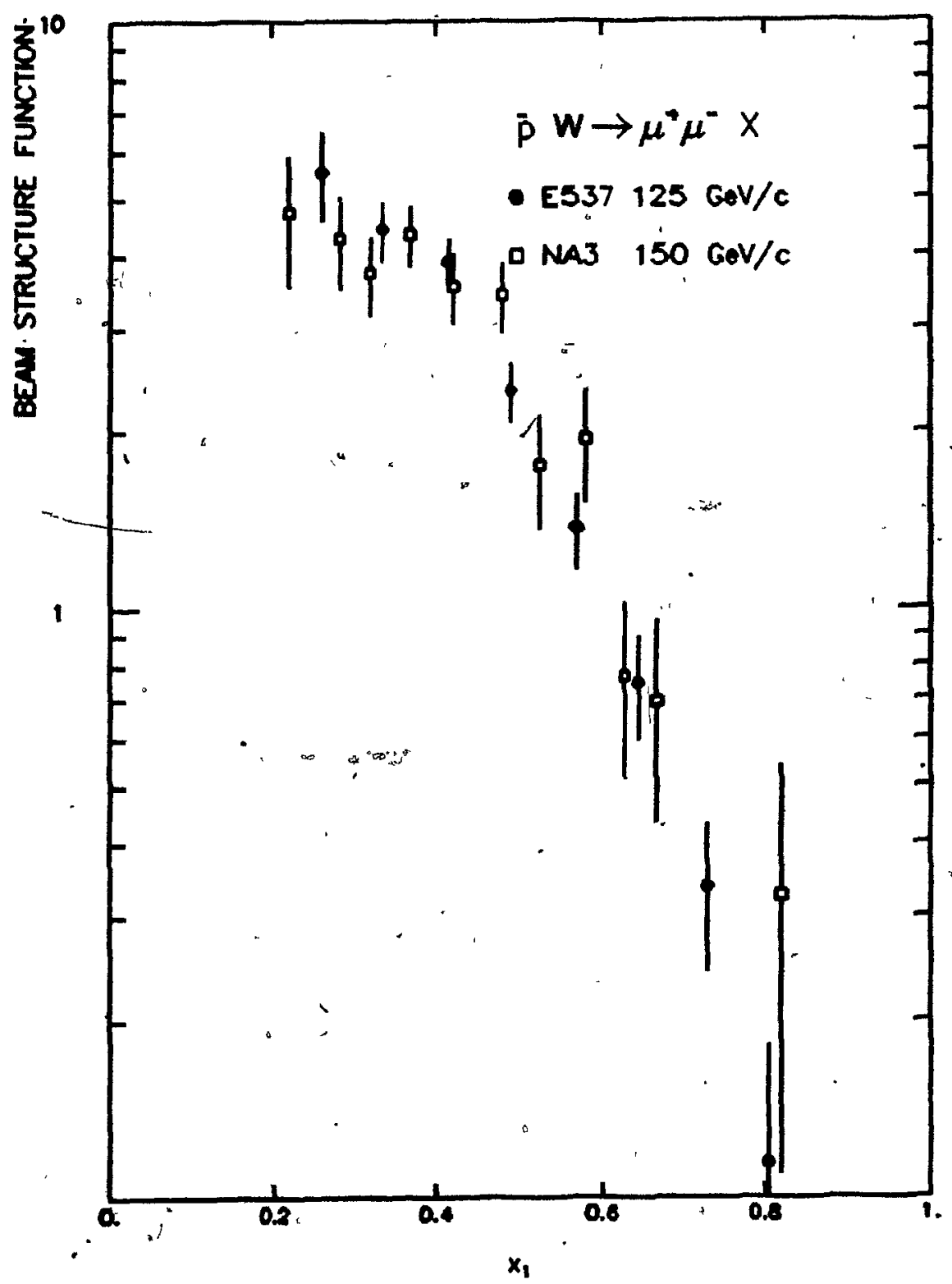

D

Figure 61 - Antiprotion Beam Structure function Compared To NA3

Our projected antiproton valence quark structure function is compared with data obtained by the NA3[10] collaboration. 
Figure 62 shows the beam structure function projection, $\bar{F}\left(x_{1}\right)$; projected from the pion data using the deep inelastlc structure functions for the target nucleon. The upper curve shows the results expected for the projection, $\vec{F}\left(x_{1}\right)$, from the Drell-Yan model. The lower curve shows the sum of valence quark distributions, $\bar{F}_{0}\left(x_{2}\right)$, that would be expected if the cross section factorized exactly. Here we have used the parameters from'set 9 of Table 13 for the pion valence structure function, the NA3[46] $200 \mathrm{GeV} / \mathrm{c}$ results for the pion sea, and the deep, inelastic scattering structure functions[113] for the target nucleon, to calculate the Drell-Yan curves. The Drell-Yan curves have been multiplifed by a factor of

$$
K_{\pi}-=2.39
$$

to normalize to the measured cross setion. The projection of the farget. structure function, $\bar{G}\left(x_{2}\right)$, for the pion data is shown in Figure 63 . The : curves again show the values expected for $\bar{G}\left(x_{2}\right)$ and $\bar{G}_{0}\left(x_{2}\right)$ from the Drell-Yan model, and agaln the terms neglected by the projection are 'small. Comparisons of our beam structure function with data from the NA3[46], CIP[40], Omega[43], and GOLIATH[44] collaborations.are shown in Figure 64. Note that we have adjusted the CIP points on this plot to reflect our assumed A dependence. The agreement, between ourselves and the other experiments is very good over the entire $x_{1}$ range, both in shape and absolute value. Our target structure function is compared to the results. obtained by NA3[46] and CIP[40] in Figure 65. Again the agreement is quite good in both cases.

\subsection{Conclusion}

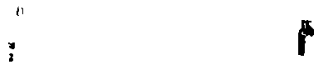

This thesis has reported the results of an experlment whlch has measured muon pair production in $125 \mathrm{GeV} / \mathrm{c}$ antiproton-tungsten and 


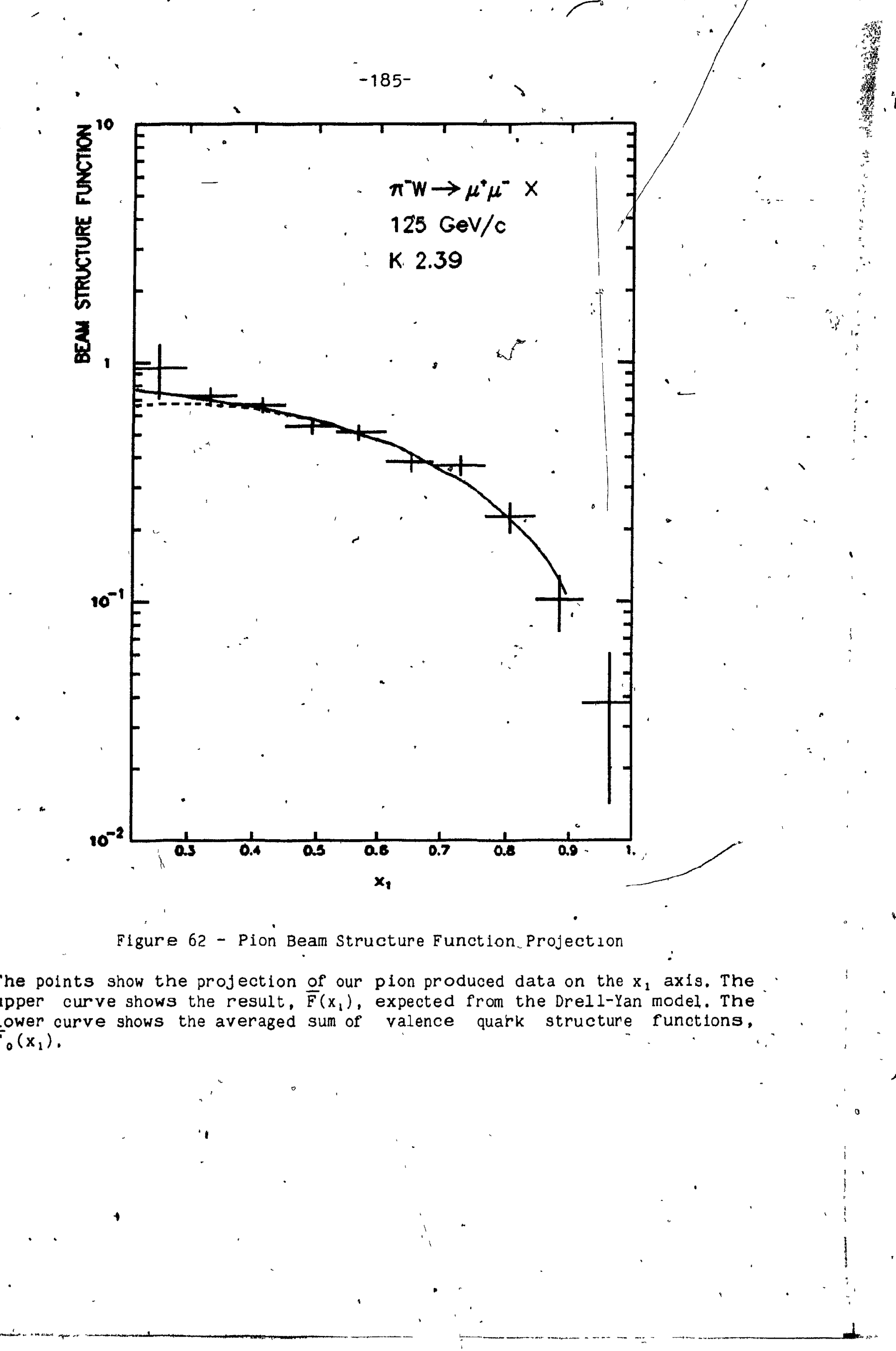




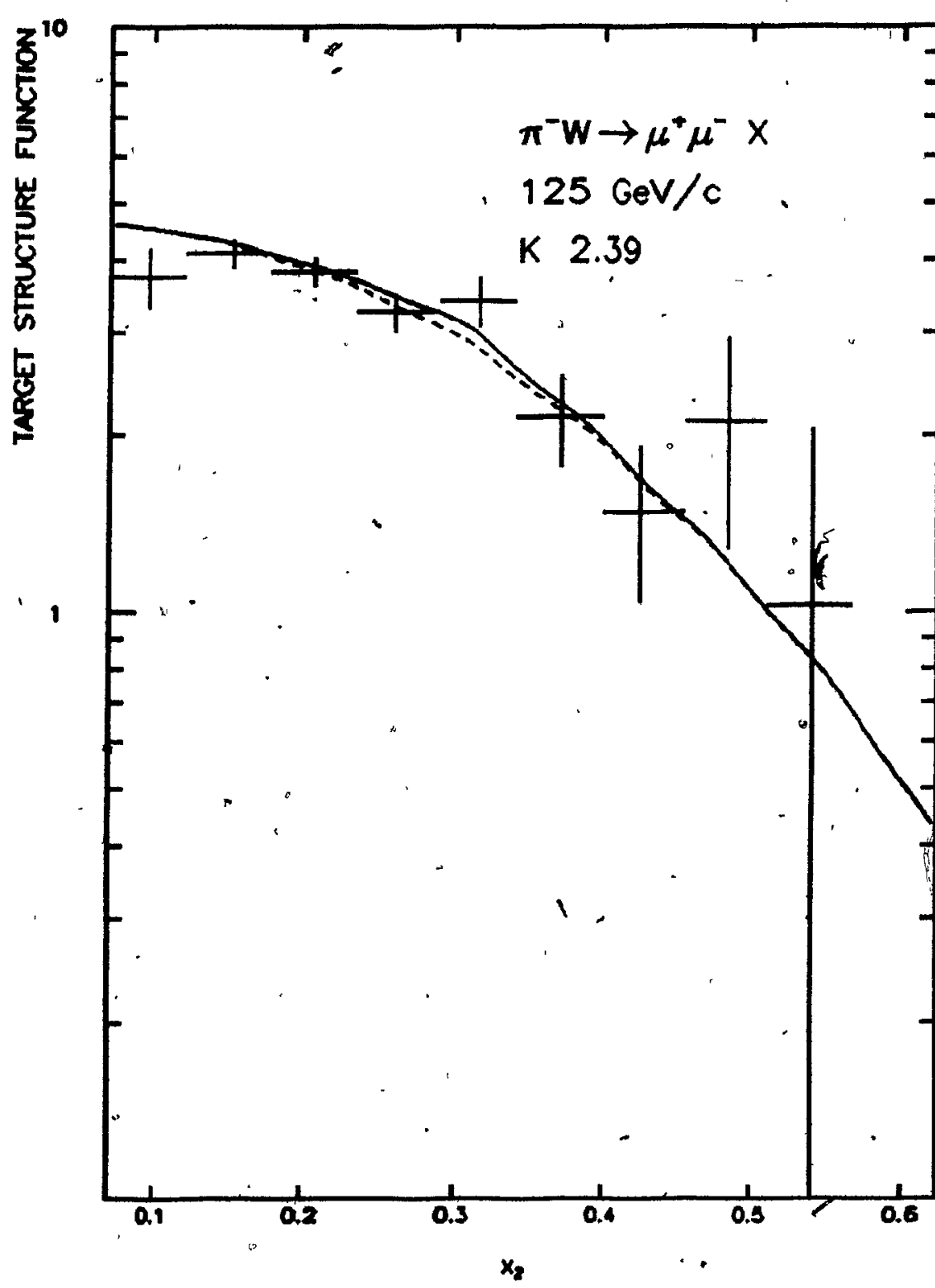

Figure 63 - Pion Target Structure Function Projection

The points show the projection of our pion produced data on the $x_{2}$ axis. The upper curve shows the result, $\bar{G}\left(x_{2}\right)$, expected from the Drell-Yan model. The lower curve shows the valence quark structure function $\vec{G}_{0}\left(x_{2}\right)$. 


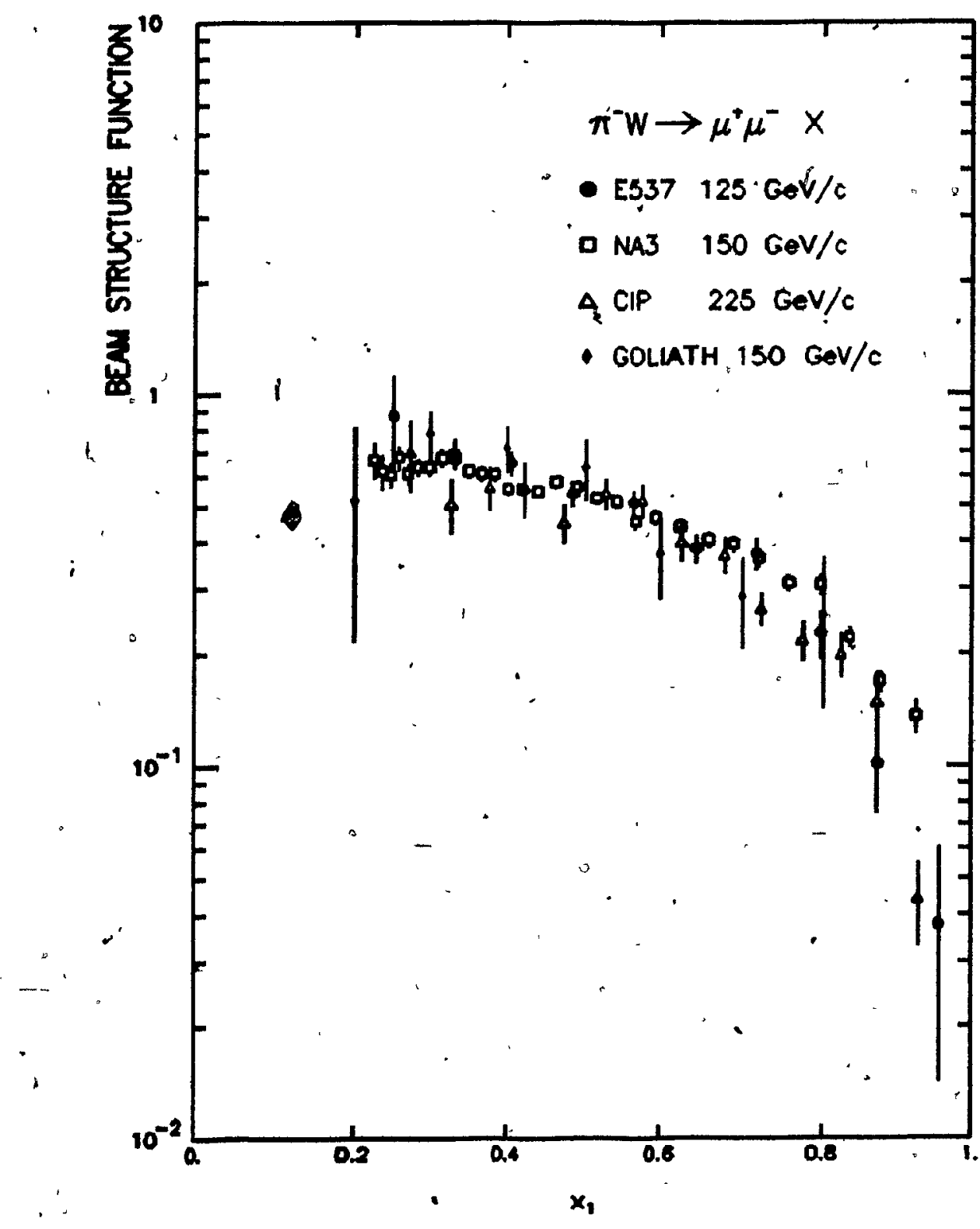

Figure 64 - Pion Beam, Structure Function Comparison

Our projected pion valence quark structure function is compared with data from the NA3[46], CIP[40], and Goliath[44] experiments. There is good agreement between the four experiments. 
plon-tungsten collisions using a tungsten target, a, spectrometer with good acceptance for masses between 4.0 and $9.0 \mathrm{GeV} / \mathrm{c}^{2}$ with $\mathrm{x}_{\mathrm{F}} \geq 0$, and a beam tagging system capable of counting and identifying individual beam particles. The measurement of the cross section for muon pair production by antiprotons provides an unambiguous test of the Drell-Yan model and QCD corrections since the valence quark structure functions of the nucleon have been accurately determined in deep inelastic lepton scattering experiments. Our'sample 387 events and our identification of individual beam particles give us the best measurement of the cross section for muon pair iproduction by antiprotons to date. Because of the scaling properties of the cross section predicted by the Drell-Yan model, comparison of the pion prpduced data to results from other experiments using different beam energies provides a valuable cross-check on the antiproton results as well as testing the model itself.

We find that both the kinematic dependences and the absolute normalization of the cross section for pion produced pairs agrees well with measurements by other experiments. We also find good agreement with the only other data for antiproton" produced pairs. We" have compared our measurements of the differential cross sections, $\frac{\mathrm{do}}{\mathrm{dM}}$ and $\frac{\mathrm{do}}{\mathrm{dx}_{\mathrm{F}}}$, for the production of muon pairs in antiproton-tungsten collisions to the predictions of the Drell-Yan model using nucleon structure functions measured' In deep inelastic scattering and find good agreement between the data and the model if we multiply the predictions by.

$$
K_{\bar{p}}=2.45
$$

The leading order QCD corrections make only small changes in the predicted kinematic dependences of the cross sections but increase the absolute magnitude of the predicted cross section by a factor of 1.87 , so that we 
find that the experimental cross section exceeds the leading order prediction by a factor of

to

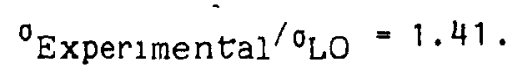

Calculations of the cross section for mubn pair production in pion-tungsten collisions involve large uncertainties because the pion valence quark structure function cannot be measured in inelastic scattering experiments. The pion produced, data does, however, exhibit the qualitative features expected from the model. The differential cross section, do, falls more - quackly with increasing mass for, antiprotons than for pions as would be expected.from the model on the basis of counting rule arguments. The differential cross section, $\frac{d_{0}}{d x_{F}}$, also falls more quickly with increasing $x_{F}$ for antiprotons as would be expected from the same arguments. The angular distributions, $\frac{1}{0} \cdot \frac{d \sigma}{d \cos \theta}$ and $\frac{1}{0} \cdot \frac{d \sigma}{d \phi}$, are also consistent with the expectations.of the Drell-Yan model for both the plon produced data and, the antiproton produced data.

The Drell-Yan formula has been inverted and used to obtain the antiproton structure function from the data: Again we find good agreement between our results and data obtained by the NA3 collaboration[10] using a $150 \mathrm{GeV} / \mathrm{c}$ beam. The shape of the antiproton structure function is also in good agreement with the shape of the proton structure function measured by . deep inelastic scattering experiments. The plon structure function extracted in the same way agrees well with measurements by other muon pair experiments both in shape and magni tude.

It is clear that antiproton experiments, with higher statistics are necessary to make detailed comparisons of the shapes of the kinematic distributions with the DrelI-Yan model and the first order QCD predictions 
Appendix I - E537 Collaboration

E. Anassontzis, S. Katsavenas, P. Kostarakis, C. Kourkoumelis, A. Markou, L.K. Resvanis G. Voulgaris University of Athens, Athens, Greece

M. Binkley, B. Cox, J. Enazonio, C. Hojvat, D. Judd, R.D. Kephart, P.K. Malhotra, P.O. Mazur, C.T. Murphy, F. Turkot, R.L. Wagner, D. Wagoner, W. Yang

Fermi National Accelerator Laboratory, Batavia, IL 6051,0, USA

H. Aret1, S. Conetti, P. Lebrun, D. Ryan, T. Ryan, W. Schappert, D. Stairs

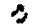
McGill University, Montreal, Province of Quebec, H3A2T8, Canada

C. Akerlof, X, Cui, P. Kraushaar, D. Nitz, R. Thun, L. Wang University of Michigan, Arbor, MN 48109, USA

He Mao, Zhang Nai-jian

Shandong University, Jinan, Shandong, Peoples Republic of China 


\section{Appendix II - Vertex Reconstruction}

\section{$\$$}

The resolution of the spectrometer was limited by multiple soattering of the muons in the, copper hadron filter. Substantial improvement was achleved in the mass resolution by fixing the production vertex at the centre of the target as discussed in the text. This was possible once the event was known to have originated in the target, but the misassignment of an event produced in the dump to the target could result in a substantial error in the reconstructed mass. A simple distance of closest approach method allowed most events to be unambiguously assigned to ei ther the dump or the target but enough events remained unresolved to cause concern about bias in the kinematic distributions. A better determination of the production point was made by finding the most probable vertex for the palr of muons using a Gaussian model for multiple scattering,<smiles>C1=C2CC3C1C3C2</smiles>

The model for multiple scattering used here is due to Fèrmi and is discussed by Rossi and Greisen[131] and in Rossi's book[132]. The algorithm used to find the best vertex is a straightforward adaptation of techniques originally applied to cosmic ray tracks in cloud chambers[133], and was more . recently used in other muon pair experiments[128][134]. The implementation of the algorithm in this case was discussed in some detal.l by Kraushaar[85]. but will be outlined here because of Its importance?

In the limit of small scattering angles, negligible energy loss, many. separate scatters, and working in the projection on the Y-Z plane as illustrated in Figure 66, the probability that a particle travelling along the $Z$ axis and entering a scatterer at $Y=Z=0$, w111 emerge a distance $Y$ from the $Z$ axis, with an angle. $\theta_{Y}$ with respect to the $Z$ axis, is given by . 


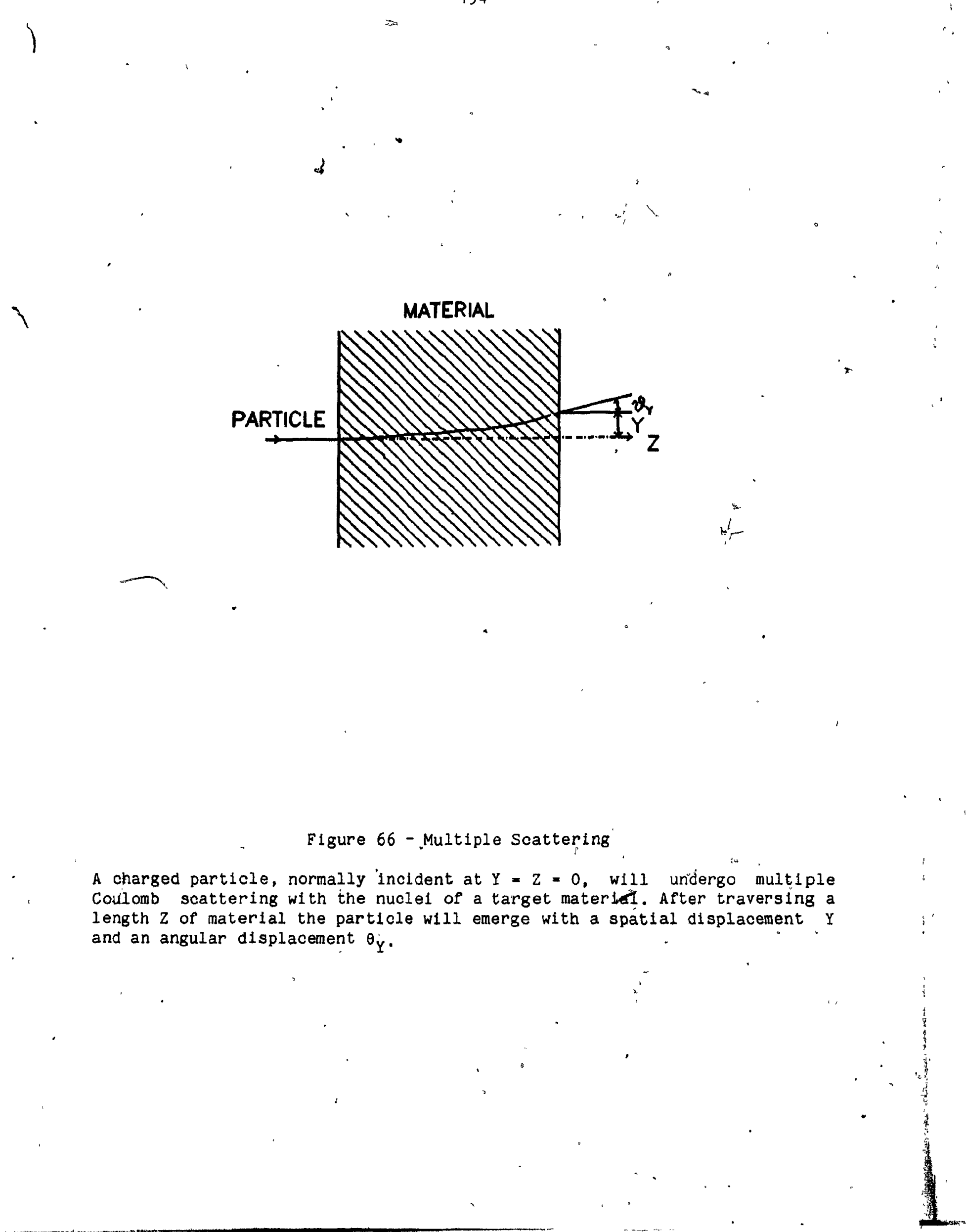


$\int_{1} P\left(Y, \theta_{Y} \mid Z\right)=\frac{2 \sqrt{3}}{\pi \theta_{Y R M S}^{2} Z^{2}} \exp \left(-\frac{4}{\theta_{Y R M S}^{2}}\left(\theta_{Y}-3 \theta_{Y} \Psi+3 \psi^{2}\right)\right)$

where $\psi$ is the dimensionless ratio

$\psi=Y / Z$.

The plane projected RMS scattering angle is commonly taken to be[13]

where

$$
\theta_{\mathrm{YRMS}}=\frac{0.015}{\beta \mathrm{p}} \sqrt{\mathrm{Z}_{\mathrm{rad}}}
$$

$\beta$ is the velocity of the particle in units of $c$,

$\mathrm{p}$ is the momentum of the track in $\mathrm{GeV} / \mathrm{c}$,

$Z$ is the length of the scatterer, and

$\mathrm{Z}_{\mathrm{rad}}$ is the of the radiation length[13] for the scattering material.

The probability that a particle which enters sthe scatterer at $Y=Z=0$, travels to an intermediate point $Y_{1}, Z_{1}$ with angle $\theta_{1}$, then emerges at a point $Y_{2}, Z_{2}$, at an angle $\theta_{2}$, is given. by

$$
\begin{gathered}
P\left(Y_{1}, Y_{2}, \theta_{1}, \theta_{2} \mid Z_{1}, Z_{2}\right)= \\
P\left(Y_{11}^{\prime}, \theta_{1} \mid Z_{1}\right) \cdot P\left(Y_{2}-Y_{1}-\theta_{1}\left(Z_{2}-Z_{1}\right), \dot{\theta}_{2}-\theta_{1} \mid Z_{2}-Z_{1}\right)= \\
A \cdot \exp \left(-X^{2} / 2\right)
\end{gathered}
$$

where we have defined the various parameters that appear as follows:

$$
\begin{aligned}
& A=2 \sqrt{3} /\left[\pi \theta_{1}{ }^{2} R_{M S} K^{2}(1-k)^{2} z_{2}{ }^{4}\right] \text {, } \\
& \ldots x^{2 / 2}=\frac{4}{\theta_{\mathrm{YRMS}}^{2} Z_{2}}\left(\delta^{2}+\xi^{2}+\omega^{2}+\theta^{2}\right), \\
& \delta^{2}=\frac{i}{k(1-k)}\left(\theta_{2}+\frac{3}{2} \frac{2 k-1}{1-k} \psi_{1}-\frac{3}{2} \frac{k}{1-k} \psi_{2}+\frac{k}{2} \theta_{2}\right)^{2}, \\
& \xi^{2}=\frac{3}{4} \frac{1}{k(1-k)^{3}}\left(\psi_{2}-k(3-2 k) \psi_{2}+k(1-k) \theta_{2}\right)^{2} \text {, } \\
& \omega^{2}=3\left(\psi_{2}-\frac{1}{2} \theta_{2}\right)^{2} \text {, }
\end{aligned}
$$


and the dimensionless variables are

$$
k=Z_{1} / Z_{2}
$$

$\psi_{1}=Y_{1} / k Z_{2}$, and

$\Psi_{2}=Y_{2} / Z_{2}$

Integration over all values of $\theta_{1}$ using

$$
d \theta_{1}=\sqrt{k(1-k)} d \delta
$$

gives the probability that a particle entering a scatterer at $Y=Z=0$ is observed at $Y_{1}, Z_{1}$ and emerges from the scatterer at $Y_{2}, Z_{2}$ with angle $\theta_{2}$.

It is straightforward to generalize to the case of a particle produced at a point $Y_{0}, Z_{0}$, at an angle $\theta_{0,}$ being observed at a point $Y_{1}, Z_{1}$ and emerging at a point $Y_{2}, Z_{2}$ with angle $\theta_{2}$ by making the following substitutions:

$$
\begin{aligned}
& \theta_{2} \rightarrow \theta_{2}-\theta_{0}, \\
& Y_{2} \rightarrow Y_{2}-\left(Z_{2}-Z_{0}\right) \theta_{0}, \text { and } \\
& Y_{1} \rightarrow Y_{1}-\left(Z_{1}-Z_{0}\right) \theta_{0} .
\end{aligned}
$$

For the case of two particles produced at a common vertex at $Y_{0}, Z_{0}$ with. angles $\theta_{01}$ and $\theta_{02}$, and being observed with $Y_{11}, Y_{21}, \theta_{21}$ and $Y_{12}, Y_{22}, \theta_{22}$ respectively, as 'shown schematicalfy' in Figure 67, the total probability will be the product of the individual probabilites. Scattering in the $x$ direction can be treated in exactly the same fashion.

Here the drift chambers were used to give measurements of the track , positions and slopes downstream of the dump, that is at $z_{2}$. The absorber: chamber gave information about the track coordinates $2 / 3$ of the way through 


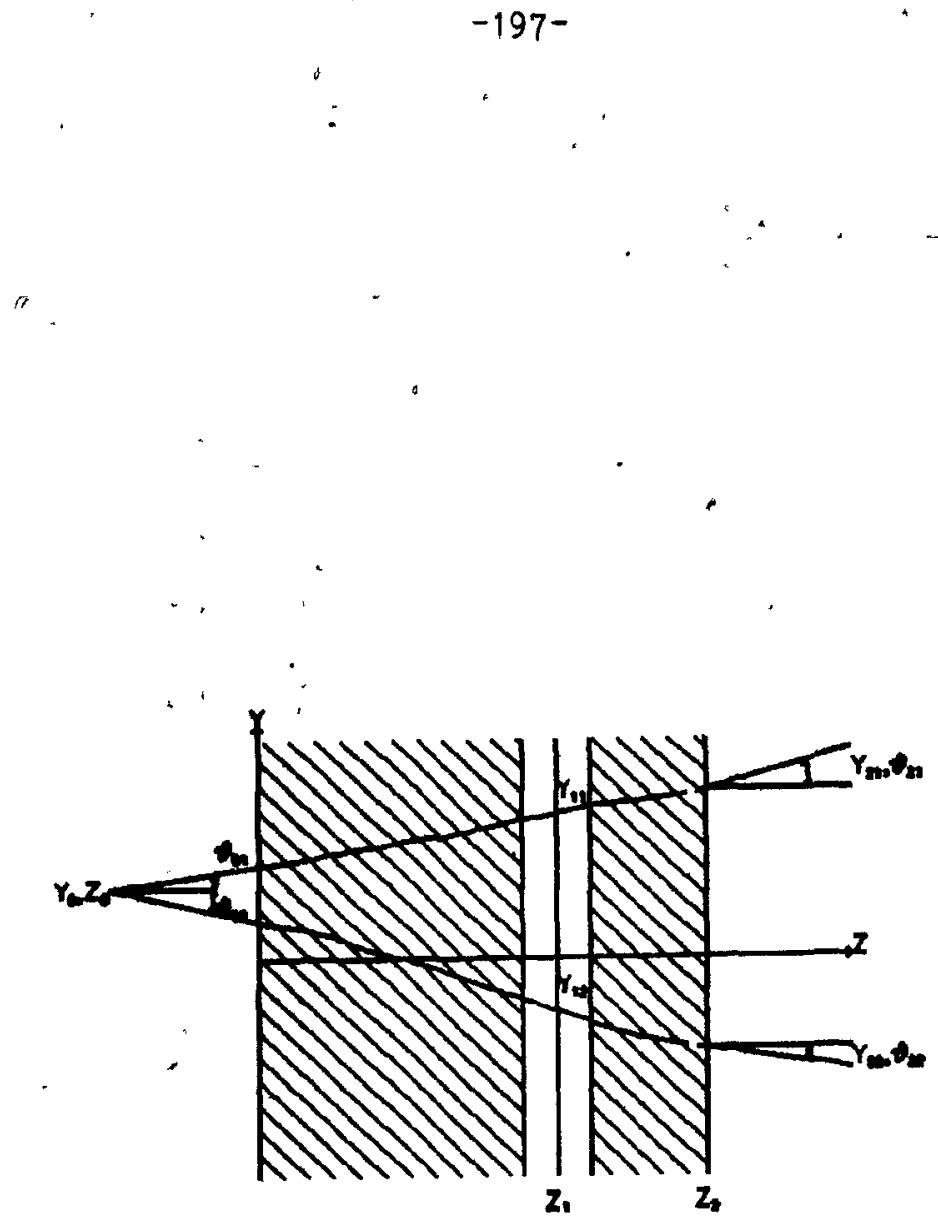


the dump at $Z_{1}$. The beam chambers were used to determine the $X$ and $Y$ of the production vertex. The $z$ position of the production vertex was found by numerically searching for the values of $z_{0}$ and the production angles, $\theta_{0} 1$ and $\theta_{02}$, that gave the the highest value of the probability function in the above expressions. The search was performed using ZXSSQ, a FORTRAN callable nonlinear minimization routine from the IMSL library[135]. 
- The incident beam is attenuated in the target according to the well known exponential law,

\section{$I(Z)=I_{0} \exp \left(-Z / L_{A b s}\right)$,}

where $L_{A b s}$ is the absorption length of the target material. If exach particle removed from the beam creates an secondary particles in the energy range $d E$, then the number of secondaries, di, created in the interval dz will be

$$
\frac{d I}{d z}=-\frac{d I}{d z} \frac{d h}{d E}
$$

If the absorption length does not depend on energy, each sefcondary created in an element of length de will see a target of effective length

$$
L_{E f f}(Z)=L_{A b s}\left[1-\exp \left(-\frac{L-Z}{L_{A b s}}\right)\right]
$$

where $L$ is the physical length of the target. If $o(E)$ denotes the cross section for the production of muon pair's by secondary particles of energy $E$ : - the number of muon pairs dN (within constant factors) produced by secondarles created in the element of length $\mathrm{dz}$ will be

$$
\frac{d N}{d z}=-I_{0} / L_{A b s} \exp \left(-\frac{L}{L_{A b s}}\right) \cdot L_{A b s}\left[1-\exp \left(-\frac{L-Z}{L_{A b s}}\right)\right] \cdot \int_{0}^{E_{\max }}-\frac{d n}{d E} O(E) d E .
$$

Integrating from $Z=0$ to $Z=L$ gives

where we have defined

$$
N(L)=N_{0}\left[1-\left(1+\frac{L}{L_{A b S}}\right) \exp \left(-\frac{L}{L_{A b s}}\right)\right]
$$

$$
N_{0}^{*}=I_{0} L_{A b s} \int_{0}^{E_{\max }} \frac{\mathrm{dn}}{\mathrm{dE}} \sigma(E) \mathrm{dE} .
$$

This quantity depends only on the physical processes taking place and not on the length of the target.

In contrast, the number of muon pairs produced by primary particles depends on the target length as

$$
N_{\text {Direct }}(L)=N_{\text {oD } 1 \text { rect }}\left[1-\exp \left(-\frac{L}{L_{A b s}}\right)\right] \text {, }
$$


where.

$$
-200-
$$

C

$$
\mathrm{N}_{\text {ODidfect }}=I_{0} \cdot 0\left(\mathrm{E}_{\max }\right) \cdot \mathrm{L}_{\mathrm{Abs}} \text {. }
$$

- iss."

which again does not depend on the target length. The measured cross section will then be

$$
\begin{gathered}
0_{\text {Measured }}(L)=\left[N_{\text {Direct }}(L)+N_{\text {Secondary }}(L)\right] /\left[I_{0} L_{A b s}\left(1-\exp \left(-\frac{L}{L_{A b s}}\right)\right)\right] \\
\Rightarrow{ }^{0}{ }_{\text {Direct }}{ }^{+\sigma_{\text {Reinteraction }}}\left[1-\frac{L}{L_{A b s}}\left(\exp \left(\frac{L}{L_{A b s}}\right)-1\right)\right]
\end{gathered}
$$

where

- 'Direct is the cross section that would be measured using an infinitesimally thin target, and

OReinteraction is a constant independent of the length of the target. This expression can be fit to cross sections measured using targets of different lengths to obtain ooprect and ${ }^{O}$ Reinter action. Once these are given, the expression can be used to calculate the correction for. reinteraction for a target of any length. 


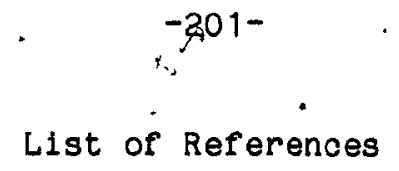

1. Christenson, J., et al., Phys.Kev.Lett. 25, 1523 (1970)..'

2. Rutherford,E., Phil.Mag. 21, 669 (1911).

3. Chadwick, J., Nature 129, 312 (1932).

4. Chadwick, J., Proc.Roy.Soc. A136, 692 (1932).

5. Hofstadter,R., Ann.Rev.Nucl.Sci. 7, 231 (1958).

6. Gell-Mann,M., Phys.Lett. 8,214 (1964).

7. Zweig,G., CERN Report 8182/Th.401, CERN, Geneva, Switzerland $(1964)$.

8. Pennington,M.R., Rep.Prog.Phys. 46, 393 (1983).

9. Marciano,W., and H.Pagels, Phys.Rept. $\underline{36}, 137$ (1978).

10. Badier,j., et al., Phys.Lett. 96B, 422 (1980),

11. Drell,S., and Yan,T.M., Phys, Rev.Lett,.25, 578 (1970).

12. Drell,S., and Yan,T.M., Ann.Phys. 66, 578 (1970).

: 13. Particle Data Group, Review of Particle Properties, Published in ${ }^{\circ}$ Phys.Lett. 111B, 1 (1982).

14. Politzer,H.D., Nucl.Phys. B129, 301 (1977).

15. Bodwin,G., Brodsky,S., and Lepage,G., Phys.Rev.Lett. 47, 1799 (1981).

16. Lindsay,W., Ross, D., and Sachrajda,C., Nucl.Phys. B222, $189,(1983)^{8}$.

17. Lindsay,W., Ross, D., and Sachrajda,C., Nucl.Phys. B214, 61 (1983).

18. Lyons,L., Prog.Part.and.Nucl.Sci. I, 169 (1981):

19. Stroynowski,R., Phys.Rept. 71, 1 (1981).

20. Kenyon, I., Rept.Prog, Phys. 45, 1264 (1982).

21. Tran Than Van, J., ed., 1981 Moriond Workshop, Les Aros; Editions Frontiers, Dreux, France.

22. Berger,E., et al., Proceedings of the Drell-Yan Workshop, Fermilab, Batavia, IL 60510, USA. (October 1982).

23. Gottfreid,K, , and Jackson,J.D.; Nuovo Cimento 33, 309 (1964).

24. Collins, J., and Soper, D., Phys.Rev.D 16, 2219 (1977).

25. Lam,C.S., and Tung,W.K., Phys, .Rev.D 18, 7 (1978). 
26. Collins, J. Phys.Rev.Lett. 42, 291 (1979):

- 27. Politzer,H.D., Nucl.Phys. B129, 301 (1977).

28. Sachradja,C.T., Phys.Lett. 73B, 185 (1978).

29. Kubar,J., et al., Nucl.Phys. B175, 271 (1979).

30. Altarelli,G., et al.; Nucl.Phys. B157, 461 (1979).

31. Curci,G., and M.Greco, Phys.Lett. 92B, 175 (1980).

32. Contogouris,A., and L.Meau, Phys.Rev D 28, 1644 (1983).

33. Marleau,L., Unpublished Ph.D. Thesis, McGill University (1983).

34. Kenyon[20] reviews Drell-Yan A.dependence.

35. Aubert,J., et al., Phys.Lett. 123B, 123 (1983).

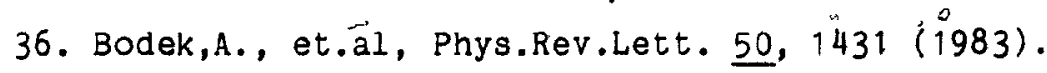

37. Bodek,A., et al., Phys.Rev.Lett. 51, 534 (1983).

38. Ito,A., et al., Phys.Rev.D 23, 604 (1981).

39. Anderson,K., et al., Phys.Rev.Lett. 42,944 (1979).

40. Hogan,G., Phys.Rev.Lett. 42, 948 (1979).

41. Corden,M.J., et al., Phys.Lett. 76B, 226 (1978).

42. Corden,M.J., et al., Phys.Lett. 96B, 411 (1980).

43. Corden,M.J., et al., Phys.Lett. 96B, 417 (1980).

44. Barate,R., et al., Phys.Rev.Lett. 43,1541 (1979).

45. Badier,J., et al., Unpublished Preprint CERN-EP/84-16 CERN, Génèva, Switzerland (February 1984).

46. Badler,J., et al., Phys.Lett. 89B, 145 (1979).

47. Badier,J., et al., Unpublished Preprint, CERN/EP 80-148, CERN, Geneva, Switzerland (August 1980).

48. Lefrancois, L., 1980 Proc.Int.Conf. on High-Energy Physics, Madison. AIP Conf 68 pg. $1319(1980)$.

49. Badier,J., et al., Zeit.Phys.C 11, 195 (1981).

50. Michelini,A., Unpublished Preprint CERN-EP/81-128, CERN, Geneva, Switzerland (1981).

51. Varela,J., Ecole Poly. Paliseau Preprint LPHNE/X 83-02, Presented to 1983 Moriond Conf. La Plagne, (January 1983). 
52. Falciano,S., et al., Unpublished Preprint CERN-EP/81-52, CERN, Geneva, Switzerland (1981).

53. Lederman,L., et al., Fermilab Proposal P605, Fermilab, Batavia, IL 60510 , USA. (1978).

54. Anderson,K., et.al, Fermilab Proposal P615, Fermilab, Batavia, IL 60510, USA. (1978).

55. J.R.Sanford, Ann.Rev.Nucl.SC1. 26, 151 (1976).

56. Cox,B., et àl., Unpublished P-WEST Design Report, Fermilab, . Batavia, IL 60510, USA. (March 1977).

57. Cox,B., Fermilab Report, pg.6 Fermilab, Batavia, IL 60510, USA. (January 1979).

58. Agoritsas, V., AGS Tech. Note 136, BNL, Upton, NY 11973 USA., (June 28 1977), discusses the SEMs used at Fermilab.

59 Hornstra, F. , and Simanton, J., NIM 7T, 303 (1970). .

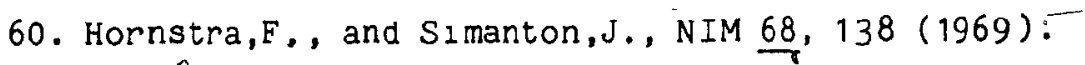

61. Kephart,R., and Kerns,C., Unpublished Technical Memo TM-1157. Fermilab, Batavia, IL 60510, USA. (1979).

62. Mfg. Nuclear Enterprises Corp. : San Carlos, CA 94070 USA.

63. Mfg. Amperex Corp., Elk Grove Village, IL .60007, USA.

64. Mfg. RCA Corp., Des Plaines, IL 60018, USA.

65. Kerns,C:, IEEE Trans.Nucl.Sci. NS-24, 353 (1977).

66. Mfg. Lecroy Research Services, Spring Valley, NY 10977, USA.

67. US AEC Committee, Standard Nuclear Instrument Modules, TID-20893 (Rev. 4), GPO, Washington, DC, USA. (1974).

68. Mfg. Pilot Chemicals Division, New England Nuclear Corp., Waterton, MS 02172, USA.

69. Charpak,G., Ann.Rev.Nucl.Sei. 20, 195 (1970).

70. Rice-Evans,P., Spark, Streamer, Proportional and Drift Chambers, Richelieu, London, (1974):

71. Sauli,F., CERN Report 77-09, CERN, Geneva, Switzerland (May 1977).

72. Baum,H., et al., NIM 152, 541 (1979).

73. KTrsten,F.A., Ann.Rev.Nucl.Sc1. 25, 5i0 (1975) discusses CAMAC.

74. Mfg. EMI Corp., Plainview, NY 11803, USA.

75. Aret1,H., et al., NIM 212, 135 (19983). 


\section{$-204-$}

76. Barsotti,E., IEEE Trans.Nucl.Sc1. NS-26, 686 (1979).

77. Barsotti,E., et al., Unpublished TM-821 2nd ed., Fermilab, Batavia, IL 60510, USA. (1979).

78. Hammond,H.W., and M.S.Ewing, DECUS Program Library Routine 11-32, DECUS, Marlboro, MS 01752, USA (November 1980).

79. Mfg. Digital Equipment Corp., Maynard, MS 01754, USA:

80. Beili,J., and R.J.Dosen, Unpublished PN-112, Fermilab, Batavia, IL 60510, USA. (November 1979).

81. Beil, J., and R:J.Dosen, Unpublıshed PN-148, Fermilab, Batavia, IL 60510, USA. (May 1979).

82. Mf g. Digital Pathways, Mt.View, CA 94043, USA.

83. Akerlof,C. and Nitz,D., Proc. Digital Equipment Úsers'Society, Sań Diego Meeting, DECUS; Marlboro, MS 01752, USA (November 1980).

84. Akerlof, C., and $N_{1} t z, D .$, Unpublished SUPERGRAM Users Guide, University of Michigan, Ann Arbor, MN 48104, USA (1980).

85. Kraushsaar,P., Unpublıshed Thesis, University of Michigan, Ann Arbor MN 48104, USA., (November 1982).

86. Mfg. Control Data Corp., Minneapolis, MA 55440, USA.

87. Saqulı,F., CERN Report 77-0'9 pg.52, CERN, Geneva, Switzerland, (May 1977).

88. 'Koi zum1,G., Unpublished Technical Memo TM-786, Eermilab; Batavia, IL 60510, USA., (May 1978).

89. This program was written by R.Wagner of Fermilab.

90. For example see Roy,R., and Nigram,B., Nuclear Physics, Wiley, N.Y. NY, USA., for a discussion of the Fermi Gas Model.

91. Moniz,E., et ai., Phys.Rev.Lett. 26, 445 (1971).

92. The Ferm 1 momentum for $\mathrm{Cu}$ was taken to be the same as for $\mathrm{N} \perp$ as measured by $[91]$.

93. The Fermi momentum for Be was interpolated between $\mathrm{Li}$ and $\mathrm{C}$ [91] using $\mathrm{p}_{\text {Fermi }}=\mathrm{C}_{0}+\mathrm{C}_{1} / \mathrm{A}$.

94. Fermi motion effects are discussed by Bodek, A., and Ritchie,J.; Phys.Rev.D 23, 1070 (1981).

95. Anassontzis,E., et.al, Unpublished Preprint Fermilab-CONF 82/50-EXP, Fermilab, Gatavia, IL 60510, USA., (July 1982).

96. James, F., Rep.Prog.Phys $42,31145^{\circ}$ (1983). 


\section{$\cdot-205-$}

97. Common Library Mathematical" Routines, Pub. 60483100, Control Data Corp, Minneapol is MA 55440, USA.

98. NORRAN, CERN Program Library, Routine U101, CERN, Geneva, Switzerland.

99. Ahrens, J., and Dieter, U., Comm: ACM 15, 873.(1972).

100.Lindelof, T., CERN Program Library Routine V101, CERN, .Geneva, Switzerland.

101.Carroll,A.S., et al., Phys.Lett. 80B, 319 (1979).

102.R1chard-Serre,C., CERN Report 71-18, CERN, Geneva, Switzerland: (1971).

103.Serre,C., CERN Report 67-5, CERN, Geneva, Switzerland (1967). '

104.Joseph,P., NIM T5, 13 (1969).

105.Schrelder,Y.,ed., The Monte Carlo Method, Peragamon, London. (1966).

106 see for example, J.Badıer et al., Zeit.Phys.C 11, 195 (1981), or J.Ae Groot, Zeit.Phys.C 1, 143 (1979).

107.Eadie,W., et al., Statistical Methods"in Experimental Physics, Nor th Holland, Amsterdam, (1971).

108.Frodesen,A., et al., Probability and Statistics in Particle Physics, Universitetforlaget, Oslo (1979).

109.Johnson empirical distributions are discussed by Eadie et al., $[107]$.

110.James,F., and Roos,M., CERN Program Library Routıne D506, CERN, Geneva, Switzerland (1971):

111. MINUIT uses the Davidon variable matrix algorithm lescribed in Davidon,W. Computer J. 10, 406 (1968).

112.Van Ginnekin,A., Unpublished Technical Memo FN-272, Fermilab, Batavia, IL 60510, USA. (Jan. 1975).

113. Duke,D., and J.Owens, Unpubli'shed Preprint FSU-HEP-831115, Florida State University, Tallahasse, EA 32306, USA (1983).

114.Lipps,H., CERN Program Library Routine D112, CERN, Geneva, Switzerland (1979).

115.Aubert,J .,et al., Phys.Lett. 105B, 315 (1981)

116.Clark,A., et al., Phys.Rev.Lett. 51, 1826 (1983).

117.MacFarlane,D., et al., Unpublished Preprint Fermilab 83/108-EXP, Fermilab, Batavia, IL 60510, USA. (January 1984).

118.Abramowicz,H., et, al., Zelt.Phys.C 17, 283 (1983). 
119.Aubert,J., et al., Phys.Lett. 123B', 123 (1983).

120.Aubert,J., et al., Phys.Lett. 105B, 322 (1981).

121.Bodek,A., et al., Phys.Rev.D 20, 1471 (1979).

122.Devoto,A., et al., Phys.Rev.D 27, 508 (1983).

123.Barker,I., B.Martin and G.Shaw, Zeit.Phys.C 19, 147 (1983).

124.Brodsky,S., and Farrar,G., Phys.Rev.Lett. 31, 1153 (1973).

o 125.Brodsky,S., and Farrar,G., Phys.Rev.D 11, 1309 (1975).

126. Badier,J., Phys.Lett. 96B, 422 (1980).

127. Callot, O., Unpublished Thesis, Universite de Paris Sud, Orsay, France (1981).

128. Newman, C., Unpublished Thesis, University of Chicago, Chicago, IL 60637, USA. (March 1979).

129.Farrar,G., Nucl.Phys. B77, 429 (1974).

130. Bądier, J., et a1., Zèit.Phys.C 18, 281-287 (1983).

131.Ross1,B., and Greisen,K., Rev.Mod.Phys. _13, 240 (1941).

132.Rossi,B., High Energy Particles, Prentice Hall, Englewood Cliffs, NJ ,USA. (1952).

133.Scott,W.T., Phys.Rev. 76, 212 (1949).

134.Badier,J., et al., NIM 175, 319 (1980).

135.zXSSQ, IMSL Library Reference Manual, 9th ed., IMSL, Houston, TX, 77036, USA (1982). 